\title{
WestVirginiaUniversity
}

THE RESEARCH REPOSITORY @ WVU

Graduate Theses, Dissertations, and Problem Reports

2002

\section{Slow wave ion heating and parametric instabilities in the HELIX helicon source}

John L. Kline

West Virginia University

Follow this and additional works at: https://researchrepository.wvu.edu/etd

\section{Recommended Citation}

Kline, John L., "Slow wave ion heating and parametric instabilities in the HELIX helicon source" (2002). Graduate Theses, Dissertations, and Problem Reports. 1592.

https://researchrepository.wvu.edu/etd/1592

This Dissertation is protected by copyright and/or related rights. It has been brought to you by the The Research Repository @ WVU with permission from the rights-holder(s). You are free to use this Dissertation in any way that is permitted by the copyright and related rights legislation that applies to your use. For other uses you must obtain permission from the rights-holder(s) directly, unless additional rights are indicated by a Creative Commons license in the record and/ or on the work itself. This Dissertation has been accepted for inclusion in WVU Graduate Theses, Dissertations, and Problem Reports collection by an authorized administrator of The Research Repository @ WVU.

For more information, please contact researchrepository@mail.wvu.edu. 


\title{
Slow Wave Ion Heating and Parametric Instabilities in the HELIX Helicon Source
}

\author{
John L. Kline \\ Dissertation submitted to the College of Arts and Sciences \\ at West Virginia University \\ in partial fulfillment of the requirements \\ for the degree of \\ Doctor of Philosophy \\ in \\ Plasma Physics \\ Earl Scime, PhD., Chair \\ Fred L. King, PhD. \\ Larry E. Halliburton, $\mathrm{PhD}$. \\ John E. Littleton, PhD. \\ Timothy N. Good, PhD. \\ Department of Physics \\ Morgantown, West Virginia \\ 2002
}

Keywords: Helicon, Ion Heating, Slow wave, Trivelpieve-Gould, Lower Hybrid, Parametric Decay, Parametric Instabilities 


\section{ABSTRACT \\ Slow Wave Ion Heating and Parametric Instabilities in the HELIX Helicon Source}

\section{John L. Kline}

The primary focus of the experiments described here is to determine the mechanism responsible for intrinsic ion heating in helicon sources. Two possible mechanisms have been identified: ion Landau damping of the slow wave and parametrically driven instabilities. Consistent with ion Landau damping of the slow wave, the perpendicular ion temperatures $35 \mathrm{~cm}$ downstream of the RF antenna are largest when the RF frequency matches the local lower hybrid frequency; the condition at which the slow wave has a maximum in perpendicular wave number (perpendicular with respect to the applied magnetic field) due to a lower hybrid resonance. The ion temperatures also peak at the edge of the plasma where theory predicts the slow wave should have the largest amplitude and perpendicular wave number. Consistent with ion heating due to parametrically driven instabilities, parametrically driven low frequency waves are observed for the same conditions at which the ion temperatures $5 \mathrm{~cm}$ downstream of the $\mathrm{RF}$ antenna are largest. The measured characteristics of the low frequency wave suggest that the wave is an electrostatic ion acoustic wave. The electrostatic and electromagnetic features of the parametrically driven waves as a function of magnetic field and RF frequency are also presented and discussed. 
In loving memory of my grandmother:

\section{Edith Alberta Shirley Jones}

July 11, 1920 - February 26, 2000

May I have only a fraction of her determination. 


\section{Acknowledgements}

First, I want to thank my parents, Lee and Louise Kline, not just for my birth, but for all of their support over my long career as a student. To my dad I have two things to say, "Alligator mouth, Alligator ass" and "just think what I could do with some of those better genes." I want to thank my lovely wife, Alina, for all her love and support. I also want to thank my sisters, Pam and Carolyn, and my extended family. They have always been there for me. Thanks to my Aunt Kathryn for the home made jelly she has supplied while I have been here at WVU. Thanks also to my lifelong friends: David Semon, Dwight Herron, Scott Pisula, Craig Ruliffson, Marcus Berminge, George Forbes, and Dwayne White, on whom I could always count.

I am grateful I have had the opportunity to work with Professor Earl Scime. Not only has he helped direct my graduate research, but he has taught me an incredible amount, given me opportunities that I would not otherwise had, and been someone I count on professionally and personally. He has been a real friend.

I want to thank my colleagues, Dr. Paul Keiter, Dr Matt Balkey, and Dr. Robert Boivin, for many technical and scientific interactions. The guys in the machine shop: Tom, Doug, and Carl have been gracious with their time and patience. I appreciate that whenever I need something, they were always there to help; even when I needed it yesterday. I also want to thank Sherry and Siobhan for the help in the department office. I thank Xuan Sun and Amy Keesee for their assistance in taking some of the data presented in this dissertation. Finally, I would like to thank Professor R. P. Treat for his help and encouragement in my first year of graduate school.

Because of my non-traditional career path, many people have been influential in my life. Some of the most influential were my coworkers at WSSC. I owe a debt of gratitude to Jake Brodsky. He not only encouraged me to learn and go back to school, but inspired me with our technical and scientific conversations. I enjoyed riding with him in the Piper and I hope to follow his example in life. I also want to thank Ron Strawder for hiring me. With him as my boss, I was able to mature, grow, and find my direction in life. Both he and his boss, Frank Grimes, put me in a responsible position, encouraged me, and let me grow and excel. There were also the engineers that 
encouraged and taught me: Jim Kraus, Gary Grey, Mike Martin, and Phil Barlow. I thank Cathy Hilton-Russo for editing my essays in my literature class, and the other friends that worked at WSSC, Ron Mason, Charlie Rupprecht, Doug Barlow, and George Shambley.

Many other people have helped me get to this point in my life. Since listing all of them would fill another dissertation, I will stop here and get on with the show!

Thank you all,

John Lee Kline

Morgantown, WV

March 15, 2002 


\section{The Road Not Taken}

TWO roads diverged in a yellow wood, And sorry I could not travel both And be one traveler, long I stood And looked down one as far as I could To where it bent in the undergrowth; Then took the other, as just as fair, And having perhaps the better claim, Because it was grassy and wanted wear; Though as for that, the passing there Had worn them really about the same, And both that morning equally lay In leaves no step had trodden black. Oh, I kept the first for another day! Yet knowing how way leads to way, I doubted if I should ever come back. I shall be telling this with a sigh Somewhere ages and ages hence:

Two roads diverged in a wood, and II took the one less traveled by, And that has made all the difference.

-- Robert Frost 


\section{Table of Contents}

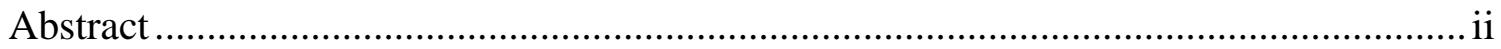

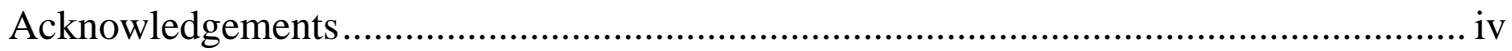

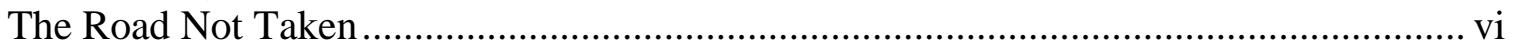

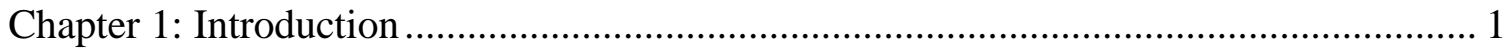

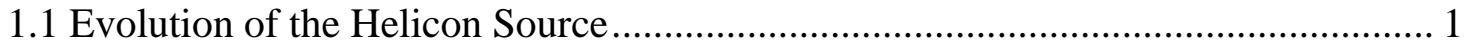

1.2 The Development of the HELIX Helicon Source ................................................. 5

1.3 Active Areas of Helicon Source Research....................................................... 18

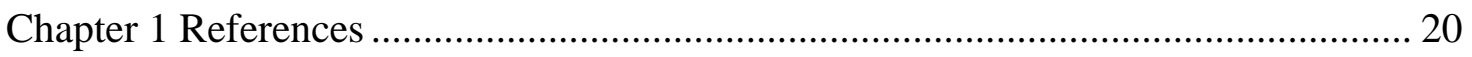

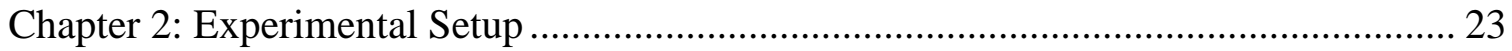

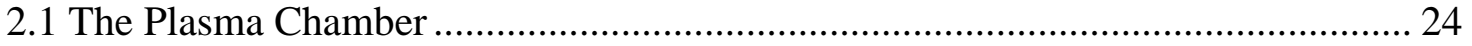

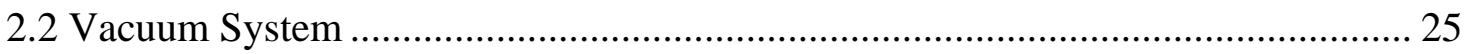

2.3 The Magnetic field ........................................................................................... 25

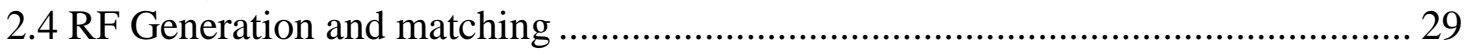

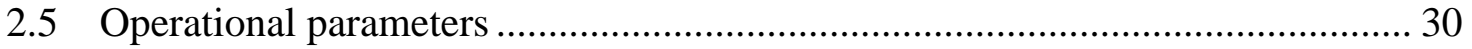

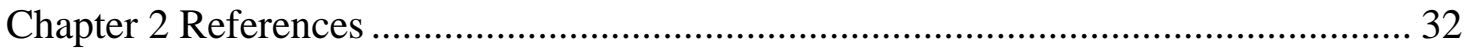

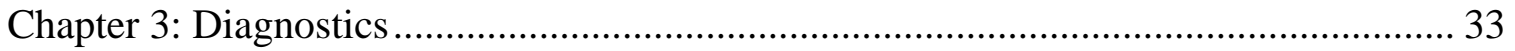

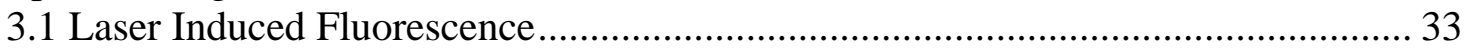

3.1.1 Laser Induced Fluorescence of Argon Ions ............................................ 34

3.1.2 Laser Induced Fluorescence Apparatus .................................................. 37

3.1.3 Laser Induced Fluorescence Data Analysis ................................................ 43

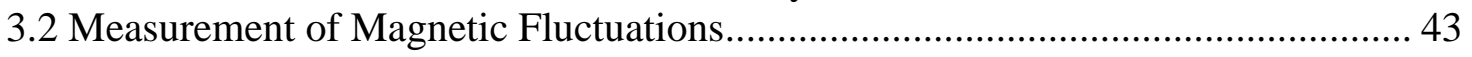

3.2.1 Magnetic Fluctuation Coil Theory ........................................................ 44

3.2.2 Magnetic Fluctuation Coil Apparatus ..................................................... 45

3.2.3 Wave Number Analysis for Fixed Probe Pairs:
Magnetic Fluctuation Coils and Electrostatic Probe $. . . \ldots \ldots \ldots . . . \ldots . . . . . . . . . .50$

3.3 Measurements of Electron Temperature and Plasma Density (Langmuir Probe) .. 58

3.3.1 Langmuir Probe Theory ........................................................................ 58

3.3.1.1 Determination of Electron Density and Temperature from

Langmuir Probe Data .......................................................................... 59

3.3.1.2 Magnetic Field Effects on Langmuir Probes .......................................... 61

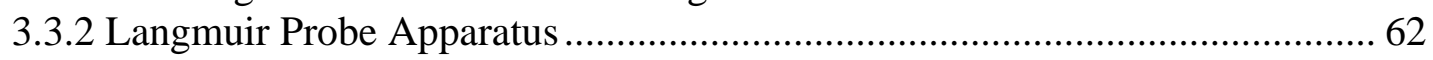

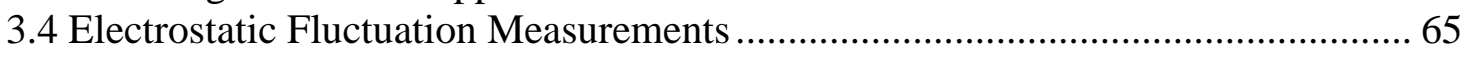

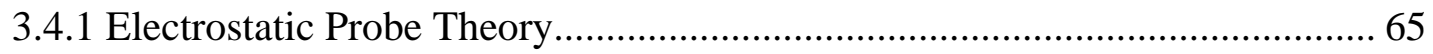

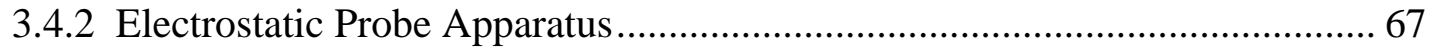

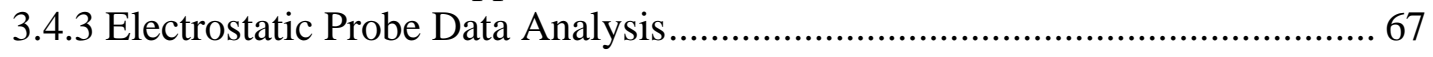

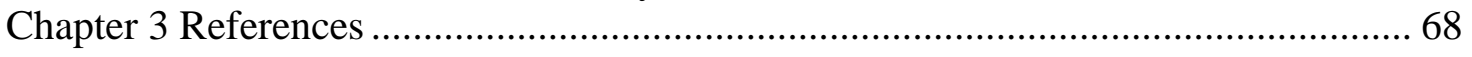

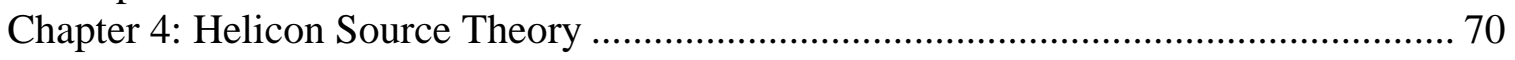

4.1 Cold Plasma Theory for Helicon Sources........................................................ 75

4.2 Cold Plasma Computational Model for Helicon Sources ................................... 78

4.2.1.1 Plasma Density Effects on Solutions to the Cold Plasma Dispersion

Relation ..................................................................................... 79

4.2.1.2 Parallel Wave Number Effects on Solutions to the Cold Plasma

Dispersion Relation.......................................................................... 83 
4.2.1.3 Wave Frequency Effects on Solutions to the Cold Plasma Dispersion

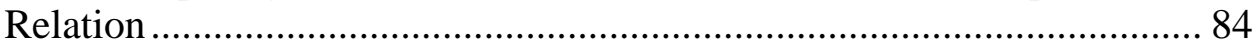

4.2.2 The Cold Plasma Computational Model for Arbitrary Density Profiles ......... 85

4.2.3 Maximum Values of $k_{\perp}$ for the Slow Wave as a Function of RF Frequency and Magnetic Field Strength ................................................ 88

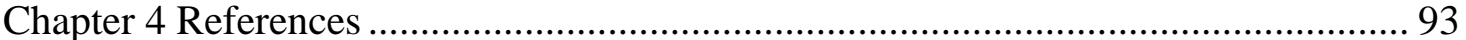

Chapter 5: Ion Temperature Measurements................................................................. 97

5.1 The Ten Electromagnet Configuration .............................................................. 97

5.2 The Eight Electromagnet Configuration ............................................................... 104

5.3 Implications of Ion Temperature Measurements ................................................... 109

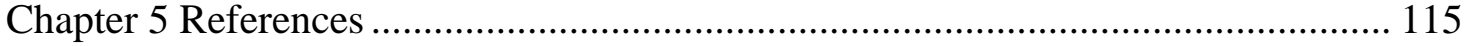

Chapter 6: Parametric Instabilities and Coupled Modes.................................................. 117

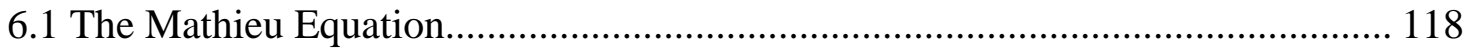

6.2 Parametric Coupling and Instabilities in Plasmas.............................................. 122

6.3 Other Parametrically Driven Instabilities ……................................................ 127

Chapter 6 References ............................................................................................ 131

Chapter 7: Electrostatic and Electromagnetic Fluctuation Measurements ..................... 133

7.1 Evidence of Parametrically Driven Electrostatic Waves ...................................... 134

7.2 Characteristics of the Parametrically Coupled Electrostatic Waves in HELIX.... 136

7.2.1 Variation of Parametrically Coupled Electrostatic Waves with RF Power ... 136

7.2.2 Variation of Parametrically Coupled Electrostatic Waves with Radius ........ 138

7.2.3 Variation of Parametrically Coupled Electrostatic Waves with

RF Frequency and Magnetic Field Strength ................................................ 140

7.2.4 Wave Number Measurements for the Parametrically Coupled

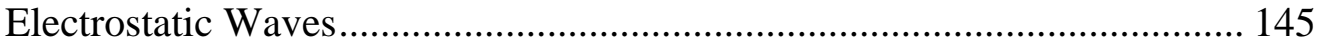

7.3 Characteristics of Electromagnetic Waves in HELIX ........................................ 149

7.3.1 Wave Number Measurements for the Primary Electromagnetic Wave......... 149

7.3.2 Electromagnetic Fluctuation Spectra ............................................................. 152

7.4 Implications of the Electrostatic and Electromagnetic Fluctuation

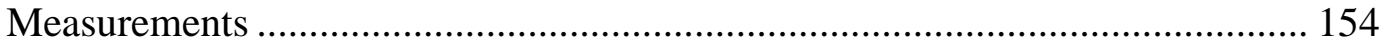

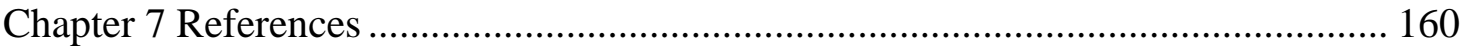

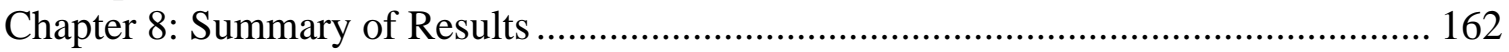

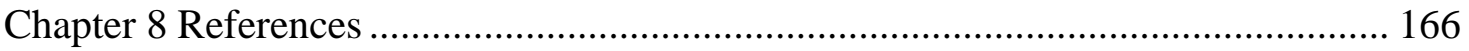

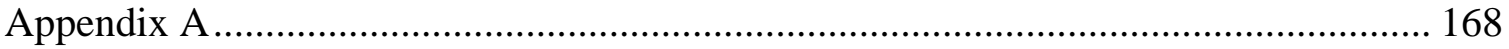

Vitae 


\section{Chapter 1: Introduction}

\subsection{Evolution of the Helicon Source}

The term Helicon was coined by Aigrain [Aigrain, 1960] in 1960 to describe the propagation of bounded right hand circularly polarized waves or whistler waves in a solid rod of sodium. These waves obeyed the characteristic dispersion relationship for whistler waves propagating along the magnetic field at frequencies between the ion and electron cyclotron frequencies $\left(\omega_{\mathrm{ci}}<<\omega<<\omega_{\mathrm{ce}}\right)$

$$
N^{2} \approx \frac{\omega_{p e}^{2}}{\omega \omega_{c e} \cos \theta} .
$$

where $N$ is the parallel index of refraction $\left(N=k_{\|} c / \omega\right), c$ is the speed of light, $k_{\|}$is the wave number parallel to the magnetic field, $\omega_{p e}$ is the electron plasma frequency, $\omega_{c e}$ is the electron cyclotron frequency, and $\theta$ is the angle with which the wave propagates with respect to the ambient magnetic field. In the mid 1960's, Lehane and Thonemann [1965] performed the first experiments involving bounded whistler or "helicon" waves in a laboratory plasma. Although they used a radio frequency, RF, plasma source, a separate antenna was used to launch the whistler waves. There is some speculation, however, that their plasma source RF antenna may have been at least partly responsible for the observed helicon waves [Boswell and Chen, 1997]. A few years later, Boswell [Boswell, 1970] used a single antenna both to generate the plasma and to launch bounded whistler, helicon, waves. Boswell's plasma source is considered the first helicon source. Even though the helicon source produces highly ionized (> 80\%), high-density $\left(n \sim 1 \times 10^{13}\right.$ $\mathrm{cm}^{-3}$ ) plasmas, very little effort has been devoted to understanding the helicon source itself. In later years, Boswell continued to study whistler wave propagation in RF generated magnetoplasmas, but launched the whistler waves using a secondary antenna [Boswell, 1975b; Boswell, 1975a].

In the mid 1980's, demand for integrated circuits exploded and the need for new high-density plasmas sources for plasma processing of semiconductors sparked new interest in the helicon source. The helicon source was believed to be a good candidate for 
etching smaller-scale features to increase the number of electronic components on a single silicon wafer [Perry et al., 1991; Boswell and Chen, 1997; Chen and Boswell, 1997; Balkey, 2000]. The primary scientific efforts focused on explaining the high ionization efficiency of the helicon source and how to optimize the helicon source for plasma processing. These studies involved characterizing plasma parameters of the source and measuring the wave fields of the helicon wave as a function of magnetic field strength, neutral pressure, RF power, and driving frequency [Chen, 1991; Chen, 1992; Chen and Decker, 1992; Chevalier and Chen, 1993; Shoji et al., 1993; Chen et al., 1997].

In the 1990's, the types of experiments using helicon sources began to change. The first application of a helicon source in a non-plasma processing experiment was as a plasma source for the HELAC-1 stellarator [Loewenhardt et al., 1991]. In 1995, the Hot hELicon eXperiment (HELIX) was built at West Virginia University (WVU) as a plasma source for high beta (particle pressure/magnetic field pressure, i.e., $\beta=n k_{b} T \mu_{o} / B^{2}$ ) magnetospherically relevant plasma physics [Keiter et al., 1997]. RF plasma sources had been previously used to study magnetoplasmas [Boswell, 1975a], but not by using a helicon source to produce highly ionized, high beta plasmas. The high beta plasmas are created by letting the high-density plasma produced by HELIX flow into a large diffusion chamber embedded in a weak magnetic field, the Large Experiment on Instabilities and Anisotropies (LEIA).

In the past three years, helicon sources have become more popular for basic plasma science experiments. Recently, Watts [Hanna and Watts, 2001] and Klinger [Franck and Klinger, 2000] have built helicon sources to study Alfvén waves as well as other wave phenomena. The helicon plasma source is an ideal choice for their experiments. Since the helicon source produces high plasma densities, the Alfvén wavelength in their experiments is short enough to fit in plasma chambers 1 - 2 meters long. At wave frequencies of half the ion cyclotron frequency, the Alfvén wavelength scales as the reciprocal of the square root of density, $\lambda \sim 1 / \sqrt{ } n$. Not only are helicon sources being used for Alfvén wave experiments, helicon sources are also being considered as possible plasma propulsion sources for deep space missions, e.g., VASMIR [Chang Diaz et al., 1999] and MNX [Cohen et al., 1999]. Helicon sources have also been built in toroidal geometries to study fusion relevant instabilities and coherent modes [Grulke et al., 2001]. 
A torodial helicon source has been proposed at WVU to study low phase velocity current drive. These experiments may add to the body of fusion knowledge at a fraction of the cost of large-scale tokamak experiments, e.g., DIII-D and Alcator C-mod. Because of the high-density nature of the helicon source, fusion relevant science can be performed as long as the effects of much lower plasmas temperatures are taken into account. For example, Light et al. [2001] studied low frequency $(2 \mathrm{kHz})$ electrostatic fluctuations that occur at high magnetic field strengths ( $>800$ Gauss) in helicon sources. These low frequency instabilities could be responsible for anomalous plasma diffusion that reduces the plasma density in helicon sources at high magnetic fields. Light's investigation of turbulent diffusion is just one example of how helicon source experiments can address fusion relevant science questions.

The new role of the helicon source has resulted in the discovery of new phenomena in helicon sources. Using laser induced fluorescence (LIF), Scime et al. [1998] measured ion temperatures in a helicon source for different plasma parameters. Those measurements demonstrated that the ions in helicon sources were not cold, as had been assumed, but had substantial temperatures. The measurements showed that the perpendicular ion temperature increased with magnetic field and could be anywhere from $0.1 \mathrm{eV}$ to $1 \mathrm{eV}$. Although the perpendicular ion temperature increased with magnetic field, the parallel ion temperature remained nearly constant. Balkey et al. [2001] completed a comprehensive study of perpendicular ion temperature, electron density, and electron temperature as a function of magnetic field and driving frequency for multiple types of antenna geometries. That study showed that the perpendicular ion temperature was a maximum when the RF driving frequency was below the on-axis lower hybrid frequency, $\omega_{l h} \cong\left(\omega_{c e} \omega_{c i}\right)^{1 / 2}$, and the plasma density was a maximum when the RF frequency equaled the on-axis lower hybrid frequency.

Why are these discoveries important? When the helicon source was only used in plasma processing applications, the most important issues in helicon source research were decreasing the electron temperature and increasing electron density. The discovery of finite ion temperatures has important implications for plasma processing. Finite ion temperatures limit the size of the structures that can be etched. In addition, for the helicon source to be used for basic plasma science experiments, a complete 
understanding of how the source works is critical. Only with a complete understanding of helicon source physics can decisions can be made as to what basic science experiments are appropriate. Sometimes, a more complete understanding of helicon sources can make certain basic science experiments easier. In the case of the high beta temperature anisotropy experiments, the inherent ion temperature anisotropy in helicon source plasmas eliminated the need to create the ion temperature anisotropy externally. Intrinsic ion temperature anisotropy can also affect the design of plasma propulsion experiments employing helicon sources. In the VASIMR experiment, ion cyclotron resonant heating will be used in increase the perpendicular ion temperature [Chang Diaz et al., 1999]. Thrust is obtained by converting the perpendicular temperature into parallel momentum through conservation of magnetic moment in a magnetic nozzle field at the end of the helicon source. An understanding of how to operate the helicon source to maximize the perpendicular ion temperatures is clearly important in such experiments.

Another example of the need for better understanding of helicon source physics involves Keiter's observations of electromagnetic waves in the $8-10 \mathrm{kHz}$ range in LEIA. The measured wave amplitudes scaled with the magnetic field in HELIX [Keiter, 1999; Scime et al., 2000]. The current hypothesis is that the waves were either resistive drift waves or instabilities driven by velocity shear in the helicon source. Measurements of the parallel flow indicate that parallel velocity shear does in fact exist in HELIX [Sun et al., 2001]. At this time, there is no conclusive evidence that the observed waves were driven by parallel velocity shear. However, the very existence of parallel velocity shear suggests that experiments concerning shear modified instabilities can also be performed in helicon sources.

The original topic of this dissertation was "A Space-Relevant Study of Electron Heat Flux Instabilities." However, it proved more difficult than expected to produce a twoelectron component plasma in LEIA with a relative drift between components greater than the Alfvén speed. Plasmas with high enough beta to reduce the Alfvén speed $\left(\mathrm{v}_{a} \sim\right.$ $1 / \sqrt{ } \beta$ ) could not be produced without reducing the relative velocity of the two components. A second dissertation topic considered was a continuation of Keiter's [1999] work on ion temperature anisotropy driven instabilities. However, after the replacement of the cracked all-glass vacuum chamber with a hybrid stainless steel-glass 
vacuum chamber, high perpendicular ion temperatures could no longer be obtained in HELIX [Scime et al., 1998]. Further investigation suggested that the loss of ion temperature anisotropy was due to changes in the magnetic field geometry. During installation of the stainless steel chamber, the antenna was moved to a region of the experiment with a larger magnetic field gradient and overall lower magnetic field strength. It was hypothesized that the lower magnetic field strength in the antenna region somehow eliminated the poorly understood ion heating mechanism. After the magnetic field was made uniform throughout the antenna region, the ion heating reappeared. A desire to better understand the ion heating mechanism in helicon sources motivated the experiments described in this dissertation.

\subsection{The Development of the HELIX Helicon Source}

The first helicon source at WVU, HELIXa, was completed in April of 1996. HELIXa was built as a plasma source for magnetospherically relevant laboratory plasma experiments. Even though space-relevant plasma experiments are the focus of the WVU research group, considerable time has been spent understanding helicon plasmas.

HELIXa was free standing and was never connected to the Large Experiment on Instabilities and Anisotropies (LEIA). Upon completion of HELIXa, experiments were performed to characterize the plasma source. Since HELIXa's plasma chamber was a single, $15 \mathrm{~cm}$ diameter, $157 \mathrm{~cm}$ long Pyrex tube with no port access along the sides, the first measurements were done from the end of the source with a RF compensated Langmuir probe [Sudit and Chen, 1994]. Due to the need to control the density for the planed experiments in LEIA, a variable RF frequency amplifier, $0.3-30 \mathrm{MHz}$, was used for plasma generation. According to the helicon dispersion relationship (Equation (1.1)), the density should have a $1 / f$ frequency dependence assuming the parallel wave number is constant. Rewriting the dispersion relationship in Equation (1.1),

$$
n \approx \frac{k_{\|}^{2} c B}{8 \pi^{2} e f} \cos \theta,
$$

where $n$ is the density, $B$ is the magnetic field strength, $e$ is the electron charge, and $f$ is the RF driving frequency. The dependence of density on the RF driving frequency had never been confirmed until the experiments of Keiter et al. [1997] at WVU and Kwak et 
al. [1997] at in Korea. Figure 1 shows the $1 / f$ density dependence reported by Keiter et al. [1997].

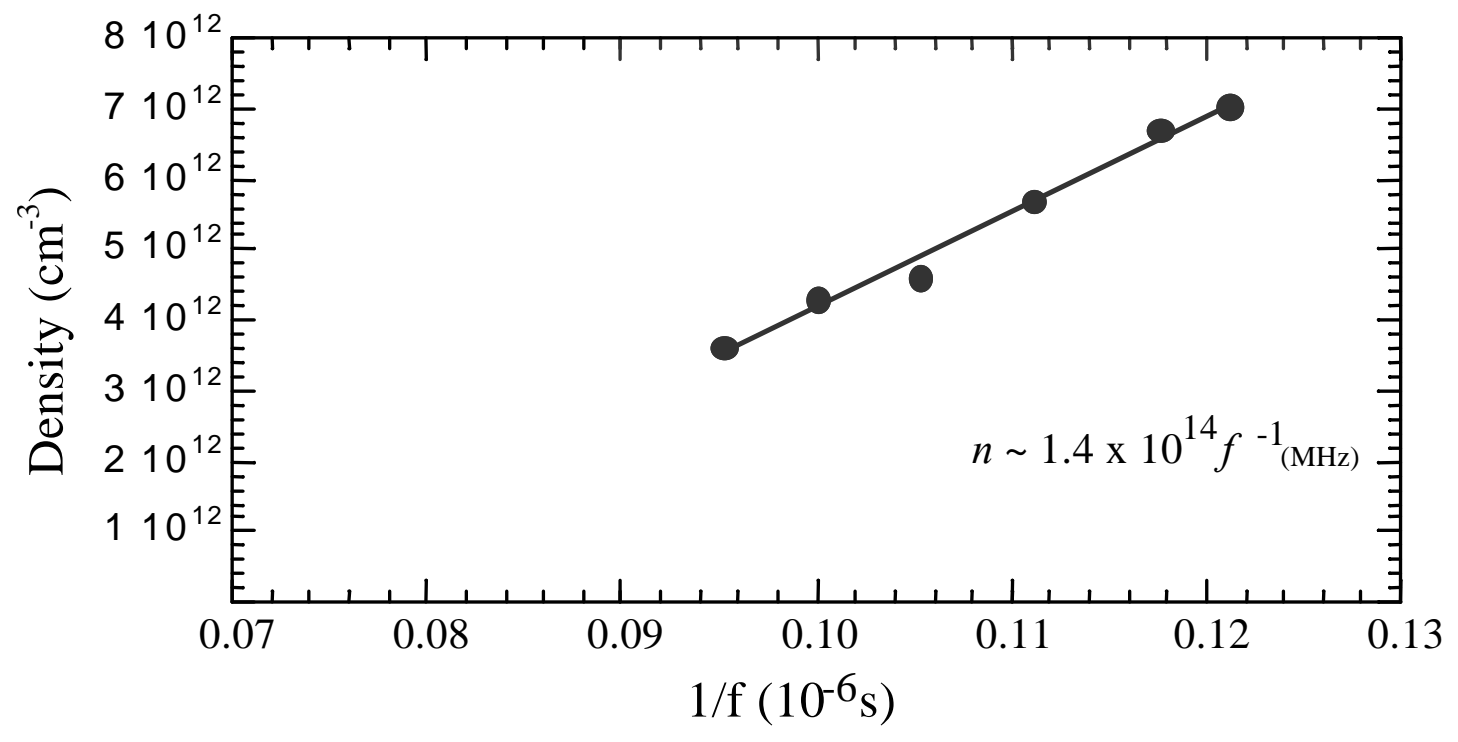

Figure 1: The 1/f density dependence observed in the HELIXa helicon source [Keiter et al., 1997].

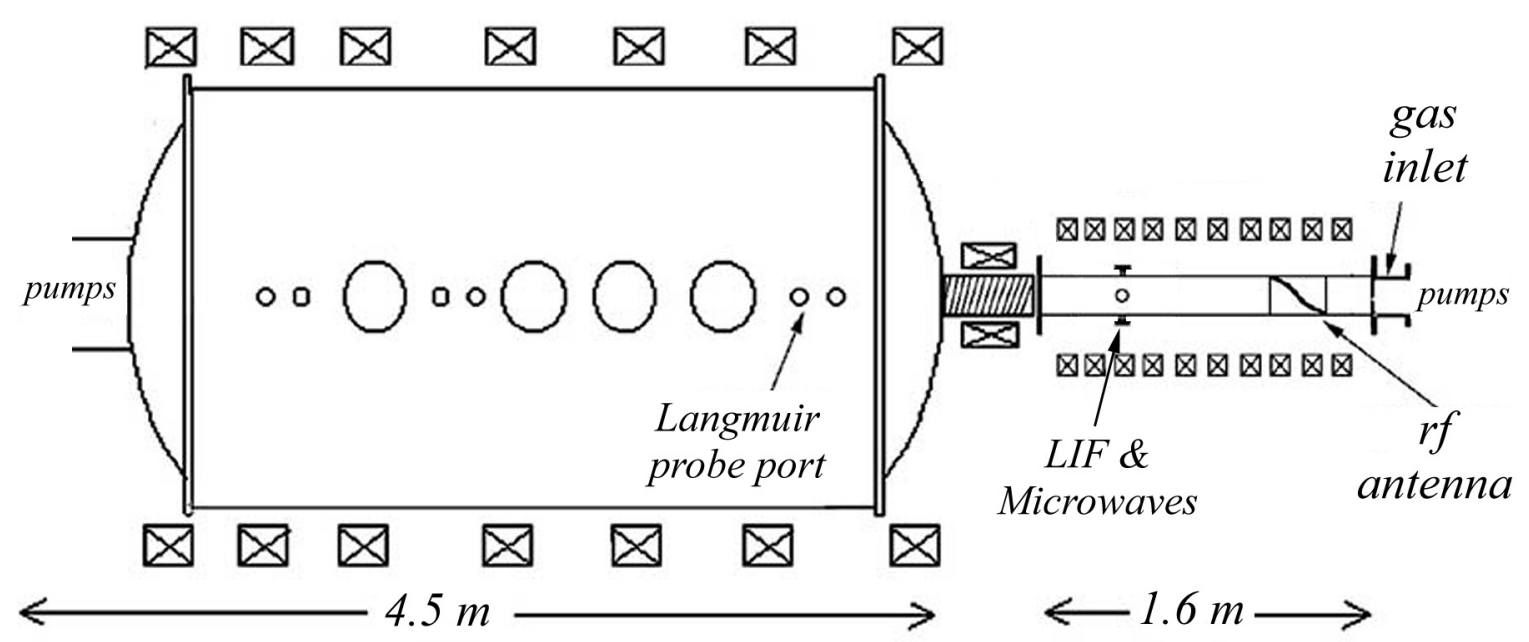

Figure 2: Schematic diagram of HELIX (HELIXb) and LEIA.

Another key issue for the planned experiments in LEIA was the ion temperature in HELIX. Anisotropic ion temperatures were necessary for the studies of space relevant, ion temperature anisotropy limited, high-beta space plasmas planned for LEIA. The ion temperature was measured using laser induced fluorescence [Stern and Johnson, 1975; 
Hill et al., 1983]. To provide access for the injected laser beam and the collection optics, a new plasma chamber was installed, HELIXb, and joined to LEIA (Figure 2). The new plasma chamber was $17 \mathrm{~cm}$ in diameter, $157 \mathrm{~cm}$ long, and included a set of four $23 \% 4$ Conflat $^{\mathrm{TM}}$ ports joined to the main chamber through 1" diameter Pyrex tubes. Using LIF in HELIXb, the first comprehensive ion temperature measurements in a helicon source were performed [Scime et al., 1998]. Although ion velocity distribution functions in helicon sources had been previously measured using LIF [Nakano et al., 1992], only two measurements were reported. They were both in the antenna region and each was for a different fill gas. In HELIXb, the ion temperatures in the perpendicular and parallel directions with respect to the ambient magnetic field were found to be anisotropic (Figure 3) and the anisotropy scaled with magnetic field strength. Even after these measurements were reported, other researchers continued to assume the ions in helicon sources were cold; on the order of room temperature. Concurrent with the LIF measurements at WVU, an auxiliary RF ion heating system was developed to heat the ions anisotropically. Even though auxiliary ion heating was successfully implemented [Kline et al., 1999], it was not required for the high beta, $T_{\perp} / T_{\|}>1$ experiments in LEIA.

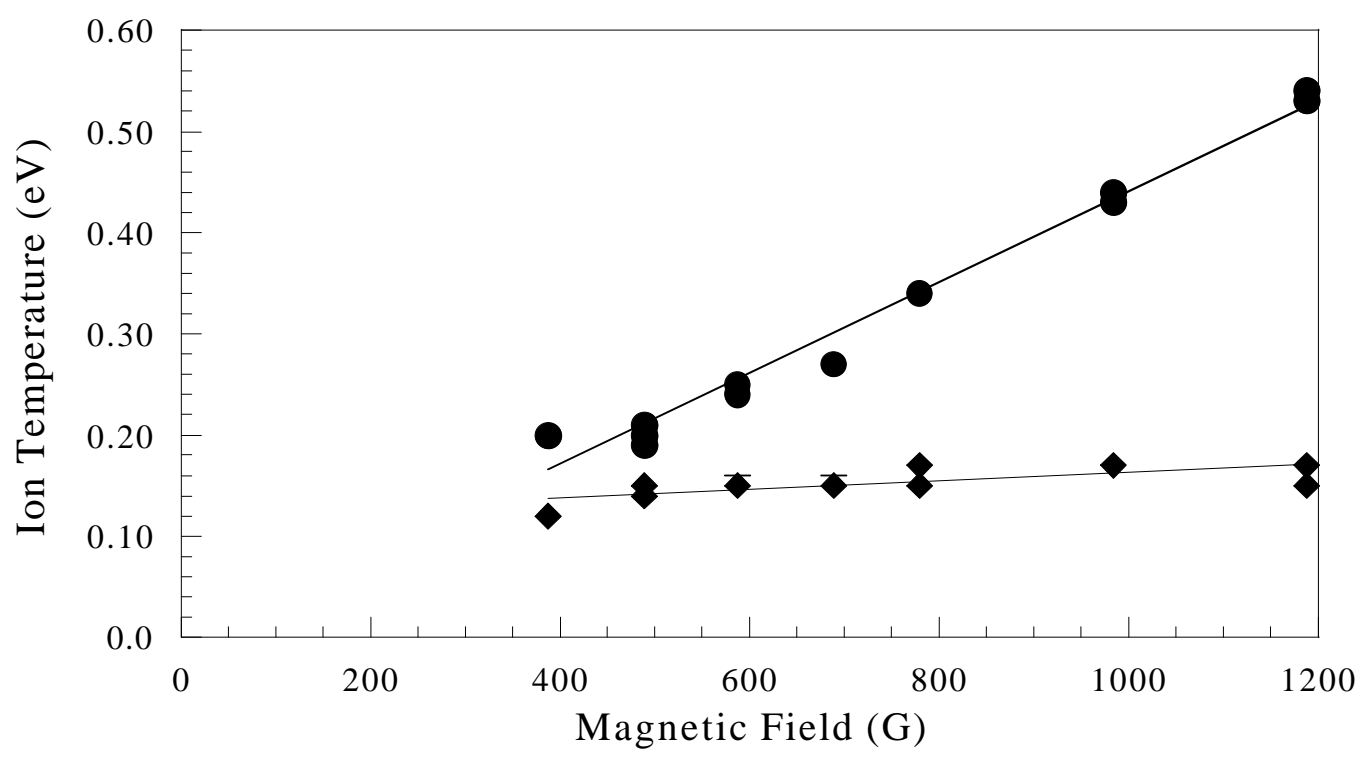

Figure 3: $(\bullet)$ Perpendicular and $(\bullet)$ parallel ion temperature measurements in HELIXb versus magnetic field strength for 400 Watt of RF power, 2 mTorr of neutral pressure, and a RF driving frequency of $9 \mathrm{MHz}$ [Scime et al., 1998].

Except for a few measurements by Nakano et al. [1992] and those reported by the WVU helicon group [Scime et al., 1998; Kline et al., 1999; Balkey et al., 2001], there are 
no other published LIF measurements of ion temperature in a helicon source. Recent measurements using a tunable diode laser LIF system developed at WVU also indicate ion temperatures greater than room temperature in the MNX helicon source [Boivin et al., 2002]. Both parallel ion temperature measurements and ion temperature measurements as a function of magnetic field strength remain to be accomplished in MNX.

In the fall of 1997, LEIA was completed and the two vacuum vessels, HELIXb and LEIA (Figure 2), were joined together. In 1999, the first space relevant, high beta experiments in LEIA were completed. The measurements clearly demonstrated a limit on the ion temperature anisotropy in high beta plasmas [Keiter, 1999] and the experimental results were consistent with theoretical predictions [Gary et al., 1994a; Gary et al., 1994b; McKean et al., 1994]. Wave activity consistent with excitation of Alfvén ion cyclotron instabilities was also observed [Scime et al., 2000]. However, ion neutral collisions were found to play an important role in limiting the observable range of ion temperature anisotropy in LEIA.

The issue of the ion neutral collisions highlights a characteristic of helicon sources that can create difficulties for basic plasma physics experiments employing helicon sources. The high densities, moderate neutral pressures, and low ion and electron temperatures in helicon sources make ion-neutral, ion-ion, electron-neutral, and electronelectron collisions problematic for experiments involving ions or low frequency waves. It is important to note, however, that even though the ion-ion collision frequency can be hundreds of times higher than the ion gyro frequency, low frequency, ion cyclotron waves can be excited in helicon sources [Kline et al., 1999]. The most likely explanation is that during collisions between like particles, e.g., ion-ion collisions, the particles merely exchange identity and momentum is conserved. Therefore, waves requiring coherent ion motion can still be excited in such highly collisional plasma.

After the completion of the first set of space related experiments in LEIA, the source of the ion heating in HELIX was still unknown. A subsequent comprehensive study of ion temperature, electron temperature, and electron density in a helicon source as a function of antenna geometry, RF frequency, RF power, neutral pressure, and magnetic field strength strongly suggested that the lower hybrid resonance plays an important role in ion heating and electron density production in helicon sources [Balkey, 2000]. The 
vacuum chamber geometry of HELIXb limited the measurements of the ion temperature to a single axial and radial location. An inability to insert probes into HELIXb plasmas also prevented the measurement of key plasma parameters in the source itself. To enable greater diagnostic access, a new hybrid vacuum chamber was designed and installed.

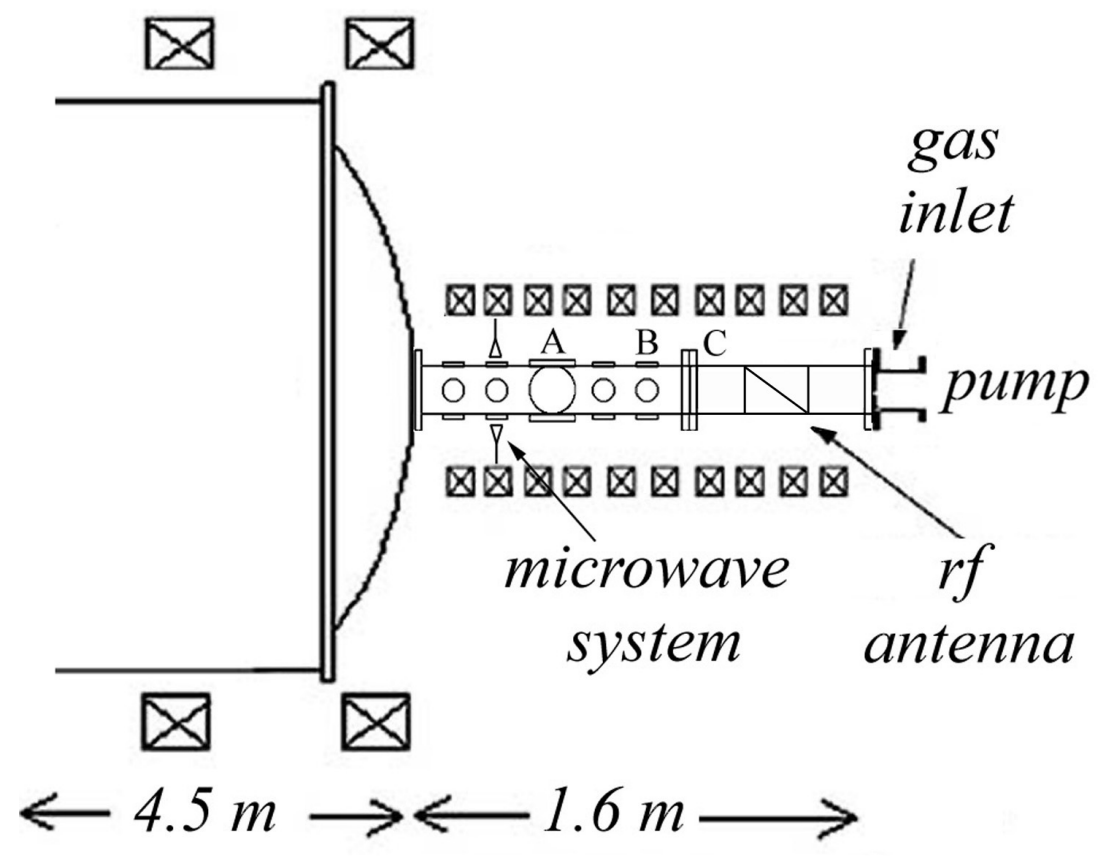

Figure 4: Schematic diagram of the hybrid stainless steel-glass chamber with a 6" diameter Pyrex section: HELIXc.

The new vacuum chamber, HELIXc (Figure 4), was part stainless steel and part Pyrex. The hybrid chamber consisted of a 24" long, 6" diameter Pyrex section connected to a 36 " long 6" diameter stainless steel section. The stainless steel section has four sets of four $23 / 4$ crossing ports with two sets evenly spaced on both sides of a set of four 6" Conflat ${ }^{\mathrm{TM}}$ crossing ports. When the new vacuum chamber was installed, the 6" bellows that connected HELIXb to LEIA was removed and the new vacuum chamber connected directly to the space chamber. The bellows was removed to increase the plasma density in LEIA by eliminating a possible region of enhanced plasma-wall interactions.

Measurements of the ion temperature in the new chamber, HELIXc, showed that the ions were relatively cold, $T_{i} \sim 0.12 \mathrm{eV}$, for any magnetic field strength. At the same parameters, typical ion temperatures in HELIXb were between $0.2 \mathrm{eV}$ and $1 \mathrm{eV}$. The 
densities measured in LEIA were also lower than expected and the plasma was visually unstable at high magnetic field strengths. It appeared that the boundary conditions of the new stainless steel chamber eliminated the mechanism that heated the ions and decreased density production. In an attempt to recover high ion temperature operation, an annular MACOR limiter was built to create a vacuum layer between the plasma and the stainless steel chamber wall. The inner diameter of the annular limiter was 4". The limiter, shown in Figure 5, was placed between the Pyrex and the stainless steel sections of the vacuum chamber. The limiter produced a 1" gap between the plasma edge and the stainless steel chamber. The ion temperatures with the limiter in place were even lower than before, $T_{i} \sim 0.08 \mathrm{eV}$. The limiter experiments suggested that the ion heating occurred at the edge of the plasma and by scraping off the plasma edge, the ion heating was eliminated.
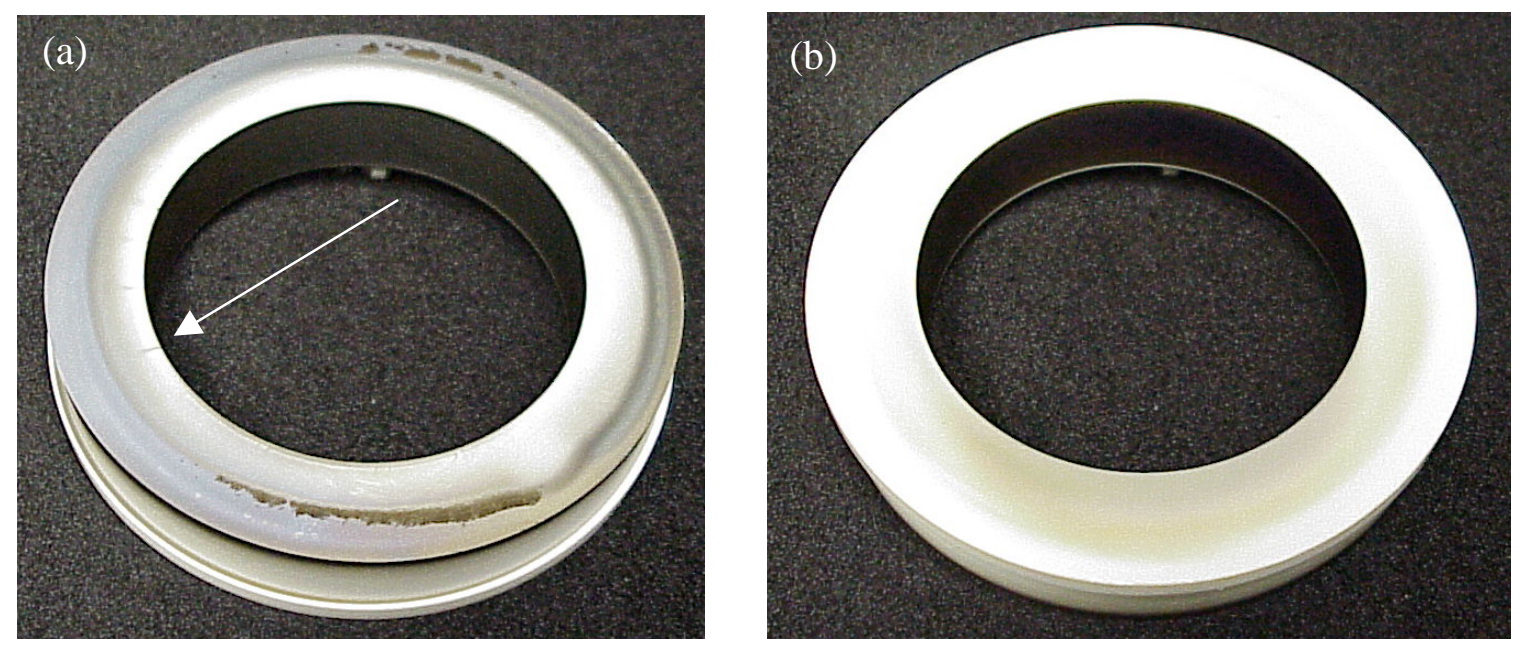

Figure 5: a) LEIA side and b) HELIXc side of the MACOR limiter used in HELIXc.

Installation of the limiter also affected helicon source performance. With the limiter in place, it was only possible to get a "blue core" plasma at high magnetic field strengths or high gas pressures. One possible explanation for this effect is that the limiter prevents surface waves, i.e., the "Trivelpiece-Gould" mode, from propagating from the Pyrex section into the stainless steel section except at high magnetic fields when the surface modes penetrate further into the plasma [Chen and Arnush, 1997]. 


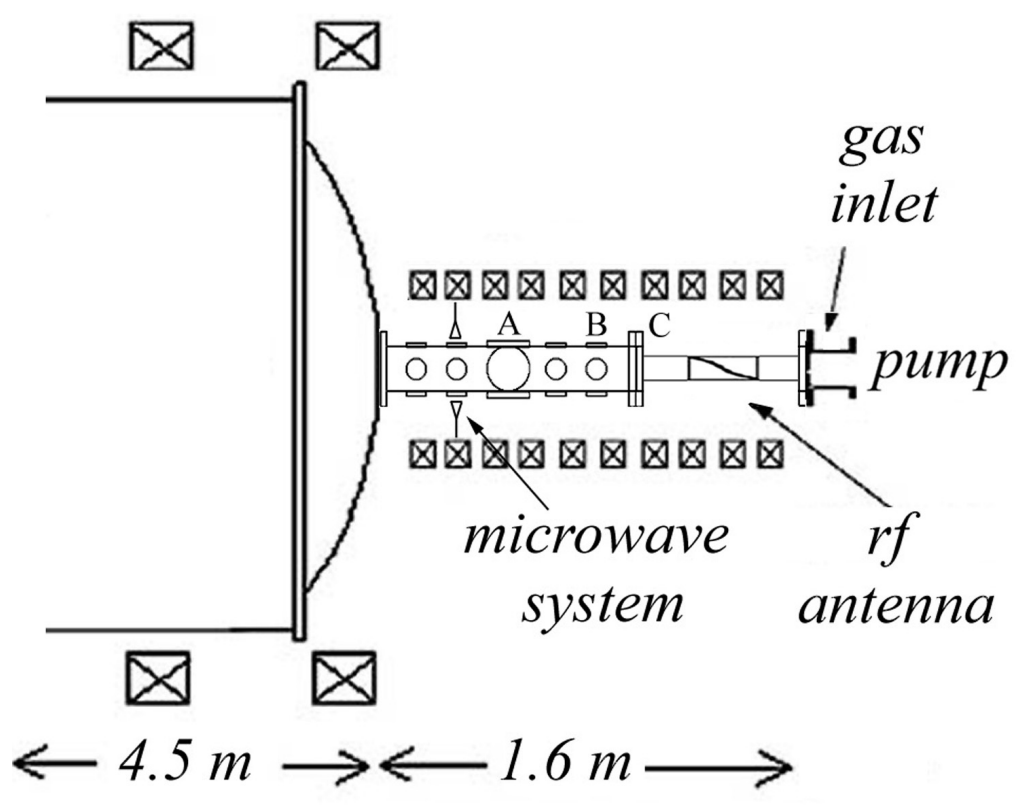

Figure 6: Schematic diagram of HELIX with a 4" diameter Pyrex section: HELIXd.

Since other helicon sources successfully using hybrid glass-metal vacuum chambers typically use glass sections with much smaller diameters than the metal chamber (as in the original HELIXb-LEIA configuration), the 6" diameter Pyrex tube was replaced with a 4" diameter Pyrex tube (HELIXd). The HELIXd configuration (Figure 6) has the same boundary conditions in the antenna region as HELIXc, but the edge plasma is not prevented from entering the metal portion of the vacuum chamber. Waves propagating in the plasma edge are also able to propagate into the stainless steel section. Initially, ion temperatures in HELIXd were low, $T_{i} \sim 0.13 \mathrm{eV}$, comparable to ion temperatures in HELIXc without the limiter.

During the installation of the HELIXc chamber, the RF antenna was moved away from the stainless steel chamber to prevent the possibility of the RF antenna arcing to the chamber and to limit inductive heating of the stainless steel chamber by the RF fields. Traditionally, the current in the first electromagnet in HELIX (on the far right in Figure 6) was reversed to create a strong magnetic field gradient on the backside of the antenna. The magnetic field at the new location of the RF antenna decreased sharply, creating a divergent magnetic field in the antenna region. Previous experiments had demonstrated that a magnetic field minimum behind the antenna can increase the plasma 
density in a helicon source [Chevalier and Chen, 1993], but in HELIXc the RF antenna was inside the field minimum region. The direction of the current in the first electromagnet was reversed to create a more uniform magnetic field profile and increased ion temperatures, $T_{i} \sim 0.5 \mathrm{eV}$, and increased plasma densities were recovered. Clearly, density production and ion heating were directly affected by the strength of the magnetic field in the antenna region. How a magnetic field gradient affects the density production and ion heating is not understood at this time. A set of experiments with varying levels of magnetic field gradient in the antenna region could provide additional information about both processes in helicon sources. In the experiments described in this dissertation, only the overall magnitude of a uniform magnetic field was varied.

Before delving into the details of the diagnostics, theory, and experimental observations relevant to the primary focus of this dissertation, a number of other interesting experimental observations are worth documenting. The helical $m=+1$ antennas used on HELIXc and HELIXd became asymmetrically (along the source axis) discolored after a few days of operation (Figure 7). The HELIX pumping station side (right side of antenna in Figure 6) of the RF antenna got considerably hotter than the other side. Other helicon groups using balanced matching circuits (balanced matching circuits will be discussed in Chapter 2), report similar observations [Franck, 2001]. Since the antenna is Ohmically heated by recirculating currents, the ac currents appear to be larger at one end of the antenna. On HELIXd, a $8 \mathrm{~cm}$ long helical $m=+1$ antenna was originally installed because previous results suggested shorter antennas would increase ion heating and plasma density [Balkey, 2000]. The shorter RF antenna was discolored in the same way as the $19 \mathrm{~cm}$ helical $m=+1$ antenna even though only low density, capacitive mode plasma could be generated with the shorter antenna. 


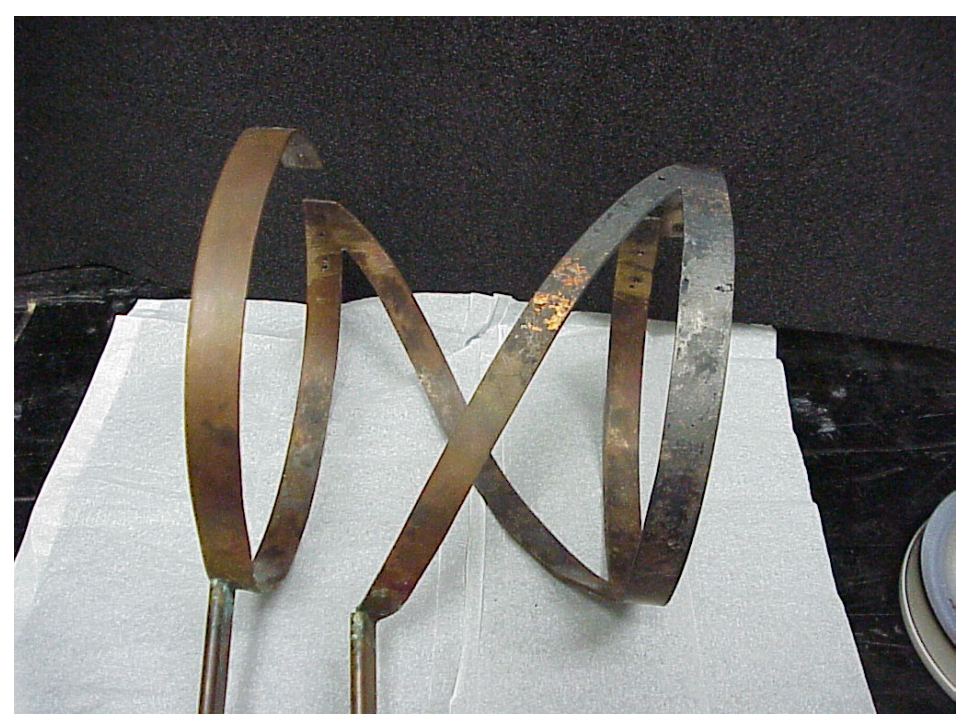

Figure 7: Evidence of asymmetric Ohmic heating of the $19 \mathrm{~cm}$ long $m=+1$ antenna used on HELIXd. The right side of the antenna is coated with a black film and no significant discoloration occurs on the left side.

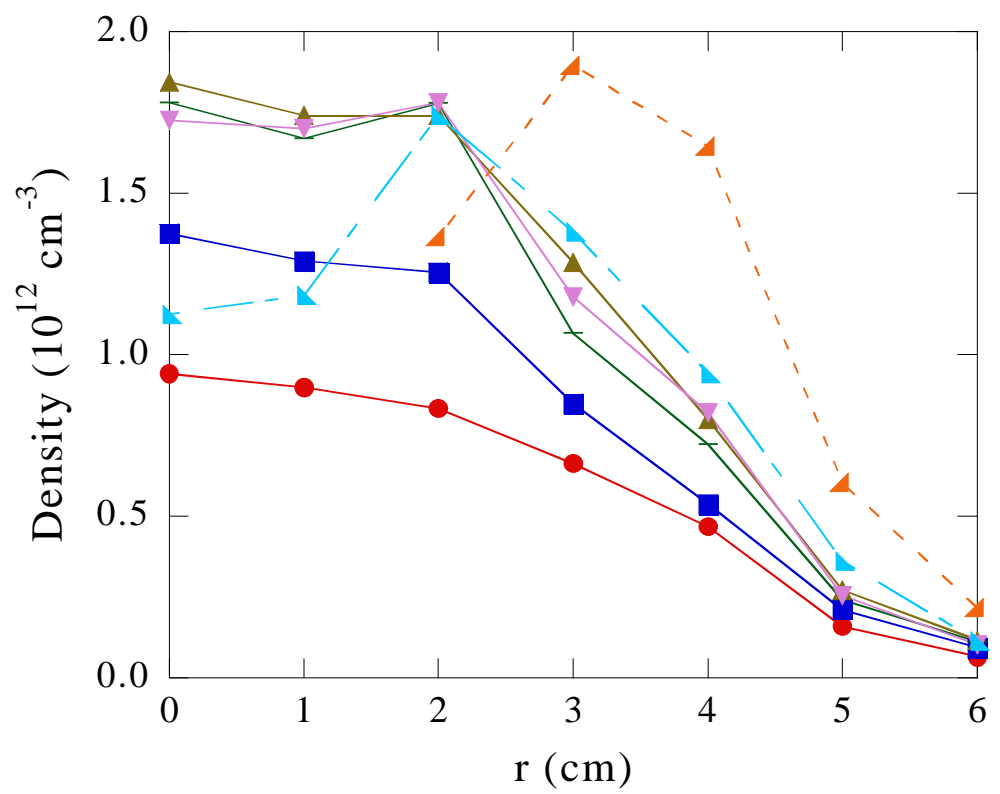

Figure 8: Density profiles for different magnetic field strength in HELIXc: $(\bullet) 462 \mathrm{G},(\boldsymbol{\bullet}) 540 \mathrm{G},(\diamond) 613$ $\mathrm{G},(\Delta) 687 \mathrm{G},(\boldsymbol{\nabla}) 761 \mathrm{G},(\Delta) 834 \mathrm{G}$, and $(\Delta) 915 \mathrm{G}$.

Experiments in HELIXc and HELIXd also yielded interesting measurements of the plasma density profile. In HELIXc, the plasma density profile measured with a Langmuir probe was often hollow when the RF driving frequency was below the lower hybrid frequency, $1 / \omega_{l h}^{2}=1 / \omega_{c e} \omega_{c i}+1 /\left(\omega_{p i}^{2}+\omega_{c i}^{2}\right)$, (Figure 8). Hollow density profiles 
have been observed in other experiments [Yun and Chang, 1998; Sakawa et al., 1999; Yun et al., 2001]. However, when the RF driving frequency was at 11 and $15 \mathrm{MHz}$, hollow profiles were also created even though the RF driving frequency was well above the lower hybrid frequency everywhere in the plasma. Figure 9 and Figure 10 show twodimensional, metastable ion density profiles measured with the two-dimensional LIF system along with a corresponding visible light image taken from the end of the source. Although the LIF signal intensity, i.e., the density of the metastable level ( $\left.\mathrm{n}_{(\text {ArII) }}\right)$ used for LIF, is not a direct measurement of the plasma density, it can be related to the plasma density (or electron density $n_{e}$ ) through

$$
\frac{d n_{(A r I I) m}}{d t}=n_{e}\left[\sum_{k} n_{(A r I) k}\langle\sigma v\rangle_{(i o n .+e x c .) k}+\sum_{l} n_{(A r I I) l}\langle\sigma v\rangle_{(e x c .) l}\right]+\sum_{j>m} A_{j m} n_{(A r I I) j},
$$

where $d n_{(A r I I) m} / d t$ is the LIF metastable creation rate, $n_{(A r I) k}$ are the populations of the different neutral argon levels, $n_{(A r I) l}$ are the populations of the different singly ionized argon levels, and $n_{(A r I) j}$ are the populations of excited argon ions with energy levels higher than the LIF metastable level. $\langle\sigma v\rangle_{(\text {ion.exc.)k }}$ are the production rate coefficients for LIF metastable ions associated with the different levels of neutral argon, and $\langle\sigma v\rangle_{(\text {exc.)l }}$ are the production rate coefficients for metastable ions associated with the different argon ion levels. The term $\sum_{j>m} A_{j m} n_{(A r I I) j}$ (or cascade term) represents the contribution to the LIF metastable ion population associated with de-excitation of highly excited ions. The energy states of these ion levels are higher than the LIF metastable energy state. These levels are also linked to the LIF metastable level by allowed transitions with tabulated transition probabilities $A_{j m}$ [Wiese et al., 1969]. For low electron temperatures, $T_{e}<8 \mathrm{eV}$, the populations of these states are negligible since the energies of the levels are greater than $20 \mathrm{eV}$ [Severn et al., 1998].

For specific plasma conditions (ionization fraction $\geq 25 \%, \mathrm{~T}_{\mathrm{e}}>5 \mathrm{eV}$ ), the contribution to the LIF metastable population from ion excitation $\left(2^{\text {nd }}\right.$ term) will be larger than the contribution from the combined excitation-ionization of neutral argon ( $1^{\text {st }}$ term) because some rate coefficients for ion excitation (especially excitation transfer) are much larger 
than the neutral excitation-ionization combined rates. Under these conditions, Equation (1.3) can be written as

$$
\frac{d n_{(A r I I) m}}{d t} \approx \sum_{l} n_{e} n_{(A r I I) l}\langle\sigma v\rangle_{(e x c .) l} .
$$

Thus, the LIF metastable density (or LIF intensity) is proportional to the square of the plasma density. Similarly, Blackwell and Chen [1997] have observed a correlation between $488 \mathrm{~nm}$ emission and plasma density in a helicon source.

The metastable ion density and visible light observations from HELIXc shown in Figure 9 and Figure 10 both clearly indicate hollow or "structured" density profiles. Since the hollow nature of the profile appears independent of the ratio of RF frequency to lower hybrid frequency, there results suggest that the hollow profiles are not strictly a result of the lower hybrid resonance. Since the lower hybrid resonance depends on the plasma density and the magnetic field strength (which is why it useful for plasma heating in Tokamaks [Stix, 1965; Chu et al., 1976]), the radial location of the peak should shift with changing magnetic field strength. Since this is not observed, these observations are in direct contradiction to assertions in recent publications [Yun et al., 2001]. Perhaps even more surprising is that all of the measured density profiles for RF frequencies above and below the lower hybrid frequency in HELIXd are peaked (Figure 11). Since the principal difference between HELIXc and HELIXd is the blocking of the plasma edge in HELIXc, the boundary conditions and not the lower hybrid resonance appear to play a key role in determining the density profile.

Besides the boundary conditions, HELIXc and HELIXd also have different tube diameters. For the same RF input power, the RF power density in HELIXd is $225 \%$ larger than in HELIXc. In other helicon experiments, the density profile has also been observed to change from hollow to peaked with increasing RF power [Yun et al., 2001]. Hollow profiles may simply be due to cylindrical symmetry and RF coupling that is more capacitive than "helicon." Since no two helicon sources are identical, it is difficult to identify what phenomena are common to helicon sources and what phenomena are due to source design and operational characteristics. For this reason, one of the objectives in 
this dissertation was to identify phenomena common to both HELIXd and HELIXb and interpret those common features in terms of a general model of helicon source physics.

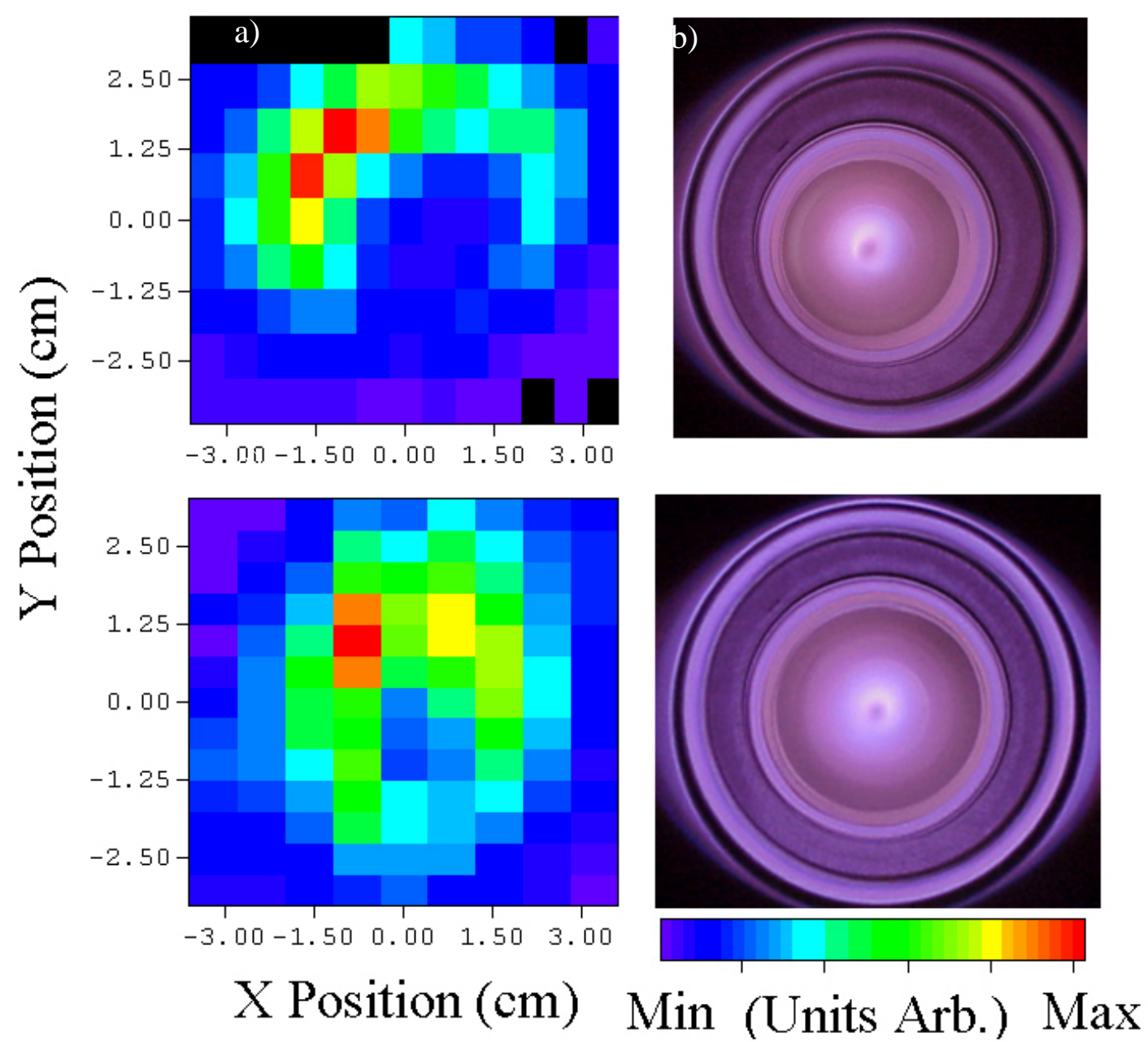

Figure 9: Measurements of the two-dimensional LIF signal intensity for an RF frequency of $15 \mathrm{MHz}$ and magnetic field strengths of a) $760 \mathrm{G}$ and c) $463 \mathrm{G}$. The corresponding visible light images looking along the axis of the source obtained with a CCD for b) $760 \mathrm{G}$ and d) $463 \mathrm{G}$. 

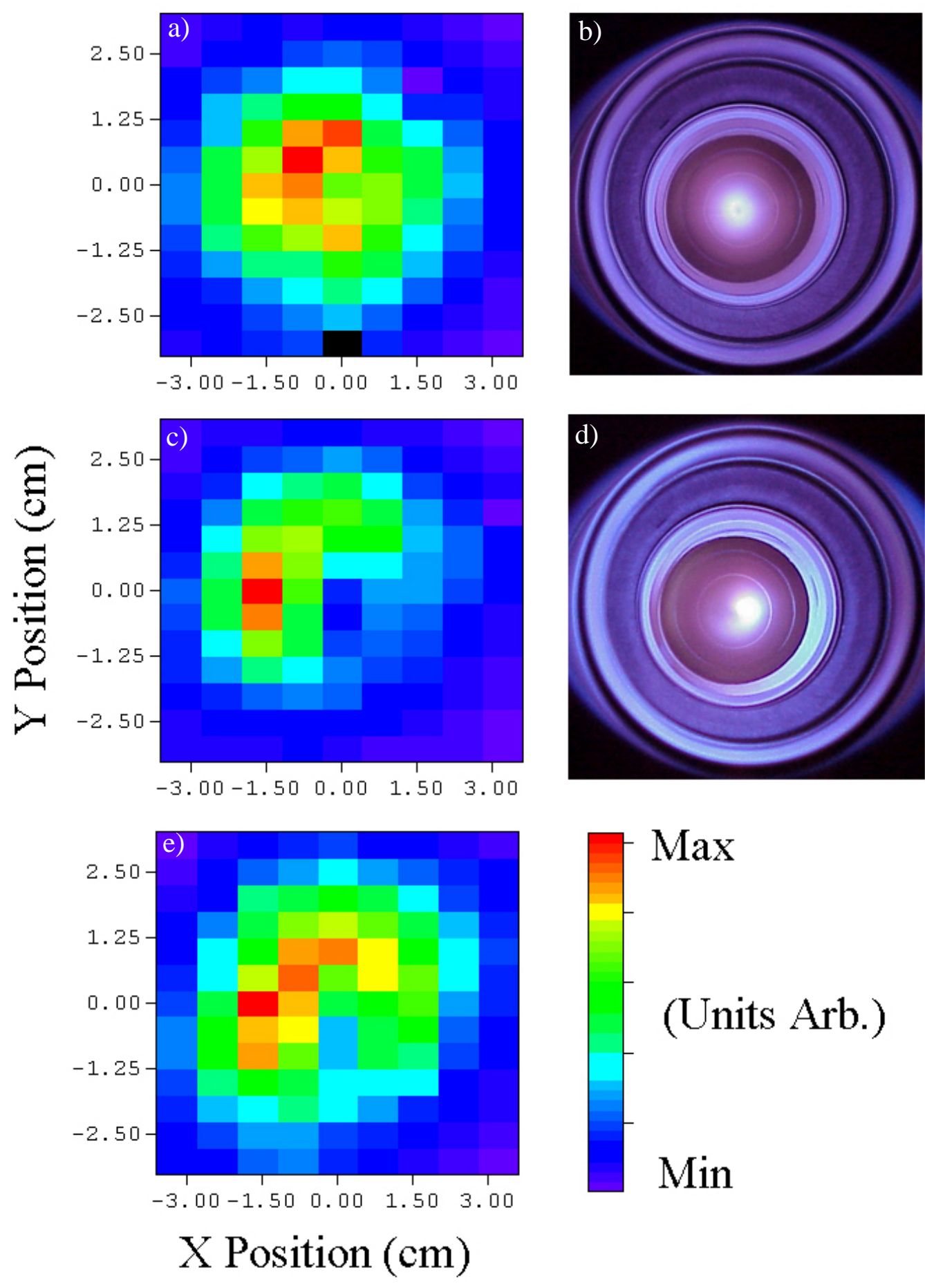

Figure 10: Measurements of the two-dimensional LIF signal intensity for an RF frequency of $11 \mathrm{MHz}$ and magnetic field strengths of a) $760 \mathrm{G}$, c) $615 \mathrm{G}$, and e) $463 \mathrm{G}$. The corresponding visible light images looking along the axis of the source obtained with a CCD for b) $760 \mathrm{G}$ and d) $615 \mathrm{G}$. 


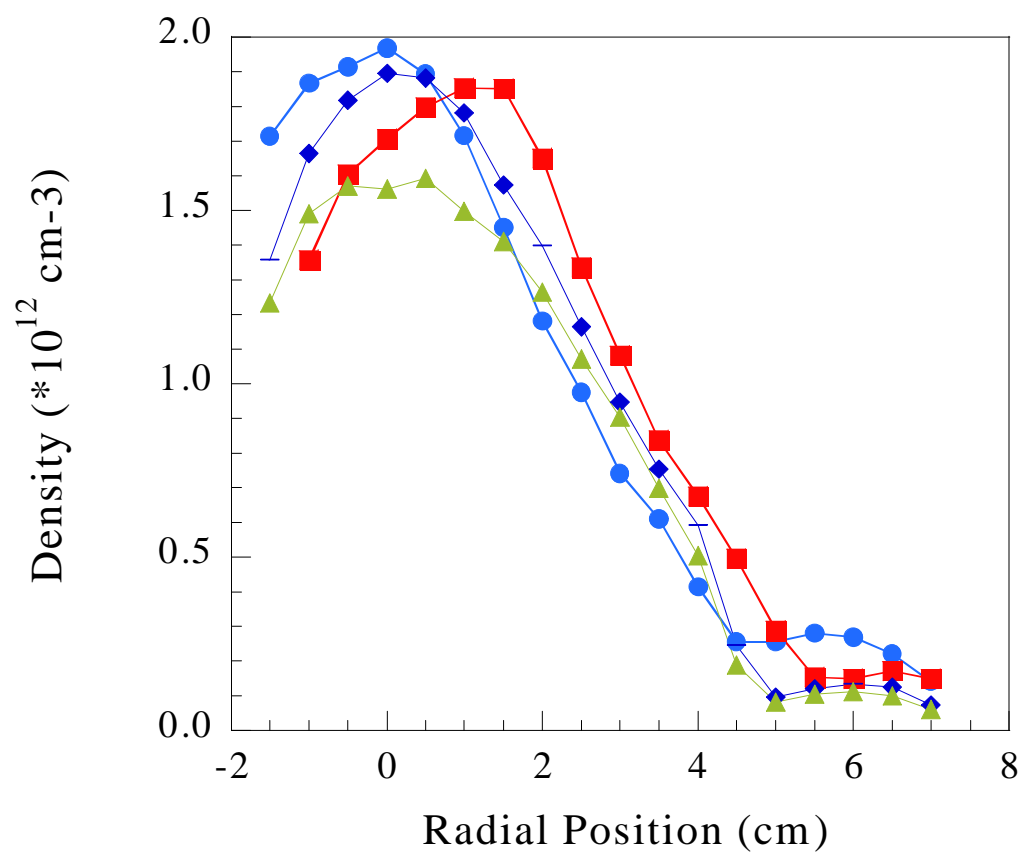

Figure 11: Radial density profiles in HELIXd for magnetic field strengths of ( $\bullet$ ) $663 \mathrm{G},(\bullet) 807 \mathrm{G},(\bullet)$ $948 \mathrm{G}$, and $(\triangle) 1089 \mathrm{G}$. The operating parameters were a RF frequency of $9 \mathrm{MHz}$, a neutral pressure of 6.7 mTorr, and a RF Power of 750 Watts.

\subsection{Active Areas of Helicon Source Research.}

At a recent meeting of the American Physical Society's Division of Plasma Physics, Boswell identified several research areas needing exploration before a complete theoretical model of the helicon source could be constructed [Boswell, 2001]:

- Power balance and efficiency

- Electron inertia, resonance cones, and Trivelpiece-Gould modes

- Lower hybrid resonance effects

- Diffusion across the magnetic field

- Ion heating

- Neutral pumping

- Electron-wave interactions

- Wave-wave (parametric) interactions 
As mentioned previously, ion heating in helicon sources has been a research topic at WVU for a number of years. In this work, it will be shown that slow waves, the so-called Trivelpiece-Gould modes, the lower hybrid resonance, and wave-wave interactions all play important roles in the ion heating process. As shown by Balkey [2000], the ion heating rate is too large to be due to electron-ion collisions, i.e., the time needed for the electrons to heat the ions to the measured ion temperatures is larger than the ion confinement time. The observed ion temperature anisotropies are also inconsistent with electron-ion thermal equilibration through collisions. Such collisional heating would yield isotropic ions. Thus, the only possible ion heating mechanisms are direct particlewave interactions, i.e., Landau damping, or non-linear processes such as stochastic ion heating. Balkey also showed a clear correlation between ion heating, density production, and the lower hybrid frequency [2000]. However, the actual mechanism responsible for the ion heating and enhanced density production was not identified. The primary focus of the research described in this dissertation is to identify the mechanism responsible for ion heating in helicon sources. Three possible heating mechanisms that could yield large perpendicular ion temperatures at RF frequencies near the lower hybrid frequency are: ion Landau damping of slow waves which have a resonance at the lower hybrid frequency, $k_{\perp} \rightarrow \infty$ [Cho, 2000]; preferential scattering of ions by short wavelength ion acoustic turbulence nonlinearly driven in the near field of the RF antenna (the predicted maximum ion heating rate occurs for RF frequencies just above the lower hybrid frequency) [Akhiezer et al., 1998]; and ion heating by parametrically driven low frequency waves, e.g., ion acoustic, ion cyclotron, and ion Bernstein waves. Since damped parametrically driven waves and electron scattering by ion acoustic turbulence are also mechanisms whereby RF energy can be coupled into electrons, such mechanisms could also explain the correlation of plasma density with the lower hybrid frequency. Given that there have been many experimental observations of parametrically driven instabilities with large growth rates for pump frequencies near the lower hybrid frequency [Porkolab et al., 1972; Brusati et al., 1974; Wong and Ono, 1981], it is reasonable to assume that similar processes could occur in helicon sources operating at RF frequencies near the lower hybrid frequency. 


\section{Chapter 1 References}

Aigrain, P., Les 'helicons' dans les semiconducteurs, Procedures of the International Conference on Semiconductors Physics, 224 (1960).

Akhiezer, A.I., Mikhailenko, V.S., and Stepanov, K.N., Ion-sound parametric turbulence and anomalous electron heating with application to helicon plasma sources, Phys. Lett. A, 245, 117 (1998).

Balkey, M.M., Optimization of a Helicon Plasma Source for Maximum Density with Minimal Ion Heating, Ph. D. thesis, West Virginia University, Morgantown (2000).

Balkey, M.M., Boivin, R.F., Kline, J.L., and Scime, E.E., Ion heating and density production in helicon sources near the lower hybrid frequency, Plasma Sources Sci. Technol., 10, 284 (2001).

Blackwell, D.D., and Chen, F.F., Two-Dimensional Imaging of a Helicon Discharge, Plasma Sources Sci. Technol., 6, 569 (1997).

Boivin, R.F., Kline, J.L., Scime, E.E., and Cohen, S.A., Simple and Inexpensive Diode Base LIF diagnostic for Ar Plasmas, Rev. Sci. Instrum., to be submitted (2002).

Boswell, R., Perspective on Current Helicon Source Science Issues, Bulletin of the American Physical Society, 46, 30 (2001).

Boswell, R.W., Plasma production using a standing helicon wave, Phys. Lett. A, 33, 457 (1970).

Boswell, R.W., Measurements of the far-field resonance cone for whistler mode waves in a magnetoplasma, Nature, 258, 58 (1975a).

Boswell, R.W., Non-convective parametric instability associated with whistler wave resonance cone, Phys. Lett. A, 55A, 93 (1975b).

Boswell, R.W., and Chen, F.F., Helicons-the early years, IEEE Trans. Plasma Sci., 25, 1229 (1997).

Brusati, M., Cima, G., Fontanesi, M., and Sindoni, E., Lower hybrid resonance and parametric decay excited by a slow-wave device, Lettere al Nuovo Cimento, 10, ser.2, 67 (1974).

Chang Diaz, F.R., Goulding, R.H., Bengtson, R.D., Wally Baity, F., Sparks, D., Bussell, R.G., Jr., Barber, G.C., McCaskill, G., Jacobson, V.T., Carter, M.D., Ilin, A.V., and Glover, T.W., Experimental status of the development of a variable specific impulse magnetoplasma rocket, Fusion Technology, 35, 243 (1999).

Chen, F.F., Plasma ionization by helicon waves, Plasma Phys. Controlled Fusion, 33, 339 (1991).

Chen, F.F., Experiments on helicon plasma sources, J. Vac. Sci. Technol. A, 10, 1389 (1992).

Chen, F.F., and Arnush, D., Generalized theory of helicon waves. I. Normal modes, Phys. Plasmas, 4, 3411 (1997).

Chen, F.F., and Boswell, R.W., Helicons-the past decade, IEEE Trans. Plasma Sci., 25, 1245 (1997).

Chen, F.F., and Decker, C.D., Electron acceleration in helicon sources, Plasma Phys. Controlled Fusion, 34, 635 (1992).

Chen, F.F., Jiang, X., Evans, J.D., Tynan, G., and Arnush, D., Low-field helicon discharges, Plasma Phys. Controlled Fusion, 39, 411 (1997). 
Chevalier, G., and Chen, F.F., Experimental modeling of inductive discharges, Journal of Vacuum Science and Technology A, 11, 1165 (1993).

Cho, S., The role of the lower hybrid resonance in helicon plasmas, Phys. Plasmas, $\mathbf{7}$, 417 (2000).

Chu, C., Dawson, J.M., and Okuda, H., Plasma heating at frequencies near the lower hybrid, Phys. Fluids, 19, 981 (1976).

Cohen, S.A., Berlinger, B., Corso, V., Fahmy, F., Gorman, J., Levinton, F., Lemunyan, G., and Fredriksen, A., The magnetic nozzle experiment, Bulletin of the American Physical Society, 44 (1999).

Franck, C., Private Communication, 2001.

Franck, C., and Klinger, T., Helicons - Our Last Year, Bulletin of the American Physical Society, 46 (2000).

Gary, S.P., Anderson, B.J., Denton, R.E., Fusilier, S.A., and McKean, M.E., A limited closer relation for anisotropic plasmas from the Earth's magnetosheath, Phys. Plasmas, 1, 1676 (1994a).

Gary, S.P., McKean, M.E., Winske, D., Anderson, B.J., Denton, R.E., and Fusilier, S.A., The proton cyclotron instability and the anisotropy/beta inverse correlation, $J$. Geophys. Res., 99, 5903 (1994b).

Grulke, O., Greiner, F., Klinger, T., and Piel, A., Comparative experimental study of coherent structures in a simple magnetized torus, Plasma Phys. Controlled Fusion, 43, 525 (2001).

Hanna, J., and Watts, C., Alfvén wave propagation in a helicon plasma, Phys. Plasmas, 8, 4251 (2001).

Hill, D.H., Fornaca, S., and Wickham, M.G., Single frequency scanning laser as a plasma diagnostic, Rev. Sci. Instrum., 54, 309 (1983).

Keiter, P.A., Experimental Investigation of Ion Temperature Anisotropy Driven Instabilities in a High Beta Plasma, Ph. D. thesis, West Virginia University, Morgantown (1999).

Keiter, P.A., Scime, E.E., and Balkey, M.M., Frequency dependent effects in helicon plasmas, Phys. Plasmas, 4, 2741 (1997).

Kline, J.L., Scime, E.E., Keiter, P.A., Balkey, M.M., and Boivin, R.F., Ion heating in the HELIX helicon plasma source, Phys. Plasmas, 6, 4767 (1999).

Kwak, J.G., Choi, H.D., Bak, H.I., Suwon, C., Bak, J.G., and Kim, S.K., Frequency dependence of the plasma density for helicon plasmas, Phys. Plasmas, 4, 1463 (1997).

Lehane, J.A., and Thonemann, P.C., An Experimental Study of Helicon Wave Propagation in Gaseous Plasma, Procedures of the Physics Society, 85, 103 (1965).

Light, M., Chen, F.F., and Colestock, P.L., Low frequency electrostatic instability in a helicon plasma, Phys. Plasmas, 8, 4675 (2001).

Loewenhardt, P.K., Blackwell, B.D., Boswell, R.W., Conway, G.D., and Hamberger, S.M., Plasma production in a toroidal heliac by helicon waves, Phys. Rev. Lett., 67, 2792 (1991).

McKean, M.E., Winske, D., and Gary, S.P., Two-dimensional simulations of ion anistoropy instabilities in the magnetosheath, J. Geophys. Res., 99, 11 (1994). 
Nakano, T., Gottscho, R.A., Sadeghi, N., Trevor, D.J., Boswell, R.W., Perry, A.J., Lee, T.C., Giapis, K.P., and Margot, J., Helicon wave excited plasmas, Oyo Buturi, 61, 711 (1992).

Perry, A.J., Vender, D., and Boswell, R.W., The application of the helicon source to plasma processing, Journal of Vacuum Science \& Technology B (Microelectronics Processing and Phenomena), 9, 310 (1991).

Porkolab, M., Arunasalam, V., and Ellis, R.A., Jr., Parametric instability and anomalous heating due to electromagnetic waves in plasma, Phys. Rev. Lett., 29, 1438 (1972).

Sakawa, Y., Takino, T., and Shoji, T., Contribution of slow waves on production of highdensity plasmas by $m=0$ helicon waves, Phys. Plasmas, 6, 4759 (1999).

Scime, E.E., Keiter, P.A., Balkey, M.M., Bolvin, R.F., Kline, J.L., Blackburn, M., and Gary, S.P., Ion temperature anisotropy limitation in high beta plasmas, Phys. Plasmas, 7, 2157 (2000).

Scime, E.E., Keiter, P.A., Zintl, M.W., Balkey, M.M., Kline, J.L., and Koepke, M.E., Control of ion temperature anisotropy in a helicon plasma, Plasma Sources Sci. Technol., 7, 186 (1998).

Severn, G.D., Edrich, D.A., and McWilliams, R., Argon ion laser-induced fluorescence with diode lasers, Rev. Sci. Instrum., 69, 10 (1998).

Shoji, T., Sakawa, Y., Nakazawa, S., Kadota, K., and Sato, T., Plasma production by helicon waves, Plasma Sources Sci. Technol., 2, 5 (1993).

Stern, R.A., and Johnson, J.A., III, Plasma ion diagnostics using resonant fluorescence, Phys. Rev. Lett., 34, 1548 (1975).

Stix, T.H., Radiation and Absorption Via Mode Conversion in an Inhomogeneous Collision Free Plasma, Phys. Rev. Lett., 15, 878 (1965).

Sudit, I.D., and Chen, F.F., RF compensated probes for high-density discharges, Plasma Sources Sci. Techol., 3, 162 (1994).

Sun, X., Boivin, R., Keesse, A., Kline, J., Scime, E., Spangler, R., and Woehrman, M., Flow Measurments in a Helicon Plasma Source, Bulletin of the American Physical Society, 46, 59 (2001).

Wiese, W.L., Bridges, J.M., Kornblith, R.L., and Kelleher, D.E., Transition probabilities for prominent Ar I lines in the near infrared, Journal of the Optical Society of America, 59, 1206 (1969).

Wong, K.L., and Ono, M., Parametric decay of lower-hybrid waves in the ACT-1 toroidal device, Phys. Rev. Lett., 47, 842 (1981).

Yun, S., Suwon, C., Tynan, G., and Hongyoung, C., Density enhancement near lower hybrid resonance layer in $m=0$ helicon wave plasmas, Phys. Plasmas, 8, 358 (2001).

Yun, S.M., and Chang, H.Y., Radial density profile change near lower hybrid frequency in $m=0$ helicon wave plasmas, Phys. Lett. A, 248, 400 (1998). 


\section{Chapter 2: Experimental Setup}

The combined HELIX (Hot hELIcon eXperiment) and LEIA (Large Experiment on Instabilities and Anisotropies) system was designed to study high beta $\left(\beta=n k_{b} T \mu_{o} / B^{2}\right)$ plasmas as are found in space. The combination, seen in Figure 12, takes advantage of the high-density plasmas generated by HELIX and the small magnetic fields in LEIA to create a high beta plasma. The large diameter of the LEIA chamber enables the plasmas to be fully magnetized. The high beta plasmas in LEIA can then be used to study magnetospherically relevant plasma physics in the laboratory.

In this Chapter, the details of the experimental apparatus are described. For these experiments, the primary focus is on the physics in HELIX. Since HELIX and LEIA are connected, the experiment is described as a whole although the LEIA parameters were held fixed at all times. General descriptions of HELIX and LEIA can also be found in Keiter [1999], Balkey [2000], Kline et al. [1999], and Scime et al. [2000].

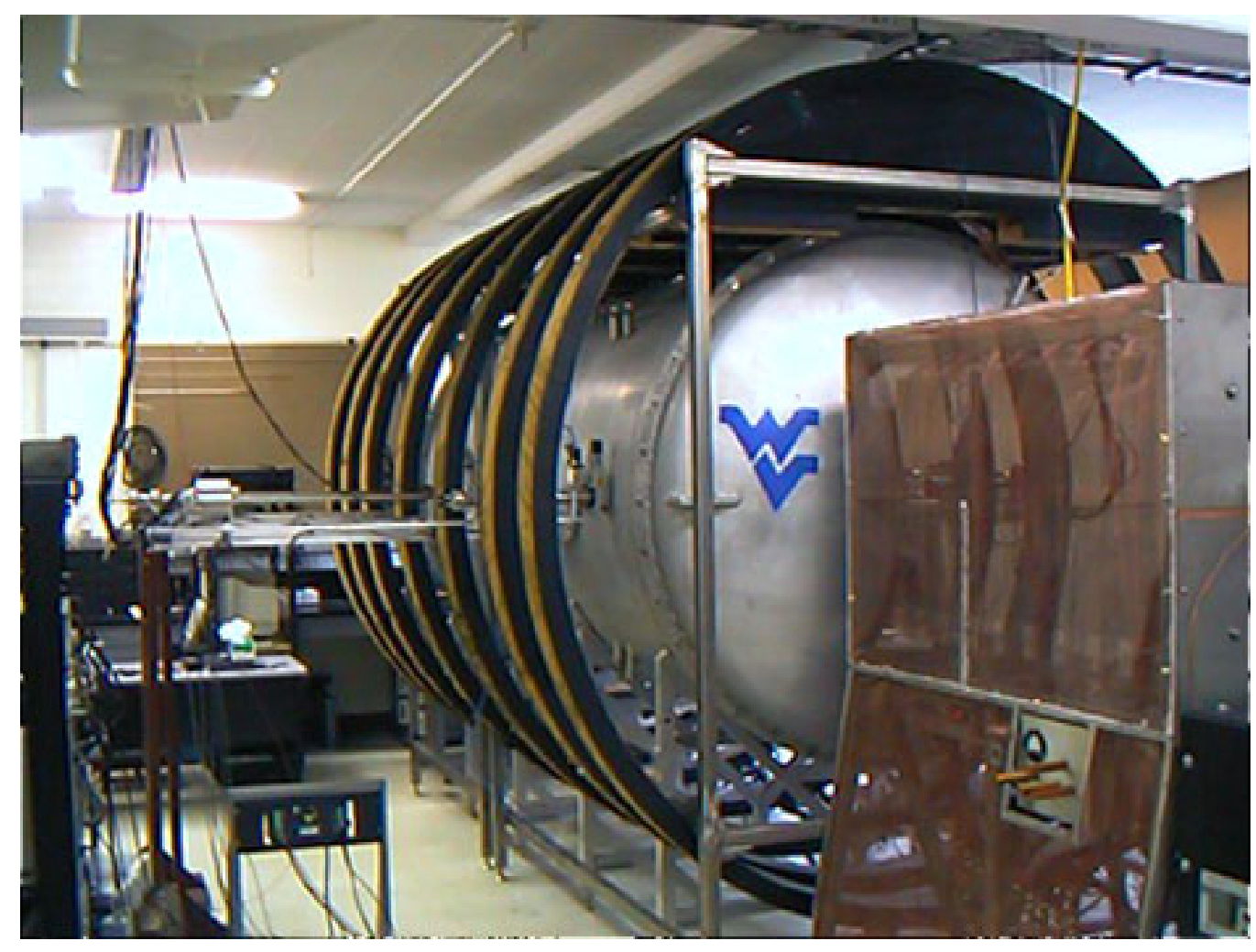

Figure 12: HELIX (foreground) and LEIA (large aluminum chamber). HELIX resides inside a Faraday cage (copper screening). The large electromagnets surrounding LEIA are approximately $3 \mathrm{~m}$ in diameter. 


\subsection{The Plasma Chamber}

The experiment consists of several sections. Starting from the HELIX end, there is a glass tee connected to the pumping station and to a 12 " diameter stainless steel flange. The stainless steel flange, which includes the gas inlet for the source, connects to a 4" OD 24" long Pyrex tube. The Pyrex section of the chamber is for coupling power from the RF antenna into the plasma. The Pyrex tube is connected to a 6" ID, 36" long stainless steel chamber with one set of four 6" Conflat ${ }^{\mathrm{TM}}$ crossing ports and four sets of four $23 / 4$ Conflat $^{\mathrm{TM}}$ crossing ports. The $23 / 4$ Conflat $^{\mathrm{TM}}$ crossing ports are spaced evenly on either side of the four 6" crossing ports. A schematic view of the plasma chamber is shown again in Figure 13. Unlike previous experiments [Keiter, 1999; Balkey, 2000; Scime et al., 2000], the stainless steel chamber is directly connected to the space simulation plasma chamber. The space simulation chamber, LEIA is large aluminum cylindrical chamber on loan from Massachusetts Institute of Technology (MIT). LEIA has an inner diameter of $1.8 \mathrm{~m}$ and a length of $4.4 \mathrm{~m}$. The LEIA pumping station is connected to the far end of LEIA.

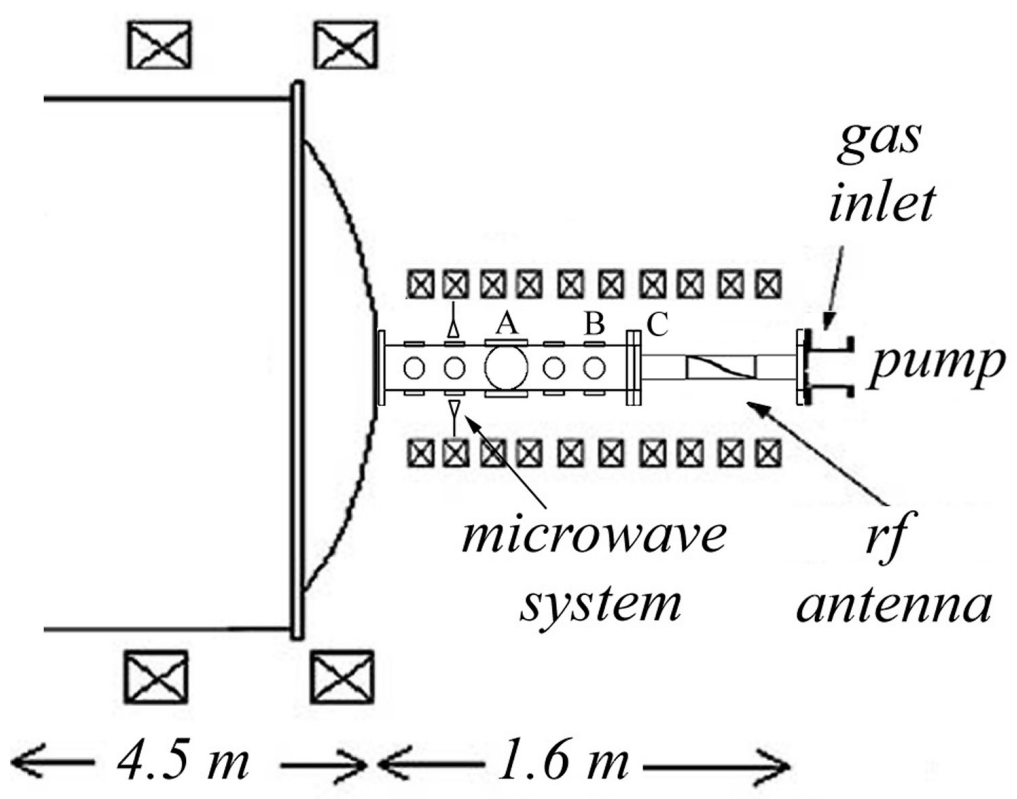

Figure 13: Schematic diagram of the HELIX plasma chamber. 


\subsection{Vacuum System}

The vacuum for the experiments is maintained by three turbomolecular drag pumps. The HELIX pumping station consists of a Balzer's TMU 520 turbomolecular drag pump connected to a Balzer's MD 4T diaphragm roughing pump. The pumps were separated from the glass cross by a MDC GV-4000M-P 6 inch ID (inner diameter) gate valve. The pumping station at the far end of LEIA has two Pfeiffer TMU 1600 turbomolecular pumps with Pfeiffer MD-8 backing pumps. Each set of pumps is separated from LEIA by a MDC GV-8000V-P 10" gate valve. All three gate valves are connected to a failsafe interlock circuit that automatically shuts the gate valves upon power failure or high pressure indication. A base pressure of $3.0 \times 10^{-7}$ Torr is maintained with all three pumps running in low speed. The pressure is measured by two Balzers PKR250 full range pressure gauges with Balzers TPG252 dual gauge controllers. The full range gauges consist of a Pirani gauge for high pressures ( $>10^{-2}$ Torr) and a cold cathode gauge for low pressures $\left(<10^{-2}\right.$ Torr). One is located on the glass tee of the HELIX pumping station and the other is located on LEIA near the LEIA pumping station. For gas flow control, a MKS1179 mass flow valve with a PR-4000 flow controller is used to maintain the neutral pressure at the desired value.

\subsection{The Magnetic field}

The magnetic field for HELIX is generated by ten electromagnets donated by the Max Planck Institüt in Garching, Germany. The magnets have 46 internal copper windings with a resistance of $17 \mathrm{~m} \Omega$ and an inductance of $1.2 \mathrm{mH}$. The magnets are water-cooled and roll on a pair of rails that allow their axial positions to be adjusted. A Macroamp 400 Amp power supply provides the current for the electromagnets. The magnetic field for LEIA is generated by seven, custom built 9' diameter electromagnets. Each water-cooled magnet contains 20 turns (2 layers of 10 turns) of $0.36 "$ x 0.41 " hollow rectangular aluminum tubing. With a 200 Amp DC EMHP power supply, the LEIA magnetic field ranges from 0 to 70 Gauss. Unless otherwise stated, for all of the experiments reported in this work, the current was fixed at 100 Amps, i.e. 35 Gauss.

The HELIX electromagnets were used in two different magnetic field configurations. One was a uniform field using all ten magnets (ten-coil configuration). The other 
configuration used only the first eight magnets (eight-coil configuration) and the last two magnets between HELIX and LEIA were disconnected. The eight-coil configuration was used to obtain higher magnetic field strengths by reducing the resistance of the magnet set (the available power supply was voltage limited). Using only eight coils allowed more than a 60 Amp increase in current or 200 Gauss increase in magnetic field strength. Figure 14 shows the axial magnetic strength profile for both configurations with the current in the HELIX magnets at 160 Amps. The downstream magnetic field strength drops faster with eight HELIX coils than with ten HELIX coils. Figure 15 shows a more detailed view of the difference in the two configurations by expanding the HELIX portion of the plot.

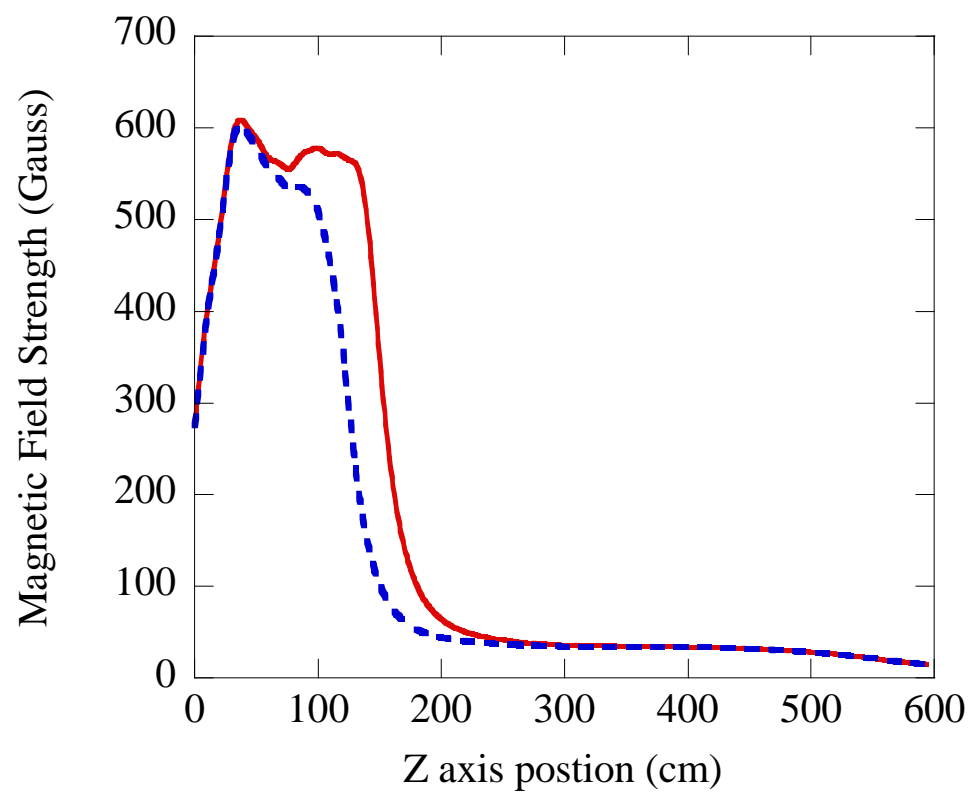

Figure 14: Axial magnetic field for HELIX and LEIA as a function of $z$ with a current of 160 Amps in the HELIX magnets and 100 Amps in the LEIA magnets for $(-)$ the ten-coil configuration and (...) the eightcoil configuration. 


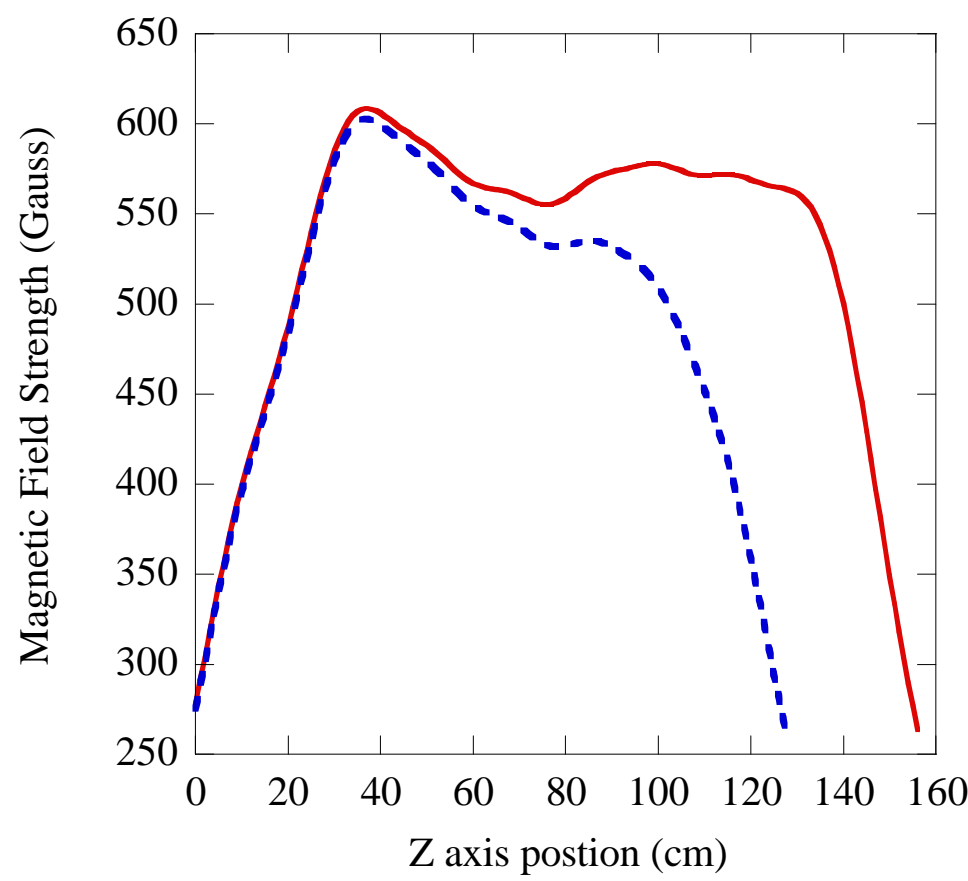

Figure 15: Axial magnetic field strength profile for ( - ) the ten-coil configuration and $(\cdots \cdots)$ the eight-coil configuration in HELIX as a function of $\mathrm{z}$ with a current of 160 Amps in the HELIX magnets and 100 Amps in the LEIA magnets.

The axial magnetic field strength profiles in HELIX for the maximum amount of current in each of the two configurations is shown in Figure 16.

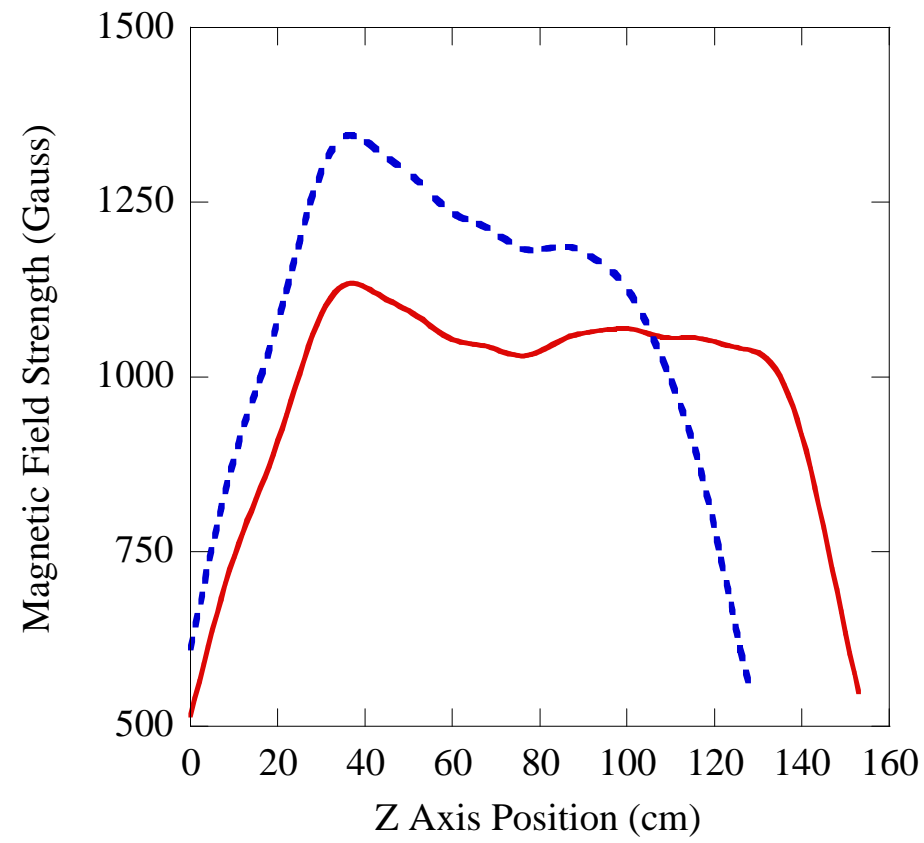

Figure 16: Maximum magnetic field strength profiles in HELIX for $(-) 300$ amps in ten coils and for (...) 360 amps in eight coils. 


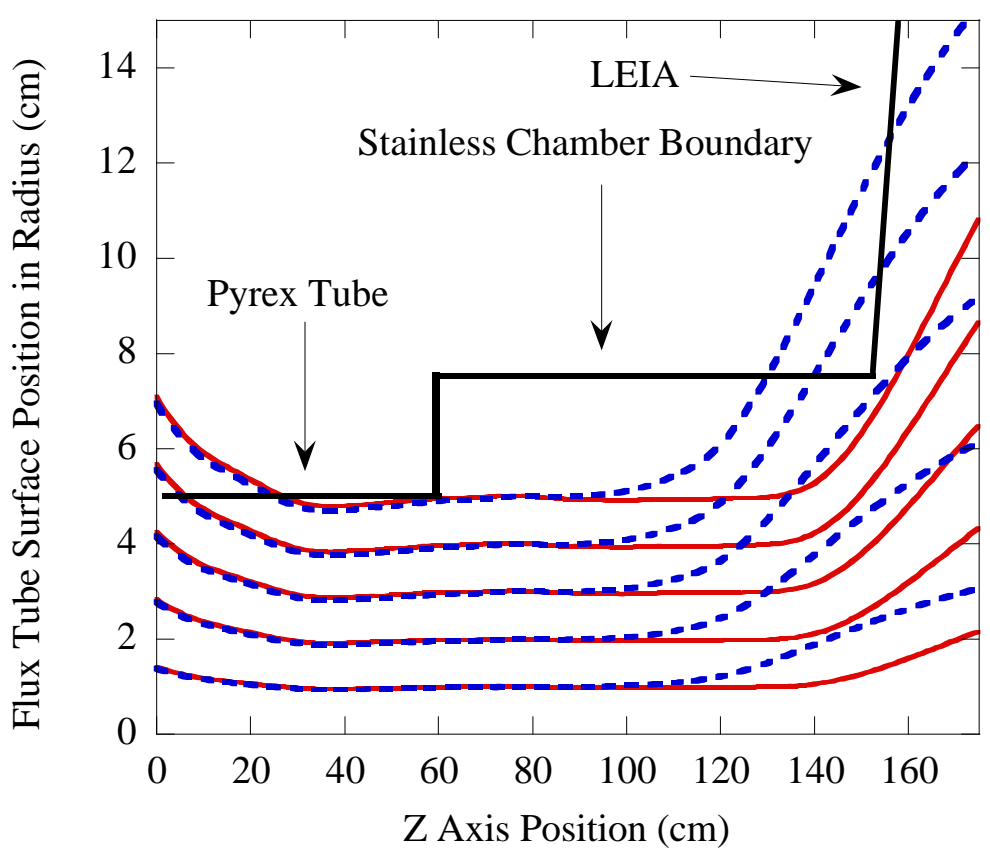

Figure 17: Flux tubes at radii of $1-5 \mathrm{~cm}$ with a $1 \mathrm{~cm}$ separation for the (-) ten-coil and ( $\cdots . \cdot)$ eight-coil configurations for a current of $160 \mathrm{amps}$ in the HELIX magnets.

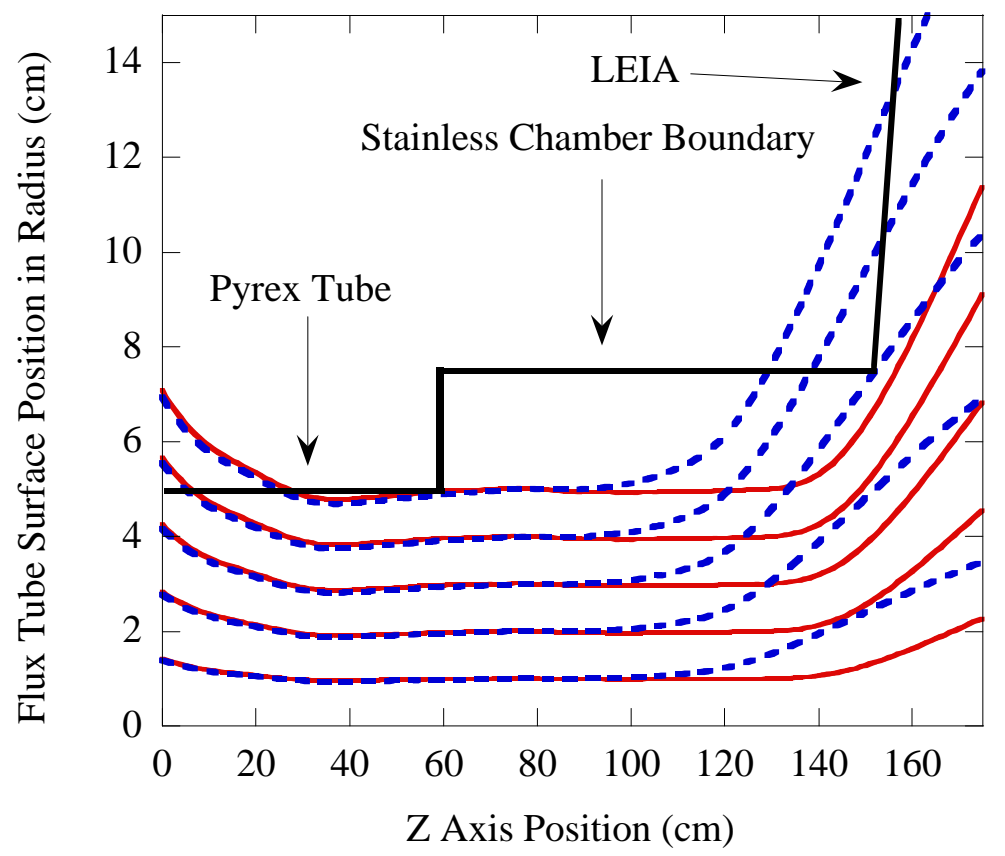

Figure 18: Flux tubes at radii of $1-5 \mathrm{~cm}$ with a $1 \mathrm{~cm}$ separation for the (-) ten-coil and $(\cdots .$.$) eight-coil$ configurations for a current of 300 amps in the HELIX magnets. 
One important issue in helicon experiments is the axial boundary condition. Figure 17 and Figure 18 show flux tubes (curves of constant magnetic flux) in HELIX for currents of 160 and 300 Amps respectively. The flux tubes are based on the magnetic field strength at an axial position of $z=80 \mathrm{~cm}$ and radial positions of 1 to $5 \mathrm{~cm}$ in $1 \mathrm{~cm}$ increments. The plasma radius is $5 \mathrm{~cm}$. In the ten-coil configuration, the outermost flux tubes do not intersect the HELIX stainless steel chamber wall near the LEIA boundary and continue into LEIA. In the eight-coil configuration, the outer flux tubes at radii between 3 and $5 \mathrm{~cm}$ do intersect the HELIX stainless steel chamber wall. In both the tencoil and eight-coil configurations, the flux tubes intersect the Pyrex tube on the backside of the antenna near $z=30 \mathrm{~cm}$. Intersection of the field lines with the Pyrex tube should not create a significant effect on the plasma since the tube is a nonconductor. The Pyrex surface will charge to the floating potential and no current will flow to the wall along the field. However, the intersection of the field lines with the HELIX stainless steel chamber wall can have a much more significant effect. The potential along the field lines that intersect the wall is set to ground at that end of the field lines. If the plasma potential is not equal to the chamber potential, a field-aligned current will flow to the wall in the eight-coil configuration. This boundary condition could also affect any waves propagating along the edge magnetic field lines. Due to the conducting boundary conditions, the electric fields parallel to the chamber wall must be zero at the chamber wall. Thus, the intersection of the outer field lines with the conducting stainless steel chamber wall in the eight-coil configuration may create finite length cavity modes in the helicon source.

\subsection{RF Generation and matching}

The plasma in a helicon source is created by a strong RF field coupled to the plasma through a dielectric glass wall. For HELIX, an ENI $200030 \mathrm{~dB}$ amplifier delivers up to $2 \mathrm{~kW}$ of RF power at a frequency of $0.3-35 \mathrm{MHz}$ to create the RF fields for the plasma source. The input signal for the amplifier comes from a $50 \mathrm{MHz}$ Wavetek function generator. The amplifier output is coupled to the antenna through a $\pi$-matching network to match the inductive load of the antenna to the amplifier's real output impedance of 50 Ohms. The matching network consists of one load and three tuning capacitors. The load 
capacitor is a Jennings high voltage tunable (20-2000 pF) capacitor. The three tuning capacitors are all Jennings high voltage tunable capacitors, two of which have a range of 4-250 $\mathrm{pF}$ and the third has a range of 5-500 pF. The matching capacitors are connected as shown in Figure 19. The capacitors are connected by sheets of copper inside the matching box that are internally connected to the antenna by silver plated copper rods. These connections are chosen to minimize the contact resistance and power losses to the antenna. Notice that the circuit is designed so that the antenna has no ground, i.e. a balanced antenna. The result is an average potential on the antenna larger than ground by the amount of potential across the three tuning capacitors. Without a ground reference, the potential on the antenna oscillates about the plasma potential [Balkey, 2000].

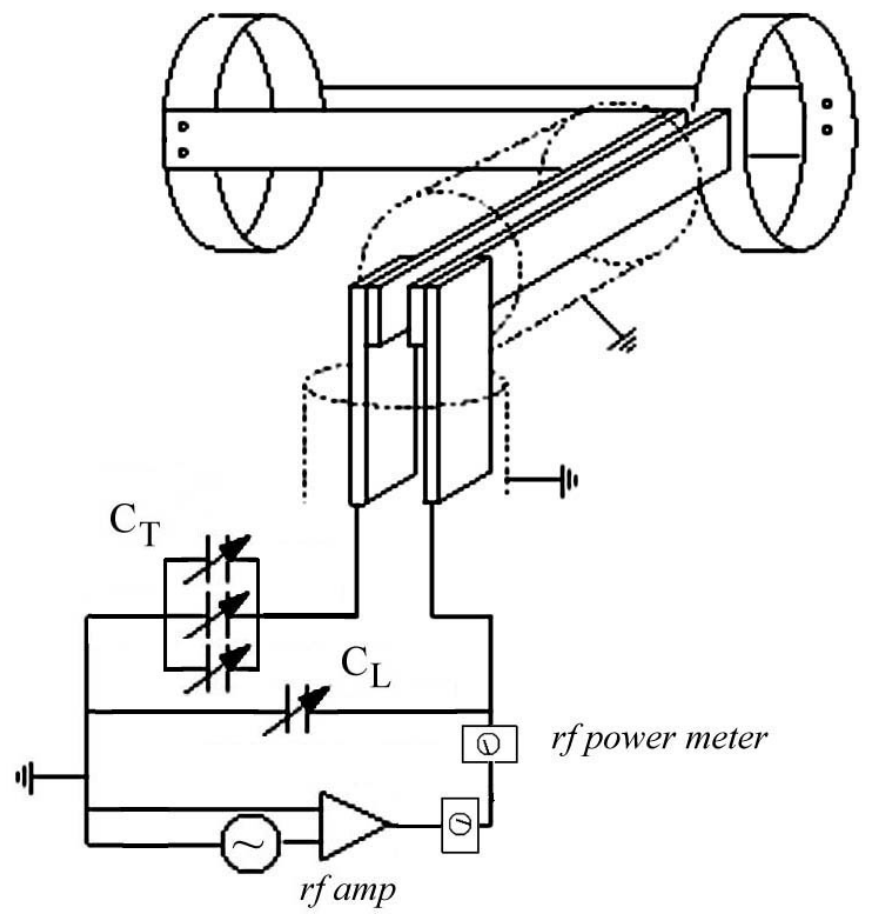

Figure 19: Antenna matching circuit for HELIX [Balkey, 2000]. $C_{T}$ is the tuning capacitor and $C_{L}$ is the load capacitor.

\subsection{Operational parameters}

HELIX can be operated over a wide range of magnetic field strengths, neutral fill pressures, RF powers, and RF frequencies. The wide range of parameters makes comparison with other helicon sources difficult. For comparison with previous WVU 
experiments, a narrow parameter range was selected for these experiments: the RF Power was fixed at 750 Watts, the neutral pressure was fixed at 6.7 mTorr, and the LEIA magnetic field strength was fixed at 35 Gauss. These experiments focused on varying the magnetic field strength and the RF driving frequency to investigate lower hybrid effects. Other typical operating parameters for these experiments are listed in Table 1.

Table 1: Standard operating parameters for HELIX.

\begin{tabular}{|c|c|}
\hline Parameter & Typical HELIX Values \\
\hline Gas Species & Argon \\
\hline Base Pressure & $<2 \times 10^{-7}$ Torr \\
\hline Operating Pressure & $6.7 \mathrm{mTorr}$ \\
\hline Magnetic Field & $<1300 \mathrm{G}$ \\
\hline RF Power & $750 \mathrm{~W}$ \\
\hline Operating Frequency & $7.5-16 \mathrm{MHz}$ \\
\hline Density & $\leq 2 \times 10^{13} \mathrm{~cm}^{-3}$ \\
\hline Electron Temperature & $\sim 5 \mathrm{eV}$ \\
\hline Ion Temperature & $<1 \mathrm{eV}$ \\
\hline Electron Gyroradius & $\sim .04 \mathrm{~mm}$ \\
\hline Ion Gyroradius & $\sim 2.7 \mathrm{~mm}$ \\
\hline
\end{tabular}




\section{Chapter 2 References}

Balkey, M.M., Optimization of a Helicon Plasma Source for Maximum Density with Minimal Ion Heating, Ph. D. thesis, West Virginia University, Morgantown (2000).

Keiter, P.A., Experimental Investigation of Ion Temperature Anisotropy Driven Instabilities in a High Beta Plasma, Ph. D. thesis, West Virginia University, Morgantown (1999).

Kline, J.L., Scime, E.E., Keiter, P.A., Balkey, M.M., and Boivin, R.F., Ion heating in the HELIX helicon plasma source, Phys. Plasmas, 6, 4767 (1999).

Scime, E.E., Keiter, P.A., Balkey, M.M., Bolvin, R.F., Kline, J.L., Blackburn, M., and Gary, S.P., Ion temperature anisotropy limitation in high beta plasmas, Phys. Plasmas, 7, 2157 (2000). 


\section{Chapter 3: Diagnostics}

The discussion of each of the diagnostics used for the experiments described in this dissertation is divided into three sections: theory, design, and hardware. Although none of the diagnostic techniques used are completely original in concept, many features of the experimental hardware are unique to HELIX.

\subsection{Laser Induced Fluorescence}

After the development of lasers in the 1960s, many new spectroscopic measurement techniques were developed. One such technique, laser induced fluorescence (LIF), uses a laser tuned to a natural absorption line of an atom or ion to induce emission from the upper pumped state to either the same initial state or a different third state. The first laser induced fluorescence from molecules other than the laser medium was first observed in 1966 [Yardley and Moore, 1966]. The first high-resolution measurements of transition line widths using LIF were performed in 1968 on a beam of molecular iodine with an argon ion laser [Ezekiel and Wiess, 1968]. LIF is a remarkable diagnostic technique because it can provide non-invasive, spatially localized measurements of the density and velocity distribution of a collection of absorbers/emitters. In 1973, the first LIF measurements in a plasma were used to obtain the local metastable ion velocity distribution function and relative metastable density [Stern and Johnson, 1975]. Stern and Johnson used a fixed frequency argon-ion laser and took advantage of the angles between the laser beam, viewing axis, and electric field gradients to determine the complete ion velocity distribution function. The advent of tunable dye and diode lasers is responsible for changing LIF from a cumbersome and complex process into a relatively straightforward and reliable technique that has become a common method for measuring ion and neutral distribution functions in plasmas [Hill et al., 1983]. With a tunable laser, the laser frequency is varied over a narrow frequency range containing an ion or neutral absorption transition. Typically, only metastable excited states or ground states are populated enough to use for the initial state. In a plasma, moving ions or neutrals absorb the Doppler shifted laser light, thereby pumping an electron to a higher energy, usually a short-lived, excited state. The upper state then spontaneously decays. The Doppler 
broadened absorption spectrum of the initial state is determined by measuring the intensity of the fluorescent emission as a function of laser frequency. Since the fluorescent emission is spatially localized to the laser beam and the collection optics can be designed to overlap only a small region of the beam, LIF measurements can be performed with considerable spatial resolution $\left(\sim 1 \mathrm{~mm}^{3}\right)$. The relative initial state density (with an absolutely calibrated system), ion or neutral temperature, and net drift velocity can be determined from the frequency dependence of the measured absorption spectrum.

Recent experiments have also demonstrated that LIF can be used to measure perturbations to the ion velocity distribution function in a plasma resulting from the interaction of the ions with an electrostatic wave. By fitting theoretical expressions to the measured perturbed ion velocity distribution function, the wave number of the electrostatic wave can be determined. Therefore, LIF diagnostics can also non-invasively measure the wave numbers of electrostatic plasma waves at a single spatial location [Skiff and Anderegg, 1987; Sarfaty et al., 1996]. Skiff and co-workers recently used LIF to measure electric field magnitudes with high spatial resolution in a shock wave experiment [Skiff et al., 2001] by measuring the individual terms of the Vlasov equation. In the future, the lower costs, compact size, and ease of use of tunable diode lasers will enable many more plasma research groups to employ LIF as a basic research tool [Severn et al., 1998]. The only significant difficulty with using tunable diode lasers for LIF is the small output power of tunable, visible diode lasers $(\sim 10 \mathrm{~mW})$. Recently, the WVU Helicon plasma group developed a portable, diode laser, LIF system for argon plasmas that can be taken to laboratories around the world for collaborative experiments [Boivin et al., 2002].

\subsubsection{Laser Induced Fluorescence of Argon Ions}

The Doppler shift in transition frequency for an emission or absorption line of a moving ion viewed from the laboratory frame is given by

$$
v=v_{o}\left(\frac{1-\frac{\mathrm{v}}{c} \cos \theta}{\sqrt{1-\frac{\mathrm{v}^{2}}{c^{2}}}}\right)
$$


where $v$ is the shifted frequency of the line (in the laboratory frame), $v_{o}$ is the proper frequency of the line, $c$ is the speed of light, and $v \cdot \cos \theta$ is the speed along the line of sight between the observer in the laboratory frame and the emitter/absorber. Since the physics and the mathematics are essentially the same for emission and absorption lines, the remainder of this LIF discussion will be limited to absorption lines. For nonrelativistic ions, $\mathrm{v} / \mathrm{c}$ is small and equation (3.1) becomes

$$
v=v_{o}\left(1 \mp \frac{\mathrm{v}}{c}\right)\left(1-\frac{\mathrm{v}^{2}}{2 c^{2}}\right)=v_{o}\left(1 \mp \frac{\mathrm{v}}{c} \pm \frac{\mathrm{v}^{2}}{2 c^{2}} \mp \frac{\mathrm{v}^{3}}{2 c^{3}}\right)
$$

where the \pm comes from the $\cos \theta$ term for ions moving towards $\left(\theta=0^{\circ}\right)$ or away from the observer $\left(\theta=180^{\circ}\right)$. Keeping only first order terms, the frequency shift of the absorption line is

$$
\Delta v=v-v_{o}=\mp v_{o} \frac{v}{c}
$$

for a single ion.

For a Maxwellian distribution of ion velocities,

$$
f(\mathrm{v})=A \exp \left(-m\left(\mathrm{v}-\mathrm{v}_{\mathrm{o}}\right)^{2} / 2 k_{b} T\right),
$$

where $m$ is the mass of the species, $k_{b}$ is Boltzmann's constant, $\mathrm{v}_{\mathrm{o}}$ is average drift speed of the entire distribution, and $T$ is the temperature of the ions, the Doppler broadening of an ion absorption line is given by

$$
I(v)=I_{o} \exp \left(-m\left(v-v_{o}^{*}\right)^{2} / c^{2} v_{o}^{2} 2 k_{b} T\right)
$$

where $I(v)$ is the absorbed photon flux as a function of frequency, $I_{o}$ is the maximum photon flux absorption, and $v_{o}^{*}=v_{o}+v_{o} \mathrm{v}_{\mathrm{o}} / c$ is the average proper frequency of the transition when viewed from the laboratory frame. Rather than measure the absorption profile directly, the emission intensity from the excited state is monitored as the laser frequency is varied in a typical LIF measurement. Thus, the Doppler broadened absorption line, Equation (3.5), is indirectly measured.

Doppler broadening of the absorption line due to ion motion is not the only line broadening mechanism that must be considered in a LIF measurement. Other mechanisms such as the natural line width, Stark broadening, power broadening, and Zeeman broadening will add to the broadening of the absorption line. To use LIF to 
measure the ion temperature from the Doppler broadened line shape, the contribution to the width of the line from the other broadening mechanisms must be either substantially smaller then the Doppler broadening or subtracted from the measured line shape. For the experiments described in this work, the contributions to the width of the absorption line from all other line broadening mechanisms except Zeeman broadening have been found to be much smaller than Doppler broadening [Boivin, 1999; Keiter, 1999].

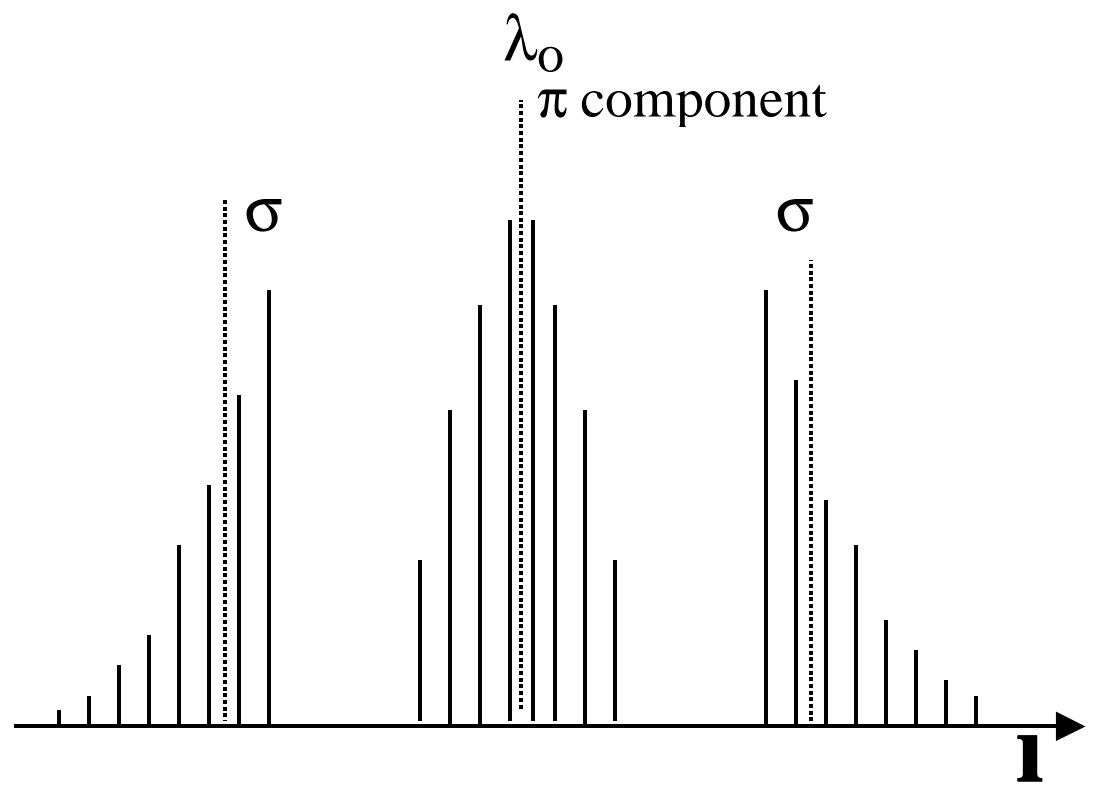

Figure 20: Schematic of the sigma, $\sigma$, and pi, $\pi$, transitions for the $611.49 \mathrm{~nm}$ argon ion absorption line. The height of each line corresponds to the statistical weighting of each transition as a function of wavelength [Boivin, 2002].

Zeeman broadening is more complicated than the other broadening mechanisms. Technically, Zeeman broadening is a splitting of quantum states due to electron spins that are either aligned or anti-aligned with the applied magnetic field. When an electron makes a transition from one orbital angular momentum state to another, the electron spin can remain the same, go from spin up to spin down, or go from spin down to spin up. To conserve angular momentum, the electron can only make a spin flip if the absorbed photon has the appropriate angular momentum. Left or right circularly polarized photons, relative to the applied magnetic field direction, have the proper angular momentum for spin changing transitions. These $\Delta m= \pm 1$ transitions, where $m$ is the spin 
angular momentum state, are called sigma transitions, $\sigma$. When the absorbed photon has no angular momentum along the magnetic field direction, the $\Delta m=0$ transitions are pumped. The $\Delta m=0$ transitions are called pi, $\pi$, transitions. Figure 20 shows an example of the Zeeman splitting of the sigma and pi lines as a function of wavelength for the $611.4923 \mathrm{~nm}$ argon ion absorption line. For thermal particle distributions, each Zeeman component is also Doppler broadened. To get a true measurement of the temperature from the measured LIF signal, the signal has to be de-convolved into the individual Zeeman components [Boivin, 2002]. However, for typical LIF measurements in HELIX using the $611.4923 \mathrm{~nm}$ absorption line, the wavelength spread of each of the two sets of sigma lines and the set of pi lines is much less than the Doppler broadening for each of the individual sigma and pi lines [Boivin, 2002]. In this case, the two sets of sigma lines can be treated as two Doppler broadened lines shifted over from the proper wavelength by the statistically weighted average Zeeman shift of the individual sigma lines. Likewise, the pi lines can be considered a single Doppler broadened line. Only for magnetic field strengths above $1200 \mathrm{G}$ and ion temperatures below $0.06 \mathrm{eV}$ is it necessary to consider the Zeeman splitting of the individual lines when calculating the ion temperature from the measured Doppler broadened absorption line.

\subsubsection{Laser Induced Fluorescence Apparatus}

For the LIF measurements used in this work, a tunable ring dye laser was directed through an argon plasma to optically pump only those ions whose Doppler shifted absorption frequency matched the laser frequency. The absorption line chosen $(611.4923$ nm) pumps the ions from an excited metastable state to a short lived excited state, $\left(3 d^{\prime}\right)^{2} G_{9 / 2} \rightarrow\left(4 p^{\prime}\right)^{2} F_{7 / 2}^{0}$. The decay of the upper state to a lower level excited state,

$\left(4 p^{\prime}\right)^{2} F_{7 / 2}^{0} \rightarrow\left(4 s^{\prime}\right)^{2} D_{5 / 2}$, is accompanied by the emission of $461.0 \mathrm{~nm}$ photons (Figure 21). The intensity of the fluorescent emission is proportional to the number of ions in the original metastable excited state. The fluorescent emission is measured as a function of laser frequency and fitted to Equation (3.5). 


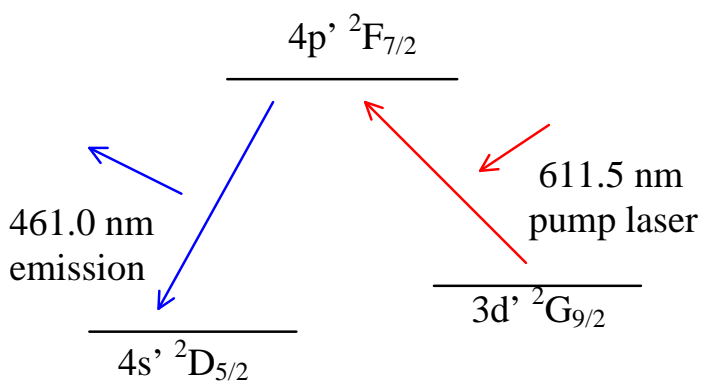

Figure 21. The argon ion LIF scheme used in these experiments. The initial state is a metastable state for singly ionized argon using the notation described in Bashkin and Stoner [1978].

The first LIF measurements of the ion velocity distribution function in HELIX took place in 1996 [Scime et al., 1998]. Since 1996, a number of other HELIX experiments have employed LIF to measure ion temperatures and ion flow velocities [Scime et al., 1998; Keiter, 1999; Kline et al., 1999; Balkey, 2000]. For the experiments reported in this work, modifications were made to the injection and collection optics on HELIX and an iodine cell was added to the laser path to provide a zero flow (rest frame) reference (Figure 22). The LIF laser system consists of a 6 W Coherent Innova 300 argon-ion laser that pumps a Coherent 899 ring dye laser. A $10 \%$ beam splitter directs a portion of the beam through an iodine cell and into a Burleigh 1500 wavemeter. Over the range of wavelengths used for argon LIF measurements, the iodine cell has a number of absorption lines [Gerstenkorn and Luc, 1979; Gerstenkorn and Luc, 1980]. The spontaneous fluorescence from the optically pumped excited states in the iodine cell is measured with a photodiode detector and recorded for each LIF measurement. Since the iodine cell is relatively cold and is at rest in the laboratory frame, the pattern of absorption lines occurs at the same laser frequency for every measurement. Therefore, the iodine cell measurements provide a zero velocity (rest frame) reference for each LIF measurement in the plasma. With the iodine cell reference, bulk ion flows on the order of $25 \mathrm{~m} / \mathrm{s}$ can be reliably measured in argon plasmas. The Burleigh 1500 wavemeter was used for setting up and tuning the dye laser. The dye laser offset (from the central frequency) frequency was controlled with a \pm 5 volt signal from a computer controlled, National Instruments AO-6 output card. As the laser frequency was swept over 10-16 $\mathrm{GHz}, 0.01$ to $0.02 \mathrm{~nm}$, the fluorescent emission from the upper metastable level was measured with a filtered photomultiplier tube detector. The filter in front of the photomultiplier has a $1 \mathrm{~nm}$ bandpass centered on the $461.0 \mathrm{~nm}$ emission line. The output 
of the dye laser was chopped at approximately $1 \mathrm{kHz}$ with a mechanical chopper. A reference signal from the chopper controller was sent to a Stanford Research SR830 lockin amplifier that monitored the photomultiplier tube signal. The lock-in amplifier extracted the fluorescence signal from the intense background emission at the same wavelength. Although the fluorescence signal was on the order of 1 part in $10^{6}$ of the background light, typical LIF measurements using the lock-in amplifier had a signal to noise ratio of at least 20:1. After the laser light passes through the mechanical chopper, it is coupled into a fiber optic cable with a fiber coupler. The fiber optic cable transported the laser light from the laser lab into the helicon lab where several different sets of injection optics were mounted on the HELIX chamber. The collected light is also transported to the photomultiplier detector by fiber optic cable. This configuration enables LIF measurements to be performed at different locations by simply moving the fiber optic cables. No other changes to the experimental hardware or data acquisition apparatus are required. The collection optics are optimized to collect the maximum amount of light through a 1" lens while still matching the numerical aperture of the lenses to the collection fiber optic cable.

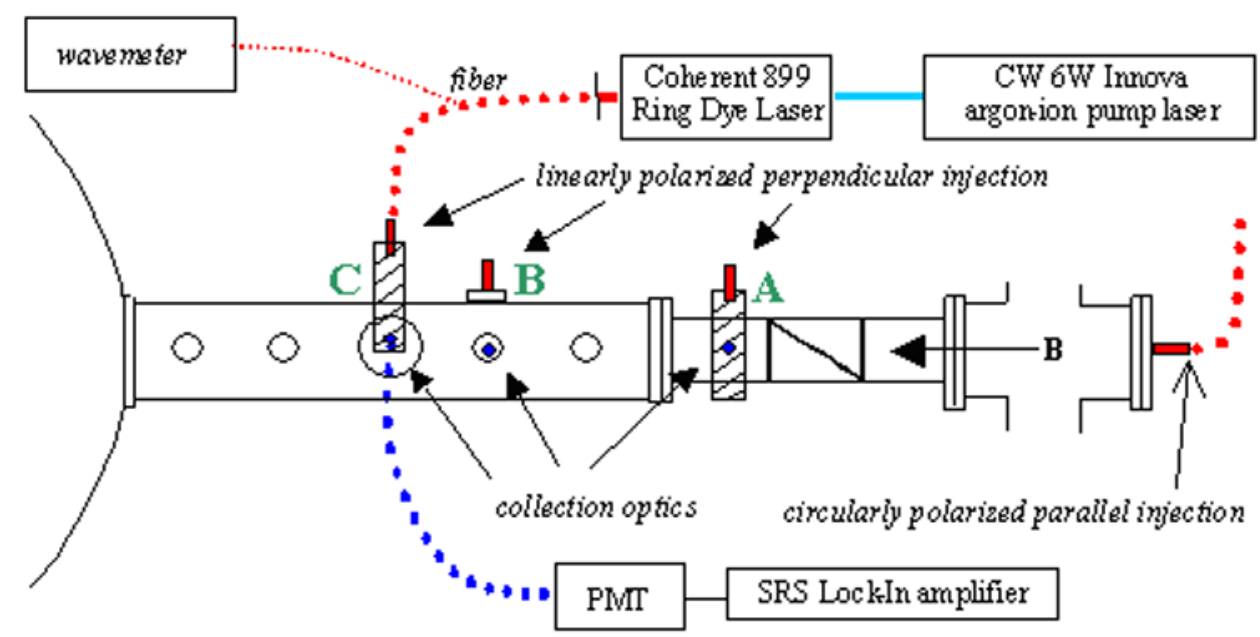

Figure 22: The experimental configuration for perpendicular and parallel LIF measurements in HELIX. The iodine cell is not shown. The letter " $\mathrm{A}$ " marks the position of the Lexan holder $5 \mathrm{~cm}$ from the antenna. "B" marks the location of the injection and collection optics $35 \mathrm{~cm}$ from the antenna and " $\mathrm{C}$ " is at the location of the two-dimensional measurement stage $65 \mathrm{~cm}$ from the antenna. 
For the first time in HELIX, LIF measurements were performed at three different axial locations. The first location was $65 \mathrm{~cm}$ from the left edge of the antenna (when the apparatus is viewed as shown in Figure 22) at the 6" crossing ports of the stainless steel section of the plasma chamber. The perpendicular injection and collection optics were mounted on two Velmex ${ }^{\mathrm{TM}}$ computer controlled, motorized stages that enabled the LIF measurement volume to be scanned through a two dimensional plane perpendicular to the axis of the plasma source (Figure 23). The optics and the horizontal motorized stage were mounted on the other motorized stage so that the injection and collection optics could be scanned vertically without altering the overlapping fields of view of the optics. The horizontal stage could then scan the collection optics along the path of the collimated laser beam. With the XY translation stage, ion temperatures, ion flow speeds, and relative metastable ion densities (assuming a uniform electron temperature profile) were measured over a $8 \mathrm{~cm} \times 8 \mathrm{~cm}$ region. The measurement plane was limited to $8 \mathrm{~cm} \times 8 \mathrm{~cm}$ by the 4" inch view ports. The overlapping injection and collection fields of view localized the measurement region to a volume of approximately $0.3 \times 0.3 \times 0.8 \mathrm{~cm}^{3}$. For measurements in the parallel direction, the injection optics were mounted on a Velmex ${ }^{\mathrm{TM}}$ motorized stage attached to the far right end of the plasma chamber (see Figure 22). By simultaneously moving the parallel injection optics at the end of the source and the collection optics on the XY stage, radial scans of the parallel ion distribution function were accomplished. In addition to the collimating and focusing lenses in the parallel injection optics, an Oriel linear polarizer-quarter wave plate combination was used to convert the unpolarized laser light exiting the fiber optic cable into circularly polarized light. Since the laser beam injected along the source axis was of a single circular polarization, only of one of the two $\sigma(\Delta m=+1)$ transitions was optically pumped. Therefore, the small internal Zeeman splitting of the $\sigma$ lines were ignored during analysis of parallel LIF measurements at magnetic field strengths under 1000 Gauss [McChesney, 1989]. 


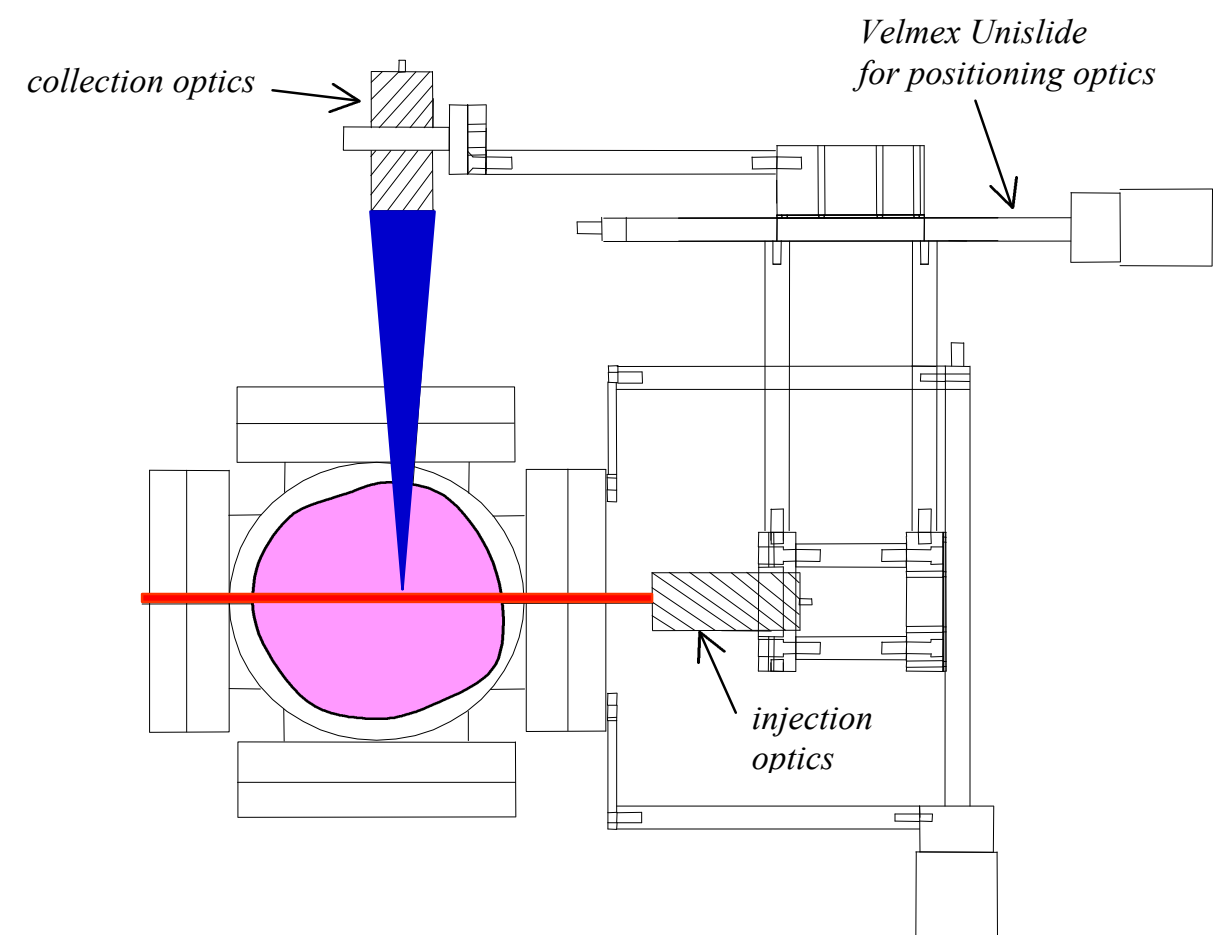

Figure 23: Schematic diagram of the two dimensional LIF apparatus located at $z=65 \mathrm{~cm}$.

The second axial location at which LIF measurements were made is $35 \mathrm{~cm}$ from the left edge of the helicon antenna. The perpendicular and parallel optics were mounted on two 2 3/4" Conflat ${ }^{\mathrm{TM}}$ view ports. For most of these experiments, the perpendicular injection and collection fields of view overlapped in the center of the plasma with a measurement volume of $0.4 \times 0.4 \times 0.6 \mathrm{~cm}^{3}$. For measurements of the perpendicular ion temperatures at different radial positions, the injection optics were tilted relative to the window normal and the collection optics kept aligned along the same chord through the plasma. The laser beam was injected through the center of the view port for each measurement and the radial location of the measurement volume determined geometrically. Since the size of the measurement volume changed for each injection angle, only the radial profiles of ion temperature and flow speed were measured using oblique injection. Measurements of the ion distribution function along the magnetic field at the $35 \mathrm{~cm}$ location were accomplished using the parallel injection optics described previously and the fixed collection optics at the $35 \mathrm{~cm}$ location. 


\section{Collection optics}

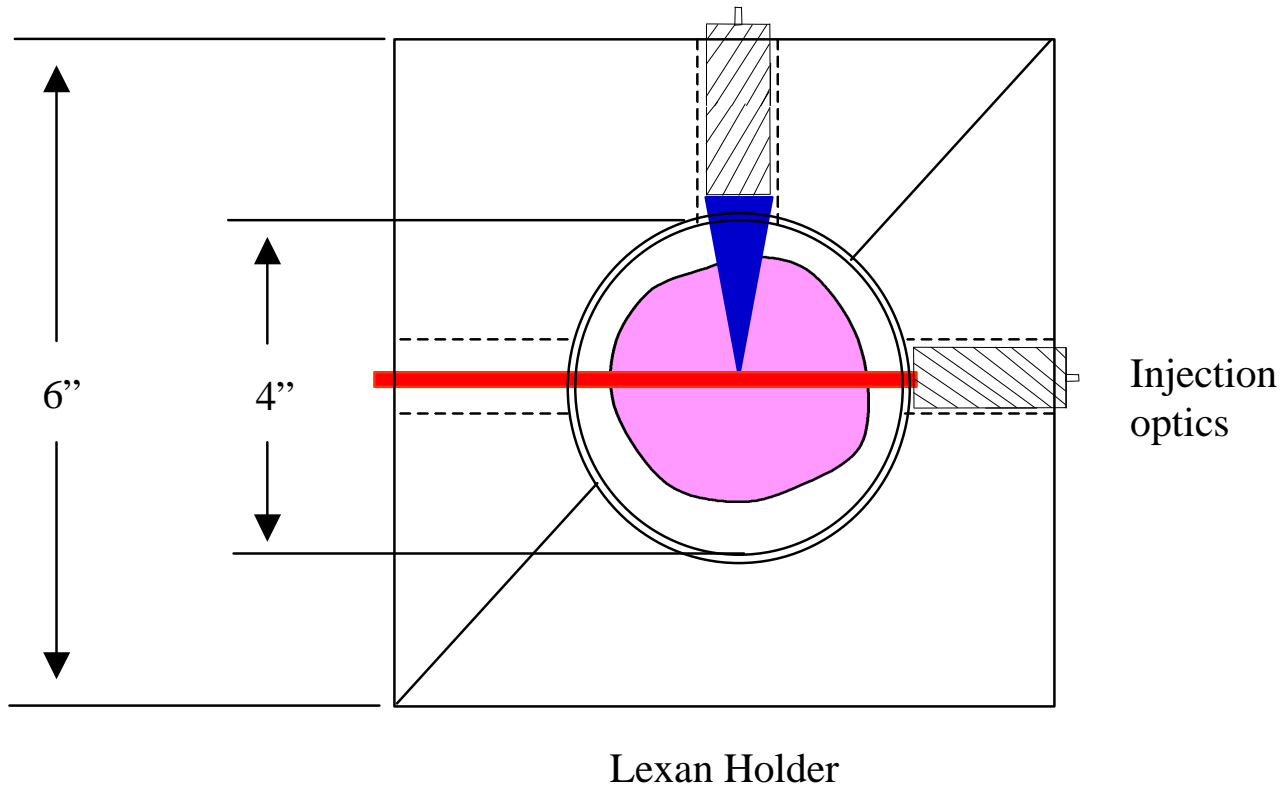

Figure 24: Lexan holder for the injection and collection optics used for LIF measurements in the Pyrex section of the vacuum system.

The third axial location for LIF measurements was $5 \mathrm{~cm}$ from the leftmost edge of the helicon antenna (Figure 22). The perpendicular injection and collection optics were mounted in a precision milled block of Lexan (Figure 24.) The Lexan fixture clamped around the Pyrex section of the chamber and could be slid along the axis of the source. For perpendicular LIF measurements, the sample volume was approximately $0.2 \times 0.2 \times$ $0.5 \mathrm{~cm}^{3}$. For parallel LIF measurements, the circularly polarized laser beam was injected from the end of the source and the collection optics mounted in the Lexan fixture focused the fluorescent emission into the collection fiber.

In addition to the iodine cell photodiode signal, the output of the SR830 lock-in amplifier, the output power level signal from the ring dye laser, and the sweep voltage sent to the dye laser controller were recorded with a 200 kilosample/s digitizer for each scan of the dye laser frequency. The digitizer was controlled through a custom LabWindows ${ }^{\mathrm{TM}}$ interface and the data stored on a PC. The output signal from the SR830 lock-in amplifier was normalized to the laser power output signal to account for variations in the laser power during the sweep of the laser frequency. Several LIF measurements were often averaged together to improve signal to noise levels. 


\subsubsection{Laser Induced Fluorescence Data Analysis}

Typical measurements of the perpendicular and parallel ion velocity distributions in a HELIX argon plasma are shown in Figure 25. The ion temperature and the center frequency of the velocity distribution are determined from a nonlinear fit to the data of Equation (3.5) for an argon plasma;

$$
I(v)=I_{o} \exp \left(-0.0779\left(v-v_{\mathrm{o}}\right)^{2} / T\right),
$$

where $T$ is the ion temperature in $\mathrm{eV}$ and $v_{\mathrm{o}}$ is the center frequency in GHz. Shifts in the center frequency of the distribution relative to the reference iodine transitions provide a measure of the absolute drift velocity of the ions.

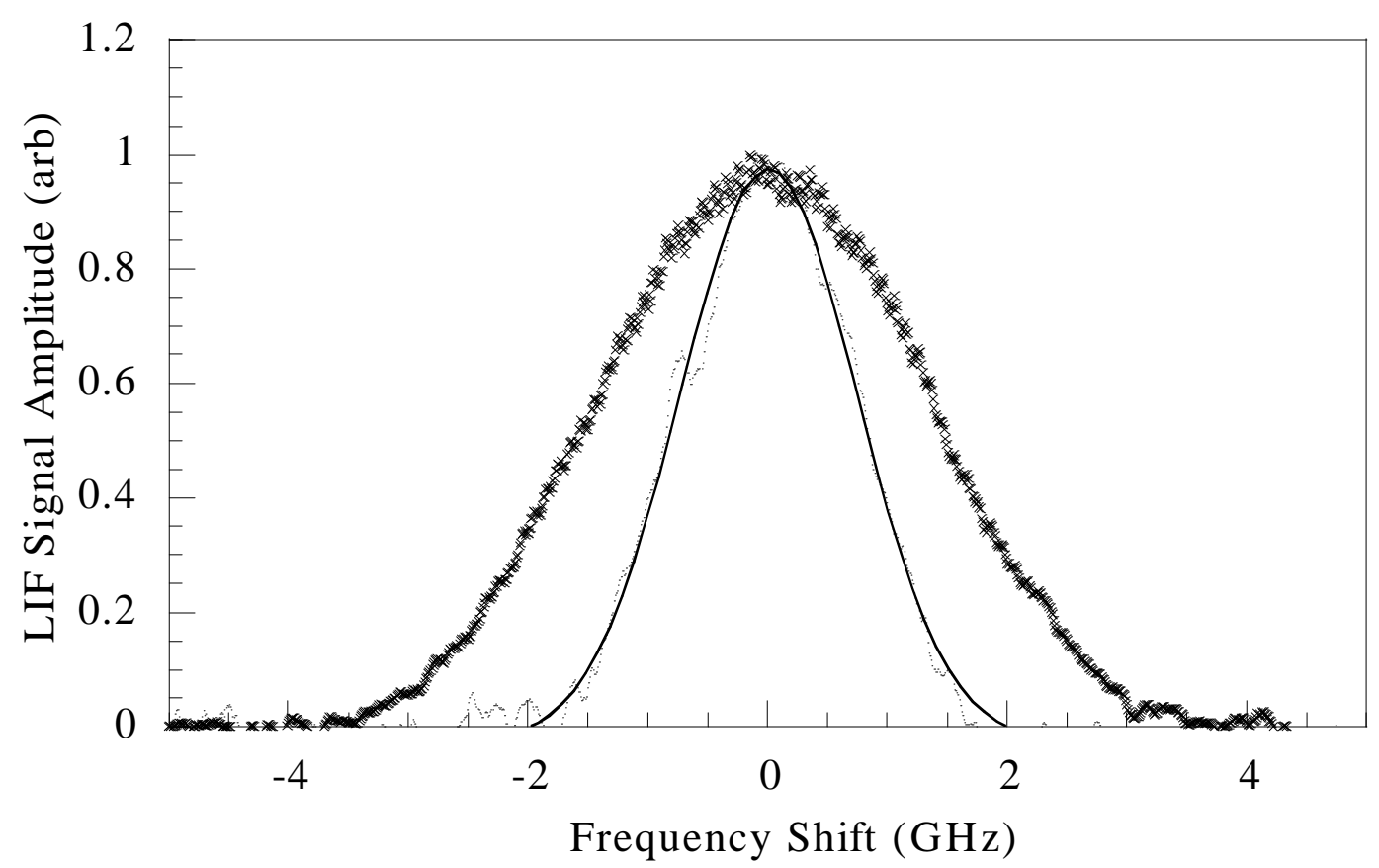

Figure 25. The parallel (dots) and perpendicular (X) argon ion velocity space distribution functions in HELIX. For this data, $T_{i \perp}=0.28 \mathrm{eV}, T_{i \|}=0.09 \mathrm{eV}$, and the fit to the parallel distribution function data is shown [Balkey, 2000].

\subsection{Measurement of Magnetic Fluctuations}

Magnetic fluctuation sense coils were used to measure the wavelength of electromagnetic waves in the plasma. The sense coils are nothing more than a series of loops of small gauge wire. A measurable voltage is induced across the ends of the windings on a coil form. When there is a time dependent change in the magnetic flux 
through the coil, a voltage is induced across the coil. With an array of sense coils, the wave vector of electromagnetic waves can be determined from measurements of the phase difference between two coils, the distance between the coils, and the direction of the vector normal to the plane of the coils.

\subsubsection{Magnetic Fluctuation Coil Theory}

The voltage induced across the ends of a magnetic fluctuation coil is given by Faraday's Law of Induction

$$
\nabla \times \vec{E}=-\frac{\partial \vec{B}}{\partial t}
$$

Assuming a sinusoidal variation of the magnetic field and integrating over the surface area enclosed by the coil, equation (3.7) becomes

$$
\int(\nabla \times \vec{E}) \cdot d a \hat{n}=-\int \omega \vec{B} \cdot d a \hat{n},
$$

where $d a$ is differential area and $n$ is a vector normal to the plane of the coil. Using Stokes' theorem Equation (3.8) becomes

$$
\oint \vec{E} \cdot d \vec{l}=-\omega \vec{B} \cdot A \hat{n}
$$

where $A$ is the total coil area and $d l$ is a differential line segment along the perimeter of the area. The integral on the left hand side of the equation is equal to the voltage induced around the perimeter of the loop. Equation (3.9) can be written as

$$
V_{\text {loop }}=\omega N B A \cos \theta,
$$

where $N$ is the number of turns in the coil and $\theta$ is the angle between the magnetic flux and the surface normal vectors. Equation (3.10) represents the frequency response of a single ideal coil to magnetic fluctuations.

There are some experimental limits on this theoretical derivation for magnetic fluctuation coils. Equation (3.10) assumes a constant magnetic field over the coil area, thereby limiting the wavelength the coils can measure. For the coils in these experiments, the coil diameter was $0.05 \mathrm{~cm}$. Assuming the wave fields are uniform over 
$1 / 10$ of the wavelength, the largest possible wave number that can be measured is on the order of $k \sim 12 \mathrm{rad} / \mathrm{cm}$. Since the self-inductance and turn-to-turn capacitance of a many turn coil can significantly affect the frequency response of the coil, the coils used in these experiments were all calibrated before use and the frequency response corrected during analysis of the data. The experimental challenge was to have enough turns to measure low frequency fluctuations without creating resonances in the coil response that distorted measurements at other frequencies.

\subsubsection{Magnetic Fluctuation Coil Apparatus}

The number of turns and the area enclosed by the coil control the sensitivity of the coil. Increasing the coil area increases sensitivity but reduces spatial resolution. Increasing the number of turns on the coils increases the sensitivity, but reduces the high frequency response because of the decrease in the response time of coils with higher inductances. An effective sense coil design must compromise between three factors, sensitivity $\left(n d^{2}\right)$, spatial resolution $(d)$, and time resolution $\left(n^{2} d\right)$, where $n$ is the number of turns and $d$ is the diameter of the coil [Lochte-Holtgreven, 1995]. Furthermore, the turn-to-turn capacitance and the signal cable capacitance generate additional resonances in the magnetic fluctuation coil system. Therefore, wide frequency range magnetic sense coils are difficult to construct.

For the experiments reported in this work, the scientific objectives required a medium frequency probe capable of measuring fluctuations from $50 \mathrm{kHz}$ to $20 \mathrm{MHz}$; frequencies just above the ion cyclotron range to frequencies just above the maximum driving frequency of HELIX. To obtain sufficient sensitivity at the lower frequencies, the coils were constructed with 175 turns of 40 -gauge wire. The inner radius of the coils is 0.05 " and the outer radius is 0.20 ". The length of the coil form is 0.05 ". Five such coils were placed inside a boron nitride housing with the surface normal vectors of the coils all facing the same direction (Figure 26 and Figure 27). 


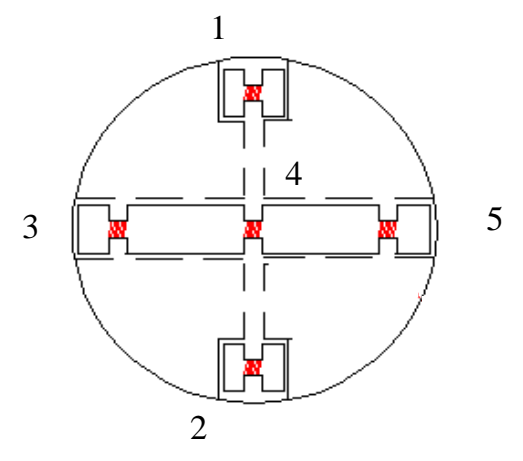

Figure 26: End view schematic of the magnetic fluctuation probe. The axes of all five coils are in the plane of the page. Each coil contains 175 turns of 40 gauge, enamel coated copper wire.

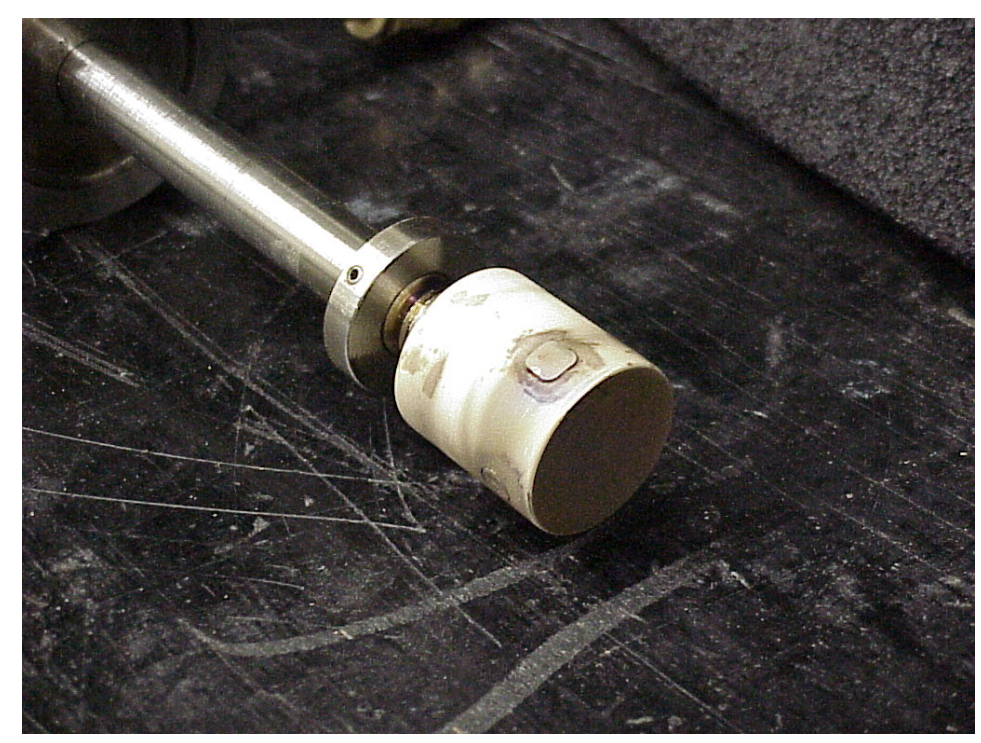

Figure 27: Photograph of the assembled magnetic fluctuation probe.

With the axes of the coils aligned with the axis of HELIX, the probe could measure the amplitude and phase of magnetic field fluctuations along the $z$ direction as a function of $z$ and $y$, i.e. perpendicular and parallel to the background magnetic field. With a $90^{\circ}$ rotation, the probe could also measure the amplitude and phase of magnetic field fluctuations along the $\theta$ direction as a function of $z$ and $\theta$, i.e. perpendicular and parallel to the background magnetic field.

The two wires from each magnetic fluctuation sense coil were connected to a center tapped transformer, Mini-Circuits TT1-6, with a frequency range of $0.004-500 \mathrm{MHz}$ [Balkey, 2000]. The center tap on the magnetic fluctuation coil side of the transformer 
was connected to ground. The center tap transformer removed electrostatic or capacitive signals from the magnetic fluctuation probe that could be mistaken for electromagnetic signals [Loewenhardt et al., 1993; Light et al., 1995]. These common mode signals occur when changes in the net charge near the magnetic fluctuation coil or signal cables induce an image current on both magnetic fluctuation coils signal wires. The large common mode rejection of the center-tapped transformer eliminated the common mode signals and coupled the inductive signal into a single, ground referenced, high frequency transmission line. The transmission line, an RG-8 coaxial cable with the shield grounded, carried the sense coil signal from the vacuum feedthrough to a Tektronics TDS430 digital oscilloscope. RG-8 cable was used instead of RG58 cable because of its smaller attenuation per unit length at frequencies above $10 \mathrm{MHz}$ [Balkey, 2000]. The Tektronics TDS430 oscilloscope could simultaneously acquire two 8192 point, fluctuation coil signal records at digitization rates up to 100 Megasamples/s.

After construction of the magnetic fluctuation probe, the amplitude and phase response of all five coils as a function of signal frequency were measured using a known fluctuating magnetic field signal and the same cabling used during the measurements. Although each of the coils was constructed the same way, the frequency dependent amplitude and phase responses were not identical. For calibration, a magnetic fluctuation coil is typically placed into a simple solenoid with a known magnetic field strength oscillating at a known frequency. However, since the configuration of the magnetic fluctuation probe used in these experiments has the axis of the magnetic field coils perpendicular to the probe shaft, a pair of coils (Helmholtz coils) was used to create the calibration magnetic field

To obtain a spatially uniform field for calibration, the diameter of the coils must be comparable to the spacing between them. The Helmholtz coils were wound on a 3" diameter, 6" long section of PVC pipe and a hole large enough for the magnetic fluctuation probe head was drilled through the side of the pipe. For large Helmholtz coils, it is difficult to couple sufficient input power at high frequencies into the coils to generate magnetic fields large enough for calibration purposes. To drive the necessary current through the Helmholtz coils, a Wavetek 0 - $50 \mathrm{MHz}$ function generator was connected to a ENI $0.3-50 \mathrm{MHz}$ linear amplifier with the output of the amplifier 
connected to the Helmholtz coils. The magnetic field strength as a function of DC current through the Helmholtz coils was measured with a calibrated Hall probe and the same magnetic field to current ratio was used to convert measurements of the AC current in the coil into magnetic field strengths during calibration. A LabWindows $\mathrm{C}$ code was written to sweep the frequency of the Wavetek function generator through a General Purpose Interface Bus (GPIB) while both the current in the Helmholtz coils (measured across a $10 \mathrm{Ohm}$ shunt resistor) and the signal from a single magnetic fluctuation sense coil were recorded by Tektronix TKS641 1 Gigasample/s digitizer.

At each frequency step, the relative phase between the sense coil voltage and the Helmholtz coil current was calculated from the Fourier space cross power spectrum (explained in the next section) between the two signals. The frequency dependent effective area, $A(\omega)$, of each coil is related to the measured voltage amplitude, $V$, and the amplitude of calibration magnetic field by $A(\omega)=V /(\omega|B|)$, where $\omega$ is the oscillation frequency. The effective area includes the physical area of the coil and any inductance effects. Calibration data for each of the five coils used in the magnetic fluctuation probe are shown in Figure 28 and Figure 29.

a)

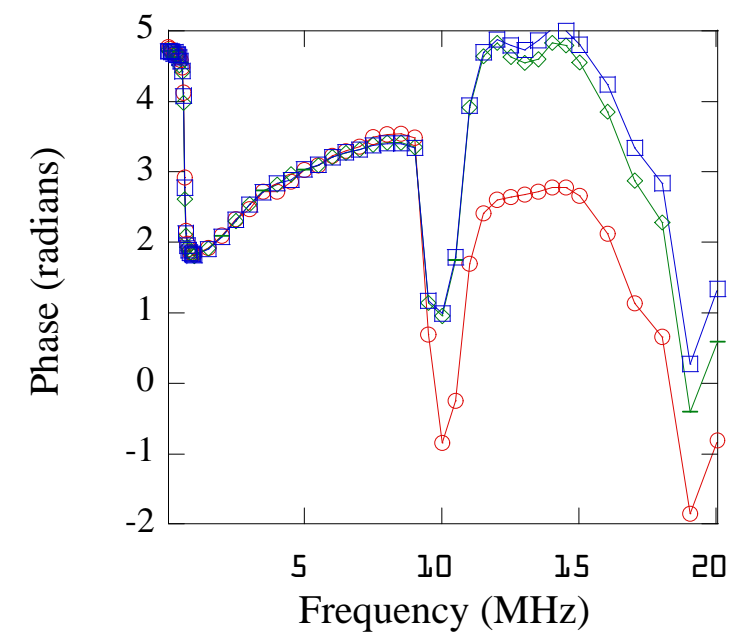

b)

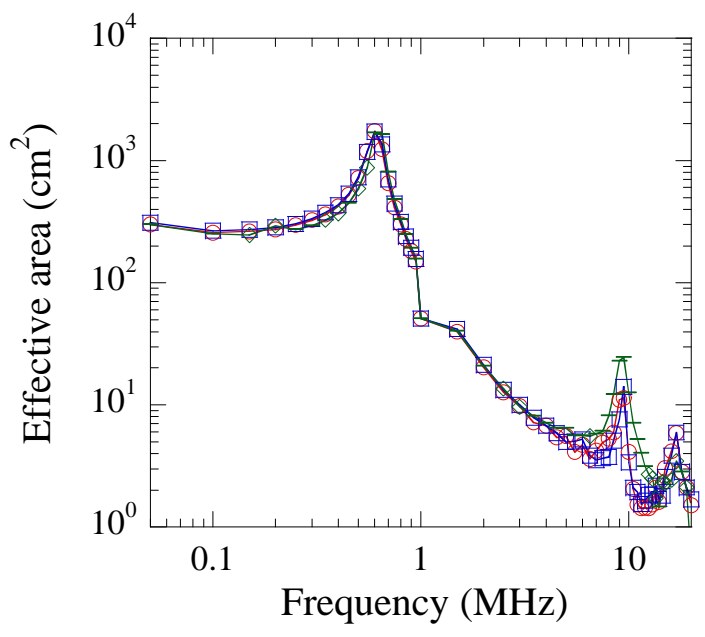

Figure 28: a) Phase calibration data for coils $(\bigcirc)$ 1, ( $\square$ ) 2, and $(\diamond) 4$ b) Amplitude calibration data for coils $(\bigcirc) 1,(\square) 2$,and $(\diamond) 4$. Note frequency axes are on different scales for clarity. 
a)

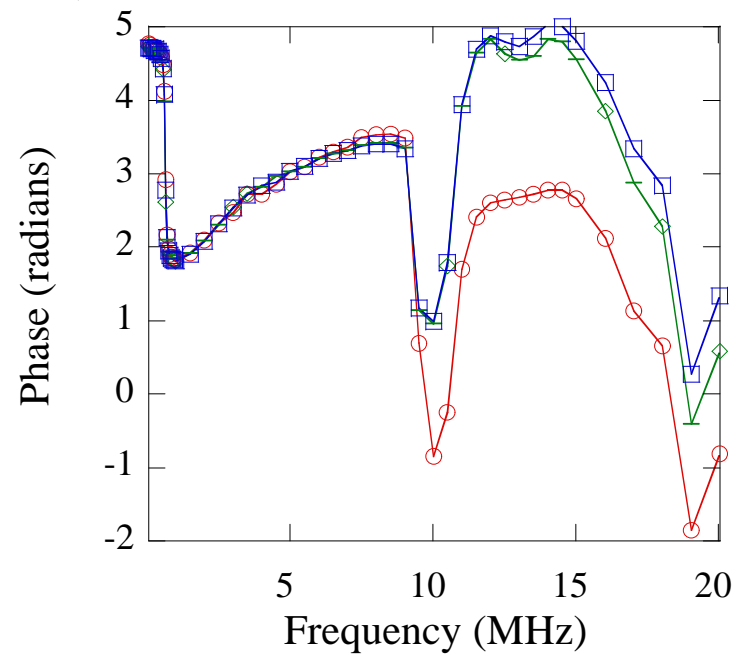

b)

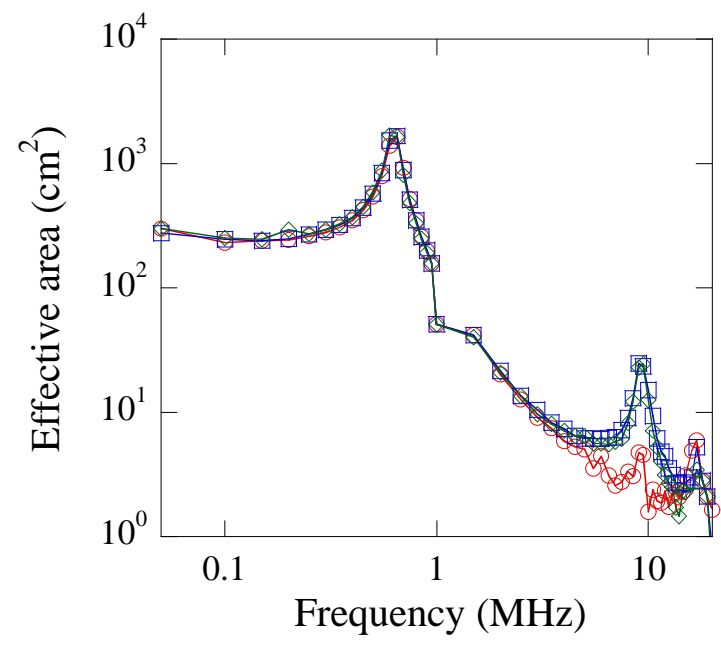

Figure 29: a) Phase calibration data for coils $(\bigcirc)$ 3, ( $\square$ ) 5, and $(\diamond) 4$ b) Amplitude calibration data for coils $(\bigcirc) 3,(\square) 5$,and $(\diamond) 4$. Note frequency axes are on different scales for clarity.

The coil areas are clearly not smooth functions of frequency, i.e. significant resonant features are apparent in the data; evidence of the difficulty in building wide band magnetic fluctuation sense coils. The original intention was to construct a piecewise spline fit to the calibration data and then use the fits to correct sense coil data after the time series was Fourier transformed into frequency space. After the corrections were applied, the modified frequency space data would be inverse Fourier transformed to construct a complete time series record corrected for frequency dependent phase and amplitude effects [Balkey, 2000]. The complex resonant features in the calibration data, however, are not amenable to simple fitting algorithms and the details of the calibration data near the resonances could not be used. To avoid artificially introducing phase shifts at particular frequencies, only data recorded using sense coil pairs 1 and 2 and 4 and 5 were used to determine wave numbers in these experiments. For these particular pairs of sense coils, the differences in phase and amplitude are small over the range of frequencies investigated (Figure 30). For those few frequencies where significant differences in phase were measured, the intrinsic phase differences were subtracted during the wave number calculations. 
a)

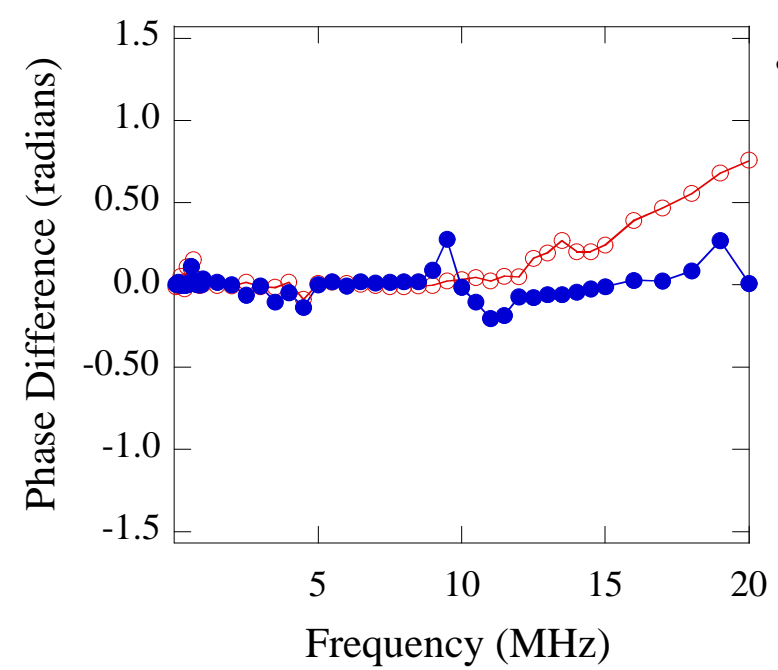

b)

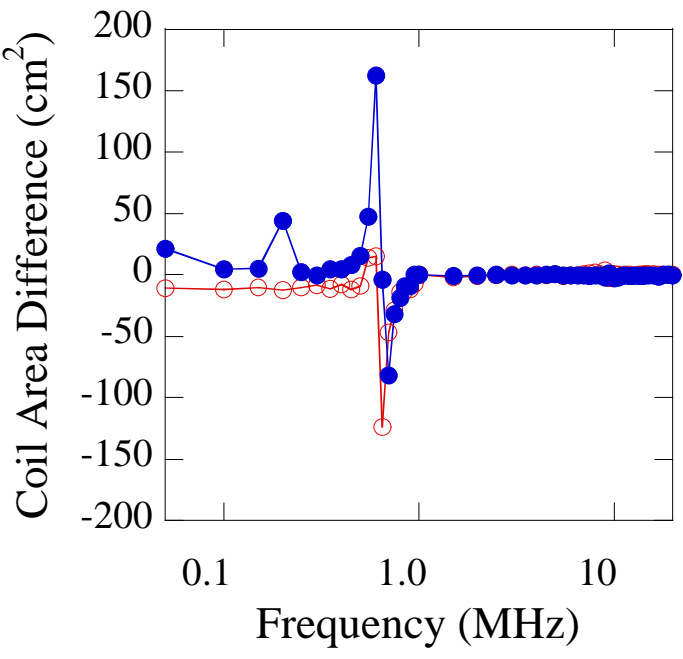

Figure 30: a) Phase difference between coils (•) $1 \& 2$ and (O) $4 \& 5$ b) Amplitude difference between coils $(\bullet) 1 \& 2$ and $(\bigcirc) 4 \& 5$. Note frequency axes are on different scales for clarity.

\subsubsection{Wave Number Analysis for Fixed Probe Pairs: Magnetic Fluctuation Coils and Electrostatic Probe}

At a specific instant in time, the phase difference, $\theta$, between signals from two spatially separated sense coils results from the spatial portion of the traveling wave phase, $(\omega t-\boldsymbol{k} \cdot r)$, where $\theta=\boldsymbol{k} \cdot \boldsymbol{r}$. For a measured phase difference and a known coil separation distance, $|\mathbf{r}|$, the wave number, $k$, can be determined from the ratio $\theta / r$. The critical step in the wave number calculation is the determination of the phase difference between the two signals.

For ideal sinusoidal signals, the phase difference can be measured directly from time series measurements of the two signals. However, typical signals are distorted by harmonics of the wave and noise. When the signal to noise ratios of the individual signals are large, heterodyning or filtering techniques can be employed. Such techniques require considerable electronic expertise and care must be taken to eliminate any phase differences resulting from the electronics. Heterodyning and filtering methods also have the disadvantage of being tied to a specific frequency, i.e., investigation of phase differences at a variety of frequencies requires construction of tuned electronics for each frequency of interest. Another approach to calculating the phase difference is to calculate the cross-power spectrum of the two time series. The cross-power spectrum is the Fast 
Fourier Transform (FFT) of one time series multiplied by the complex conjugate of the FFT of the second time series. From the cross-power spectrum, phase differences between the two time series are calculated in frequency space. Phase differences, and consequently wave numbers, are calculated for each frequency element of the FFTs. For signals with frequencies that do not vary in time and have large signal to noise ratios, the phase differences can be easily calculated from a single set of time series measurements. However, for signals that have a low signal to noise ratio or have frequencies and amplitudes that do vary in time, e.g., turbulent plasmas, an ensemble average of many cross-power spectra is required to extract meaningful phase difference measurements from the data [Bendat and Piersol, 1980; Beall et al., 1982; Assadi, 1994].

Before presenting the details of spectral density function (the ensemble average cross power spectra) calculations, it is helpful to first review the basic principles of a simple cross-power spectrum. The FFT's of two time series, $f_{1}(t)$ and $f_{2}(t)$,

$$
\Phi_{1}(x, \omega)=\int_{-\infty}^{\infty} f_{1}(x, t) \cos (\omega t) d t-i \int_{-\infty}^{\infty} f_{1}(x, t) \sin (\omega t) d t
$$

and

$$
\Phi_{2}(x, \omega)=\int_{-\infty}^{\infty} f_{2}(x, t) \cos (\omega t) d t-i \int_{-\infty}^{\infty} f_{2}(x, t) \sin (\omega t) d t,
$$

are multiplied times each other to create the cross-power spectrum, $P_{12}(\Delta x, \omega)$,

$$
P_{12}(\Delta x, \omega)=\Phi_{1}\left(x_{1}, \omega\right) \Phi_{2}^{*}\left(x_{2}, \omega\right),
$$

where $\Delta x$ is the spatial separation between the two measurement locations. Writing the cross-power spectrum in real and imaginary components,

$$
P_{12}(\Delta x, \omega)=\left(\varphi_{1 \mathrm{Re}} \varphi_{2 \mathrm{Re}}+\varphi_{2 \operatorname{Im}} \varphi_{1 \mathrm{Im}}\right)+i\left(\varphi_{2 \mathrm{Re}} \varphi_{1 \mathrm{Im}}-\varphi_{2 \mathrm{Im}} \varphi_{1 \mathrm{Re}}\right)
$$

the phase of the complex cross-power spectrum, an important quantity that will be used later in this discussion, becomes

$$
\Theta(\omega)=\tan ^{-1}\left(\frac{\left(\varphi_{2 \mathrm{Re}} \varphi_{1 \mathrm{Im}}-\varphi_{2 \mathrm{Im}} \varphi_{1 \mathrm{Re}}\right)}{\left(\varphi_{1 \mathrm{Re}} \varphi_{2 \mathrm{Re}}+\varphi_{2 \mathrm{Im}} \varphi_{1 \mathrm{II}}\right)}\right)
$$


Note that the phase of the cross-power spectrum depends explicitly on frequency. Although Equations (3.7) and (3.8) are mathematically accurate, the physical meaning of the phase of the cross-power spectrum is not immediately obvious.

A more intuitive approach is to write the FFT's as vectors in the complex plane

$$
\vec{\Phi}_{1}(x, \omega)=\varphi_{1 \mathrm{Re}} \hat{x}+\varphi_{1 \mathrm{Im}} \hat{y} .
$$

Equation (3.14) becomes

$$
P_{12}(\Delta x, \omega)=\vec{\Phi}_{1} \bullet \vec{\Phi}_{2}+i \hat{z} \bullet\left(\vec{\Phi}_{1} \times \vec{\Phi}_{2}\right),
$$

where $\mathbf{z}$ is a vector perpendicular to both vectors $\vec{\Phi}_{1}$ and $\vec{\Phi}_{2}$. According to Equation (3.17), the real part of the cross-power spectrum is the component of the first FFT along the direction of the second FFT, i.e. the portion of the first times series signal completely in phase with the second time series. The imaginary part of the cross-power spectrum is the component of the first time series completely out of phase with the second time series. The phase angle of the cross-power spectrum is then just the angle between the FFT vectors in the complex plane.

The spectral density function, $S(\omega, \boldsymbol{k})$, effectively an ensemble average of many crosspower spectra measurements, is a function of two variables; the angular frequency, $\omega$, and the phase between the signals (the wave number), $k$ [Assadi, 1994]:

$$
S(n \Delta k, l \Delta \omega)=\frac{1}{M} \sum_{j=1}^{M} I_{n \Delta k}\left[k^{(j)}(l \Delta \omega)\right]\left(\frac{1}{2}\left(P_{1}^{(j)}(l \Delta \omega)+P_{2}^{(j)}(l \Delta \omega)\right),\right.
$$

where $\Delta k$ and $\Delta \omega$ are the wave number and frequency bin sizes, $l$ is the number of frequency increments, $n$ is the number of wave number increments, $M$ is the number of time series sets or ensembles, $P_{l}(l \Delta \omega)$ and $P_{2}(l \Delta \omega)$ are the power spectra of each time series, and the selection function is defined as [Assadi, 1994]

$$
I_{n \Delta k}\left[k^{(j)}(l \Delta \omega)\right]=\left[\begin{array}{ll}
1 & (n-1 / 2) \Delta k<k^{(j)}(l \Delta \omega)<(n+1 / 2) \Delta k \\
0 & \text { elsewhere }
\end{array}\right] .
$$

Essentially, the spectral density function is the ensemble averaged spectral power from the individual FFTs, $\left.1 / 2 P_{l}(l \Delta \omega)+P_{2}(l \Delta \omega)\right) / M$, for each frequency from 1 to $l$ placed into 
the proper wave number (or phase) bin between 1 and $n$ using the selection function, Equation (3.19). The result is a two dimensional matrix, $S=X_{l n}$, where the individual elements are the ensemble averaged spectral power for all measured frequencies and wave numbers. By ensemble averaging, reoccurring signals at particular frequencies and phase differences (wave numbers) are enhanced and random fluctuations, i.e., noise, average to zero.

Although the spectral density approach requires a large ensemble of measurements, it has the additional advantage of providing a measure of the spectral width of turbulent fluctuations in the plasma. For a single pair of time series measurements, the crosspower spectrum will yield a unique wave number for each frequency step. In a turbulent system, many different wave numbers may exist at the same oscillation frequency. By averaging many time series together, measurements of the spectral width in wave number for a given frequency and the spectral width in frequency for a given wave number are obtained. To illustrate the analysis technique, an example is provided.

The natural $\log$ of the spectral density matrix for experimental data collected with the electrostatic probe (to be discussed later) is shown in Figure 31 at two different angular positions. Figure 31a shows a plot of the spectral density function for the electrostatic probe oriented along the applied magnetic field in HELIX at $8 \mathrm{MHz}$ and 845 Gauss. Figure $31 \mathrm{~b}$ shows a spectral density plot for the same parameters but with the electrostatic probe at a $30^{\circ}$ angle with respect to the applied magnetic field. A comparison of the two plots shows that, as expected, the measured wave numbers are different for the two cases. Perhaps the most important feature in the data is that the wave numbers change with frequency; demonstrating the dispersive nature of the plasma medium. For large periodic signals with a high signal to noise ratio, the wave numbers can be read directly from a plot of the spectral density (Figure 32). In the case of a turbulent wave spectrum, such as the one shown in Figure 33, more sophisticated data analysis is required. 


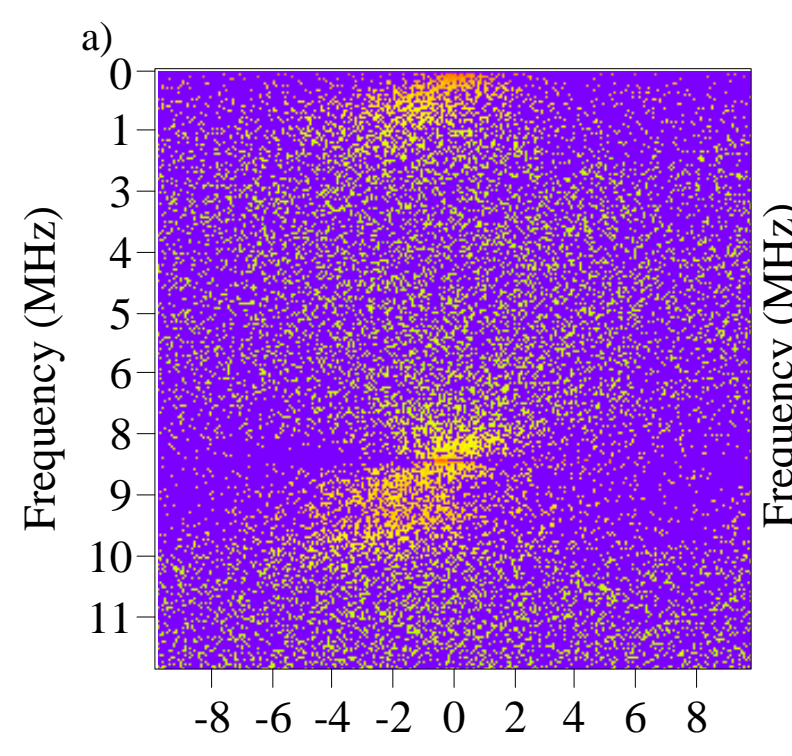

Wave Number $(\mathrm{rad} / \mathrm{cm})$

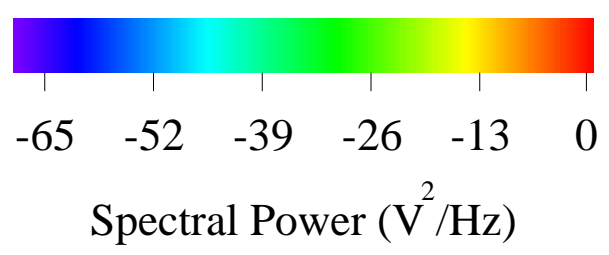

b)
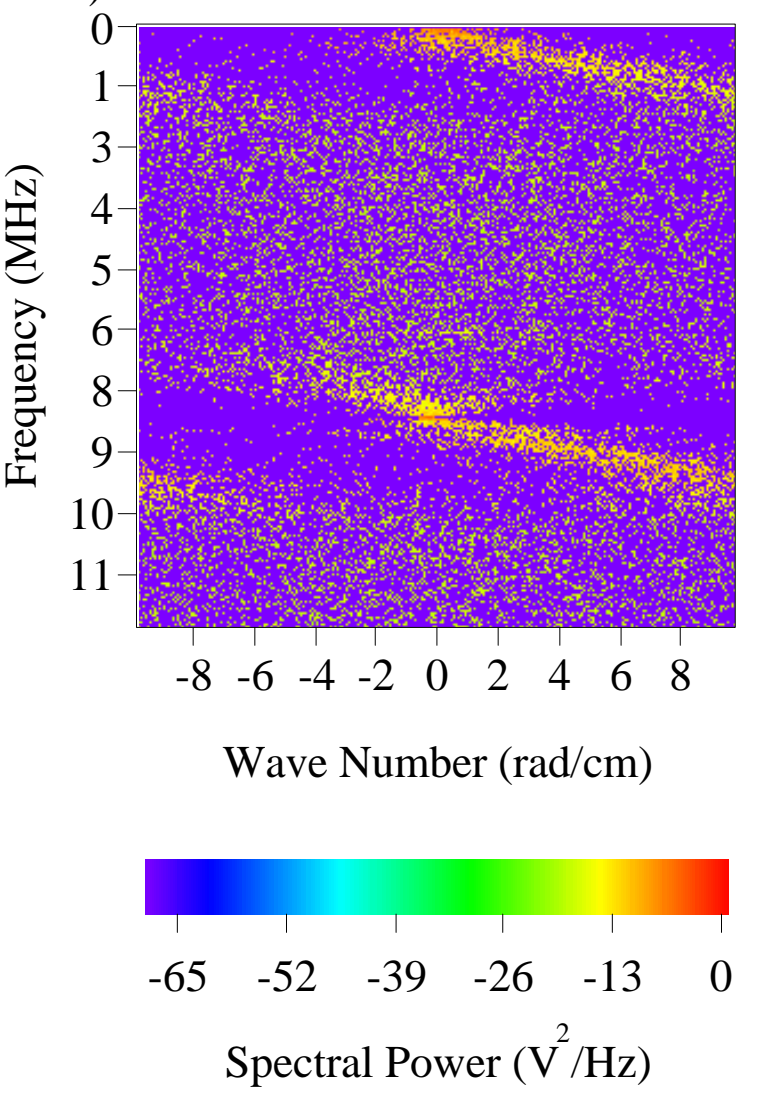

Figure 31: The spectral density versus frequency and wave number at an RF frequency of $8 \mathrm{MHz}$ and a magnetic field strength of 845 Gauss from the electrostatic probe aligned (a) along the magnetic field and (b) at a $30^{\circ}$ angle with respect to the magnetic field.

From Figure 33, it is clear that the wave number for a wave measured at exactly $8.8 \mathrm{MHz}$ is on the order of $-1.0 \mathrm{rad} / \mathrm{cm}$, but since there are so many peaks, it is difficult to determine the exact wave number value. Because, as indicated by the black lines in Figure 34, the wave has a finite frequency width, a more accurate determination of the wave number at $8.8 \mathrm{MHz}$ is possible by summing the wave number amplitudes over a band of frequencies centered at $8.8 \mathrm{MHz}$. Figure 35a shows such a sum of wave number amplitudes centered on the same frequency as shown in Figure 33. Figure 35b shows a similar sum of wave number amplitudes for electrostatic probe aligned $30^{\circ}$ relative to the magnetic field. A Gaussian curve can be fit to the data of Figure 35 to determine the peak wave number in both cases. Mathematically the wave number resolution, $\Delta \boldsymbol{k}$, using the spectral density technique is equal to $2 \pi$ times the separation distance $(d)$ divided by 
the number of points in the record $\left(R_{\text {length }}\right), \Delta \boldsymbol{k}=2 \pi d / R_{\text {length }}$. However, the real spatial resolution is limited by the size of the coil and noise in the phase measurement.

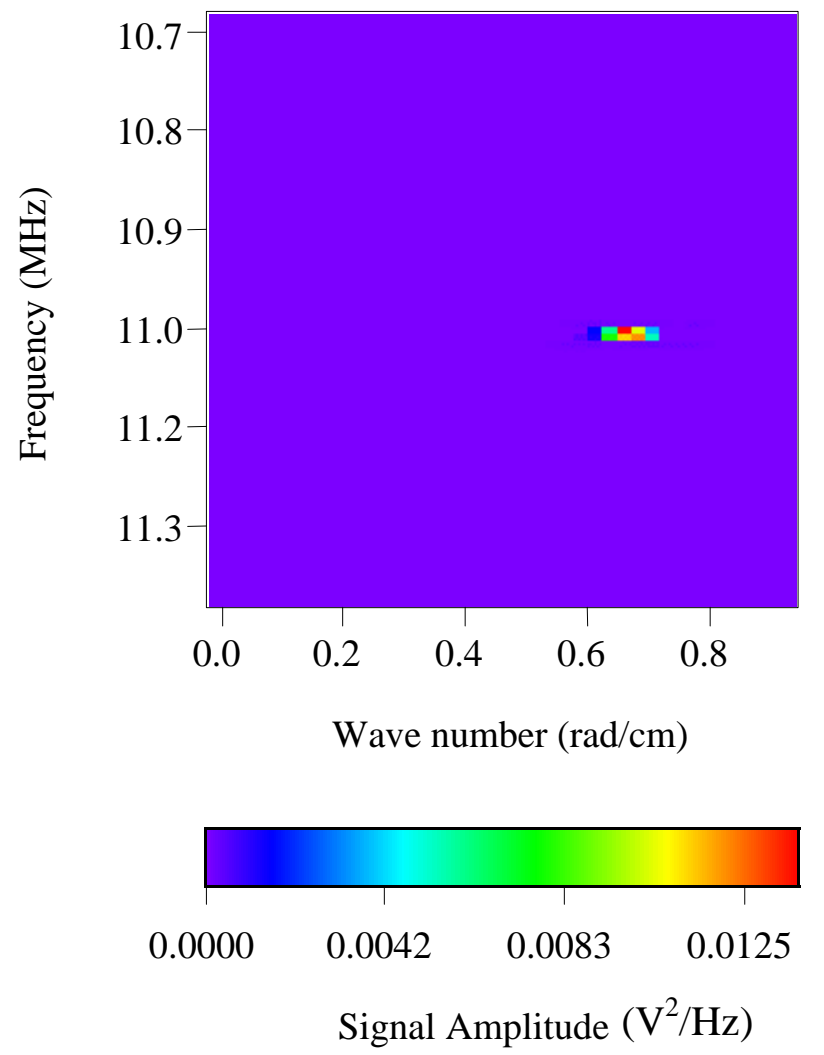

Figure 32: The spectral density versus frequency and wave number from an electromagnetic probe with a high signal to noise ratio for a RF driving frequency of $11 \mathrm{MHz}$ and magnetic field strength of 845 Gauss.

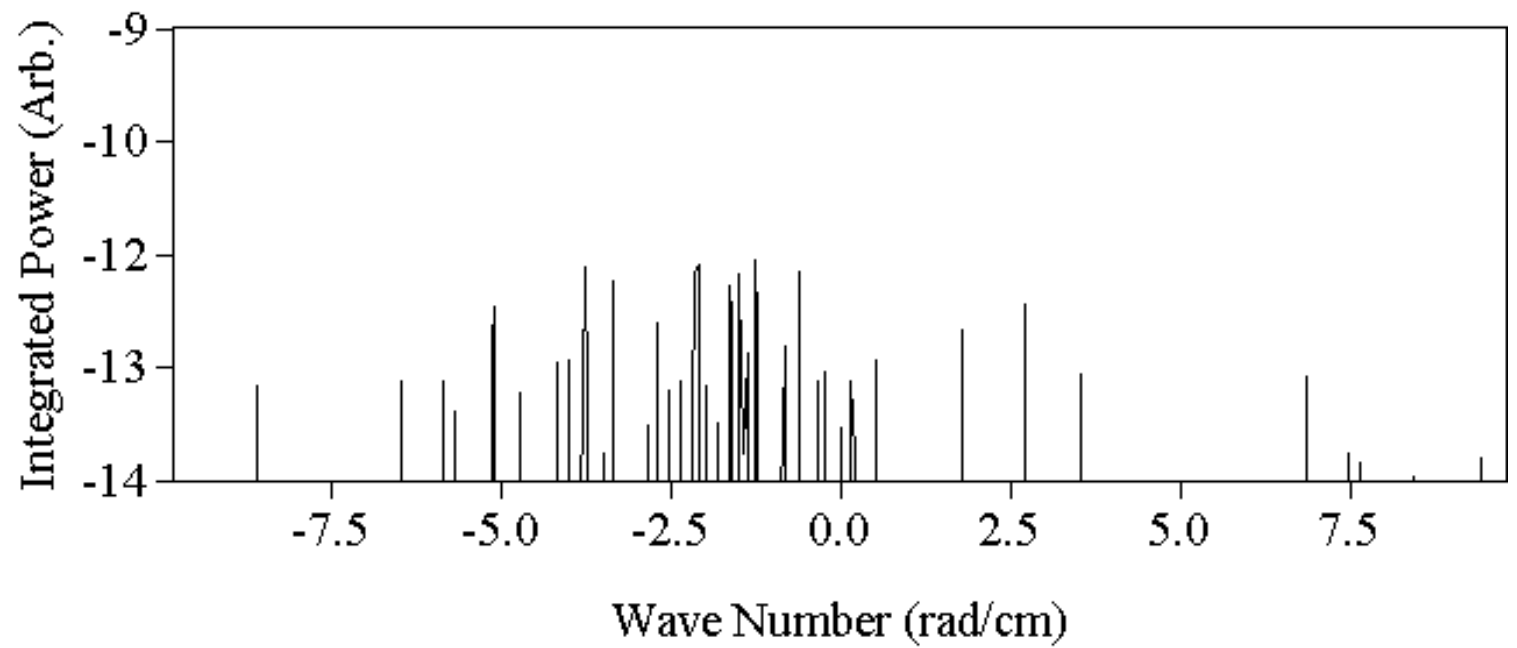

Figure 33: Wave number spectrum at exactly $8.8 \mathrm{MHz}$ from Figure 31a 


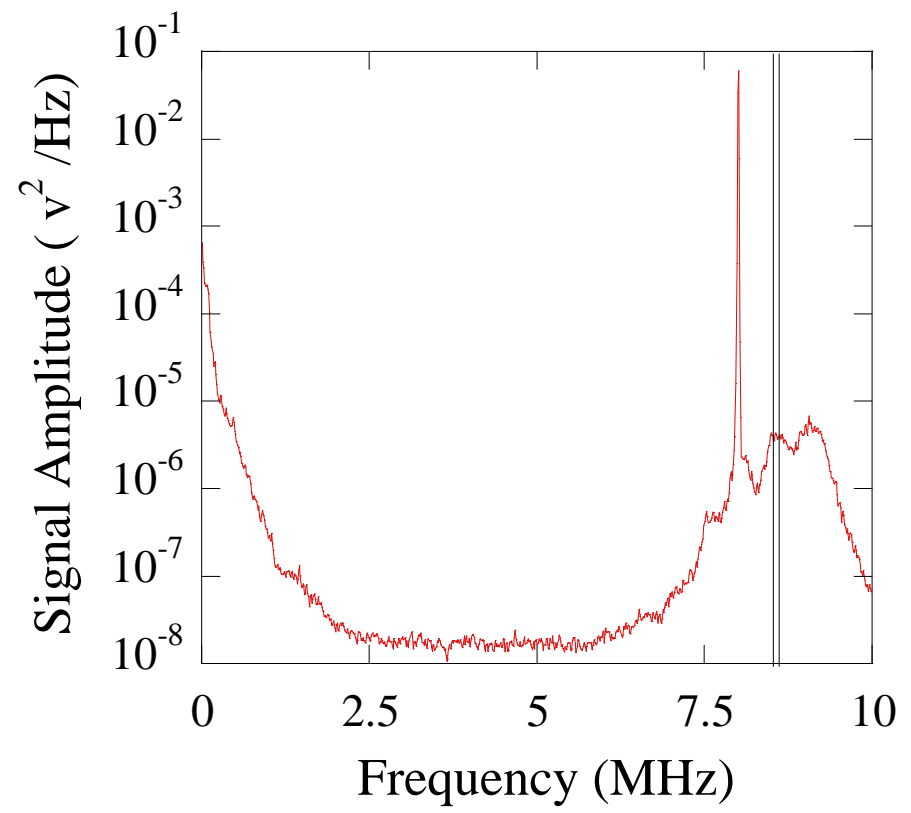

Figure 34: Frequency spectrum integrated over wave number for the spectral density data shown in Figure $31 \mathrm{a}$.

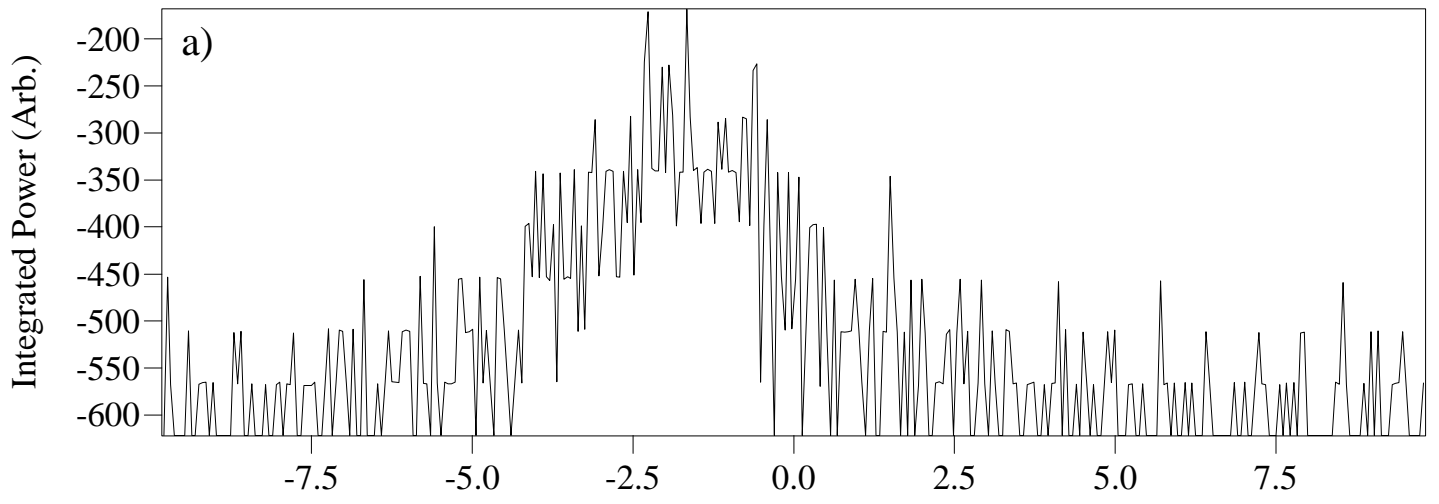

Wave Number $(\mathrm{rad} / \mathrm{cm})$

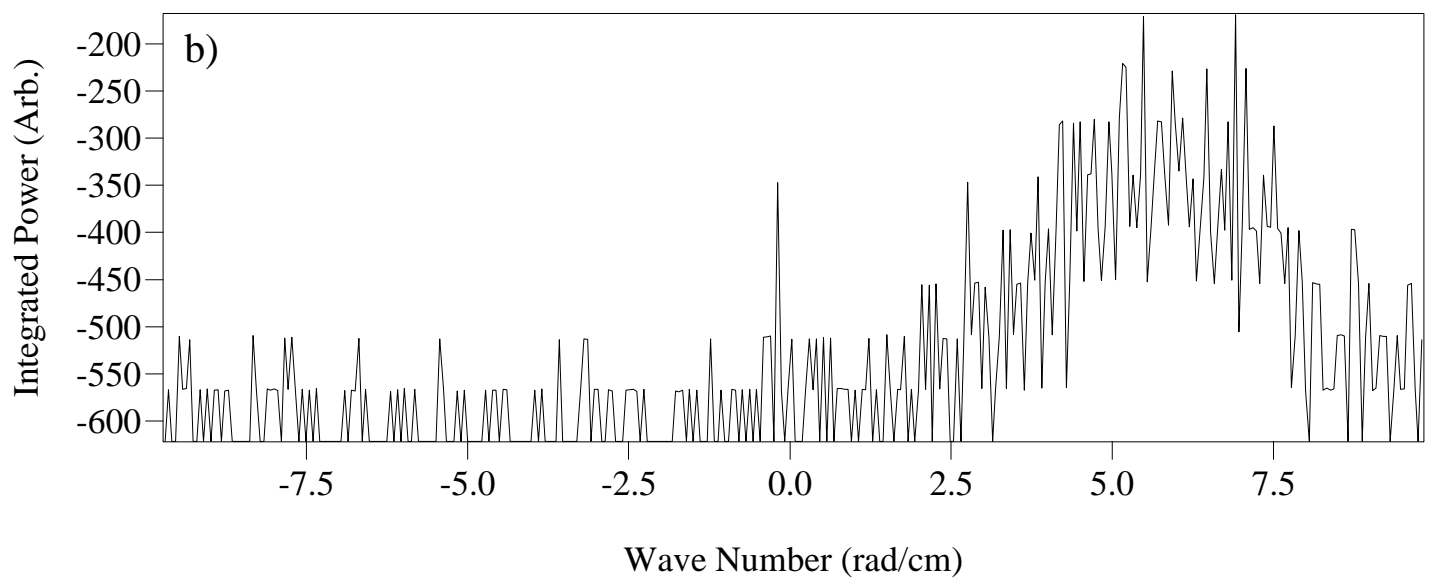

Figure 35: Wave number spectra summed over a frequency window of $70 \mathrm{kHz}$ around $8.8 \mathrm{MHz}$ for the electrostatic probe aligned in the (a) parallel direction and (b) at an angle of $30^{\circ}$. 
Two difficulties associated with using the cross-power spectrum technique with pairs of spatially fixed probes are the large amounts of data required for each measurement and spatial aliasing. In power spectrum measurements at a single spatial location, aliasing occurs when a periodic signal's frequency is larger than the Nyquist frequency $\left(f_{\text {Nyquist }}=1 / 2\right.$ sample rate $)$ of the data acquisition apparatus. In an FFT of an aliased time series measurement, the periodic signal spectral power will appear downshifted in frequency. Additional spectral peaks at harmonics of the artificially downshifted frequency are also likely to appear in the FFT. For wave number measurements using fixed probe pairs, spatial aliasing occurs when the wavelength becomes smaller than the separation distance between the two tips (see Figure 36). For the situation depicted in Figure 36, the measured phase differences are the same, but the wavelengths are not. The probe separation places a lower bound on the wavelength $(\lambda)$ of waves that can be resolved equal to the coil spacing $(d), \lambda_{\min }=d / 2$.

There are experimental procedures for determining if the signal is aliased. Aliasing in frequency space can be detected by changing the digitization rate; aliased spectral peaks will shift in frequency. Spatial aliasing can be detected by changing the separation between the probes; spectral peaks will shift in phase (wave number). Since fixed probe pairs are used for these experiments, the probe tip spacing cannot be changed. However, the probes were rotated, thereby changing the effective separation distance in the perpendicular and parallel directions with respect to the applied magnetic field. If the wave numbers are spatially aliased, different perpendicular and parallel wave numbers at different probe angles would be measured. No aliasing was detected for the waves measured in these experiments.
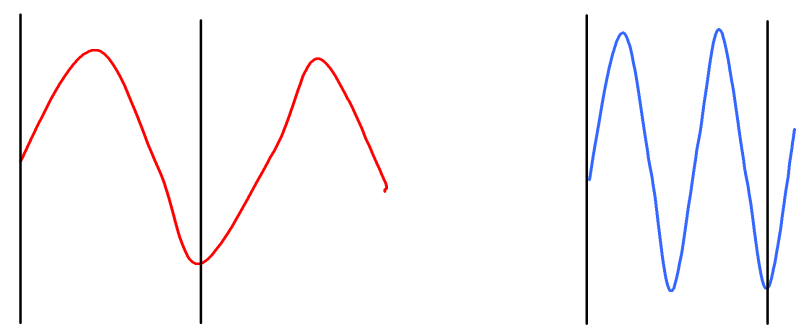

Figure 36: Illustration of spatial aliasing for fixed probe pairs. The black lines represent the probe locations and the colored lines the wave amplitudes at a given instant of time. 


\subsection{Measurements of Electron Temperature and Plasma Density (Langmuir Probes)}

The Langmuir probe is one of the most basic plasma diagnostics. It is essentially a conductor inserted into a plasma. The conductor is then biased with a voltage and the current to the probe measured as a function of bias voltage [Hutchinson, 1987]. However, analysis of Langmuir probe measurements is non-trivial. Depending on the experimental conditions, there are many factors that must be considered. Typically, stationary Maxwellian distributions of plasma species are assumed because analysis of Langmuir probe measurements in drifting, non-Maxwellian, or collisional, plasmas requires comparison with complex theoretical models. Excellent reviews of the theory and operation of Langmuir probes can be found in the literature Chen [1965], Hershkowitz [1989], Light [2000], and Hutchinson [1987]. All of the basic features of the Langmuir probes used in these experiments have been discussed previously [Balkey, 2000; Keiter et al., 2000] and only the most significant aspects of the probe theory, design, and data analysis will be presented here.

\subsubsection{Langmuir Probe Theory}

For a Langmuir probe, the relationship between the biasing voltage and the collected current is referred to as an I-V characteristic or an I-V trace. An ideal I-V trace is shown in Figure 37.

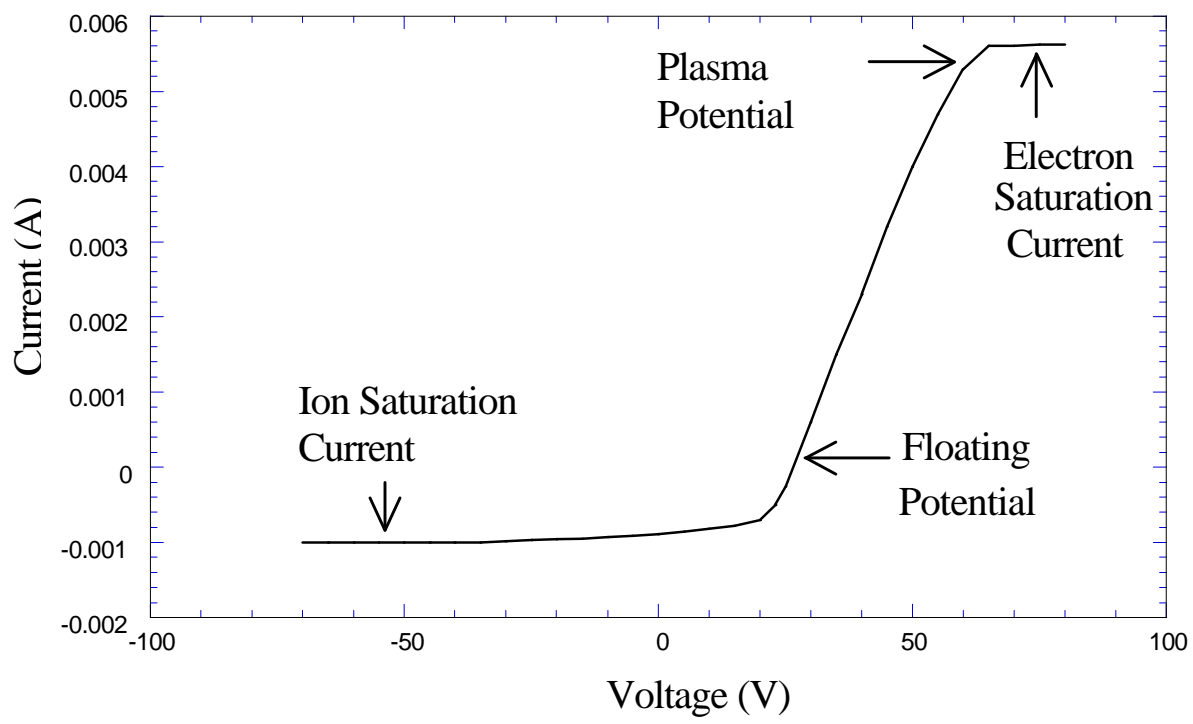

Figure 37: Hypothetical Langmuir probe trace [Keiter et al., 2000]. 
When a Langmuir probe is placed in a plasma, the probe will charge to the floating potential. The floating potential is the voltage at which no current flows to the probe, i.e. the electron and ion fluxes are the same. For a typical quasi-neutral plasma, the floating potential is negative because the electrons are more mobile than the ions. Once the probe potential becomes negative, it impedes the electron flux until the electron and ion fluxes are equal. Biasing the probe more negatively than the floating potential causes the ion flux to increase by attracting the ions and repelling the electrons. As the negative bias increases, the ion current will reach a maximum possible current in an ideal case. This maximum ion current is called the ion saturation current and it depends on the size of the probe, the plasma density and the electron temperature. A similar process occurs when the Langmuir probe is biased more positive than the floating potential. Large positive biases draw larger and larger electron currents until the electron saturation current is reached. The bias potential at which slope begins to decrease near electron saturation $\left(2^{\text {nd }} \mathrm{Knee}\right)$ is called the plasma potential. As with the ion saturation current, the electron

saturation current depends on the probe size, the plasma density, and the electron temperature. The electron saturation current is much larger than the ion saturation current because of the electrons have less inertia (higher mobility) than the ions.

\subsubsection{Determination of Electron Density and Temperature from Langmuir Probe Data}

For a collisionless plasma with no magnetic field, the current in the region just above the floating potential can be approximated by [Hutchinson, 1987]:

$$
I\left(V_{o}-V_{p}\right)=n_{\infty} e A_{p}\left(\frac{k T_{e}}{m_{i}}\right)^{1 / 2}\left[\frac{1}{2}\left(\frac{2 m_{i}}{\pi m_{e}}\right)^{1 / 2} \exp \left(\frac{e\left(V_{o}-V_{p}\right)}{k T_{e}}\right)-\frac{A_{s}}{A_{p}} \exp \left(-\frac{1}{2}\right)\right],
$$

where $m_{e}$ is the electron mass, $m_{i}$ is the ion mass $A_{s}$ is the area of the sheath, $A_{p}$ is the surface area of the probe, $V_{p}$ is the plasma potential, $V_{o}$ is the applied voltage, and the second term in the bracket on the right side of Equation (3.20) is the ion saturation current. The sheath around a Langmuir probe is a region of space a few Debye lengths thick. The sheath is a region of spatially varying potential and is created when the ions in the plasma Debye shield the potential applied to the probe [Krall and Trivelpiece, 1986]. 
The two unknowns in equation (3.20) are $n_{\infty}$, the electron density far from the probe, and $T_{e}$, the electron temperature. The derivative of the current with respect to the voltage seen by the plasma constituents is:

$$
\frac{d I}{d\left(V_{o}-V_{p}\right)}=\frac{e}{k T_{e}}\left(I-I_{s i}\right)+\frac{d I_{s i}}{d\left(V_{o}-V_{p}\right)},
$$

where $I_{s i}=-e J_{i}$ and

$$
J_{i}=0.61 n_{\infty} A_{p} \sqrt{\left(k T_{e} / m_{i}\right)} .
$$

Since the ion saturation current, $I_{s i}$, is small compared to the current collected by the probe when biased above the floating potential, $d I / d\left(V_{o}-V_{p}\right)>>d I_{s i} / d\left(V_{o}-V_{p}\right)$, so $T_{e}$ can be approximated by

$$
T_{e}=e\left(I-I_{s i}\right) / \frac{d I}{d\left(V_{o}-V_{p}\right)} .
$$

Once the electron temperature is obtained from the slope of the I-V characteristic, Equation (3.22) is used to calculate the electron density of the plasma from the measured ion saturation current. Since the Langmuir probe in HELIX was not driven into electron saturation, the plasma potential, $V_{p}$, was not measured and the probe bias voltage was used to calculate the slope of the characteristic near the floating potential. Since the plasma potential is a constant value, the two methods of calculation are equivalent.

Because of the relatively high densities in the helicon sources, Langmuir probes are not often driven into electron saturation because the current at electron saturation destroys the probe. Since the measurements do not reach electron saturation, the plasma potential is not measured. However, with measurements of the electron temperature and floating potential, an estimate for the plasma potential can be calculated. For plasmas with $T_{e}>T_{i}$, there is a simple relationship between the floating potential and plasma potential. At the floating potential, the ion current, $j_{i}$, and the electron current, $j_{e}$, are given by

$$
j_{i}=0.25 n e \sqrt{8 k T_{i} / \pi m_{i}},
$$

and 


$$
j_{e}=0.25 n e \sqrt{8 k T_{e} / \pi m_{e}} \exp \left(e\left(V_{f}-V_{p}\right) / k T_{e}\right) \text {, }
$$

respectively, where $V_{f}$ is the floating potential, $V_{p}$ is the plasma potential, $k$ is Boltsmann's constant, $m_{i}$ is the ion mass, $m_{e}$ is the electorn mass, $n$ is the plasma density, $e$ is electron charge, and $T_{e}$ is the electron temperature [Sprott, 1966]. Since the Langmuir probe current is zero at the floating potential by definition, Equations (3.24) and (3.25) can be set equal and rearranged to yield

$$
V_{p}=V_{f}+\frac{k T_{e}}{2 e} \ln \left(T_{e} m_{i} / T_{i} m_{e}\right) .
$$

If $T_{i}<T_{e}, T_{i}$ can be replaced by $T_{e}$ in equation (3.24) and equation (3.25) can be arranged to determine the plasma potential [Sprott, 1966]

$$
V_{p}=V_{f}+\frac{k T_{e}}{2 e} \ln \left(m_{i} / m_{e}\right)
$$

If $T_{e}$ is constant over a finite region, the plasma potential and the floating potential differ only by a constant in that region.

\subsubsection{Magnetic Field Effects on Langmuir Probes}

Langmuir probes are often used in magnetized plasmas. Charged particle motion across magnetic field lines is restricted while motion along the field lines is unchanged. Cross field motion of the electrons is reduced compared to the ions because their gyroorbits are much smaller than those of the ions (assuming the electrons and ions are approximately the same temperature). Thus, the electron saturation current decreases with a stronger magnetic field [Hutchinson, 1987]. For cylindrical probes, the importance of magnetic field effects scales with the ratio of the gyro-radius to the probe radius. When this ratio is much less than one for a particle species, then that species is impeded from reaching the probe and the equations (3.20) through (3.23) must be modified by including collisions and estimates of cross field transport [Hutchinson, 1987]. For a $1000 \mathrm{G}$ magnetic field in HELIX and ion temperature of $0.3 \mathrm{eV}$, the ion gyro-radius is roughly $3.5 \mathrm{~mm}$. Since the ion gyro-radius is still larger than the probe tip size, the ion dynamics are not significantly affected by the magnetic field and only the 
ion saturation current is reduced by about $20 \%$ as a result of the reduction in ion gyroradius [Hutchinson, 1987].

\subsubsection{Langmuir Probe Apparatus}

Intense RF fields in a plasma can influence the I-V trace of a Langmuir probe by distorting the electron retardation region, especially near the floating potential [Krall and Trivelpiece, 1986]. Near the floating potential, the RF electric fields accelerate and decelerate the electrons to the probe. At high frequencies, a time-averaged signal due to the RF fields can change the floating potential. If the fields are strong enough, accelerated electrons can cancel the ion current in the saturation region making the signal look like a Maxwellian distribution with a hot tail (Figure 38) [Boschi and Magistrelli, 1962; Krall and Trivelpiece, 1986; Light et al., 2001]. Sudit and Chen developed a method of probe construction to reduce RF effects on Langmuir probe measurements [Sudit and Chen, 1994]. Their method is similar to a method developed by Godyak et al. [1992] for use in inductively coupled plasma processing systems.

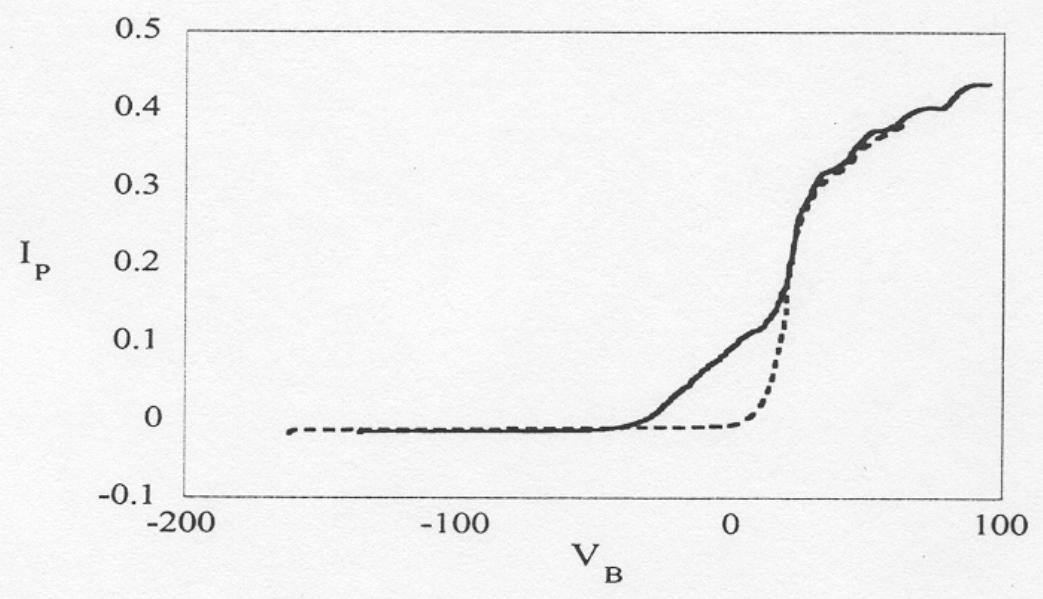

Figure 38: Langmuir probe traces with compensation (dashed line) and without compensation (solid line) [Light et al., 2001].

In the Sudit and Chen design, two modifications are made to a standard Langmuir probe. The first is the addition of a floating electrode [Sudit and Chen, 1994]. The 
electrode is exposed to the plasma potential fluctuations and connected to the Langmuir probe tip via a large capacitor. This helps to lower the sheath impedance and forces the Langmuir probe tip follow the plasma potential oscillations, thereby reducing the distortion in the trace. The second modification is a chain of RF chokes. The chokes are placed after the probe tip, but before the current is measured. The chokes increase the impedance of the circuit at the RF frequency. The Langmuir probe used in HELIX and LEIA includes a floating electrode placed near the Langmuir probe tip, but the secondary tip is not directly exposed to the plasma. A series of five RF chokes are placed after the Langmuir probe tip inside the head of the probe [Keiter, 1999; Balkey, 2000]. The impedance as a function of frequency for a typical Langmuir probe used in LEIA is shown in Figure 39.

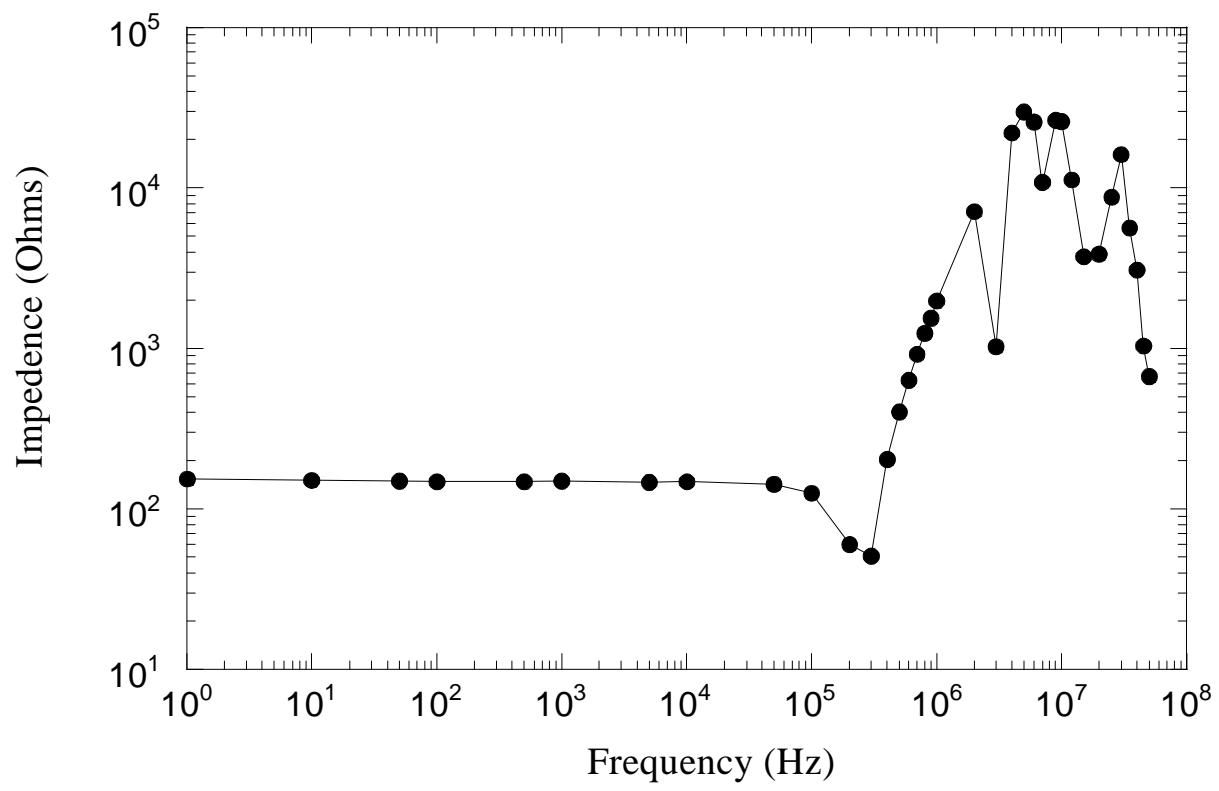

Figure 39: Typical frequency response of the Langmuir probe used in LEIA [Keiter, 1999].

The Langmuir I-V traces are measured with a Keithley 2400 SourceMeter. The source meter both applies a high output impedance bias to the Langmuir probe and measures the collected current with a high input impendence amplifier. A $75 \mathrm{~V}$ battery is placed inline with the Langmuir probe so that the source meter can sweep from 25 to 100 $\mathrm{V}$ to produce a -50 to $25 \mathrm{~V}$ sweep. The addition of the battery allows the SourceMeter to supply a single voltage polarity throughout the sweep. Previously it was observed that 
when the SourceMeter switched polarities at a bias voltage of $0 \mathrm{~V}$, the ambient RF noise in the laboratory caused a large pulse of current to appear at the output of the current amplifier. The Source Meter was controlled with a LabWindows ${ }^{\mathrm{TM}}$ program that transmitted commands and acquired data through the GPIB interface. A schematic drawing of the Langmuir probe used in HELIX is shown in Figure 40. A typical Langmuir probe trace from LEIA is shown in Figure 42.

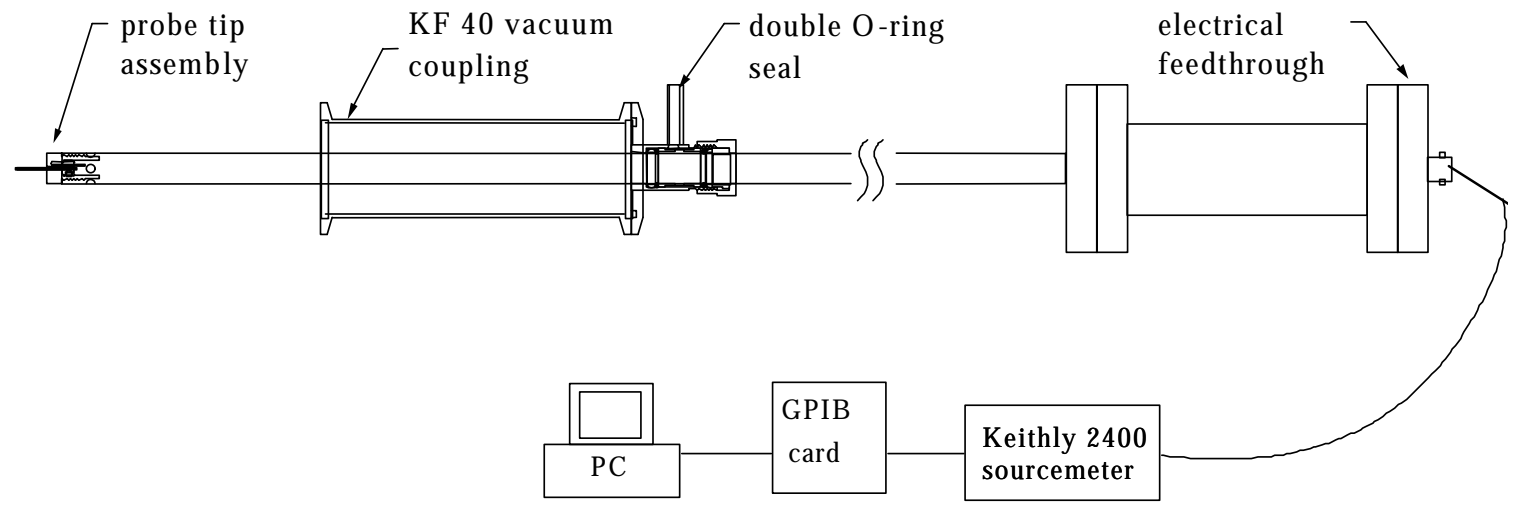

Figure 40: Schematic drawing of the Langmuir probe and measurement circuit [Keiter, 1999].

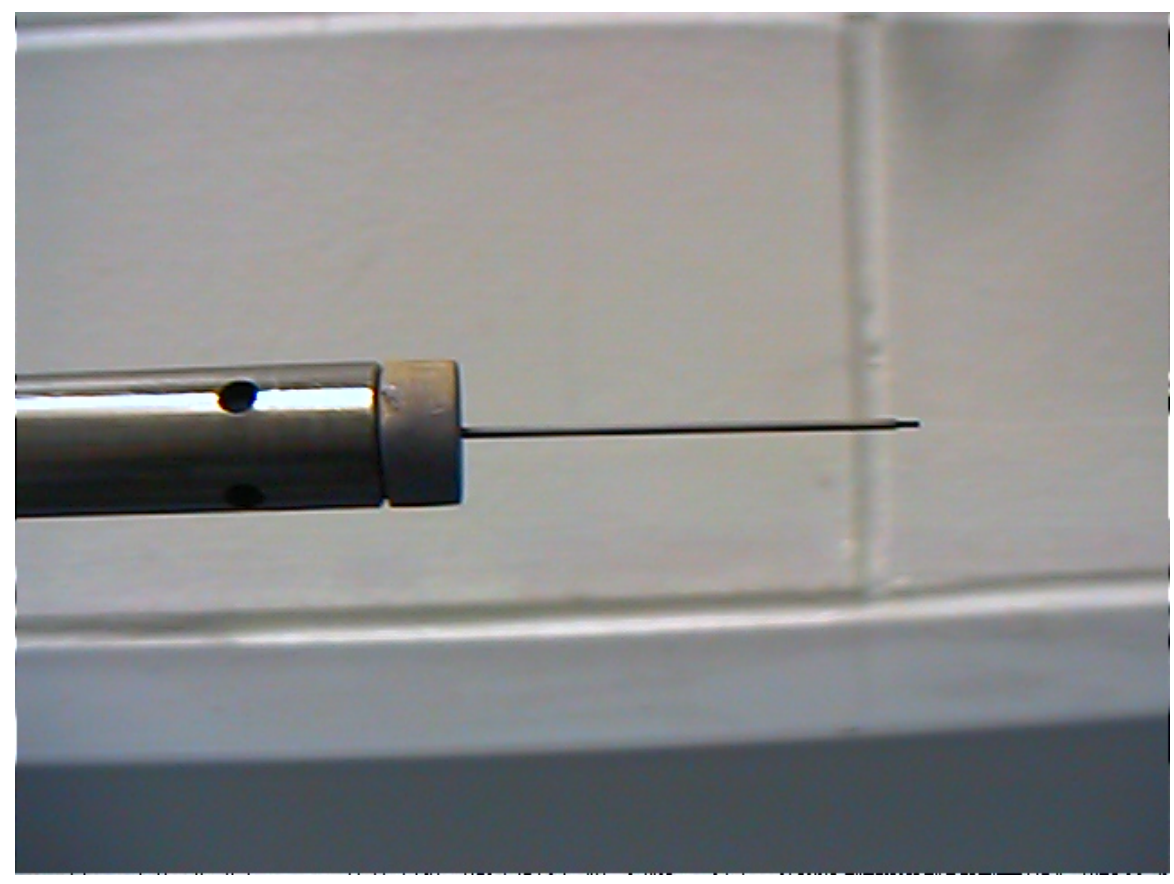

Figure 41: Photograph of the Langmuir probe head. The exposed graphite tip is $2 \mathrm{~mm}$ long and runs the length of the alumina tube into the boron-nitride cap [Keiter, 1999]. 


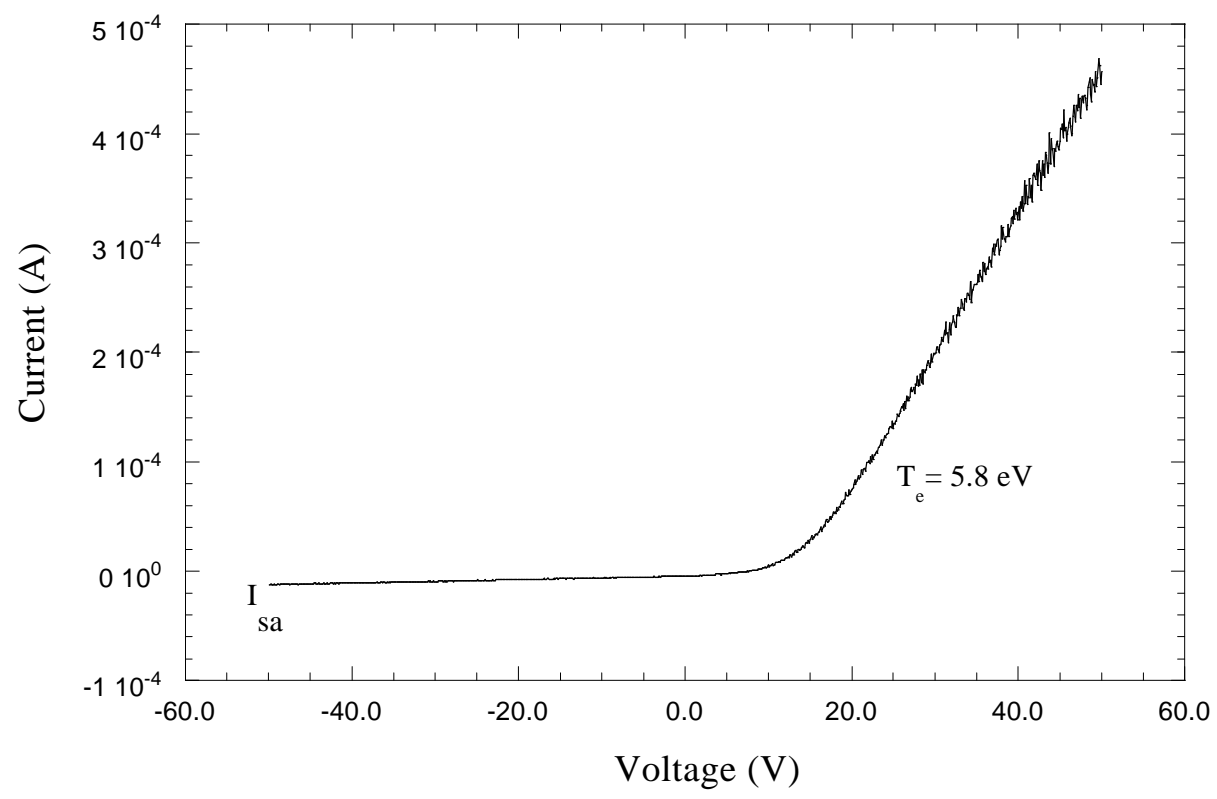

Figure 42: Typical Langmuir probe trace in LEIA [Keiter, 1999].

Electron temperatures in HELIX are typically $4-6 \mathrm{eV}$. These values are somewhat higher than the 3-5 eV temperatures reported by other helicon groups. Possible causes of the discrepancy include a net drift of the electrons into LEIA and difficulty with attaining complete RF compensation over the wide range of RF frequencies used in HELIX experiments.

\subsection{Electrostatic Fluctuation Measurements}

The electrostatic probe is essentially a two tip Langmuir probe. The two tips are separated spatially to measure differences in floating potential. The electrostatic probe has no RF compensation because the objective was to measure fluctuations in the floating potential at frequencies up to the RF driving frequency.

\subsubsection{Electrostatic Probe Theory}

Since the conducting tips of the electrostatic probe are unbiased, they charge up to the voltage at which no current flows to the probe, i.e. the floating potential. When additional charged particles are driven to the probe by changes in the nearby plasma potential arising from electrostatic waves, the particle fluxes to the probe change. The changes in particle flux cause changes in the floating potential. By measuring the 
floating potential fluctuations at two different spatial locations, the local electric field strength can be determined from $\boldsymbol{E}=\Delta \varphi / d$ where $\Delta \varphi$ is the difference in floating potential, $\varphi_{1}\left(x_{1}\right)-\varphi_{2}\left(x_{2}\right)$, and $d$ is the tip separation distance, $x_{1}-x_{2}$. The electrostatic probe can also be used to measure the wavelength of traveling electrostatic waves by determining the phase difference between the two tips and using the separation distance.

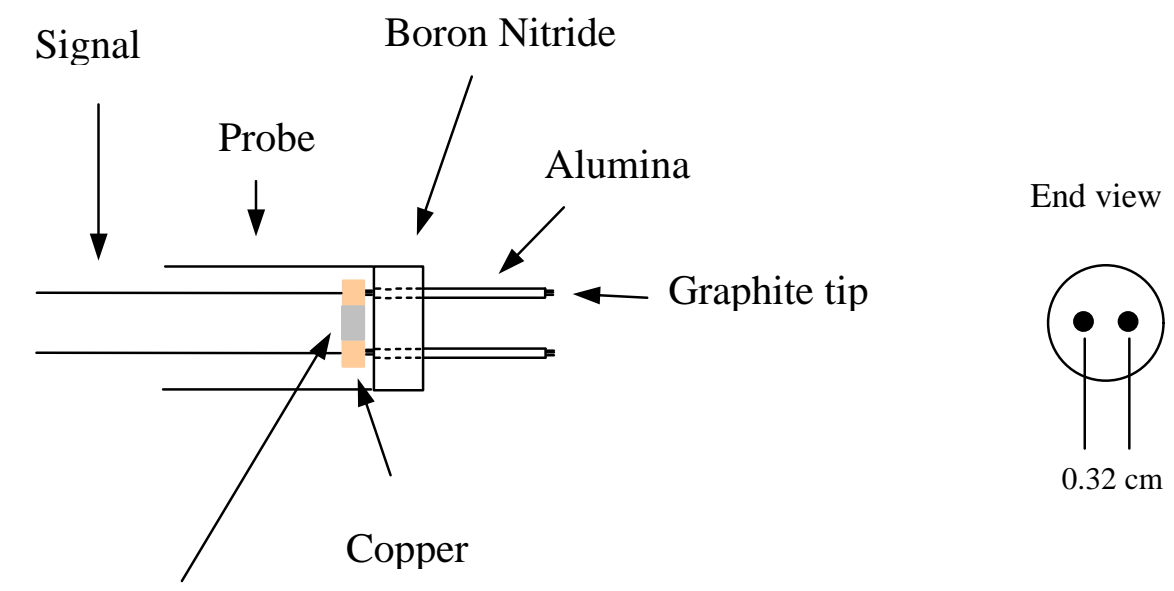

Boron Nitride

Figure 43: Schematic diagram of electrostatic probe.

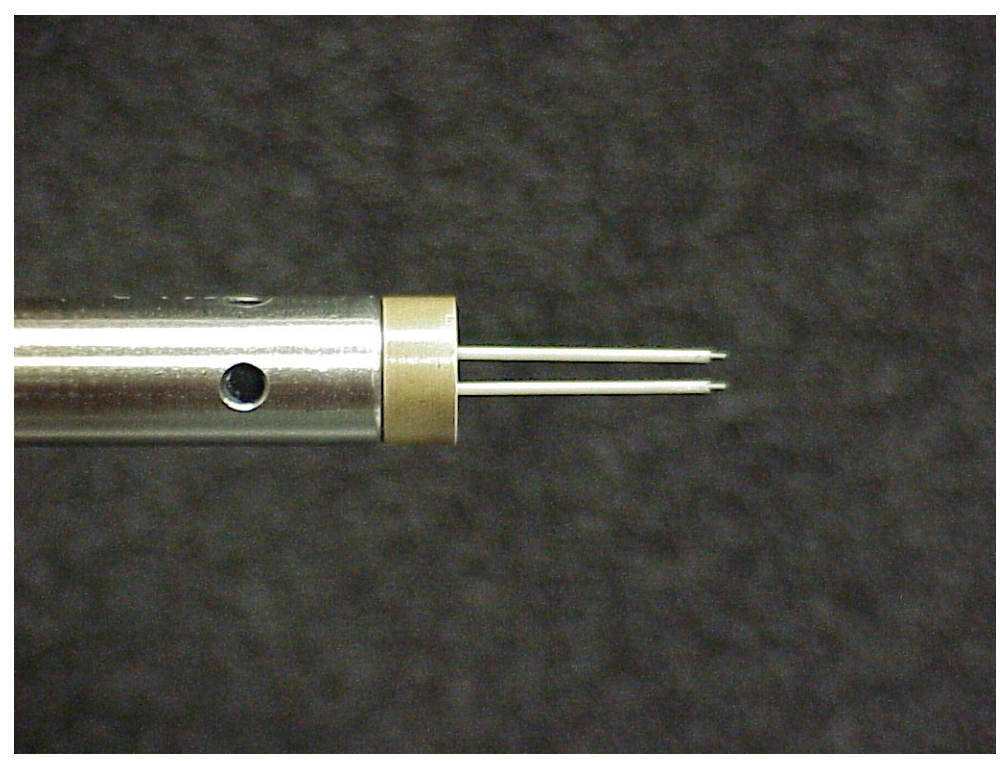

Figure 44: Picture of electrostatic probe. 


\subsubsection{Electrostatic Probe Apparatus}

The Electrostatic probe consists of two graphite tips $2 \mathrm{~mm}$ long and $0.5 \mathrm{~mm}$ in diameter. The tips are $0.32 \mathrm{~cm}$ apart. Each graphite tip protrudes from an alumina shaft $1.5 \mathrm{~cm}$ long that is mounted in a Boron Nitride cap. Inside the cap, the graphite and the signal wire were soldered to a piece of copper and the wire connected to a vacuum BNC feed through. The signal from each tip was connected to a single high impedance channel, $10 \mathrm{M} \Omega$, on the Tektronics TDS430 digital oscilloscope. Signals from each tip were recorded by the digital oscilloscope and transferred via a GPIB bus to a PC.

\subsubsection{Electrostatic Probe Data Analysis}

The power spectrum of electric field fluctuations can be obtained by calculating the FFT of the difference between the two signals divided by the tip separation distance. The wave number and frequency spectra of electrostatic waves are obtained by calculating the spectral density function using the signals from the two tips as described in Section 3.2.3. An important issue with these electrostatic probe measurements is the possibility of one tip shadowing the other. To reduce the effects of tip shadowing and to increase the effective spatial resolution of the probe, the electrostatic probe measurements of wave number were performed at multiple angles with respect to the magnetic field. 


\section{Chapter 3 References}

Assadi, S., Measurement of Magnetic Turbulence Structure and Nonlinear Mode Coupling of Tearing Fluctuations in the Madison Symmetric Torus Reversed Field Pinch, Ph. D. thesis, University of Wisconsin - Madison, Madison (1994).

Balkey, M.M., Optimization of a Helicon Plasma Source for Maximum Density with Minimal Ion Heating, Ph. D. thesis, West Virginia University, Morgantown (2000).

Bashkin, S., and Stoner, J.O., Atomic Energy Levels and Grotian Diagrams: Volume II. Sulfur I - Titanium XXII, North Holland Publishing Company, Amsterdam, 1978.

Beall, J.M., Kim, Y.C., and Powers, E.J., Estimation of wavenumber and frequency spectra using fixed probe pairs, J. Appl. Phys., 53, 3933 (1982).

Bendat, J.S., and Piersol, A.G., Engineering Applications of Correlation and Spectral Analysis, John Wiley \& Sons, New York, 1980.

Boivin, R.F., Line Broading Mechanisms, PL-039, West Virginia University, Morgantown (1999).

Boivin, R.F., Zeeman Splitting for LIF Transtions and De-convolution Technique to Extract Ion Temperatures., PL-050, West Virginia University, Morgantown (2002).

Boivin, R.F., Kline, J.L., Scime, E.E., and Cohen, S.A., Simple and Inexpensive Diode Base LIF diagnostic for Ar Plasmas, Rev. Sci. Instrum., to be submitted (2002).

Boschi, A., and Magistrelli, F., Nuovo Cimento, 29, 487 (1962).

Chen, F.F., Electric Probes, in Plasma Diagnostic Techniques, edited by R.H. Huddlestone, and S.L. Leonard, pp. 113 Academic Press, New York, 1965.

Ezekiel, S., and Wiess, R., Laser Induced Fluorescence in a Molecular Beam of Iodine, Phys. Rev. Lett., 20, 91 (1968).

Gerstenkorn, S., and Luc, P., Absolute Iodine (I2) Standards Measured by Means of Fourier Transform Spectroscopy, Revue de Physique Appliquee, 14, 791 (1979).

Gerstenkorn, S., and Luc, P., Atlas du Spectre D'Absorption de la Molecule de L'Iodine Entre 14800 - $20000 \mathrm{~cm}-1$, ( Editions du C.N.R.S., 15, quai Anatole-France, 75700 Paris) (1980).

Godyak, V.A., Piejak, R.B., and Alexandrovich, B.M., Measurement of electron energy distribution in low-pressure RF discharges, Plasma Sources Sci. Technol., 1, 36 (1992).

Hershkowitz, N., How Langmuir Probes Work, in Plasma Diagnostics, Discharge Parameters, and Chemistry, edited by O. Auciello, and D.L. Flamm, Acedemic Press, 1989.

Hill, D.H., Fornaca, S., and Wickham, M.G., Single frequency scanning laser as a plasma diagnostic, Rev. Sci. Instrum., 54, 309 (1983).

Hutchinson, I.H., Principles of Plasma Diagnostics, Cambridge University Press, 1987.

Keiter, P.A., Experimental Investigation of Ion Temperature Anisotropy Driven Instabilities in a High Beta Plasma, Ph. D. thesis, West Virginia University, Morgantown (1999). 
Keiter, P.A., Scime, E.E., Balkey, M.M., Boivin, R., Kline, J.L., and Gary, S.P., Betadependent upper bound on ion temperature anisotropy in a laboratory plasma, Phys. Plasmas, 7, 779 (2000).

Kline, J.L., Scime, E.E., Keiter, P.A., Balkey, M.M., and Boivin, R.F., Ion heating in the HELIX helicon plasma source, Phys. Plasmas, 6, 4767 (1999).

Krall, N.A., and Trivelpiece, A.W., Principles of plasma physics, San Francisco Press, 1986.

Light, M., Chen, F.F., and Colestock, P.L., Low frequency electrostatic instability in a helicon plasma, Phys. Plasmas, 8, 4675 (2001).

Light, M., Sudit, I.D., Chen, F.F., and Arnush, D., Axial propagation of helicon waves, Phys. Plasmas, 2, 4094 (1995).

Light, M.E., A study of Low Frequency Instability and Transport in a Helicon Plasma, $\mathrm{Ph}$. D. thesis, University of California - Los Angeles, Los Angeles (2000).

Lochte-Holtgreven, W., Plasma Diagnostics, American Institute of Physics, New York, 1995.

Loewenhardt, P.K., Blackwell, B.D., and Beichao, Z., A simple miniature magnetic probe with inherent electrostatic rejection (for plasma diagnostics), Rev. Sci. Instrum., 64, 3334 (1993).

McChesney, J.M., Observations of Stochastic Ion Heating by Low Frequency Drift Waves, Ph. D. thesis, California Institute of Technology (1989).

Sarfaty, M., De Souza Machado, S., and Skiff, F., Direct determination of ion wave fields in a hot magnetized and weakly collisional plasma, Phys. Plasmas, 3, 4316 (1996).

Scime, E.E., Keiter, P.A., Zintl, M.W., Balkey, M.M., Kline, J.L., and Koepke, M.E., Control of ion temperature anisotropy in a helicon plasma, Plasma Sources Sci. Technol., 7, 186 (1998).

Severn, G.D., Edrich, D.A., and McWilliams, R., Argon ion laser-induced fluorescence with diode lasers, Rev. Sci. Instrum., 69, 10 (1998).

Skiff, F., and Anderegg, F., Direct observation of plasma dielectric motion, Phys. Rev. Lett., 59, 896 (1987).

Skiff, F., Bachet, G., and Doveil, F., Ion dynamics in nonlinear electrostatic structures, Phys. Plasmas, 8, 3139 (2001).

Sprott, J.C., Electrostatic Probe Techniques, PLP 88, University of Wisconsin, Madison (1966).

Stern, R.A., and Johnson, J.A., III, Plasma ion diagnostics using resonant fluorescence, Phys. Rev. Lett., 34, 1548 (1975).

Sudit, I.D., and Chen, F.F., RF compensated probes for high-density discharges, Plasma Sources Sci. Techol., 3, 162 (1994).

Yardley, Y.T., and Moore, C.B., Journal of Chemical Physics, 45, 1066 (1966). 


\section{Chapter 4: Helicon Source Theory}

Perhaps the greatest impediment to the development of a comprehensive model capable of explaining the operational characteristics of a typical helicon source is lack of any agreement amongst helicon source researchers as to what constitutes a "typical" helicon source. The capacitively coupled plasma source community has successfully developed a common plasma source through the auspices of the Gaseous Electronics Conference, the GEC cell (The detailed designs plans for a GEC cell are publicly available [Greenberg et al., 1990]). Researchers at one institution can field diagnostics on their own GEC cell and then combine their measurements with researchers elsewhere to obtain a comprehensive understanding of GEC cell physics. Because helicon sources are constructed for a wide variety of purposes, e.g., plasma processing studies [Perry et al., 1991; Chen, 1992; Hershkowitz et al., 1996; Okamoto et al., 1999; Chen et al., 2000], plasma thruster development [Chang Diaz et al., 1999; Cohen et al., 1999], basic plasma physics experiments [Scime et al., 1998; Light, 2000; Scime et al., 2000; Light et al., 2001], and space relevant plasma physics experiments [Keiter et al., 2000; Scime et al., 2000; Hanna and Watts, 2001], there are only a few common features among the wide assortment of helicon source designs. Helicon sources are cylindrical systems with a purely axial magnetic field [Boswell, 1970]. The aspect ratio (diameter/length) of helicon sources can vary from approximately unity [Lho et al., 1998] to greater than twenty [Chen and Decker, 1992]. RF power is inductively coupled into the plasma through an antenna. In most helicon sources, the antenna is mounted outside the vacuum system and the coupling is accomplished through a non-conducting section of the vacuum chamber (often Pyrex or quartz) [Boswell, 1970; Chen, 1992; Keiter et al., 1997]. For those helicon sources with internal antennas [Loewenhardt et al., 1991; Franck and Klinger, 2000; Grulke et al., 2001], insulating liners are sometimes placed between the antenna and the plasma to prevent strong capacitive coupling of the plasma to the antenna circuit [Franck and Klinger, 2000]. The range of radial boundary conditions for helicon sources with external antennas includes: tightly fitting conducting walls outside of the antenna region; insulating walls throughout the entire system [Keiter et al., 1997; Miljak and Chen, 1998; Guo et al., 1999]; and conducting walls with a vacuum gap between the 
plasma and the walls [Shoji et al., 1993; Shinohara et al., 1995; Hanna and Watts, 2001; Kline et al., 2002]. The axial boundaries play an important role in helicon source operation and various designs have been studied: conducting boundaries at each end [Gilland et al., 1998]; conducting boundary at one end with the other end opening into a much larger vacuum chamber [Degeling et al., 1996; Sakawa et al., 1997; Keiter et al., 2000]; and no boundaries at either end [Nakamura et al., 1995; Takeno et al., 1995; Scime et al., 2001].

The RF frequencies used for helicon sources typically range between $7 \mathrm{MHz}$ and 28 MHz [Boswell, 1984; Chen, 1992; Chen and Decker, 1992; Keiter et al., 1997; Kwak et al., 1997; Balkey et al., 2001], but some groups have operated helicon sources at frequencies as high $100 \mathrm{MHz}$. Although most helicon sources operate at a fixed RF frequency to simplify impedance matching issues, a number of groups have explored helicon source properties as a function of RF frequency [Keiter et al., 1997; Kwak et al., 1997; Yun et al., 1997]. Historically, helicon sources operated at magnetic field strengths of 400 to 4000 Gauss [Boswell, 1984; Keiter et al., 1997; Cohen et al., 1999], but recent experiments at weak magnetic fields of 50 to 100 Gauss are considered to be "helicon source" experiments by the helicon source research community [Boswell, 1984; Boswell and Vender, 1995; Chen et al., 1997; Degeling et al., 1998]. In addition to the wide range of aspect ratios, RF frequencies, and magnetic field strengths, helicon source experiments have used different gasses: argon [Boswell, 1984; Scime et al., 1998]; helium [Boivin et al., 2001]; oxygen [Mieno et al., 1996]; xenon [Yun et al., 1997]; silicon [Durandet et al., 1997]; and hydrogen [Chang Diaz et al., 1999], a wide range of neutral pressures: 0.3 mTorr [Boswell and Porteous, 1987] to 100 mTorr [Boivin et al., 2001]; and a wide range of RF powers: 100 Watts [Shinohara and Soejima, 1998] to $<100 \mathrm{~kW}$ [Shats et al., 1997]. Therefore, it is surprising that any consensus has been reached concerned the basic physics of helicon sources.

Two different approaches have been used to formulate models of the electromagnetic wave fields in helicon sources and to try and understand the efficient coupling of the RF power into the plasma. The first approach, developed by Blevin [1966], is a single fluid calculation. Massive (fixed) ions and no DC currents in the plasma are assumed. The only currents in the plasma are due to the electron Hall current, $\mathbf{J}_{1}=-n e\left(\mathbf{E}_{1} \times \mathbf{B}_{\mathbf{o}} / B_{\mathrm{o}}{ }^{2}\right)$ 
where the 1 subscript represents wave or perturbed quantities and the terms with the o subscript are the static quantities. Physically, the electrons oscillate at the $\mathbf{E}_{1} \times \mathbf{B}_{\mathbf{0}} \mathrm{drift}$ velocity where $\mathbf{E}_{1}$ is the wave electric field and $\mathbf{B}_{\mathbf{0}}$ is the applied steady state magnetic field. The oscillating motion of the electron guiding centers in the oscillating wave field generates an oscillating, perpendicular (to $\mathbf{B}_{\mathbf{0}}$ ), current at the wave frequency. From the Hall current and Maxwell's equations in cylindrical coordinates, a second order differential equation for the amplitude of the $z$ component of the wave magnetic field, $\mathbf{B}_{1 z}$, is derived assuming the perturbations are of the form $\mathbf{B}_{1}(\mathrm{r}) \exp \left[i\left(m \theta+k_{\|} z-\omega t\right)\right]$ where $m$ is the azimuthal mode number, $\omega$ is the wave frequency, and $k_{\|}$is the wave number parallel to the applied magnetic field [Blevin and Christiansen, 1966; Chen et al., 1994]:

$$
B_{1 z}^{\prime \prime}+f(r) B_{1 z}^{\prime}+g(r) B_{1 z}=0
$$

where $\quad=\partial / \partial r$,

$$
\begin{gathered}
f(r)=\frac{1}{r}-\frac{2 \alpha \alpha^{\prime}}{k_{\perp}^{2}}, \\
g(r)=\frac{k_{\perp}^{2}}{\gamma}-\frac{m^{2}}{r^{2}}-\frac{m \alpha^{\prime}}{k_{\|} \gamma r}\left(1+\frac{2 k_{\|}^{2} \gamma^{2}}{k_{\perp}^{2}}\right), \\
\alpha(r)=\frac{\omega \mu_{o} e}{B_{o} k_{\|}} n(r), \\
\gamma=1-\left(\frac{\omega}{c k_{\|}}\right)^{2}, \\
k_{\perp}^{2}=\alpha^{2}-k_{\|}^{2},
\end{gathered}
$$

$k_{\perp}$ is the wave number perpendicular to the magnetic field, $n(r)$ is the density as a function of radius (r), $e$ is the unit charge of an electron, $B_{o}$ is the applied magnetic field, $c$ is the speed of light, and $\mu_{o}$ is the permeability constant.

Application of either conductive $\left(\hat{n} \cdot \mathbf{B}_{1 z}(r=a)=0\right)$ or insulating radial boundary $\left(\hat{n} \cdot \mathbf{J}_{1 z}(r=a)=0\right)$ conditions at the vacuum chamber walls yield Bessel function solutions to Equation (4.1) for uniform density profiles. For small aspect ratio helicon 
sources, the perpendicular wavelength of the wave is often chosen to match the lowest order radial mode of the system [Blevin and Christiansen, 1966; Chen et al., 1994]. For the $m=+1$ helicon wave in a small aspect ratio, uniform plasma, the dispersion relation reduces to [Keiter, 1999]

$$
\omega=\frac{\omega_{c e} k_{\|} k c^{2}}{\omega_{p e}^{2}} .
$$

Finite aspect ratio solutions [Keiter et al., 1997] yield more complex wave field structure, but generally similar dispersion relations. The amplitudes of $\mathbf{B}_{1 r}$ and $\mathbf{B}_{1 \theta}$ are then obtained from $\mathbf{B}_{1 z}$ (Equations (4.8) and (4.9)) and constraints on the divergence and curl of the wave magnetic field. For arbitrary density profiles, solutions for the wave amplitudes are relatively easy to obtain using [Chen et al., 1994]

$$
k_{\perp}^{2} B_{1 r}=\frac{i m \alpha(r)}{r} B_{1 z}+i k_{\|} \gamma B_{1 z}^{\prime}
$$

and

$$
k_{\perp}^{2} B_{1 \theta}=-\alpha(r) B_{1 z}^{\prime}-\frac{m k_{\|} \gamma}{r} B_{1 z}
$$

where all definitions are the same as for $\mathbf{B}_{1 \mathrm{z}}$.

Because the single fluid approach ignores ion motion, it is only applicable for RF frequencies well above the lower hybrid frequency [Davies, 1970]. The electron inertia is typically neglected, making the results valid for densities greater than $10^{13} \mathrm{~cm}^{-3}$ even if the wave frequency is much less than the electron cyclotron frequency [Davies, 1970]. Electron inertia also plays an important role on the propagation of higher radial modes [Boswell, 1972].

Given that fast waves in the whistler wave regime are natural modes of oscillation in plasmas with parameters typical of helicon sources, it is not surprising that bounded whistler waves (helicon waves) are observed in helicon sources. The mere presence of helicon waves, however, does not prove that helicon waves play any role in coupling RF power into the plasma (efficient density production) or ion heating. Not only do corrections for ion motion, collisions, and electron inertia change some of the details of 
the helicon wave magnetic field components, they also introduce additional natural modes of oscillation (new waves) in the plasma.

Solution of the hot plasma dielectric tensor in a homogeneous slab plasma for typical helicon plasma parameters yields three distinct natural modes of oscillation in the plasma: the fast (or whistler) wave, the slow (or Trivelpiece-Gould) wave, and the ion Bernstein (or warm) wave [Stix, 1965]. With the addition of radial boundary conditions, the fast wave becomes the helicon wave described by Equation (4.7). Axial boundary conditions permit only certain parallel wavelengths and further limit the possible fast wave modes that can be launched into the plasma [Shamrai, 1998; Chi et al., 1999]. Although the slow wave only becomes the Trivelpiece-Gould (TG) wave [Trivelpiece and Gould, 1959] in a bounded plasma, the slow wave is often referred to as the TG wave in the helicon source literature [Shamrai and Taranov, 1995; Cho, 1996; Chen and Arnush, 1997; Chen et al., 1997; Cho and Kwak, 1997; Borg and Boswell, 1998; Mouzouris and Scharer, 1998; Arnush, 2000; Shinohara and Yonekura, 2000; Schlüter et al., 2001; Shivarova and Tarnev, 2001; Kline et al., 2002]. Because finite ion temperature effects are almost universally ignored in models of helicon source plasmas, the role of the ion Bernstein wave in helicon sources has received no serious theoretical attention.

The second approach used for modeling helicon source plasmas combines solutions to the cold plasma dielectric tensor that describe wave propagation in the plasma with solutions to Maxwell's equations for the vacuum fields and antenna currents [Shamrai and Taranov, 1995; Arnush, 2000; Cho, 2000]. At the interfaces (plasma-vacuum, vacuum-wall), the wave solutions are forced to satisfy the boundary conditions. The boundary conditions result in what are essentially eigenmodes for wave propagation. Because the full cold plasma dielectric tensor is used, electron inertia and ion motion effects can be explicitly included in the calculation and finite temperature effects are ignored. It is important to note that in almost every application of this approach, the physical model assumes that the antenna extends along the entire length of the plasma and is surrounded by a conducting boundary [Cho, 1996; Shamrai and Taranov, 1996], two conditions not realized in most helicon experiments.

Although ion motion effects are included in the cold plasma dielectric tensor, Arnush [2000] and Shamrai and Taranov [1995] ignore ion motion effects in their calculations by 
assuming the RF driving frequency is much greater than the lower hybrid frequency. This assumption ignores the resonant behavior of the slow wave at the lower hybrid frequency and ignores the fact that many helicon sources operate at RF frequencies very close to the lower hybrid frequency [Boswell, 1984; Kwak et al., 2000; Balkey et al., 2001]. Arnush [2000] and Shamrai and Taranov [1995] concluded from their calculations that the slow wave should be excited in helicon plasmas and would be strongly damped at the plasma edge; thereby providing a mechanism for efficient RF power absorption in helicon plasmas.

The only published theoretical treatment of helicon sources that includes ion motion effects on fast and slow wave propagation is the work of Cho [2000]. Cho calculates the expected wave numbers, wave field structure, and power absorption in a helicon source near the lower hybrid resonance. However, singularities in the equations force him to use rough approximations in his zeroth order calculations. Another failing of Cho's, and the other cold plasma dielectric treatments, is the use of uniform plasma densities to avoid the difficulties associated with a radially varying dielectric tensor. Since no existing model of wave excitation and damping in helicon sources includes all of the effects that experiments suggest are important, the predictions of the different models have to be carefully considered in light of the assumptions used in the calculations. Although Cho's calculations include ion motion effects, the ions are assumed to exist at room temperature $(0.02 \mathrm{eV})$ and only introduce small corrections to the dielectric tensor. There are no theoretical calculations that have examined the possible effects of $1.0 \mathrm{eV}$ ions on wave propagation and RF power absorption in helicon plasmas.

\subsection{Cold Plasma Theory for Helicon Sources}

Solving the cold plasma dispersion relationship including ion terms and collisions, Cho found two solutions for typical helicon operating parameters [Cho, 2000]. The first solution is that of the fast wave or the helicon wave, a bounded, right hand circularly polarized electromagnetic, whistler wave. Experimental measurements confirm that electromagnetic waves with wave fields consistent with helicon waves propagate downstream of the RF antenna in helicon sources [Light et al., 1995]. The second solution is the slow wave or Trivelpiece-Gould mode. The slow wave is a surface wave 
and is heavily damped by collisions, preventing it from propagating towards the center of the plasma [Chen and Arnush, 1997]. Near the lower hybrid resonance, the slow wave becomes almost completely electrostatic. Attempts to directly measure the slow wave in a helicon plasma source have been hampered by the extremely short wavelength of the wave, $\lambda \sim 1 \mathrm{~mm}$. High frequency current profile measurements [Blackwell et al., 2001] and density profile measurements [Yun et al., 2001] have provided preliminary evidence of slow wave excitation, but no definitive experiment observations have been reported.

The cold plasma dispersion relation for typical helicon source plasma parameters is

$$
\rho^{4}-(\alpha+\beta) \rho^{2}+\alpha \beta+\gamma \delta=0,
$$

where $\rho=k_{\perp} c / \omega$ and $\alpha, \beta, \gamma$, and $\delta$ are

$$
\begin{gathered}
\alpha=\varepsilon_{1}-N^{2}-\varepsilon_{2}^{2} / \varepsilon_{1}, \\
\beta=\varepsilon_{3}\left(1-N^{2} / \varepsilon_{1}\right), \\
\gamma=N \varepsilon_{3} \varepsilon_{2} / \varepsilon_{1} \\
\delta=N \varepsilon_{2} / \varepsilon_{1}
\end{gathered}
$$

with $N=k_{\|} c / \omega . \quad \stackrel{\circ}{a}_{1}, \stackrel{\circ}{a}_{2}$, and $\stackrel{\circ}{\xi}_{3}$ are the elements of the cold plasma dielectric tensor:

$$
\begin{gathered}
\varepsilon_{1}=1+\sum_{j=e, i} \frac{\omega_{p j}^{2}\left(1+i \frac{v_{j}}{\omega}\right)}{\omega_{c j}^{2}-\omega^{2}\left(1+i \frac{v_{j}}{\omega}\right)^{2}} \\
\varepsilon_{2}=\sum_{j=e, i} \frac{\sigma_{j} \omega_{p j}^{2} \frac{\omega_{c j}}{\omega}}{\omega_{c j}^{2}-\omega^{2}\left(1+i \frac{v_{j}}{\omega}\right)^{2}}, \\
\varepsilon_{3}=1-\sum_{j=e, i} \frac{\omega_{p j}^{2} \omega^{2}\left(1+i \frac{v_{j}}{\omega}\right)}{\omega}
\end{gathered}
$$


where $j$ represents quantities of the $\mathrm{j}^{\text {th }}$ species, $\omega_{c j}$ is the cyclotron frequency, $\omega_{p j}$ is the plasma frequency, $v_{j}$ is the total collision frequency, and $\sigma_{j}$ is the sign of the charge. Near the lower hybrid resonance, $\omega_{l h}{ }^{-2}=\left(\omega_{c e} \omega_{c i}\right)^{-1}+\left(\omega_{p i}{ }^{2}+\omega_{c i}{ }^{2}\right)^{-1}$, or when $\varepsilon_{1}$ goes to zero, the perpendicular wave number goes to infinity for a collisionless plasma. The collision term, which includes ion-ion, electron-electron, ion-neutral and electron-neutral collisions, reduces the perpendicular wave number at the lower hybrid resonance to a finite value and, for large collision frequencies, slightly shifts the resonant frequency.

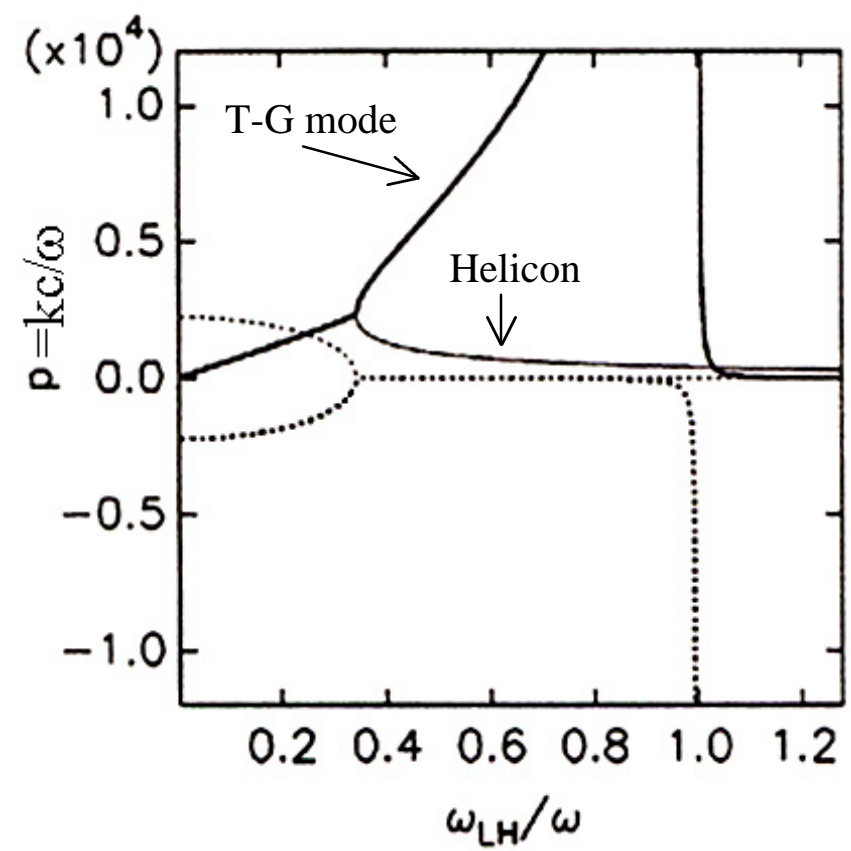

Figure 45: Normalized ( $($ ) real and (....) imaginary perpendicular wave number verses the inverse of the normalized wave frequency for a parallel wave number of $0.0785 \mathrm{rad} / \mathrm{cm}$, a frequency of $8 \mathrm{MHz}$, an argon plasma density of $4 \times 10^{12} \mathrm{~cm}^{-3}$, a magnetic field strength of 1000 Gauss, and an electron collision frequency of $\mathrm{v}_{e} / \omega=10^{-3}$ obtained from the cold plasma dispersion relation [Cho, 2000].

The normalized perpendicular wave number verses the inverse of the normalized wave frequency obtained from the cold plasma dispersion relation is shown in Figure 45. At low magnetic fields, there is only one solution to the dispersion relations. As the magnetic field strength increases, the single solution bifurcates into the fast and slow waves. For the fast, helicon wave solution, the perpendicular wave number decreases asymptotically to zero for large magnetic fields. Note that fast wave perpendicular wave 
numbers vary smoothly near the lower hybrid frequency. For the slow wave solution, the perpendicular wave number increases to infinity (in a collisionless plasma) when the wave frequency equals the lower hybrid frequency, i.e., the lower hybrid resonance. The corresponding perpendicular phase speed, $\omega / k_{\perp}$, of the slow wave goes to zero. If the wave phase speed becomes comparable to the ion thermal speed, $\mathrm{v}_{t h i} k_{\perp} / \omega \sim 1$, ion Landau damping can occur [Wong and Ono, 1981]. The potential for large perpendicular wave numbers suggests that the Landau damping of the slow wave could explain the large perpendicular ion temperatures observed in helicon sources operating near the lower hybrid frequency [Balkey et al., 2001].

\subsection{Cold Plasma Computational Model for Helicon Sources}

A simple computational model based on the cold plasma dispersion relation, including ion motion and realistic collision frequencies, was developed. The computational model computes the real and imaginary part of the perpendicular wave number for a given set of plasma parameters, e.g., density, ion temperature, electron temperature, magnetic field strength, neutral pressure, wave frequency, and parallel wave number. Boundary effects are not included in the calculations. The wave numbers were computed as a function of magnetic field strength and driving frequency for comparison with experimental results. The discussion of the computational results is divided into three sections. In Section 4.2.1, numerical solutions for the real and imaginary components of the perpendicular wave number for both the slow and fast wave in a homogeneous plasma are reviewed. In Section 4.2.2, the computational model is extended to include arbitrary density profiles by calculating solutions of the homogeneous plasma case for different values of plasma density (corresponding to different radial locations). In the last Section, 4.2.3, the maximum perpendicular wave number in the plasma is determined as a function of magnetic field and frequency for later comparison with ion temperature measurements.

\subsubsection{Solutions to Cold Plasma Dispersion Relation}

The fast and slow wave solutions for the absolute value of the real and imaginary parts of the perpendicular wave number according to Equation (4.10) as calculated with the polynomial root finder in Matlab ${ }^{\mathrm{TM}}$ are shown in Figure 46 as a function of magnetic 
field strength for typical HELIX parameters. The results are identical to Cho's calculations shown in Figure 45 [2000]. The resonance for the slow wave occurs when the RF frequency matches the lower hybrid frequency. Note that although the imaginary component of the slow wave is shown as positive in Figure 46, it is the absolute value of the imaginary component that is plotted. In fact, the slow wave is strongly damped (imaginary part $<0)$ at the lower hybrid resonance $(B \sim 850 \mathrm{G})$.

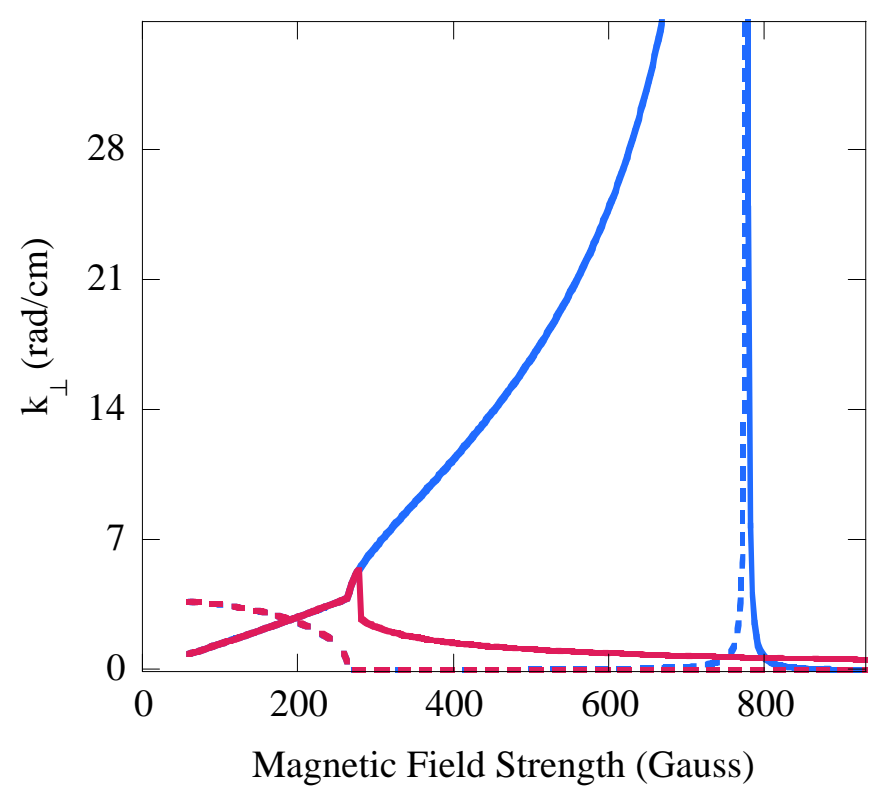

Figure 46: Solutions of the cold plasma dispersion relationship for $k_{\perp}$ with $n=5 \times 10^{12} \mathrm{~cm}^{-3}, k_{\|}=0.26 \mathrm{rad}$ $\mathrm{cm}^{-1}, f=9 \mathrm{MHz}$ and no collisions. ( $(-)$ absolute value of real $k_{\perp}$ for the slow wave. ( $\left.\cdots \cdot\right)$ ) absolute value of imaginary $k_{\perp}$ for the slow wave. ( $\rightarrow$ absolute value of real $k_{\perp}$ for the fast wave. $(\cdots .$.$) absolute value of$ imaginary $k_{\perp}$ for the fast wave.

\subsubsection{Plasma Density Effects on Solutions to the Cold Plasma Dispersion Relation}

The slow wave solutions to the cold plasma dispersion as a function of magnetic field strength are shown in Figure 47 for a collisionless plasma with a range of plasma density values, $n=1 \times 10^{11}$ to $1 \times 10^{13} \mathrm{~cm}^{-3}$. For $n=1 \times 10^{11} \mathrm{~cm}^{-3}$, the lowest density investigated, no resonant behavior appears over the range of magnetic field strengths included in Figure 47. Decreases in the plasma density decrease the lower hybrid frequency for a fixed value of magnetic field strength. In the complete expression for the lower hybrid frequency, 


$$
\frac{1}{\omega_{l h}^{2}}=\frac{1}{\omega_{c e} \omega_{c i}}+\frac{1}{\left(\omega_{p i}^{2}+\omega_{c i}^{2}\right)},
$$

the ion plasma frequency term only contributes to the lower hybrid frequency when it is comparable in magnitude to the cyclotron term. For densities greater than $5 \times 10^{11} \mathrm{~cm}^{-3}$ in HELIX, the ion plasma frequency term is only a small correction to the lower hybrid frequency. Below $5 \times 10^{11} \mathrm{~cm}^{-3}$, the lower hybrid frequency decreases with decreasing density. Therefore, the lower hybrid frequency at the plasma edge is lower than in the center of the discharge. Matching the RF frequency to the lower hybrid frequency then requires much larger magnetic field strengths (as evident in Figure 47a). The second effect is the magnetic field at which the fast and slow wave solutions diverge increases with increasing density.
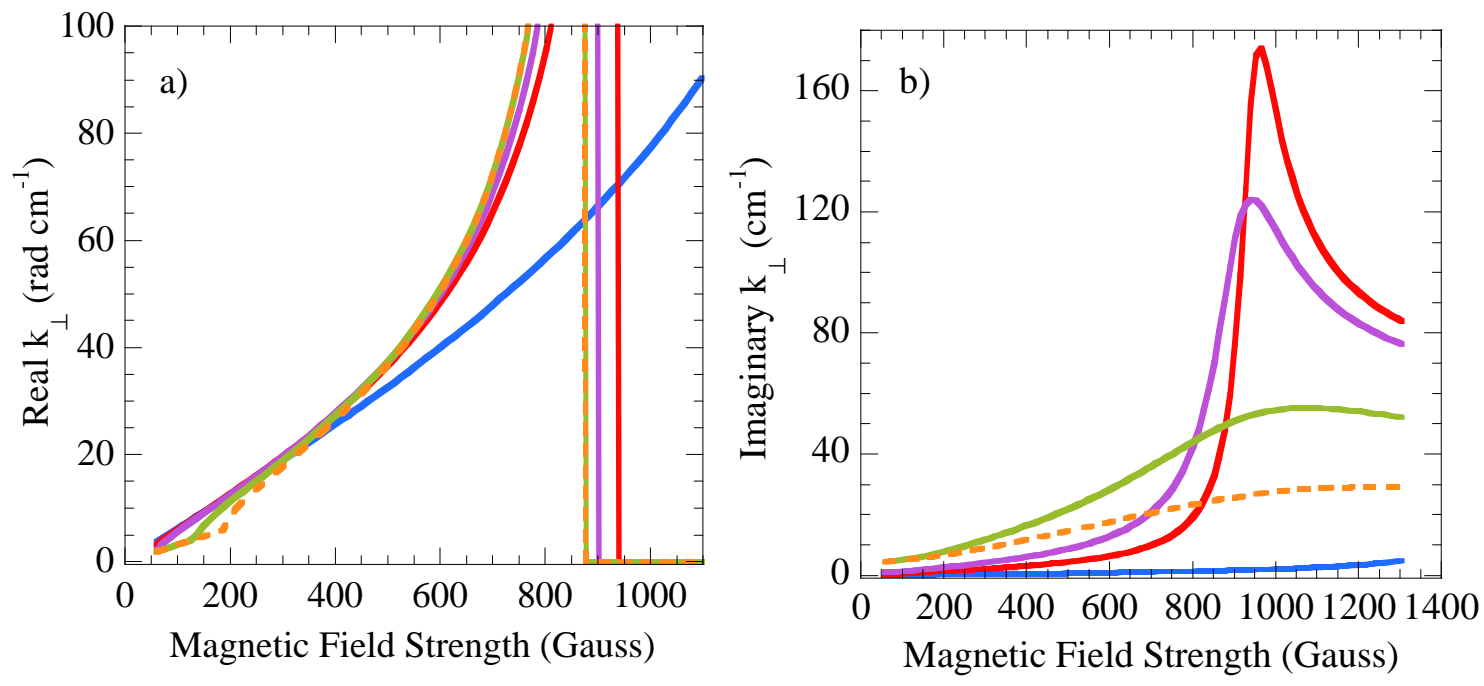

Figure 47: Absolute value of the a) real and b) imaginary components of $k_{\perp}$ for the slow wave with $k_{\|}=$ $0.26 \mathrm{rad} \mathrm{cm}^{-1}, f=9 \mathrm{MHz}$, no collisions and densities of $(-) n=1 \times 10^{11} \mathrm{~cm}^{-3},(一) n=5 \times 10^{11} \mathrm{~cm}^{-3},($ ) $n=1 \times 10^{12} \mathrm{~cm}^{-3},(-) n=5 \times 10^{12} \mathrm{~cm}^{-3}$, and $(\cdots) n=.1 \times 10^{13} \mathrm{~cm}^{-3}$ as a function of magnetic field strength.

The decrease in magnetic field strength required to match the RF frequency to the lower hybrid frequency due to increasing plasma density is also evident in calculations of the imaginary component of the perpendicular wave number shown in Figure 47b. The absolute value of the imaginary part of the wave number is greatest when the driving frequency is below the lower hybrid frequency, corresponding to heavy damping for 
waves with frequencies below the lower hybrid frequency. The magnitudes of the imaginary component are similar for all densities above $5 \times 10^{11} \mathrm{~cm}^{-3}$.

Fast wave solutions to the cold plasma dispersion relationship are shown in Figure 48 for the same range of densities used for Figure 47. Above a minimum magnetic field strength, the real part of the perpendicular wave number exhibits the expected $1 / B^{1 / 2}$ dependence of the fast wave for a fixed parallel wave number and density [Boswell, 1984; Chen, 1992]. Note that there is no perpendicular propagation of the fast wave for the lowest densities, i.e., $k_{\perp}$ goes to zero (Figure 48a).
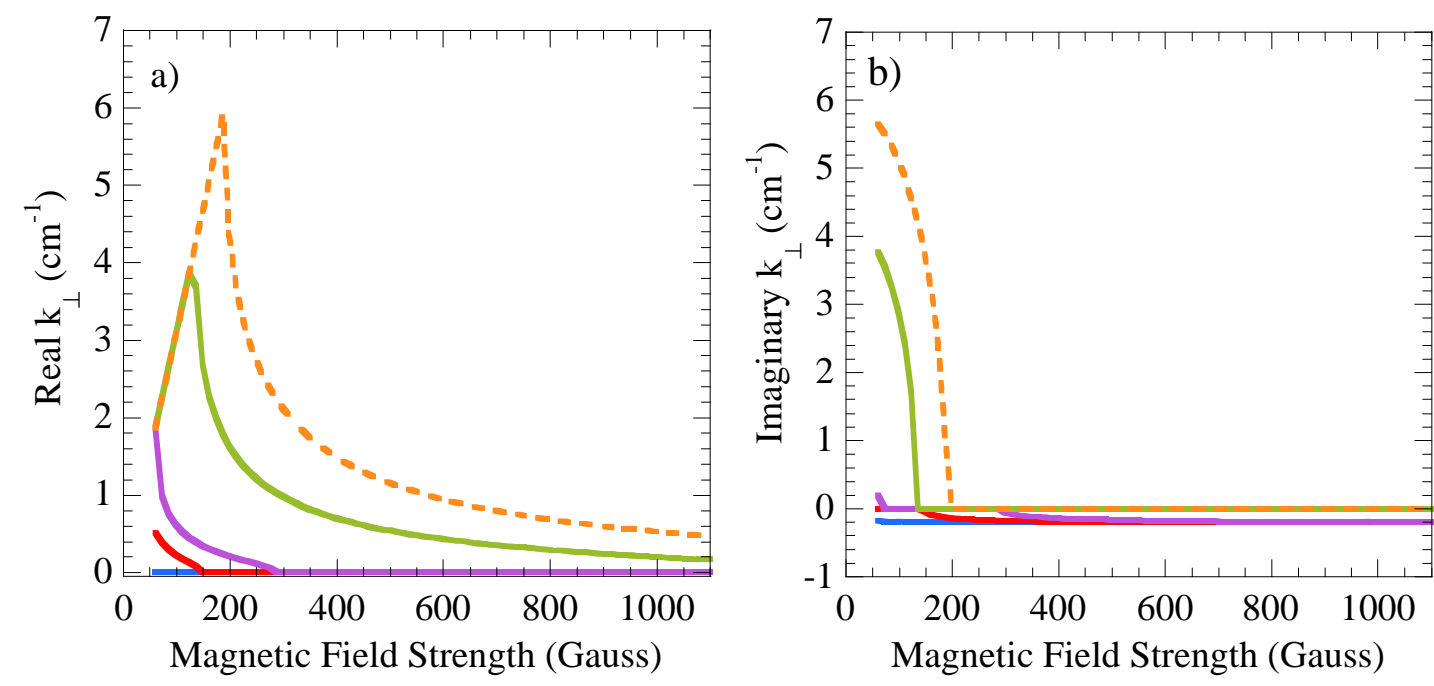

Figure 48: Absolute value of the a) real and b) imaginary components of $\mathrm{k}_{\perp}$ for the fast wave with $k_{\|}=$ $0.26 \mathrm{rad} \mathrm{cm}^{-1}, f=9 \mathrm{MHz}$, no collisions and densities of $(-) n=1 \times 10^{11} \mathrm{~cm}^{-3},(-) n=5 \times 10^{11} \mathrm{~cm}^{-3},(-)$ $n=1 \times 10^{12} \mathrm{~cm}^{-3},(-) n=5 \times 10^{12} \mathrm{~cm}^{-3}$, and $(\ldots .) n=.1 \times 10^{13} \mathrm{~cm}^{-3}$ as a function of magnetic field strength.

Because of the high density, low temperature nature of helicon sources, collisions can play a critical role in wave damping and resonant processes. Typically, the ion-ion and electron-electron collision frequencies are on the order of the RF frequency in helicon sources. When collision are included in Equations (4.15) through (4.17), the resonance in the perpendicular wave number at the lower hybrid frequency resonance for the slow wave becomes finite and the maximum value of both the real and imaginary parts is reduced (Figure 49). Not only are the maximum value of the perpendicular wave numbers reduced, the width of the slow wave resonance is also broadened [Yun and Chang, 1998]. At small magnetic field strengths, collisions reduced the wave numbers 
for the fast wave by 50\% (Figure 50). As the magnetic field strength increases, the effect of collisions on the perpendicular wave number decreases. However, all of the wave numbers are too small for particle-wave interactions with ions.
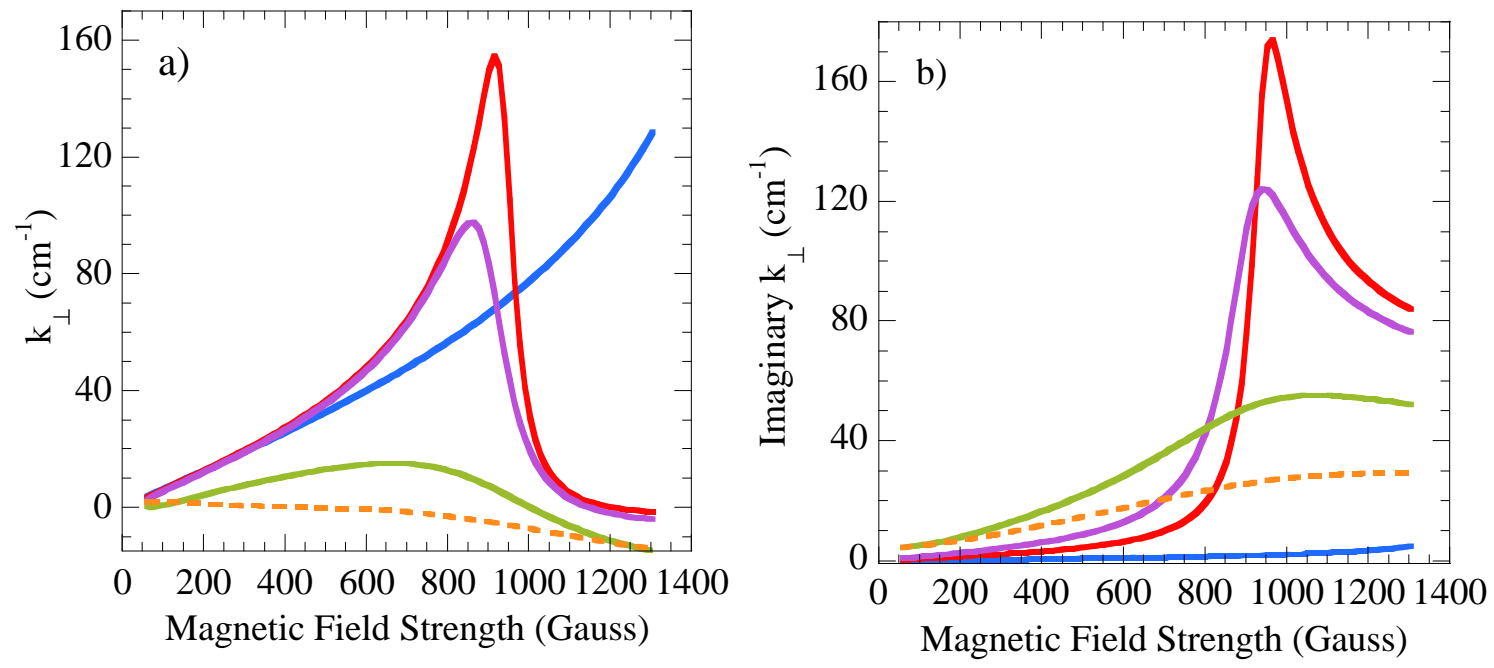

Figure 49: Absolute value of the a) real and b) imaginary components of $\mathrm{k}_{\perp}$ for the slow wave with $k_{\|}=$ $0.26 \mathrm{rad} \mathrm{cm}^{-1}, f=9 \mathrm{MHz}$, neutral pressure of $6.7 \mathrm{mTorr}$, electron temperature of $3 \mathrm{eV}$, ion temperature of $0.2 \mathrm{eV}$ and plasma densities of $(-) n=1 \times 10^{11} \mathrm{~cm}^{-3}$, (一) $n=5 \times 10^{11} \mathrm{~cm}^{-3},(-) n=1 \times 10^{12} \mathrm{~cm}^{-3},(-) n$ $=5 \times 10^{12} \mathrm{~cm}^{-3}$, and $(\cdots \cdots) n=1 \times 10^{13} \mathrm{~cm}^{-3}$ as a function of magnetic field strength.
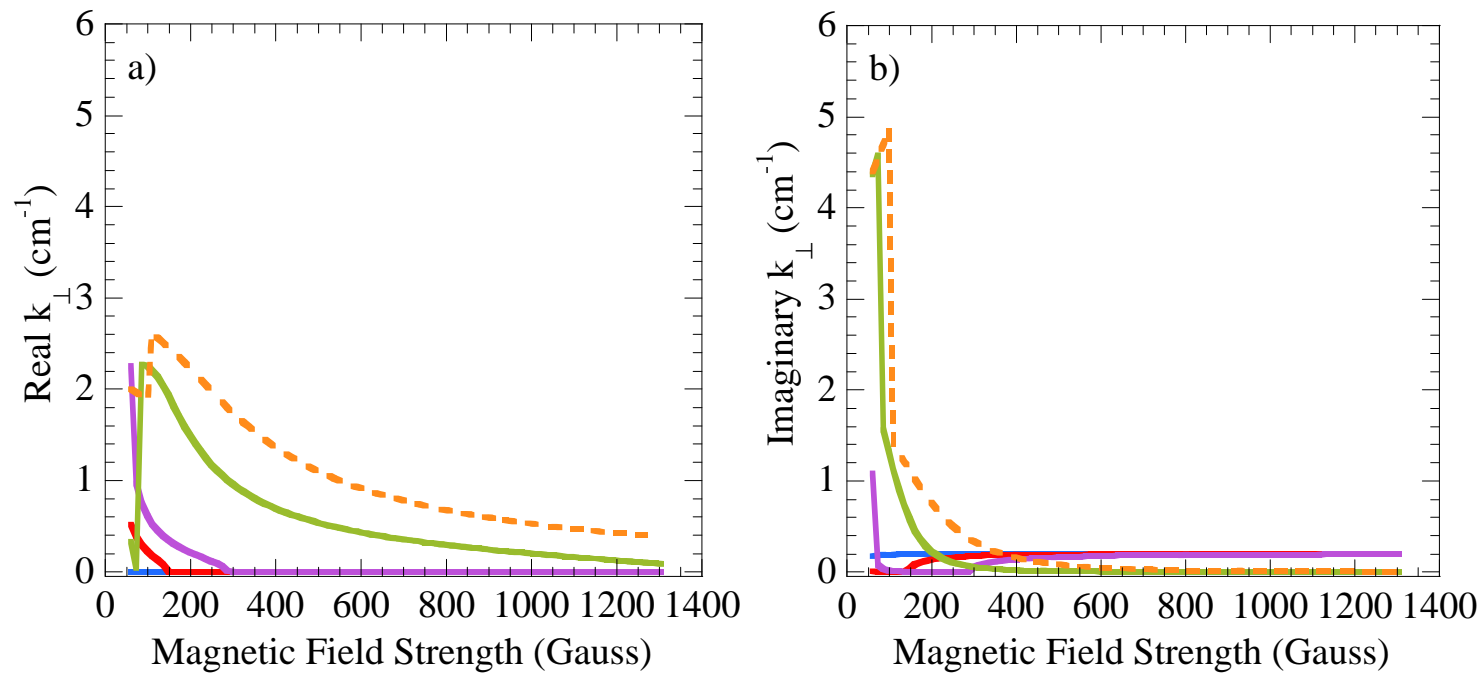

Figure 50: Absolute value of the a) real and b) imaginary components of $k_{\perp}$ for the fast wave with $k_{\|}=$ $0.26 \mathrm{rad} \mathrm{cm}^{-1}, f=9 \mathrm{MHz}$, neutral pressure of $6.7 \mathrm{mTorr}$, electron temperature of $3 \mathrm{eV}$, ion temperature of $0.2 \mathrm{eV}$ and plasma densities of $(-) n=1 \times 10^{11} \mathrm{~cm}^{-3},(-) n=5 \times 10^{11} \mathrm{~cm}^{-3},(-) n=1 \times 10^{12} \mathrm{~cm}^{-3}$, $(-) n=5 \times 10^{12} \mathrm{~cm}^{-3}$, and $(\cdots \cdots) n=1 \times 10^{13} \mathrm{~cm}^{-3}$ as a function of magnetic field strength. 


\subsubsection{Parallel Wave Number Effects on Solutions to the Cold Plasma Dispersion Relation}

The magnitude of the parallel wave number also has a strong effect on the magnitude of the perpendicular wave number for the slow wave required to satisfy the dispersion relation. The calculated perpendicular wave numbers for five different parallel wave numbers, ranging from $k_{\|}=0.01 \mathrm{~cm}^{-1}$ to $k_{\|}=1.0 \mathrm{~cm}^{-1}$, for a plasma density of $5 \mathrm{x} 10^{12}$ $\mathrm{cm}^{-3}$ and RF frequency of $9 \mathrm{MHz}$ are shown in Figure 51a as a function of magnetic field strength. Although the magnetic field at which the lower hybrid resonance occurs remains constant, the magnitudes of both the real and imaginary components of the perpendicular wave number for the slow wave increase with increasing parallel wave number. Arnush's calculations for both the fast and slow waves that included radial and axial boundaries, but not ion motion effects, showed similar increases in the perpendicular wave number for increasing parallel wave number. [Arnush and Chen, 1997]. Balkey reported a sharp increase in edge parallel wave number for operational parameters that yielded the highest ion temperatures in the all Pyrex HELIX experiment [Balkey, 2000]. These calculations suggest that the perpendicular wave number must have also increased for those parameters and therefore the increased ion temperatures could be an indication of Landau damping of waves whose phase velocity had suddenly decreased to values close to the ion thermal speed.
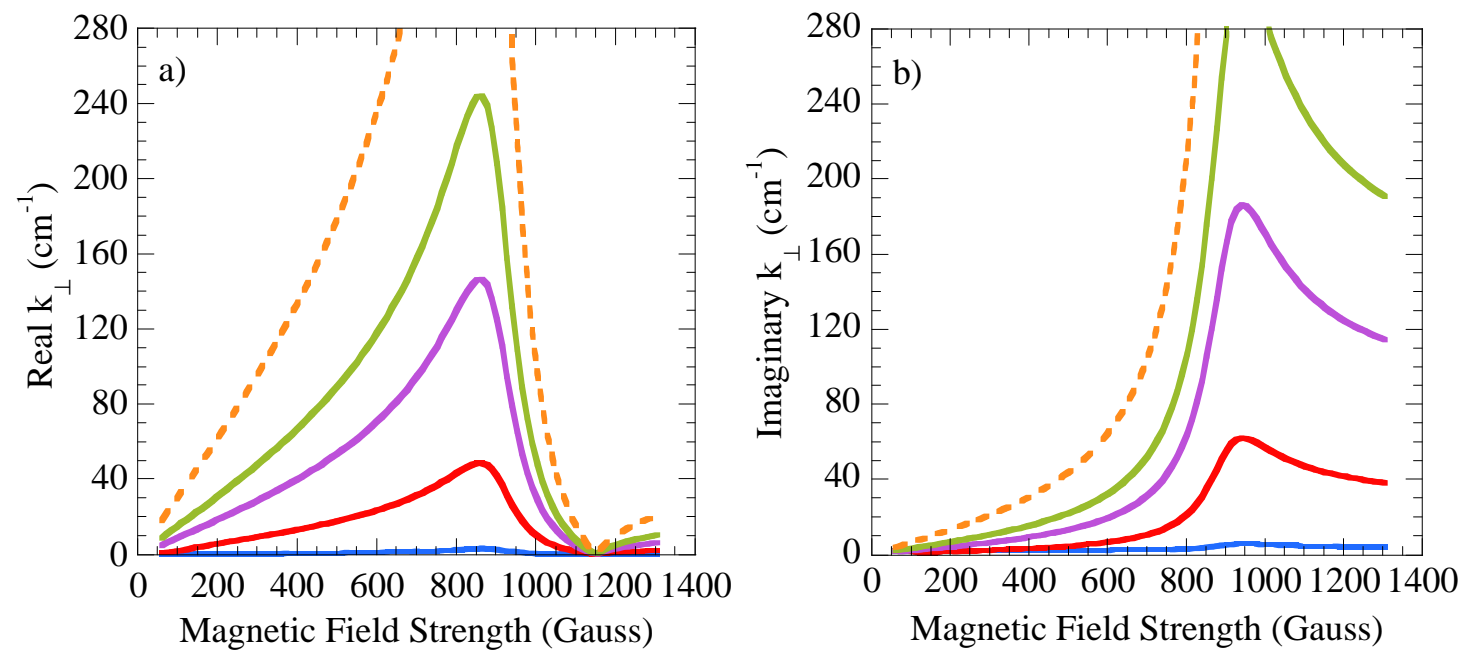

Figure 51: Absolute value of the a) real and b) imaginary components of $k_{\perp}$ for the fast wave verses magnetic field strength with $f=9 \mathrm{MHz}$, density of $5 \times 10^{12} \mathrm{~cm}^{-3}$, neutral pressure of $6.7 \mathrm{mTorr}$, electron temperature of $3 \mathrm{eV}$, ion temperature of $0.2 \mathrm{eV}$, and parallel wave numbers of $(-) k_{\|}=0.01 \mathrm{rad} \mathrm{cm}^{-1},(-)$ $k_{\|}=0.1 \mathrm{rad} \mathrm{cm}^{-1},\left({ } k_{\|}=0.3 \mathrm{rad} \mathrm{cm}^{-1},(-) k_{\|}=0.5 \mathrm{rad} \mathrm{cm}^{-1}\right.$, and $(\cdots \cdots) k_{\|}=1.0 \mathrm{rad} \mathrm{cm}^{-1}$. 


\subsubsection{Wave Frequency Effects on Solutions to the Cold Plasma Dispersion Relation}

The real and imaginary components of the perpendicular wave number for the slow wave are shown in Figure 52 as a function of magnetic field strength for a range of RF frequencies. As expected for a plasma density of $5 \times 10^{12} \mathrm{~cm}^{-3}$, the lower hybrid resonance shifts to higher magnetic field strengths for higher RF frequencies. The higher magnetic field strengths increase the lower hybrid frequency to match the RF frequency. The maximum value of the perpendicular wave number also increases with increasing RF frequency for a fixed choice of $k_{\|}$and plasma density.
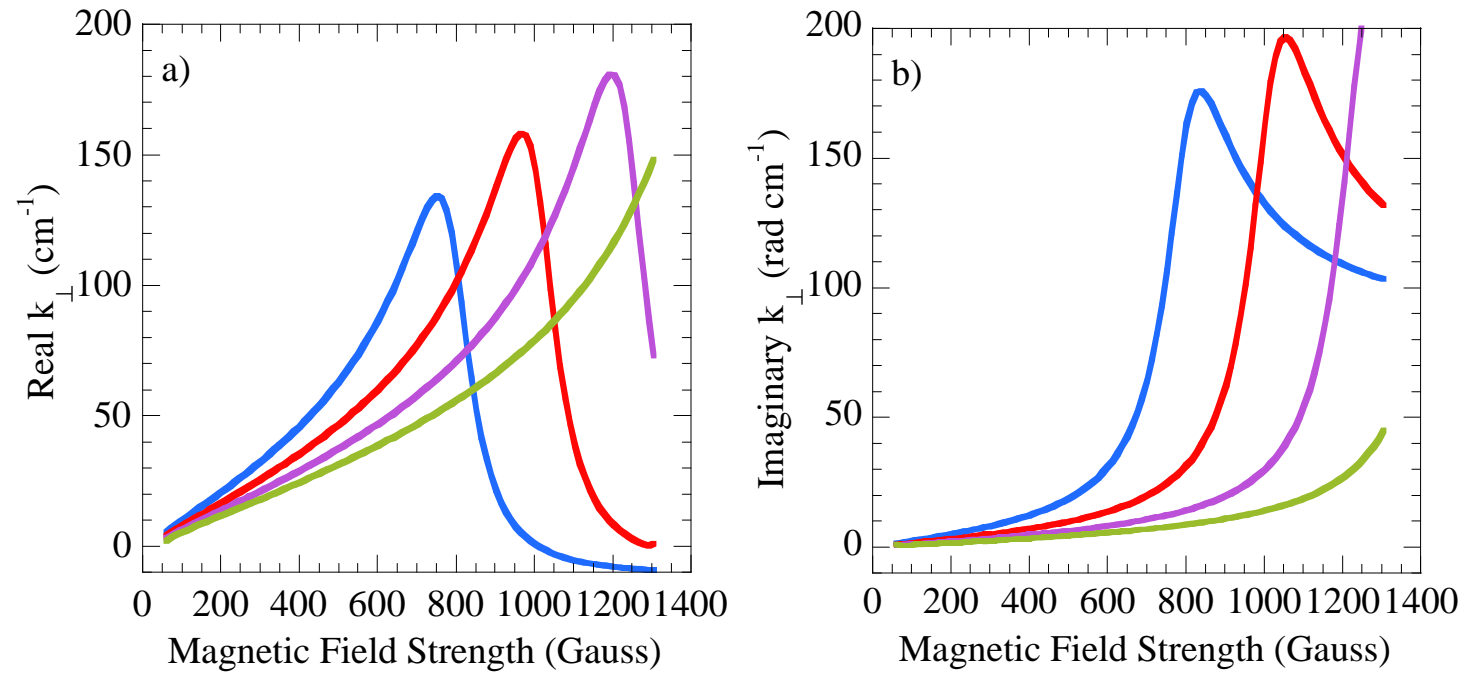

Figure 52: Absolute value of the a) real and b) imaginary components of $k_{\perp}$ for the slow wave verses magnetic field strength with $k_{\|}=0.26$, density of $5 \times 10^{12} \mathrm{~cm}^{-3}$, neutral pressure of $6.7 \mathrm{mTorr}$, electron temperature of $3 \mathrm{eV}$, ion temperature of $0.2 \mathrm{eV}$ and driving frequencies of $(-) f=8 \mathrm{MHz},(-) f=10$ $\mathrm{MHz},(-) f=12 \mathrm{MHz}$, and $(-) f=14 \mathrm{MHz}$.

For the fast wave, increases in the RF frequency increase the perpendicular wave number according to Equation (4.7) for a fixed plasma density and parallel wave number. Initial HELIX experiments found that increases in the RF frequency lead to reductions in the plasma density that appeared consistent with Equation (4.7) assuming the wave number remained fixed [Keiter et al., 1997]. Later experiments demonstrated that the inverse dependence of the plasma density on RF frequency only occurred for a narrow range of magnetic fields (well away from the lower hybrid resonance) [Balkey, 2000]. The anomalous behavior of the plasma density during operation near the lower hybrid resonance was an early indication that damping of the helicon wave (via collisions or 
Landau damping) could not be responsible for the coupling the RF power into HELIX plasmas.

\subsubsection{The Cold Plasma Computational Model for Arbitrary Density Profiles}

For a typical plasma in HELIX, the only plasma parameter that changes significantly with radius is the plasma density, i.e., the ionization fraction of the neutral gas. In fact, the slow and fast wave dispersion relations should change so dramatically with radius it is unreasonable to expect that dispersion relations derived for homogeneous plasmas can accurately describe helicon source dynamics. The fast wave cannot even propagate in the edge of a typical helicon source because the wavelength at the edge is longer than the plasma chamber.

For the fast wave, the important scale size is the electron gyroradius as ion motion effects are ignorable. Even though ion motion plays an important role in the resonant behavior of the slow wave, the large perpendicular wave numbers of the slow wave imply that the ions are unmagnetized and the slow wave is primarily an electron wave [Cho, 2000]. Thus, if the scale length of the density gradient, $n / \nabla n$, is much larger than the electron gyroradius, a helicon source plasma can be divided into a series of concentric plasma "annuli," each with a different plasma density. The cold plasma dispersion relationship is then solved for the plasma parameters, e.g., density, temperature, and collision frequency, at each radial location. The true of effect of the radial boundary conditions, conducting or insulating, and antenna geometry is missing from such a crude model. However, because the plasma parameters vary so much from edge to center in a helicon source, the rapidly changing dispersive properties of the plasma medium are likely to play a greater role in governing wave propagation, particularly downstream from the antenna, than the distant boundaries. Near the plasma edge and near the antenna, the antenna currents and boundary conditions are critically important and in that region, an "eigenmode" type model that includes coupling effects at the boundary [Cho, 2000] would provide a better representation of the wave fields and antenna loading.

Using the discrete plasma annuli model of the helicon source, a second MATLAB program was written for arbitrary density profiles. A root finding algorithm solves the cold plasma dispersion relationship for a series of plasma density and collision frequency 
values for fixed values of the parallel wave number, electron temperature, and ion temperature. The plasma densities and collision frequencies are calculated from a user defined plasma density profile. The user defined density profiles are all of the form

$$
N(r)=n_{o}\left(1-(r / a)^{b}\right)^{c}
$$

where $N$ is the density as a function of radius, $n_{o}$ is the peak density on the magnetic field axis, $b$ and $c$ are factors for changing the shape of the profile, and $a$ is the plasma radius. The calculated collision frequencies include: ion-ion, electron-electron, ion-neutral, and electron-neutral collisions. The ion-neutral and electron-neutral collision frequency calculations require a user supplied value for the edge neutral pressure. The program also includes an option to let the plasma density profile and peak density change with magnetic field strength in accordance with experimentally measured density profiles at each magnetic field strength. A copy of the MATLAB codes can be found in Appendix A. The calculated real and imaginary components of the perpendicular wave number are then plotted as a function of radius and magnetic field strength for a given RF frequency.

a)

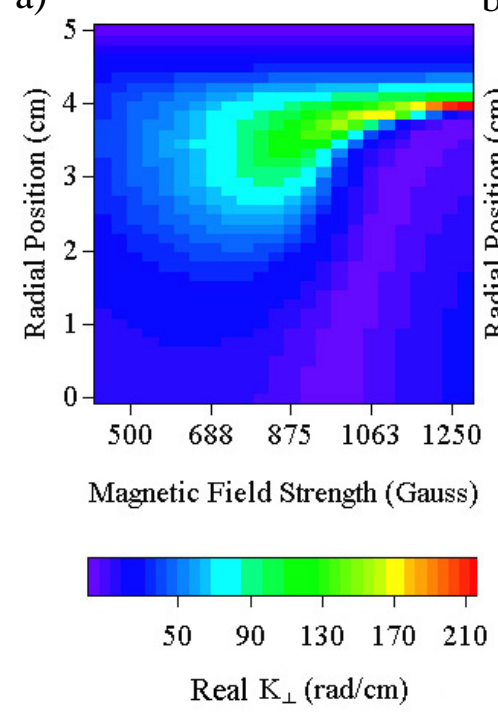

b)
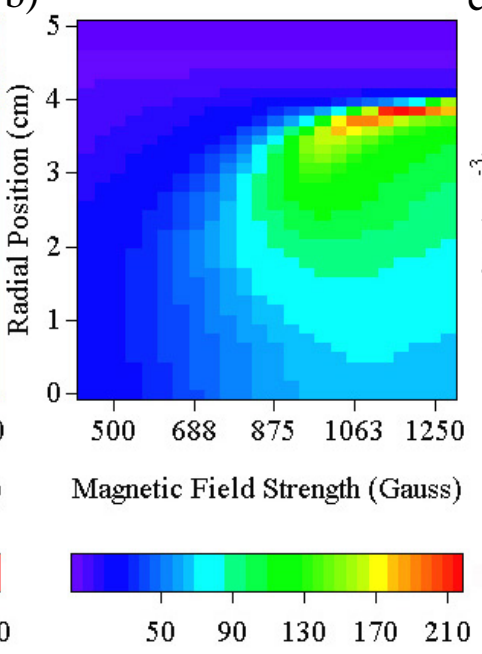

Imaginary $\mathrm{K}_{\perp}(\mathrm{rad} / \mathrm{cm})$ c)

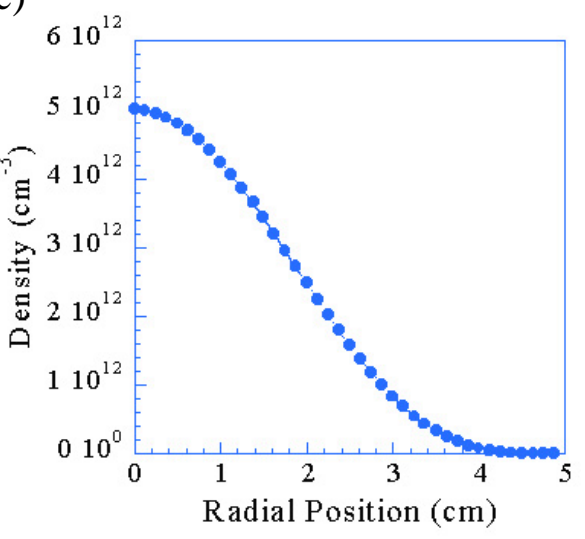

Figure 53: Absolute value of the a) real and b) imaginary components of $k_{\perp}$ for the slow wave as a function of radius and magnetic field strength with $k_{\|}=0.3 \mathrm{rad} \mathrm{cm}^{-1}$, neutral pressure of $6.7 \mathrm{mTorr}$, electron temperature of $3 \mathrm{eV}$, ion temperature of $0.2 \mathrm{eV}$ and $f=9 \mathrm{MHz}$ for the given c) density profile.

An example calculation for a parabolic density profile with a peak density of $5 \times 10^{12}$ $\mathrm{cm}^{-3}$, an RF frequency of $9 \mathrm{MHz}$, an electron temperature of $3 \mathrm{eV}$, an ion temperature of 
$0.2 \mathrm{eV}$, a neutral pressure of $6.7 \mathrm{mTorr}$, and a parallel wave number of $0.3 \mathrm{rad} / \mathrm{cm}$ is shown in Figure 53. The real and imaginary parts of the perpendicular wave number for the slow wave are shown as functions of radius and magnetic field strength in Figure 53a and Figure 53b respectively. The density profile used for all magnetic field strengths in the calculation is shown in Figure 53c. At high magnetic field strengths, a clear resonance layer appears in the plasma edge. The real perpendicular wave numbers rise from the edge of the plasma until they reach a maximum and then decrease sharply. Inward of the maximum real wave number, the imaginary component of the wave number reach a maximum; indicating strong damping of the slow wave as is expected for a surface wave [Shamrai and Taranov, 1996]. The resonance layer is narrowest at the high magnetic fields and vanishes completely at the lowest magnetic fields $(B<700 \mathrm{G})$.

Figure 54a and Figure 54b show the real and imaginary components of the perpendicular wave number for the slow wave as a function of radius and magnetic field strength and the experimentally measured density profiles at each value of magnetic field strength (Figure 54c). For each of the calculations, an RF frequency of $9 \mathrm{MHz}$, an electron temperature of $3 \mathrm{eV}$, an ion temperature of $0.2 \mathrm{eV}$, a neutral pressure of 6.7 mTorr, and a parallel wave number of $0.3 \mathrm{rad} / \mathrm{cm}$ were used. Note that the resolution in both radius and magnetic field strength is lower than in the previous example, but the same trends in the real and imaginary components are evident. A resonance layer appears at the plasma edge at high magnetic field strengths and moves outward as the magnetic field strength increases. The real component of the perpendicular wave number peaks at larger radii than the imaginary component.

These two examples are representative of the changes in the perpendicular wave number components as a function of radius and magnetic field. The exact location of the resonance layer is sensitive to the details of the density profile, but the resonance layer is always close to the edge of the plasma. In all cases, the calculations predict that the slow wave will be strongly damped at the plasma edge above some critical value of magnetic field strength. 


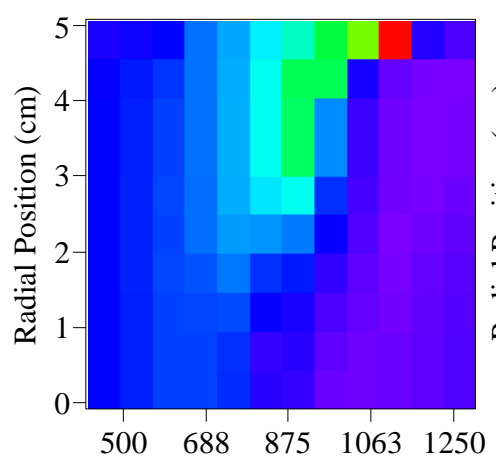

Magnetic Field Strength (Gauss)

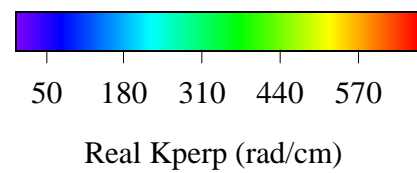

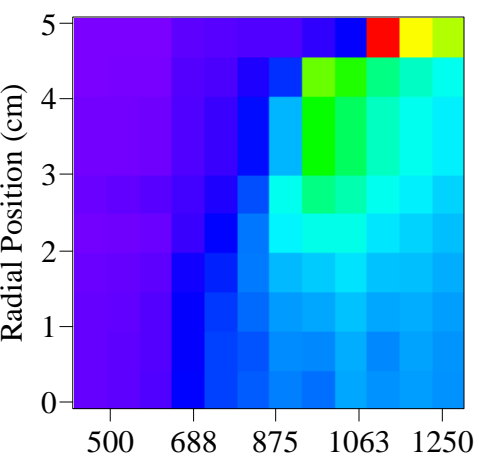

Magnetic Field Strength (Gauss)

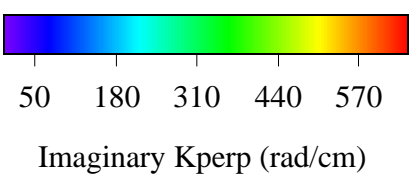

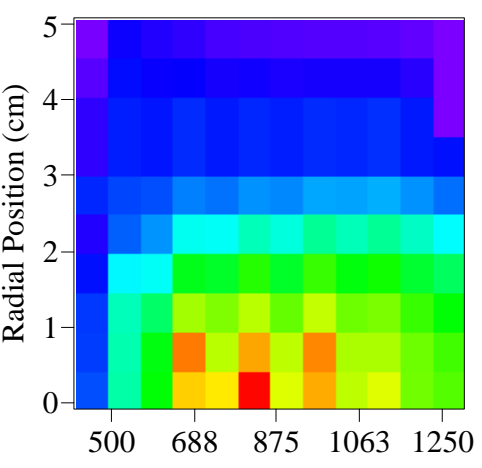

Magnetic Field Strength (Gauss)

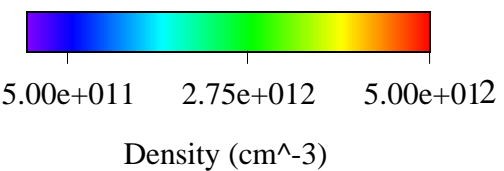

Figure 54: Absolute value of the a) real and b) imaginary components of $k_{\perp}$ for the slow wave as a function of radius and magnetic field strength with $k_{\|}=0.3 \mathrm{rad} \mathrm{cm}^{-1}$, neutral pressure of $6.7 \mathrm{mTorr}$, electron temperature of $3 \mathrm{eV}$, ion temperature of $0.2 \mathrm{eV}$ and $f=9 \mathrm{MHz}$ for the given c) experimentally measured density profile.

\subsubsection{Maximum Values of $k_{\perp}$ for the Slow Wave as a Function of RF Frequency and Magnetic Field Strength}

From the plots in the previous section, it is clear that at high magnetic field strengths there is a point at some radius near the plasma edge where the real part of the perpendicular wave number is maximum. As the density increases with decreasing radius, the real part of the wave number peaks before the imaginary part of the wave number peaks. Although the calculated location of resonance suggests a possible region to study with probes or the radially scanning LIF system, the simple existence of large real components of the perpendicular wave number implies that ion Landau damping of low phase velocity slow waves (TG modes) can take place in the helicon source plasma. To determine for what RF frequencies and magnetic field strengths ion Landau damping could occur somewhere in HELIX, the MATLAB ${ }^{\mathrm{TM}}$ code was modified to store the maximum (at any radius) real and imaginary components of the perpendicular wave number for an array of RF frequency and magnetic field strength values. Plots of maximum real and imaginary components of the perpendicular wave number as a function of RF frequency and magnetic field strength could then be compared with plots 
of perpendicular ion temperature as a function of RF frequency and magnetic field strength to determine if the parameters yielding the hottest ions should also yield the largest perpendicular wave numbers.

Examples of such calculations are shown in Figure 55 and Figure 56. The normalized real perpendicular wave numbers for the slow wave, $k_{\perp} v_{t h i} / \omega$, are shown in Figure 55a for magnetic fields of $250-3300$ Gauss and for RF frequencies from 5 to $30 \mathrm{MHz}$. Figure $55 \mathrm{~b}$ is an enlargement of the portion of Figure 55a that includes the operating range of HELIX. Figure 56a and Figure 56b show the imaginary slow wave wave numbers for the same parameters as in Figure 55. For these calculations, a peak plasma density of $5 \mathrm{x}$ $10^{12} \mathrm{~cm}^{-3}$, an electron temperature of $3 \mathrm{eV}$, an ion temperature of $0.2 \mathrm{eV}$, a neutral pressure of $6.7 \mathrm{mTorr}$, and a parallel wave number of $0.3 \mathrm{rad} / \mathrm{cm}$ were used

The decrease in the lower hybrid frequency due to the ion plasma frequency term is responsible for the asymptotic behavior of the lower hybrid resonance curve in Figure 55a (the yellow and orange regions in Figure 55a). If the ion plasma frequency term was ignorable, the RF frequency at which the peak in the perpendicular wave number occurred would increase linearly with increasing magnetic field strength. The expected linear relationship is evident for low magnetic field strength and low RF frequency in Figure 57a for which the peak plasma density was increased from $5 \times 10^{12} \mathrm{~cm}^{-3}$ to $2 \mathrm{x}$ $10^{13} \mathrm{~cm}^{-3}$. At high magnetic field strength and high RF frequency, the lower hybrid resonance begins to curve gently due to the increased role of the ion plasma frequency term.

A key result predicted by these two calculations is that for a helicon source operating at $27 \mathrm{MHz}$ and roughly $1 \mathrm{kGauss,}$, ion heating due to Landau damping of slow waves cannot be achieved if the density is constant because the lower hybrid frequency is essentially the ion plasma frequency, $\omega_{l h} \sim \omega_{p i}$, that only depends on the plasma density. For helicon sources using argon that operate in the $7-30 \mathrm{MHz}$ and $400-3000$ Gauss range, the lower hybrid frequency has a strong dependence on the plasma density as well as magnetic field strength for densities less than $1 \times 10^{13} \mathrm{~cm}^{-3}$. For densities greater than $1 \times 10^{13} \mathrm{~cm}^{-3}$, the lower hybrid frequency primarly depends on the magnetic field strength. For processes that depend on the lower hybrid frequency, these are two different regimes of operation. 
Figure 58a, and Figure 58b are plots of the imaginary component of the perpendicular wave number for the same parameters as Figure 57. The imaginary component has multiple bands where the imaginary wave numbers peak. The physical nature of the bands is not understood. As for the damping rate in the HELIX operation range, it is clear that the damping is smaller at larger densities.

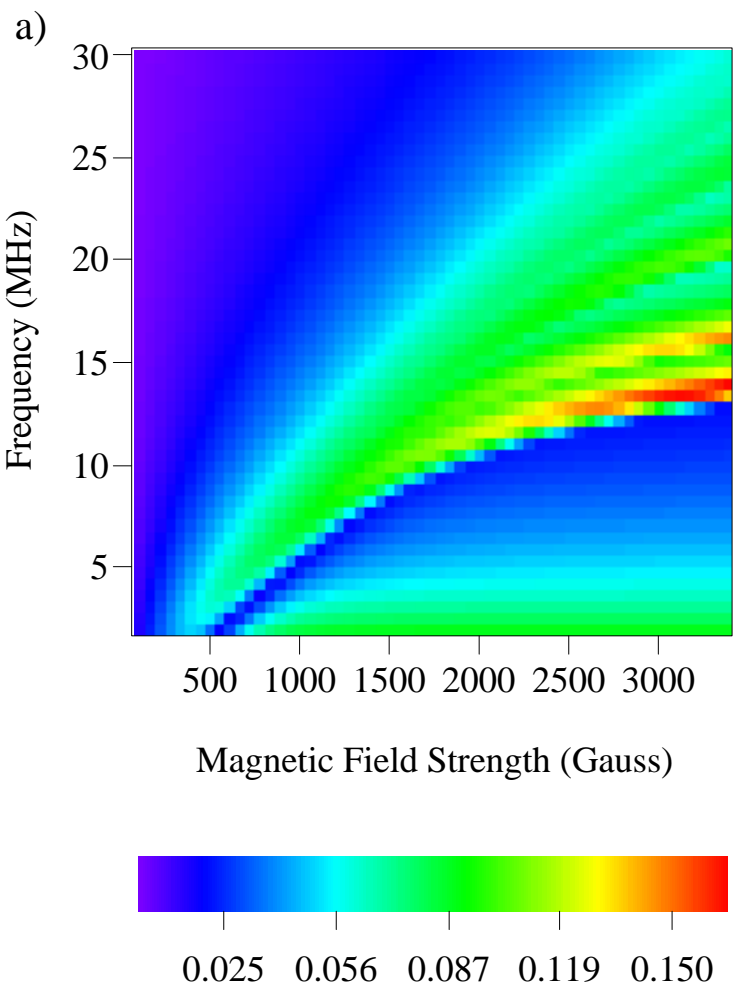

Normalized Wave Numbers $\left(\mathrm{V}_{\text {thi }} k_{\perp} / \omega\right)$
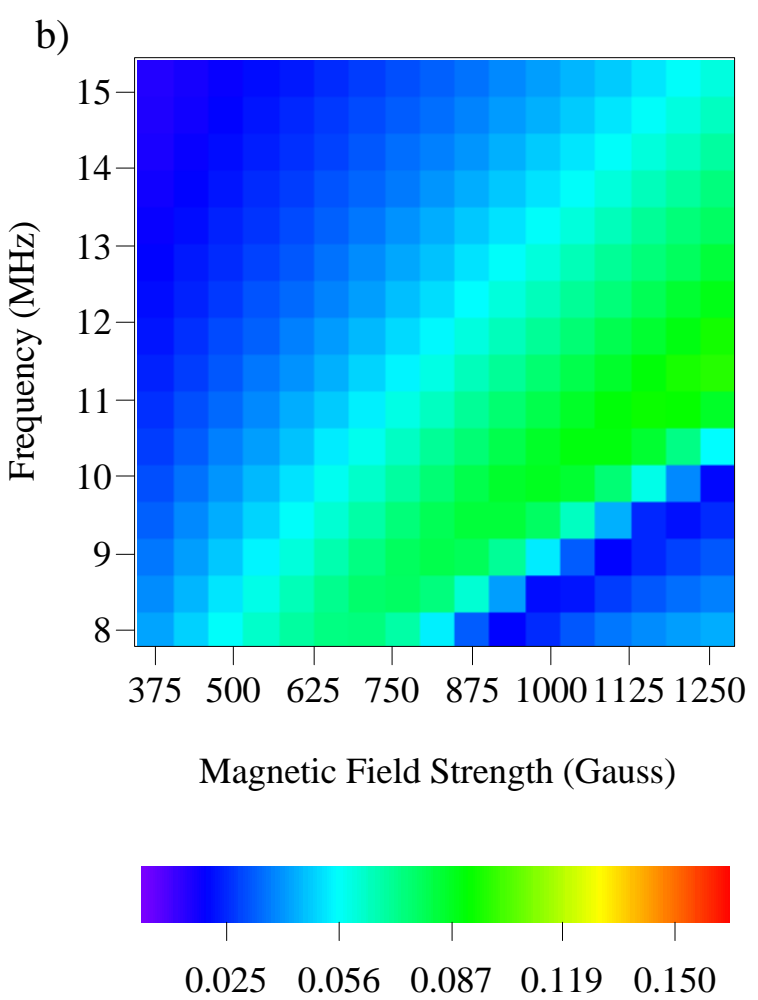

Normalized Wave Numbers $\left(\mathrm{V}_{\text {thi }} k_{\perp} / \omega\right)$

Figure 55: a) Maximum value of the real component of $k_{\perp}$ for the slow wave as a function of RF driving frequency and magnetic field strength for a peak density of $5 \times 10^{12} \mathrm{~cm}^{-3}$, a neutral pressure of $6.7 \mathrm{mTorr}$, electron temperature of $3 \mathrm{eV}$, ion temperature of $0.2 \mathrm{eV}$, and $\mathrm{k}_{\|}$of $0.3 \mathrm{rad} \mathrm{cm}^{-1}$. b) Enlargement of (a) for the operating parameters of HELIX. 
a)

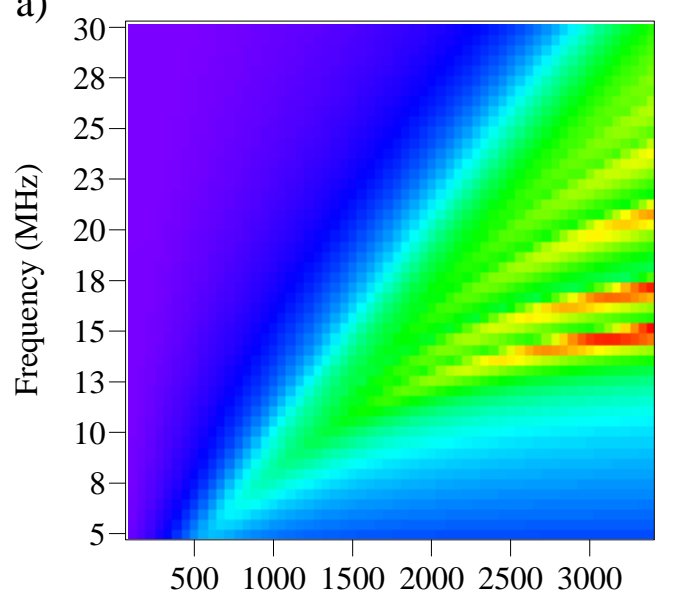

Magnetic Field Strength (Gauss)

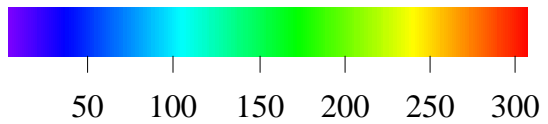

b)

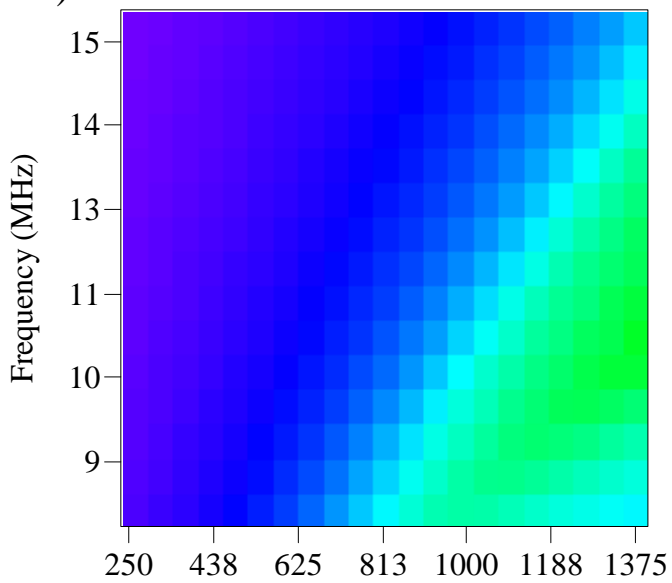

Magnetic Field Strength (Gauss)

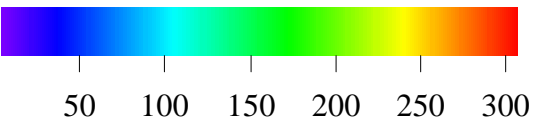

Aaximum Imaginary Wave Numbers $(\mathrm{rad} / \mathrm{cm})$

Maximum Imaginary Wave Numbers $(\mathrm{rad} / \mathrm{cm})$

Figure 56: a) Maximum value of the imaginary component of $k_{\perp}$ for the slow wave as a function of RF driving frequency and magnetic field strength for a peak density of $5 \times 10^{12} \mathrm{~cm}^{-3}$, a neutral pressure of 6.7 mTorr, electron temperature of $3 \mathrm{eV}$, ion temperature of $0.2 \mathrm{eV}$, and $k_{\|}$of $0.3 \mathrm{rad} \mathrm{cm}^{-1}$. b) Enlargement of (a) for the operating parameters of HELIX.

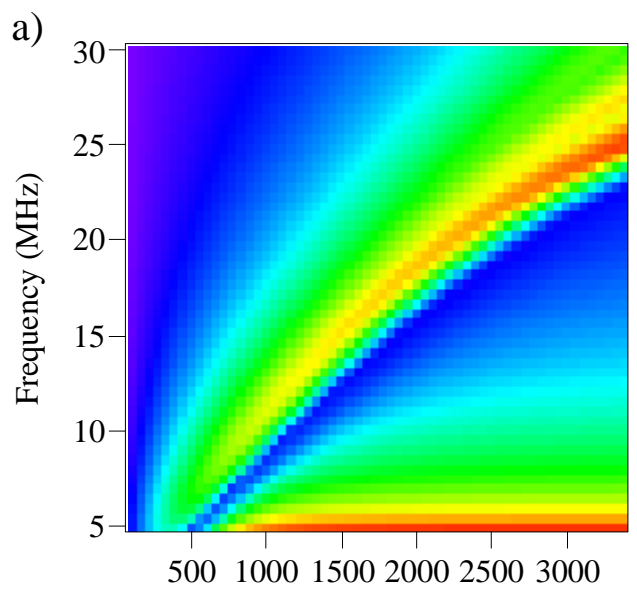

Magnetic Field Strength (Gauss)

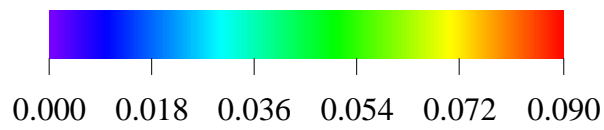

Normalized Wave Number $\left(\mathrm{v}_{\text {thi }} k_{\perp} / \omega\right)$ b)

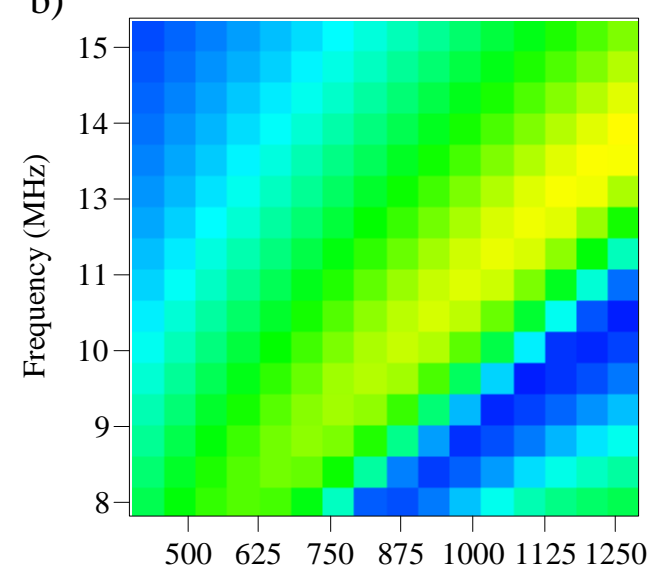

Magnetic Field Strength (Gauss)

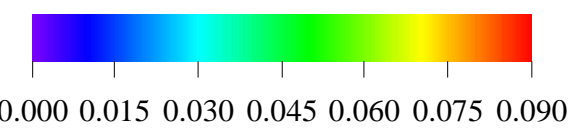

Normalized Wave Numbers $\left(\mathrm{v}_{\text {thi }} k_{\perp} / \omega\right)$

Figure 57: a) Maximum value of the real component of $k_{\perp}$ for the slow wave as a function of RF driving frequency and magnetic field strength for a peak density of $2 \times 10^{13} \mathrm{~cm}^{-3}$, a neutral pressure of $6.7 \mathrm{mTorr}$, electron temperature of $3 \mathrm{eV}$, ion temperature of $0.2 \mathrm{eV}$, and $k_{\|}$of $0.3 \mathrm{rad} \mathrm{cm}^{-1}$. b) Enlargement of (a) for the operating parameters of HELIX. 
a)

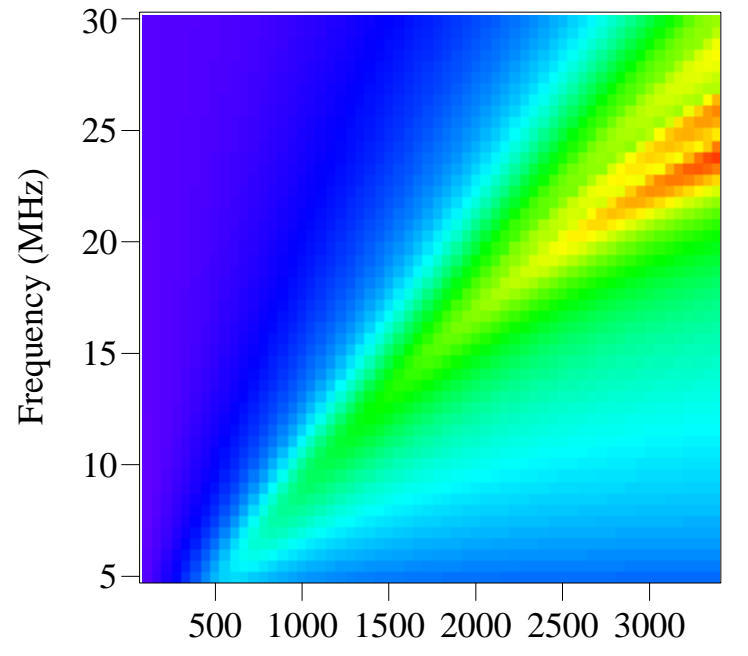

Magnetic Field Strength (Gauss)

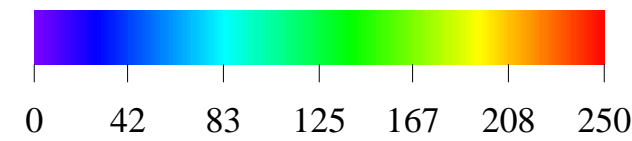

b)

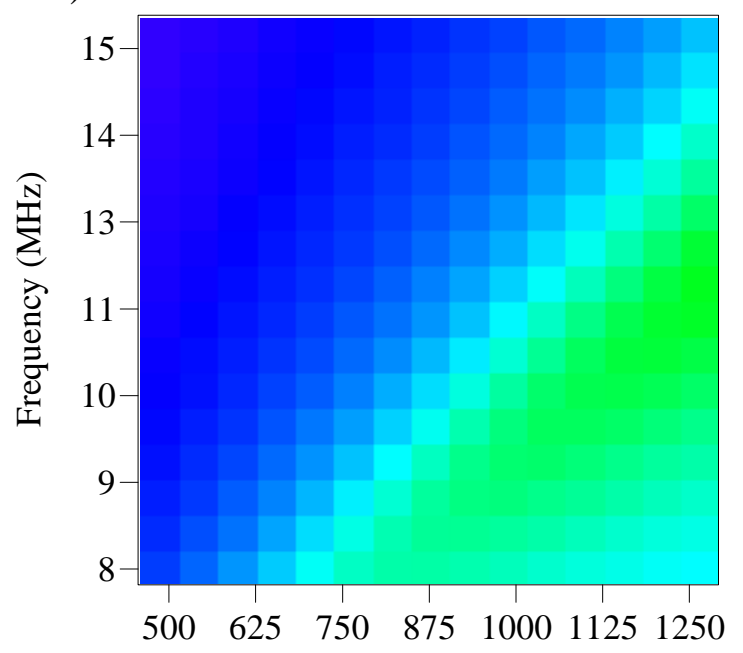

Magnetic Field Strength (Gauss)

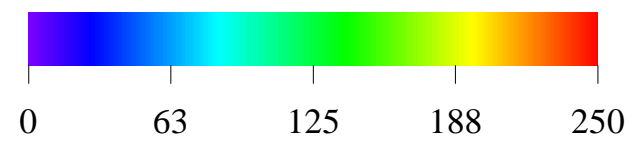

Maximum Imaginary Wave Numbers ( $\mathrm{rad} / \mathrm{cm}) \quad$ Maximum Imaginary Wave Numbers $(\mathrm{rad} / \mathrm{cm})$

Figure 58: a) Maximum value of the imaginary component of $k_{\perp}$ for the slow wave as a function of RF driving frequency and magnetic field strength for a peak density of $2 \times 10^{13} \mathrm{~cm}^{-3}$, a neutral pressure of 6.7 mTorr, electron temperature of $3 \mathrm{eV}$, ion temperature of $0.2 \mathrm{eV}$, and $k_{\|}$of $0.3 \mathrm{rad} \mathrm{cm}^{-1}$. b) Enlargement of (a) for the operating parameters of HELIX 


\section{Chapter 4 References}

Arnush, D., The role of Trivelpiece-Gould waves in antenna coupling to helicon waves, Phys. Plasmas, 7, 3042 (2000).

Arnush, D., and Chen, F.F., Generalized theory of helicon waves. I. Normal modes., Phys. Plasmas, 4, 3411 (1997).

Balkey, M.M., Optimization of a Helicon Plasma Source for Maximum Density with Minimal Ion Heating, Ph. D. thesis, West Virginia University, Morgantown (2000).

Balkey, M.M., Boivin, R.F., Kline, J.L., and Scime, E.E., Ion heating and density production in helicon sources near the lower hybrid frequency, Plasma Sources Sci. Technol., 10, 284 (2001).

Blackwell, B.D., Madziwa, M.D., Arnush, D., and Chen, F.F., Direct Detection of Trivelpiece-Gould Modes in Helicon Discharges, Bulletin of the American Physical Society, 46, 63 (2001).

Blevin, H.A., and Christiansen, P.J., Austrailian Journal of Physics, 15, 501 (1966).

Boivin, R.F., Kline, J.L., and Scime, E.E., Electron temperature measurement by a helium line intensity ratio method in helicon plasmas, Phys. Plasmas, 8, 5303 (2001).

Borg, G.G., and Boswell, R.W., Power coupling to helicon and Trivelpiece-Gould modes in helicon sources, Phys. Plasmas, 5, 564 (1998).

Boswell, R.W., Plasma production using a standing helicon wave, Phys. Lett. A, 33, 457 (1970).

Boswell, R.W., Dependence of helicon wave radial structure on electron inertia, Aus. $J$. Phys., 25, 403 (1972).

Boswell, R.W., Very efficient plasma generation by whistler waves near the lower hybrid frequency, Plasma Phys. Controlled Fusion, 26, 1147 (1984).

Boswell, R.W., and Porteous, R.K., Large volume, high density RF inductively coupled plasma, Appl. Phys. Lett., 50, 1130 (1987).

Boswell, R.W., and Vender, D., An experimental study of breakdown in a pulsed Helicon plasma, Plasma Sources Sci. Technol., 4, 534 (1995).

Chang Diaz, F.R., Goulding, R.H., Bengtson, R.D., Wally Baity, F., Sparks, D., Bussell, R.G., Jr., Barber, G.C., McCaskill, G., Jacobson, V.T., Carter, M.D., Ilin, A.V., and Glover, T.W., Experimental status of the development of a variable specific impulse magnetoplasma rocket, Fusion Technology, 35, 243 (1999).

Chen, F.F., Experiments on helicon plasma sources, J. Vac. Sci. Technol. A, 10, 1389 (1992).

Chen, F.F., and Arnush, D., Generalized theory of helicon waves. I. Normal modes, Phys. Plasmas, 4, 3411 (1997).

Chen, F.F., and Decker, C.D., Electron acceleration in helicon sources, Plasma Phys. Controlled Fusion, 34, 635 (1992).

Chen, F.F., Hsieh, M.J., and Light, M., Helicon waves in a non-uniform plasma, Plasma Sources Sci. Technol., 3, 49 (1994).

Chen, F.F., Jiang, X., and Evans, J.D., Plasma injection with helicon sources, J. Vac. Sci. Technol. A, 18, 2108 (2000). 
Chen, F.F., Jiang, X., Evans, J.D., Tynan, G., and Arnush, D., Low-field helicon discharges, Plasma Phys. Controlled Fusion, 39, 411 (1997).

Chi, K.K., Sheridan, T.E., and Boswell, R.W., Resonant cavity modes of a bounded helicon discharge, Plasma Sources Sci. Technol., 8, 421 (1999).

Cho, S., The field and power absorption profiles in helicon plasma resonators, Phys. Plasmas, 3, 4268 (1996).

Cho, S., The role of the lower hybrid resonance in helicon plasmas, Phys. Plasmas, 7, 417 (2000).

Cho, S., and Kwak, J.G., The effects of the density profile on the power absorption and the equilibrium density in helicon plasmas, Phys. Plasmas, 4, 4167 (1997).

Cohen, S.A., Berlinger, B., Corso, V., Fahmy, F., Gorman, J., Levinton, F., Lemunyan, G., and Fredriksen, A., The magnetic nozzle experiment, Bulletin of the American Physical Society, 44 (1999).

Davies, B., Helicon wave propagation: effect of electron inertia, J. Plasma Phys., 4, 43 (1970).

Degeling, A., Mikhelson, N., Boswell, R., and Sadeghi, N., Characterization of helicon waves in a magnetized inductive discharge, Phys. Plasmas, 5, 572 (1998).

Degeling, A.W., Jung, C.O., Boswell, R.W., and Eliingboe, A.R., Plasma production from helicon waves, Phys. Plasmas, 3, 2788 (1996).

Durandet, A., Davis, C.A., and Boswell, R.W., Pure silicon plasma in a helicon plasma deposition system, Appl. Phys. Lett., 70, 1814 (1997).

Franck, C., and Klinger, T., Helicons - Our Last Year, Bulletin of the American Physical Society, 46 (2000).

Gilland, J., Breun, R., and Hershkowitz, N., Neutral pumping in a helicon discharge, Plasma Sources Sci. Technol., 7, 416 (1998).

Greenberg, K.E., Hargis, P.J., and Miller, P.A., The GEC RF Reference Cell: Diagnostic techniques and initial results, SETEC Report 90-013 (1990).

Grulke, O., Greiner, F., Klinger, T., and Piel, A., Comparative experimental study of coherent structures in a simple magnetized torus, Plasma Phys. Controlled Fusion, 43, 525 (2001).

Guo, X.M., Scharer, J., Mouzouris, Y., and Louis, L., Helicon experiments and simulations in nonuniform magnetic field configurations, Phys. Plasmas, 6, 3400 (1999).

Hanna, J., and Watts, C., Alfvén wave propagation in a helicon plasma, Phys. Plasmas, 8 , 4251 (2001).

Hershkowitz, N., Ding, J., Breun, R.A., Chen, R.T.S., Meyer, J., and Quick, A.K., Does high density-low pressure etching depend on the type of plasma source?, Phys. Plasmas, 3, 2197 (1996).

Keiter, P.A., Experimental Investigation of Ion Temperature Anisotropy Driven Instabilities in a High Beta Plasma, Ph. D. thesis, West Virginia University, Morgantown (1999).

Keiter, P.A., Scime, E.E., and Balkey, M.M., Frequency dependent effects in helicon plasmas, Phys. Plasmas, 4, 2741 (1997).

Keiter, P.A., Scime, E.E., Balkey, M.M., Boivin, R., Kline, J.L., and Gary, S.P., Betadependent upper bound on ion temperature anisotropy in a laboratory plasma, Phys. Plasmas, 7, 779 (2000). 
Kline, J.L., Scime, E.E., Boivin, R.F., Keesse, A., and Sun, X., RF Absoption and Ion Heating in Helicon Sources, to appear in Phys. Rev. Lett. (2002).

Kwak, J.G., Choi, H.D., Bak, H.I., Suwon, C., Bak, J.G., and Kim, S.K., Frequency dependence of the plasma density for helicon plasmas, Phys. Plasmas, 4, 1463 (1997).

Kwak, J.G., Kim, S.K., and Cho, S., Upper limit to the monotonic increasing dependence of the plasma density on the magnetic field in helicon discharges, Phys. Lett. A, 267, 384 (2000).

Lho, T., Hershkowitz, N., Miller, J., Steer, W., and Kim, G.H., Azimuthally symmetric pseudosurface and helicon wave propagation in an inductively coupled plasma at low magnetic field, Phys. Plasmas, 5, 3135 (1998).

Light, M., Chen, F.F., and Colestock, P.L., Low frequency electrostatic instability in a helicon plasma, Phys. Plasmas, 8, 4675 (2001).

Light, M., Sudit, I.D., Chen, F.F., and Arnush, D., Axial propagation of helicon waves, Phys. Plasmas, 2, 4094 (1995).

Light, M.E., A study of Low Frequency Instability and Transport in a Helicon Plasma, Ph. D. thesis, University of California - Los Angeles, Los Angeles (2000).

Loewenhardt, P.K., Blackwell, B.D., Boswell, R.W., Conway, G.D., and Hamberger, S.M., Plasma production in a toroidal heliac by helicon waves, Phys. Rev. Lett., 67, 2792 (1991).

Mieno, T., Kamo, T., Hayashi, D., Shoji, T., and Kadota, K., Efficient production of $\mathrm{O}^{+}$ and $\mathrm{O}^{-}$ions in a helicon wave oxygen discharge, Appl. Phys. Lett., 69, 617 (1996).

Miljak, D.G., and Chen, F.F., Density limit in helicon discharges, Plasma Sources Sci. Technol., 7, 537 (1998).

Mouzouris, Y., and Scharer, J.E., Wave propagation and absorption simulations for helicon sources, Phys. Plasmas, 5, 4253 (1998).

Nakamura, K., Suzuki, K., and Sugai, H., Helicon wave measurements in an inductively coupled magnetoplasma, Aus. J. Phys., 48, 461 (1995).

Okamoto, N., Kasahara, F., and Ikoma, H., Growth of "oxide-less" GaN layer by heliconwave excited N/sub 2/-Ar plasma treatment of Al/GaAs structure, Japanese Journal of Applied Physics, Part 2 (Letters), 38, L424 (1999).

Perry, A.J., Vender, D., and Boswell, R.W., The application of the helicon source to plasma processing, Journal of Vacuum Science \& Technology B (Microelectronics Processing and Phenomena), 9, 310 (1991).

Sakawa, Y., Koshikawa, N., and Shoji, T., A higher-order radial mode of $\mathrm{m}=0$ helicon waves, Plasma Sources Sci. Technol., 6, 96 (1997).

Schlüter, H., Shivarova, A., and Tarnev, K., Effects of plasma-density inhomogeneity and collisions and their relation to maintenance of waveguide discharges by Trivelpiece-Gould modes: II. Numerical analysis, Plasma Sources Sci. Technol., 10, 267 (2001).

Scime, E.E., Boivin, R.F., Kline, J.L., and Balkey, M.M., Microwave interferometer for steady-state plasmas, Rev. Sci. Instrum., 72, 1672 (2001).

Scime, E.E., Keiter, P.A., Balkey, M.M., Bolvin, R.F., Kline, J.L., Blackburn, M., and Gary, S.P., Ion temperature anisotropy limitation in high beta plasmas, Phys.

Plasmas, 7, 2157 (2000). 
Scime, E.E., Keiter, P.A., Zintl, M.W., Balkey, M.M., Kline, J.L., and Koepke, M.E., Control of ion temperature anisotropy in a helicon plasma, Plasma Sources Sci. Technol., 7, 186 (1998).

Shamrai, K.P., Stable modes and abrupt density jumps in a helicon plasma source, Plasma Sources Sci. Technol., 7, 499 (1998).

Shamrai, K.P., and Taranov, V.B., Resonances and anti-resonances of a plasma column in a helicon plasma source, Phys. Lett. A, 204, 139 (1995).

Shamrai, K.P., and Taranov, V.B., Volume and surface RF power absorption in a helicon plasma source, Plasma Sources Sci. Technol., 5, 474 (1996).

Shats, M.G., Rudakov, D.L., Boswell, R.W., and Borg, G.G., Ion temperature and plasma flows in improved confinement mode in the H-1 heliac, Phys. Plasmas, 4, 3629 (1997).

Shinohara, S., Miyauchi, Y., and Kawai, Y., Dynamic plasma behaviour excited by $m= \pm 1$ helicon wave, Plasma Phys. Controlled Fusion, 37, 1015 (1995).

Shinohara, S., and Soejima, T., Trials of RF plasma production using different antenna geometries with magnetic field, Plasma Phys. Controlled Fusion, 40, 2081 (1998).

Shinohara, S., and Yonekura, K., Discharge modes and wave structures using loop antennae in a helicon plasma source, Plasma Phys. Controlled Fusion, 42, 41 (2000).

Shivarova, A., and Tarnev, K., Effects of plasma-density inhomogeneity and collisions and their relation to maintenance of waveguide discharges by Trivelpiece-Gould modes: I. Resonance absorption, Plasma Sources Sci. Technol., 10, 260 (2001).

Shoji, T., Sakawa, Y., Nakazawa, S., Kadota, K., and Sato, T., Plasma production by helicon waves, Plasma Sources Sci. Technol., 2, 5 (1993).

Stix, T.H., Radiation and Absorption Via Mode Conversion in an Inhomogeneous Collision Free Plasma, Phys. Rev. Lett., 15, 878 (1965).

Takeno, H., Yasaka, Y., Sakai, O., and Itatani, R., High density central cell plasma production and ion heating by helicon wave damping and its mode conversion into a slow Alfven wave, Nucl. Fusion, 35, 75 (1995).

Trivelpiece, A.W., and Gould, R.W., J. Appl. Phys., 11, 1784 (1959).

Wong, K.L., and Ono, M., Parametric decay of lower-hybrid waves in the ACT-1 toroidal device, Phys. Rev. Lett., 47, 842 (1981).

Yun, S., Kim, J.H., and Chang, H.Y., Frequency dependence of helicon wave plasmas near lower hybrid resonance frequency, J. Vac. Sci. Technol. A, 15, 673 (1997).

Yun, S., Suwon, C., Tynan, G., and Hongyoung, C., Density enhancement near lower hybrid resonance layer in $m=0$ helicon wave plasmas, Phys. Plasmas, 8, 358 (2001).

Yun, S.M., and Chang, H.Y., Radial density profile change near lower hybrid frequency in $m=0$ helicon wave plasmas, Phys. Lett. A, 248, 400 (1998). 


\section{Chapter 5: Ion Temperature Measurements}

Since the first ion temperature measurements in HELIX, anomalous ion heating in helicon sources has been a topic of study at WVU. In 2000, Matthew Balkey completed a comprehensive study of ion heating as a function of RF driving frequency and magnetic field for five different RF antennas and an all Pyrex vacuum chamber [Balkey, 2000]. Those measurements demonstrated a correlation between ion heating, plasma density production, and the lower hybrid frequency. As discussed in Chapter 4, wave numbers for the slow wave peak for driving frequencies just below the lower hybrid frequency. The predicted wave number magnitudes are sufficiently large (the slow wave phase velocities are small enough) for ion Landau damping to be responsible for the observed ion heating. If ion Landau damping of slow waves is responsible for the ion heating, the ions should be heated preferentially in the perpendicular direction and the ion heating should peak near the plasma edge because the slow wave is heavily damped as it propagates from the plasma edge towards the center. Balkey's experiments did show that the ions were preferentially heated in the perpendicular direction, but could not address the issue of edge ion heating because they were performed at a single axial location and at the center of the source [Balkey et al., 2001]. In this Chapter, ion temperature measurements at multiple axial and radial locations are presented and discussed for the two different magnetic field geometries discussed in Section 2.3: the ten electromagnets configuration (Section 5.1) and the eight electromagnets configuration (Section 5.2). The ion temperature observations are discussed in terms of the expected signatures for the slow wave in Section 5.3.

\subsection{The Ten Electromagnet Configuration}

In the ten electromagnets configuration, all ten of the HELIX electromagnet coils were connected in series. With all ten electromagnets energized, the maximum magnetic field strength in HELIX was 1125 Gauss. Operating the source at RF frequencies lower than $8.5 \mathrm{MHz}$ was problematic in the ten coil configuration due an inability to obtain a good antenna match, i.e., the capacitance in the matching circuit was insufficient to compensate for the inductance of the antenna and transmission line. The ten coil 
configuration did have the advantage that the magnetic field lines at the edge of the plasma did not terminate on the conducting vacuum chamber wall in HELIX. The unencumbered magnetic field geometry in the ten coil configuration is more appropriate for comparison to idealized helicon source models.
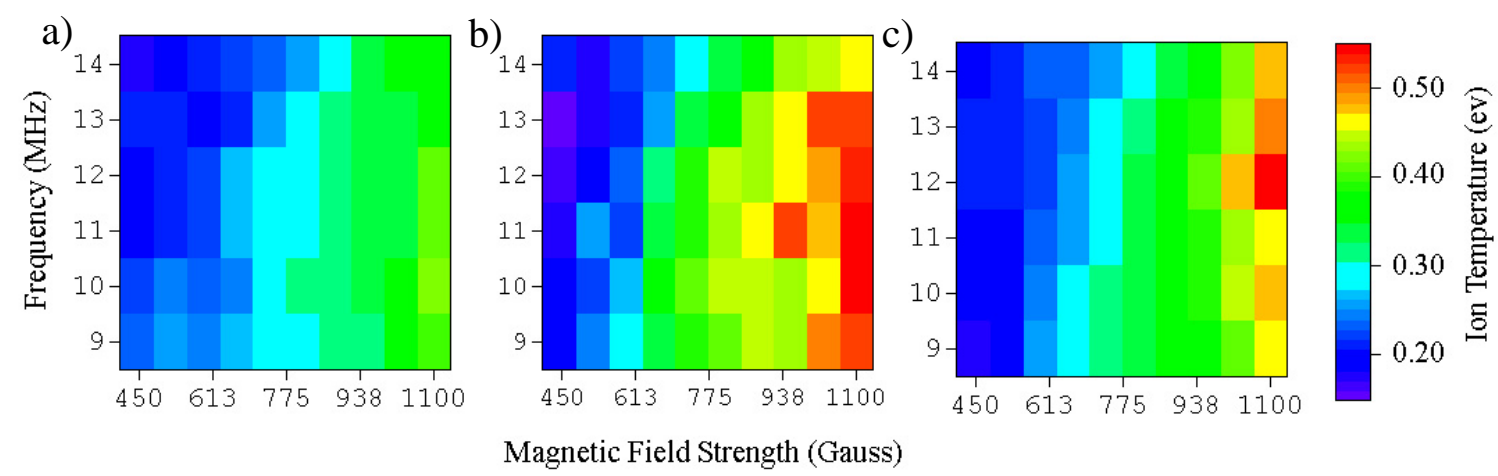

Figure 59: The perpendicular ion temperature verses driving (RF) frequency and magnetic field strength. a) $5 \mathrm{~cm}$ from antenna. b) $35 \mathrm{~cm}$ from antenna. c) $65 \mathrm{~cm}$ from antenna.

The perpendicular ion temperature in the ten coil configuration was measured at three different axial locations. Figure 59a, Figure 59b, and Figure 59c show the perpendicular ion temperature as a function of magnetic field strength and driving frequency for axial locations of 5,35 , and $65 \mathrm{~cm}$ from the antenna, respectively. The maximum perpendicular ion temperatures occur at the $35 \mathrm{~cm}$ location, suggesting that the ion heating has a maximum downstream from the antenna. A peak in the ion heating downstream from the antenna is reminiscent of measurements of a peak in the plasma density downstream from the antenna [Chen, 1996; Sudit and Chen, 1996; Chen and Boswell, 1997]. The peak in the perpendicular ion temperature downstream from the antenna indicates that the parallel ion thermal conductivity in helicon sources is small compared to the radial diffusion time of the ions; most likely due to the high ion-ion and ion-neutral collision frequency in the source.

At the lowest magnetic field strengths $(\sim 450 \mathrm{G})$, the perpendicular ion temperatures at the $5 \mathrm{~cm}$ location are slightly higher than the $35 \mathrm{~cm}$ location (Figure 59b) and the 65 $\mathrm{cm}$ location. Contrary to what is observed at the higher magnetic field strengths, the ion temperatures monotonically decrease with increasing distance from the antenna. Figure $60 \mathrm{a}$ shows a plot of the ion temperature at the $5 \mathrm{~cm}$ location subtracted from the ion 
temperatures at the $35 \mathrm{~cm}$ locations, $\Delta T_{i}=T_{i}(z=35 \mathrm{~cm})-T_{i}(z=5 \mathrm{~cm})$, as a function of RF driving frequency and magnetic field strength. Negative values indicate regions where the ion temperatures at the $5 \mathrm{~cm}$ location are greater than the $35 \mathrm{~cm}$ location. Figure $60 \mathrm{~b}$ is the same data as Figure $60 \mathrm{a}$, but for a single frequency. At magnetic fields below 550 Gauss, the ion temperatures monotonically decrease with distance from the antenna, but as the magnetic field increases, the ion temperatures peak at a finite distance from the antenna. Another feature shown in Figure $60 \mathrm{~b}$ is that the rate at which the ion temperatures increase at the $35 \mathrm{~cm}$ location compared with the $5 \mathrm{~cm}$ location as a function of magnetic field strength. Below 750 Gauss the ion temperatures at the $35 \mathrm{~cm}$ location increase at a constant rate compared to the $5 \mathrm{~cm}$ location. Above 750 Gauss, the ion temperatures both increase at the same rate. The dependence of the axial ion temperature profile (axial electron density profile) on magnetic field strength observed in these experiments (elsewhere [Boswell et al., 1989; Sudit and Chen, 1996; Chen et al., 2000]) illustrate the dramatic differences in helicon source characteristics for slightly different operational parameters.

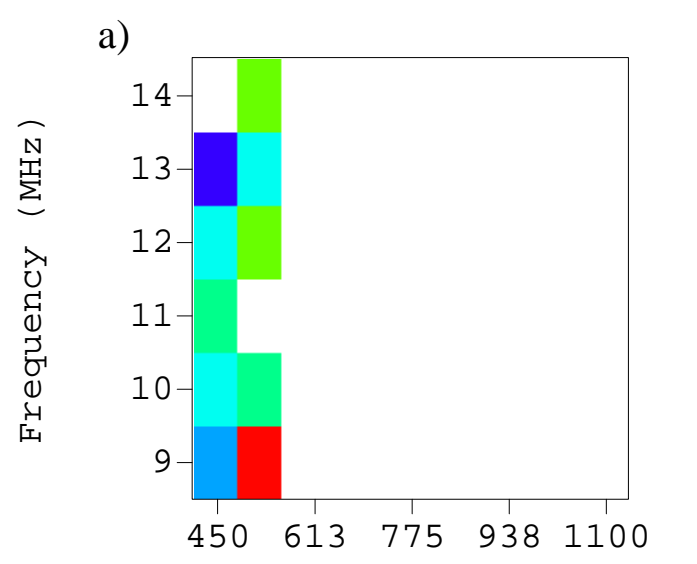

Magnetic Field Strength (Gauss)

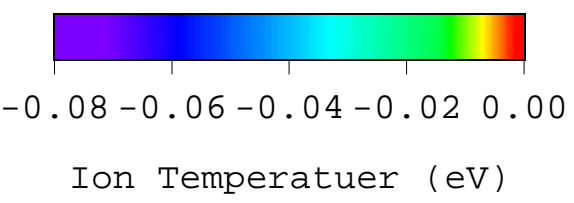

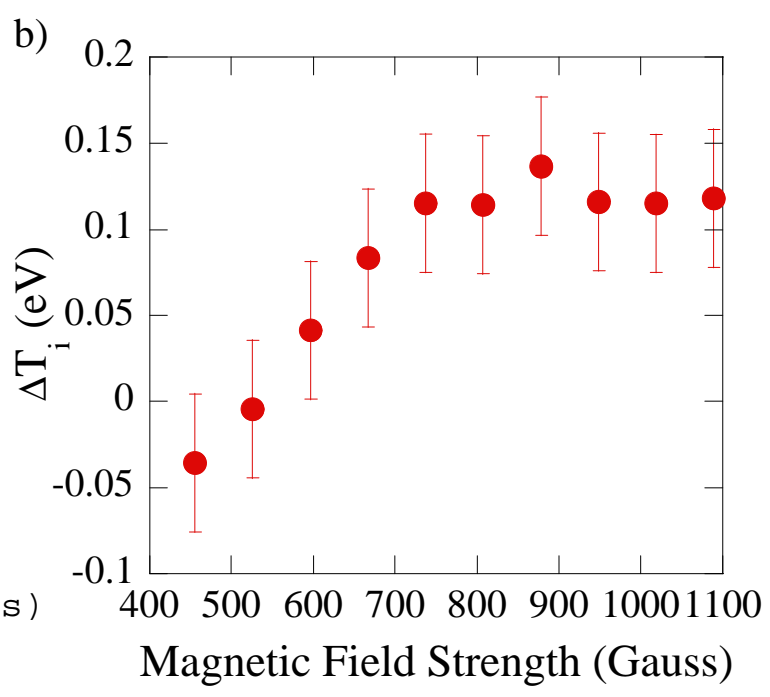

Magnetic Field Strength (Gauss)

Figure 60: a) The difference in the perpendicular ion temperature at the antenna $(\mathrm{z}=5 \mathrm{~cm})$ and downstream $(\mathrm{z}=35)$ locations as a function of RF driving frequency and magnetic field strength limited to $\Delta T_{i}<0.8 \mathrm{eV}$ and b) at $9 \mathrm{MHz}$ as a function of magnetic field strength. 
The perpendicular ion temperatures presented in Figure 59 do not indicate a strong dependence on driving frequency. For frequencies of $13 \mathrm{MHz}$ and less, the ion temperatures all increase with magnetic field strength in approximately the same manner. The perpendicular ion temperatures observed in these experiments are about half the maximum values reported by Balkey et al. [2001].

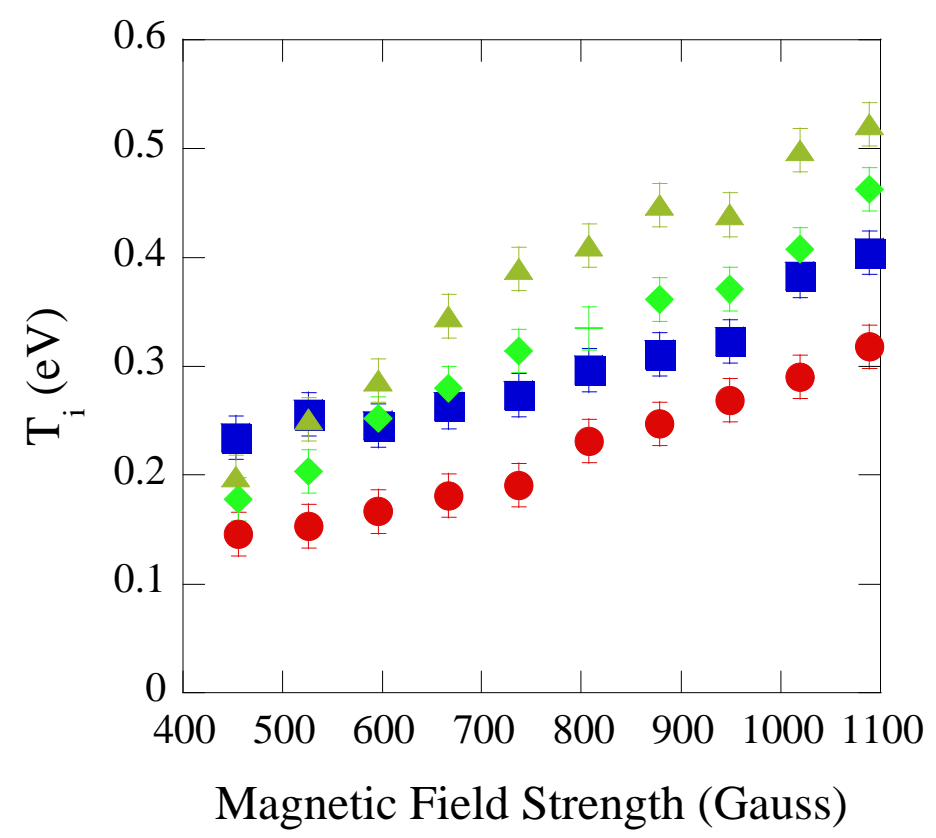

Figure 61: The perpendicular ion temperature versus magnetic field strength for four different axial

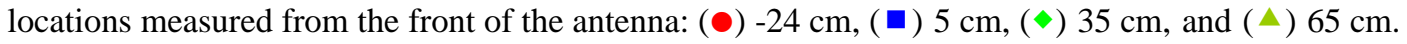

A detailed examination of the perpendicular ion temperature suggests that the mechanism responsible for ion heating near the antenna is distinctly different than the downstream ion heating mechanism. Figure 61 shows the perpendicular ion temperature as function of magnetic field strength for a RF frequency of $9 \mathrm{MHz}$ at four different axial locations. Again, at low magnetic field strengths, the perpendicular ion temperature near the antenna is higher than at the other locations. The peak in the downstream perpendicular ion temperature appears as the magnetic field strength increases. Note that the slope of the perpendicular ion temperature verses magnetic field strength curve near the antenna is significantly different than at the other locations, suggesting that the ion heating mechanism responsible near the antenna is different than the ion heating mechanism at the other downstream locations. Also shown in Figure 61 is the perpendicular ion temperature on the backside of the antenna $(-24 \mathrm{~cm}$ from the front of 
the antenna). The perpendicular ion temperature on the backside of the antenna also increases with increasing magnetic field strength, but is lower than for all three of the downstream locations. The difference in the ion temperatures in the front and the back of the antenna clearly indicate directional coupling of the RF energy into the ions.

Using the two dimensional LIF system at the $65 \mathrm{~cm}$ axial location, the perpendicular ion temperature, the parallel ion temperature, and the metastable ion density were measured as a function of radial position. Radial profiles of the perpendicular ion temperature are shown in Figure 62a for four different magnetic field strengths. For the three lowest magnetic field strengths, there is a statistically significant trend in the perpendicular ion temperature radial profiles. The perpendicular ion temperatures peak on axis and at the edge. The parallel ion temperature at the same four magnetic field strengths is shown in Figure 62b. At the lowest magnetic field strength, the parallel ion temperature decreases from about $1.5 \mathrm{~cm}$ to $3 \mathrm{~cm}$. At higher magnetic field strengths, the parallel ion temperatures are slightly peaked at the edge. Comparing the perpendicular and parallel ion temperatures at each of the four magnetic field strengths, the ion temperature anisotropy $\left(T_{\perp} / T_{\|}\right)$remains about the same for all magnetic field strengths, $T_{\perp} / T_{\|} \sim 2.6$.
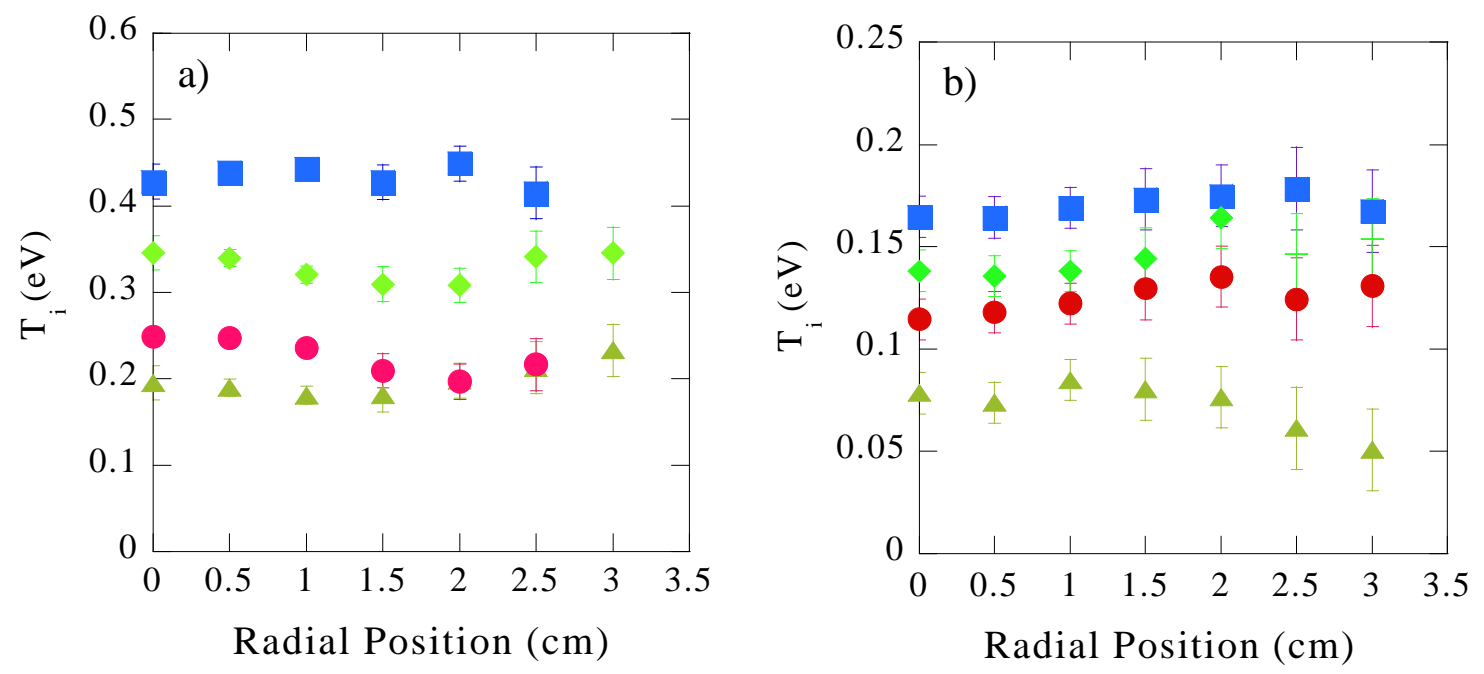

Figure 62: Radial variation of perpendicular (a) and parallel (b) ion temperature for four different magnetic field strengths: $(\bullet) 515$ Gauss, (•) 656 Gauss, $(\diamond) 868$ Gauss, and (•) 1080 Gauss 
In addition to the ion temperature profiles, metastable ion density profiles were also calculated from the two-dimensional LIF system data at $z=65 \mathrm{~cm}$. The metastable ion density is proportional to the integral over laser frequency of the measured LIF signal. The measured metastable ion density profiles are relative plasma density measurements assuming that the electron temperature is constant across the radial range of the measurement and that electron impact excitation of ions is the dominant production mechanism for creation of the metastable ion state pumped by LIF. Details of the relationship between LIF signal and metastable density were described in Chapter 1 .

The measured metastable density profiles for four magnetic field strengths are shown in Figure 63. Each profile was normalized to the peak value to account for changes in the bulk plasma parameters, e.g., plasma density, ion temperature, and electron temperature. All of the metastable ion density profiles are peaked. In contrast to reports from other groups operating helicon sources near the lower hybrid frequency [Yun and Chang, 1998; Sakawa et al., 1999; Yun et al., 2001], there is no evidence of hollow ion density profiles. The LIF measured peaked density profiles at these source parameters are consistent with in-situ Langmuir probe measurements. Quantitative comparison between the slopes of the LIF and Langmuir probe density profiles is problematic, as the metastable density profiles should decrease with radius more quickly than the plasma density due to quenching of the metastable ion state through neutral collisions as the ArI population increases with radius [Skiff et al., 2001]. Also evident in Figure 63 is a shift in the center of the ion metastable density profile at the highest magnetic field strength. Although not observed for all high magnetic field strength operational schemes, the outward shift of the peak plasma density is regularly observed at high magnetic field strengths. The cause of the outward shift in the profile is not yet known. Asymmetries in the RF antenna system or magnetic field errors that only have an effect at high magnetic field strengths are two explanations under consideration 


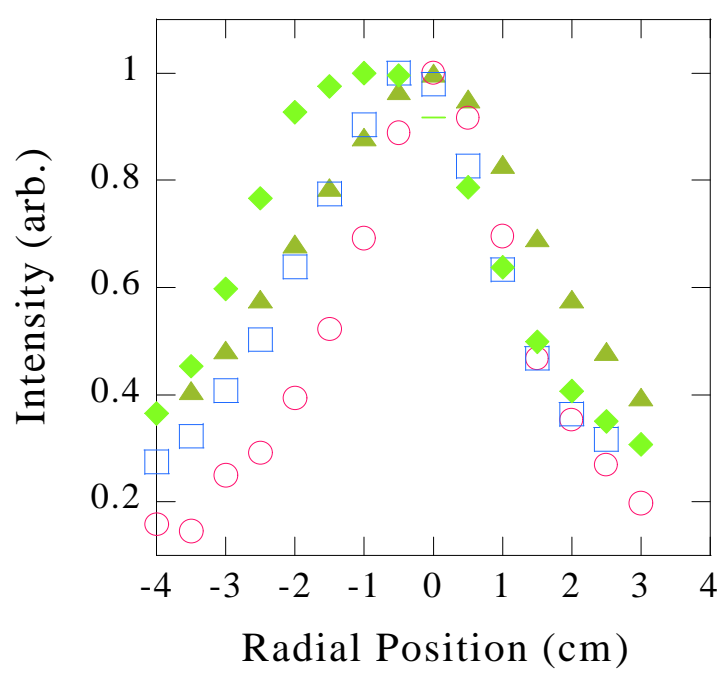

Figure 63: Metastable ion density profiles at $z=65 \mathrm{~cm}$ for four different magnetic field strengths: $(\triangle) 515$ Gauss, (०) 656 Gauss, (») 868 Gauss, and ( $\square$ ) 1080 Gauss.

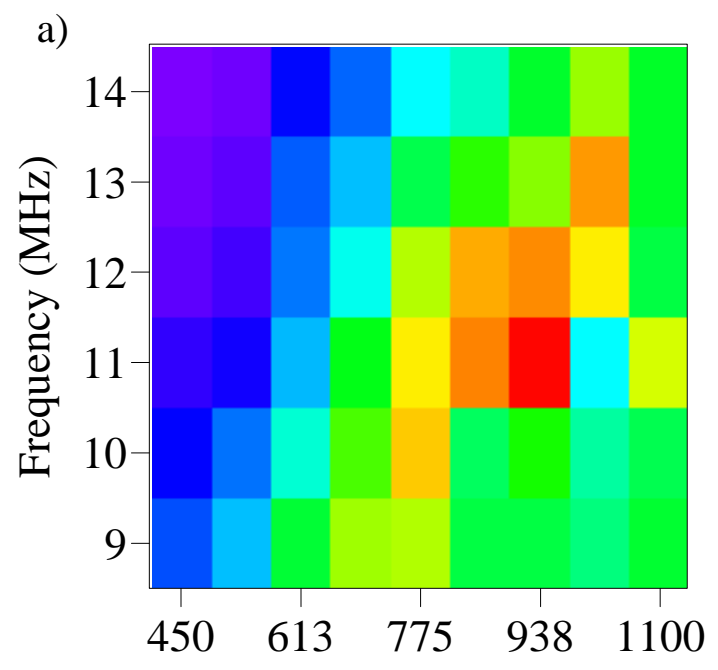

Magnetic Field Strength (Gauss)

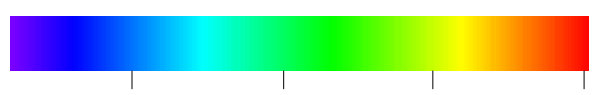

b)

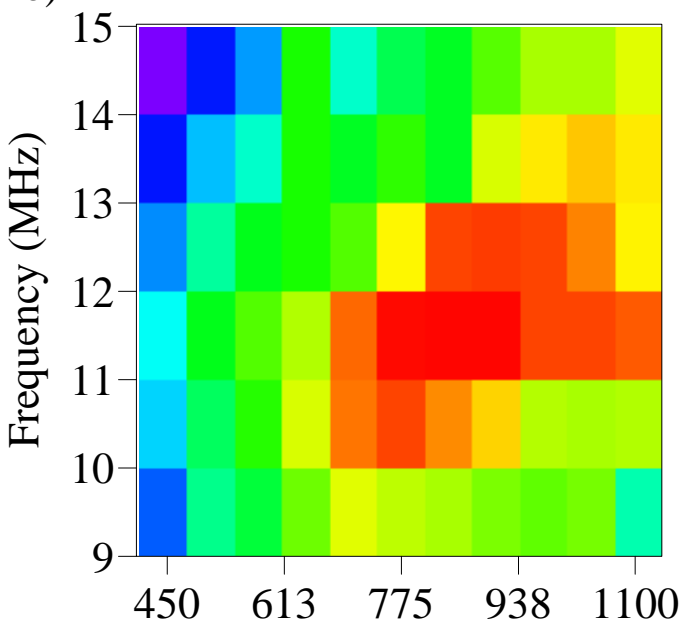

Magnetic Field Strength (Gauss)

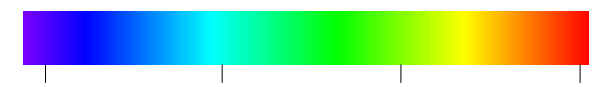

0.05

0.15

0.20

Density $\left(10^{12} \mathrm{~cm}^{-3}\right)$

Density $\left(10^{12} \mathrm{~cm}^{-3}\right)$

Figure 64: Electron density verses RF frequency and HELIX magnetic field strength in LEIA for (a) these experiments and (b) the Balkey et al. [Balkey et al., 2001] experiments.

Another key feature of the ten electromagnet coil configuration is the dependence of the electron density on RF frequency and magnetic field strength. Electron density measurements in LEIA (shown in Figure 64a) are consistent both quantitatively and 
qualitatively with those of Balkey et al. [2001] (Figure 64b). The perpendicular ion temperatures reported in Balkey et al. [2001], however, differ considerably from the data shown in Figure 59. In Balkey's experiment, the perpendicular ion temperature (measured approximately $55 \mathrm{~cm}$ downstream from the edge of the RF antenna) peaked only at the highest magnetic field strengths $(1200 \mathrm{G})$ and the lowest RF frequencies $(\sim 8.5$ $\mathrm{MHz}$ ). The similarity in plasma density measurements suggests that the density production mechanisms in completely glass (Pyrex) and hybrid (part glass and part conductor) vacuum chambers are the same. In both types of vacuum chambers, the electron density in LEIA is enhanced when the RF driving frequency equals the on-axis, HELIX lower hybrid frequency. The difference in ion heating characteristics suggests that boundary conditions play an important role in ion heating in helicon sources.

\subsection{The Eight Electromagnet Configuration}

With the last two electromagnets before the LEIA chamber removed, higher currents could be realized in the HELIX electromagnets without reaching the voltage limit of the available power supply (see Section 2.3). In the eight coil configuration, the maximum magnetic field strength in HELIX increased to $1320 \mathrm{G}$. Apart from being able to match the antenna at frequencies as low as $7.5 \mathrm{MHz}$, the main difference between the eight and ten coil configurations is that in the eight coil configuration, the magnetic field lines near the edge of the plasma in HELIX intersect the conducting vacuum chamber wall just before the LEIA chamber while the inner magnetic field lines do not. This complex magnetic field geometry is not easily described with a single, simple theoretical model. The intersection of the magnetic field lines with the conducting chamber wall imposes a conducting axial boundary condition on the edge region of the plasma, thereby making the physical geometry of the edge of the plasma similar to the model used by Cho [2000] in his study of lower hybrid resonance effects in helicon sources. The intersection of the edge magnetic field lines with a conductive boundary also occurred in the Balkey et al. [2001] experiments. In those experiments, a stainless steel bellows connected the Pyrex chamber to LEIA and the divergence of the magnetic field at the HELIX-LEIA interface resulted in the edge magnetic field lines intersecting the conductive bellows. It is important to note that at all LIF measurement locations, the deviation of the magnetic 
field direction from the axial direction is too small to cause a significant mixing of perpendicular and parallel ion velocity components.

Perpendicular and parallel ion temperatures were measured at two different axial locations in the eight coil configuration (Figure 65). As in the ten coil configuration, the perpendicular and parallel ion temperatures downstream from the antenna $(z=35 \mathrm{~cm})$ are higher than in front of the antenna $(z=5 \mathrm{~cm})$. At both locations, the ions are anisotropic; the perpendicular ion temperatures are higher than the parallel ion temperatures. At the $35 \mathrm{~cm}$ location, the maximum perpendicular ion temperature is twice the maximum ion temperature observed in the ten coil configuration and comparable to those observed in the all Pyrex HELIX experiments Balkey et al., [2001]. The ion temperature measurements as a function of RF driving frequency and magnetic field strength at both locations clearly indicate a connection between ion heating and the lower hybrid frequency (Figure 65). For RF frequencies below the on-axis lower hybrid frequency (shown by the solid white line in Figure 7), the perpendicular and parallel temperatures at both locations are higher than for RF frequencies above the lower hybrid frequency. Although the shapes of the enhanced ion temperature regions are roughly similar at both locations, there are some significant differences between the ion heating near the antenna and farther downstream. In the downstream $(z=35 \mathrm{~cm})$ perpendicular ion temperature data (Figure 65a), there is a well-defined peak, $T_{i} \sim 1 \mathrm{eV}$, in the ion temperature around an Rf frequency of $9 \mathrm{MHz}$ and the highest magnetic field strengths. Near the antenna $(z=5 \mathrm{~cm})$, there is no such peak and for the lowest RF frequencies, less than $8.5 \mathrm{MHz}$, the perpendicular ion temperature does not increase for RF frequencies equal to the onaxis lower hybrid frequency (Figure 65c). For low RF frequencies, the perpendicular ion temperature near the antenna and the parallel ion temperature downstream (Figure 65b) increase only at the highest magnetic field strengths. Note also that near the antenna, increases in the parallel ion temperature appear completely unrelated to the lower hybrid resonance condition. 
Perpendicular
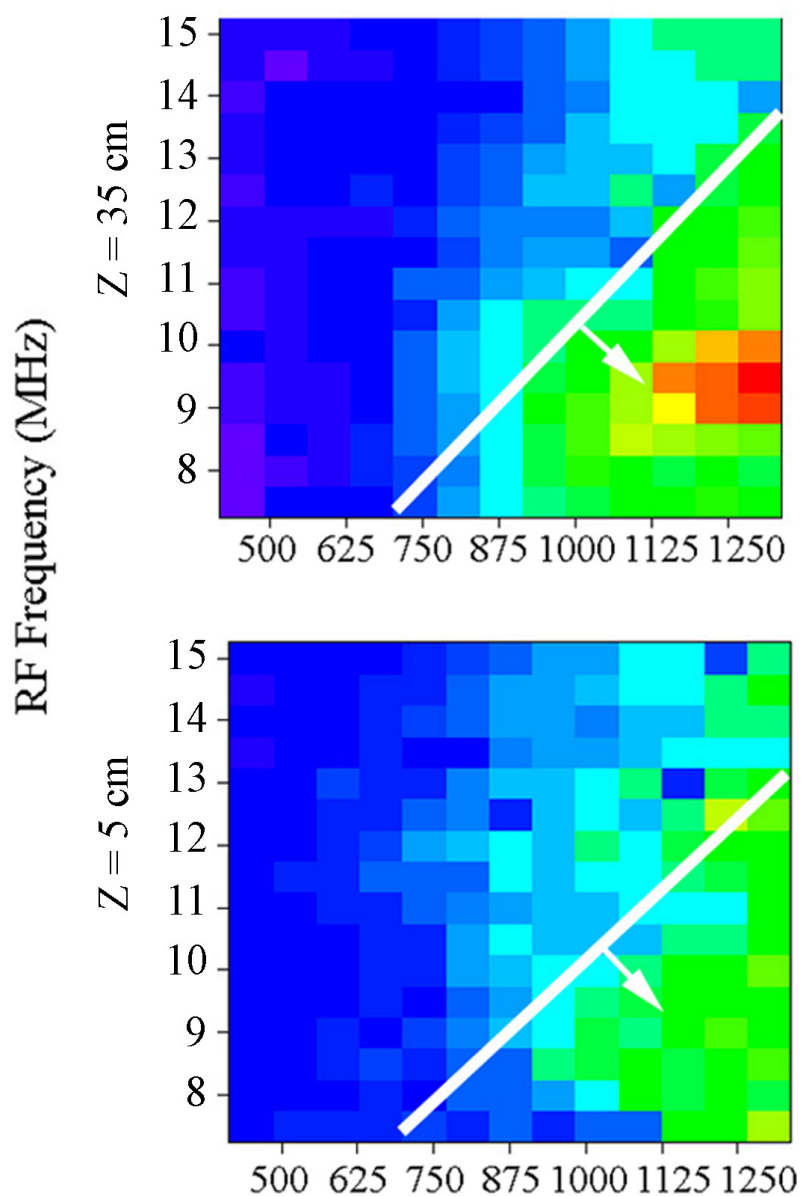

Parallel
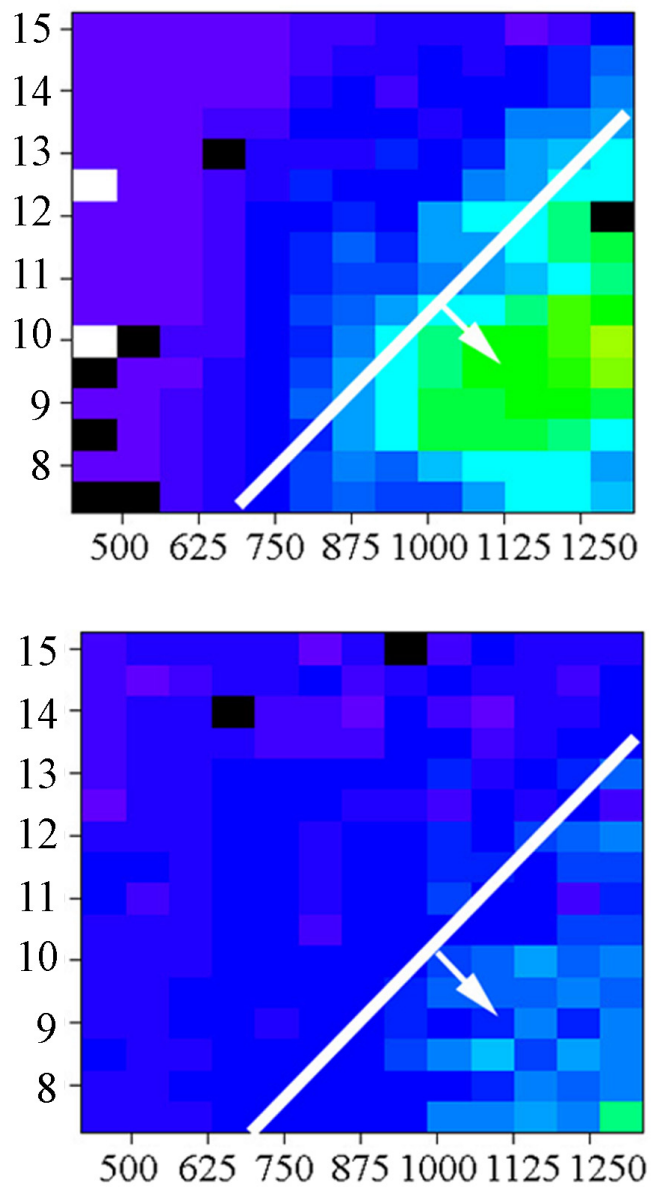

Magnetic Field Strength (Gauss)

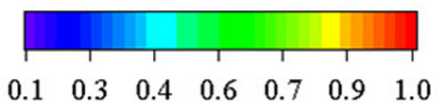

\section{Ion Temperature (eV)}

Figure 65: Ion temperature verses frequency and magnetic field in the perpendicular and parallel direction for $z=35 \mathrm{~cm}$ location and $z=5 \mathrm{~cm}$ location. The white line is where the RF driving frequency is equal to the on axis lower hybrid frequency and the arrow points in the direction the line would move for lower plasma densities, i.e. at the edge of the plasma.

According to calculations of slow wave propagation, the TG mode, the slow waves excited at the edge of helicon sources are heavily damped as they propagate towards the center of the plasma. Therefore, if slow waves play a role in coupling RF energy into the ions, the ion heating should occur near the plasma edge. Perpendicular and parallel ion temperature profile measurements at $z=35 \mathrm{~cm}$ for three different sets of RF frequencies and magnetic field strengths are shown in Figure 66, Figure 67, and Figure 68. Each set 
of RF frequency and magnetic field strength values corresponds to a different ion temperature region in Figure 65.

At a high RF frequency (13 MHz) and low magnetic field strength (571 G), Figure 66 is representative of the low ion temperatures observed when the RF frequency is well above the on-axis lower hybrid frequency. The perpendicular ion temperatures appear independent of radius. There is a small decrease in parallel ion temperatures with increasing radius; from $0.11 \mathrm{eV}$ on axis to $0.08 \mathrm{eV}$ towards the edge of the plasma. The parallel ion temperatures are four to five times room temperature and could result from either thermalization of the perpendicular ion temperature by ion-ion collisions or ion heating resulting from electron-ion collisions [Balkey et al., 2001]. The perpendicular ion temperatures are well above room temperature and cannot be explained by electronion thermal collisional equilibration [Balkey et al., 2001].

At an RF frequency of $10.5 \mathrm{MHz}$ and a magnetic field strength of $878 \mathrm{G}$, Figure 67 is representative of ion heating at RF frequencies near the lower hybrid frequency. As a function of radius, the perpendicular ion temperatures are constant to within experimental error. The parallel ion temperatures decrease slightly with increasing radius.

At an RF frequency of $9 \mathrm{MHz}$ and a magnetic field strength of $1185 \mathrm{G}$, Figure 68 corresponds to the hot perpendicular ion temperature region seen in Figure 65a. In this case, the perpendicular ion temperatures are constant in the center of the source and increase sharply at the edge of the plasma. The increase in perpendicular ion temperature at the edge of the source is larger than the experimental error in the measurement and is reproducible. The parallel ion temperatures are peaked on axis and decrease by fifty percent towards the edge of the plasma. That the largest parallel ion temperatures occur where the plasma density is the greatest suggests that thermal equilibration of the ions (due to ion-ion collisions) is responsible for heating the ions in the parallel direction. Towards the edge, the plasma density drops and ion confinement is poorer. Thus, the parallel ion temperature decreases towards the edge. 


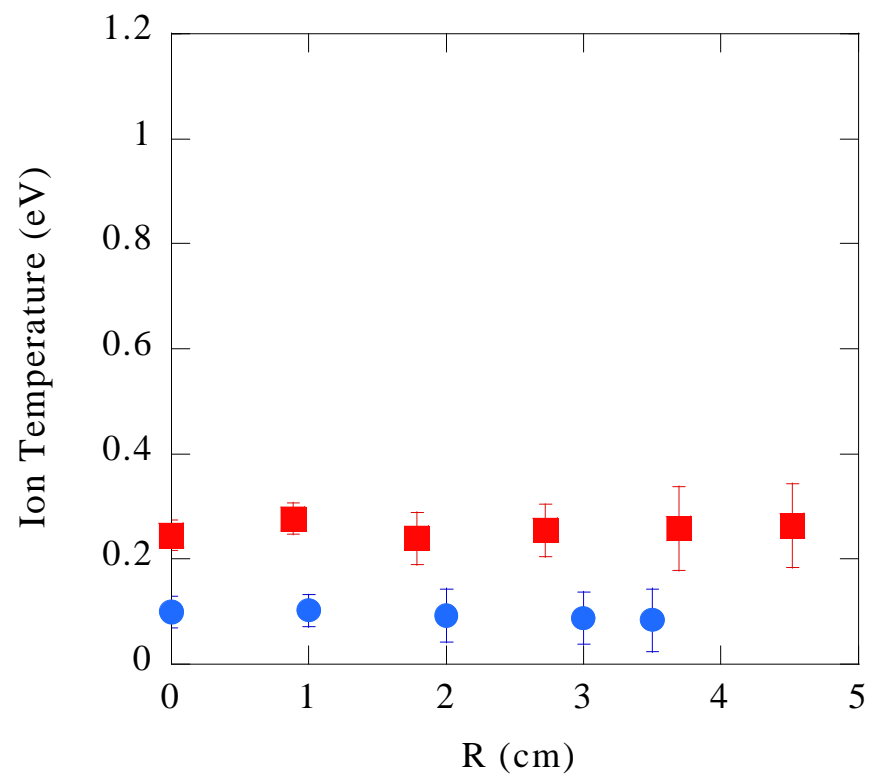

Figure 66: ( ) Perpendicular and (๑) parallel ion temperature verses radius for magnetic field $=571$ Gauss, pressure $=6.7 \mathrm{mTorr}$, RF frequency $=13 \mathrm{MHz}$, RF power $=750 \mathrm{~W}$.

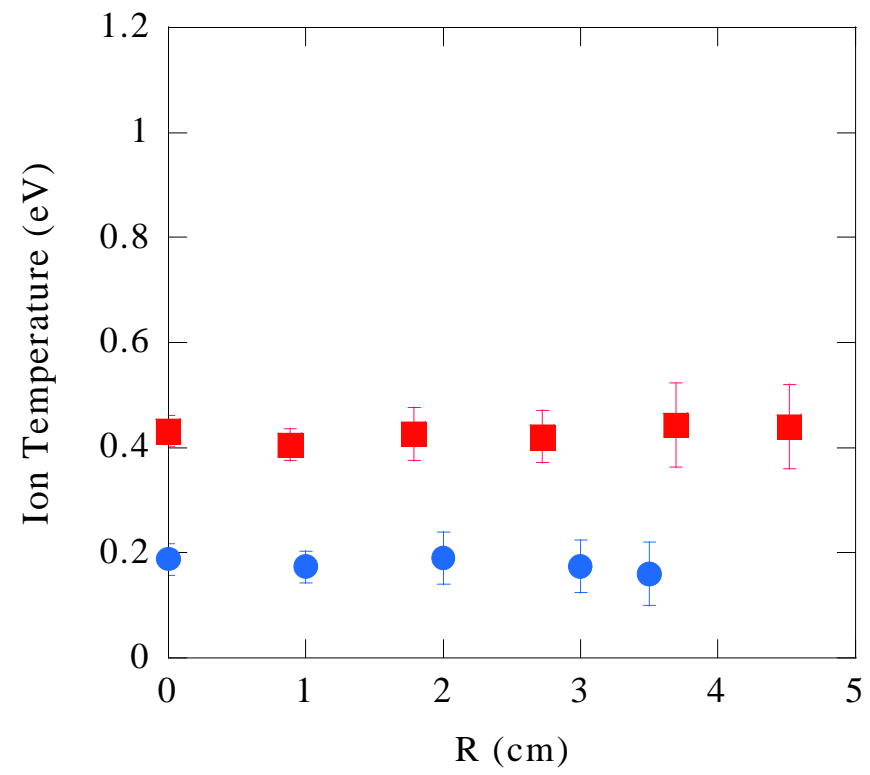

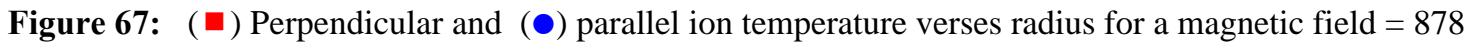
Gauss, pressure $=6.7 \mathrm{mTorr}, \mathrm{RF}$ frequency $=10.5 \mathrm{MHz}, \mathrm{RF}$ power $=750 \mathrm{~W}$ 


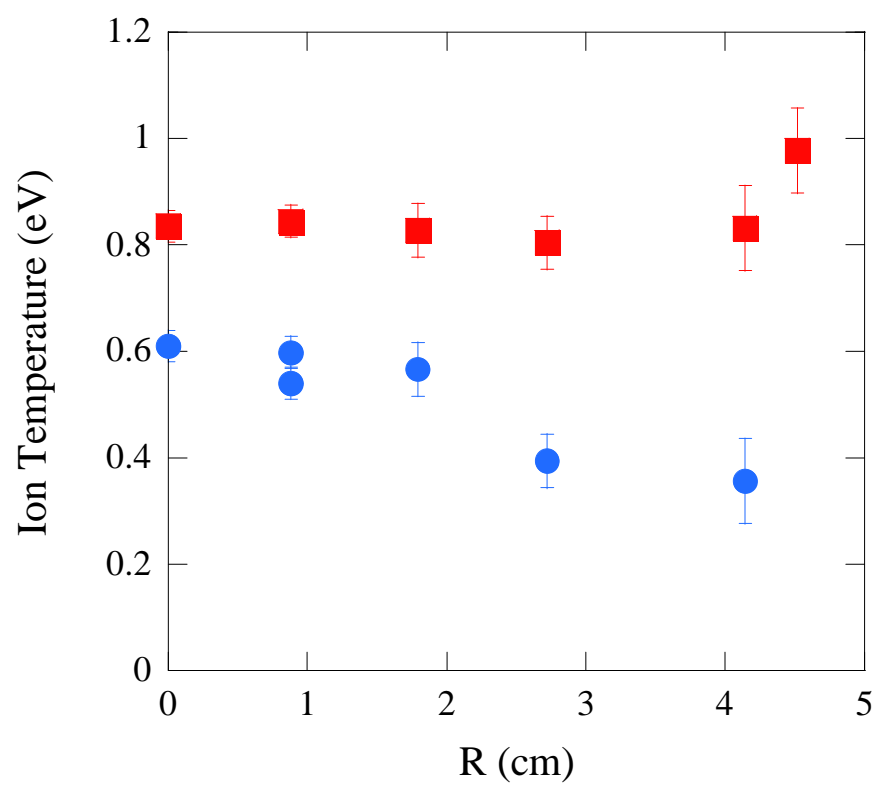

Figure 68: ( $\square)$ Perpendicular and $(\bullet)$ parallel ion temperature verses radius for magnetic field $=1185$ Gauss, pressure $=6.7 \mathrm{mTorr}, \mathrm{RF}$ frequency $=9 \mathrm{MHz}, \mathrm{RF}$ power $=750 \mathrm{~W}$

\subsection{Implications of Ion Temperature Measurements}

The objective of the ion heating measurements presented in this work was to identify the mechanism or mechanisms responsible for ion heating in helicon sources. The experimental results suggest two possible anomalous (not collisional equilibration) mechanisms: coupling of RF energy into the ions through damping of the slow wave and ion heating due to damping of parametrically driven instabilities. A discussion of the possible role in ion heating by parametrically driven instabilities will be presented in Chapter 7. In this section, the discussion will focus on how the ion temperature data support the conclusion that the ions are heating through ion Landau damping of the slow wave at the edge of plasma.

In a collisionless plasma, the slow electromagnetic wave experiences a resonance at the lower hybrid frequency [Cho, 2000]. At the resonance, the perpendicular wave number of the wave increases to infinity and the slow wave becomes electrostatic. Although collisions prevent the perpendicular wave number from becoming infinite at the resonance, for typical helicon source parameters the perpendicular wave number is still very large at the lower hybrid resonance [Cho, 2000]. At large perpendicular wave 
numbers, the perpendicular phase speed of the slow wave, $\omega / k_{\perp}$, can be comparable to the ion thermal speed. Under these circumstances, the ions can Landau damp the slow wave, thereby gaining energy [Swanson, 1989]. Since the ion Landau damping occurs along the perpendicular direction, the ions will be preferentially heated in the perpendicular direction. The simple slow wave model presented in Chapter 4 confirmed that the perpendicular wave number of the slow wave in HELIX can become large enough for ion Landau damping to occur. Because density profile effects shift the lower hybrid resonance towards the plasma edge (see Chapter 4), ion heating due to Landau damping of the slow wave should occur near the edge of the plasma.

Ion temperature measurements in the eight coil configuration clearly indicate perpendicular ion heating at the plasma edge (Figure 68). The perpendicular ion temperatures can be constant in the center of the discharge only if the perpendicular ion heating occurs off axis or if the ion thermal conductivity along the magnetic field is large enough to balance on-axis ion heating. Otherwise, cross-field diffusion would create a peaked ion temperature profile. The strong gradient in parallel ion temperatures along the axis of the helicon source (Figure 61) argues against a high ion thermal conductivity along the magnetic field. Therefore, the flat center and peaked edge of the perpendicular ion temperature radial profile in the maximum heating case (Figure 68) are strong evidence of edge perpendicular ion heating in the helicon source.

Close investigation of the differences in the ion temperature along the magnetic field has also revealed additional evidence in support of the slow wave, ion heating hypothesis. Subtraction of the perpendicular ion temperature near the antenna from the measurements at the $z=35 \mathrm{~cm}$ location provides a measure of the additional ion heating occurring downstream of the antenna. The perpendicular ion temperature difference, $\Delta T_{i}=T_{i}(z=35 \mathrm{~cm})-T_{i}(z=5 \mathrm{~cm})$, in the ten coil configuration is shown in Figure 69 as a function of RF driving frequency and magnetic field strength. The ion temperature difference in the ten coil configuration is a maximum when the RF frequency is near or just below the on-axis lower hybrid frequency (Figure 69). Apart from the general correlation with lower hybrid frequency, there are no other distinct features in the temperature difference data. 


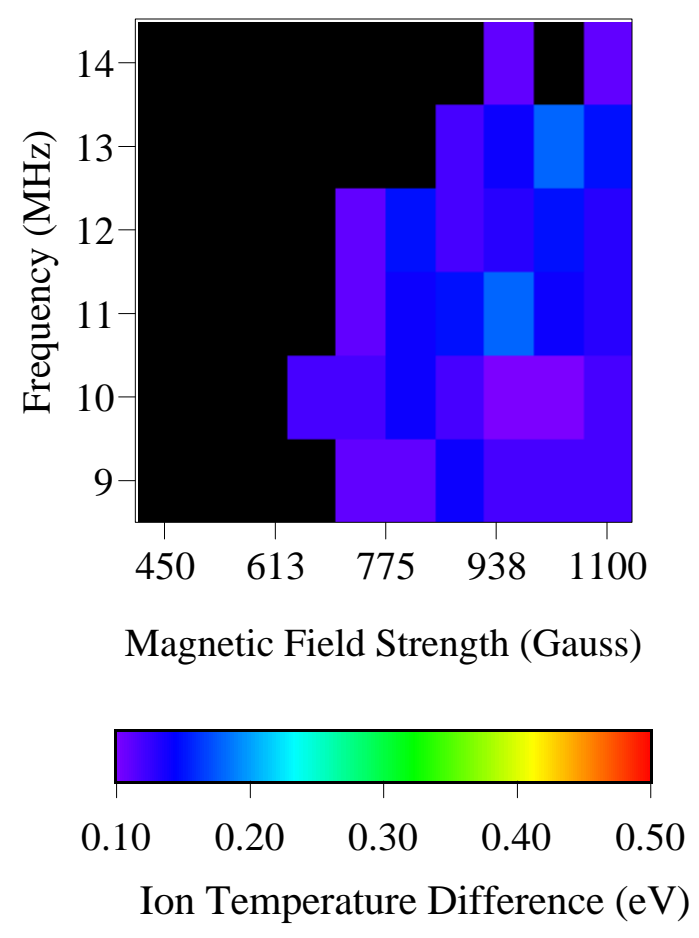

Figure 69: Difference in the perpendicular ion temperature at the $z=35 \mathrm{~cm}$ and $z=5 \mathrm{~cm}$ locations in the ten coil configuration.

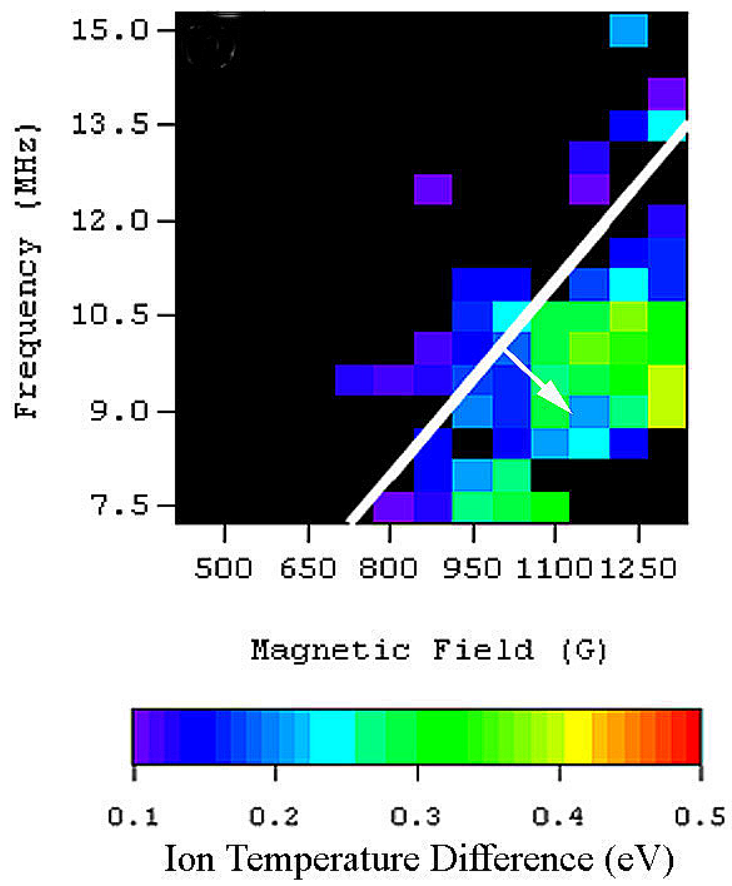

Figure 70: Difference in the perpendicular ion temperature at the $z=35 \mathrm{~cm}$ and $z=5 \mathrm{~cm}$ locations in the eight coil configuration. 
The perpendicular ion temperature data from the eight coil configuration, however, tell a remarkably different story. The perpendicular ion temperature difference in the eight coil configuration is shown in Figure 70 as a function of RF driving frequency and magnetic field strength. There is still a strong correlation between additional ion heating in the eight coil configuration and when the RF frequency is below the lower hybrid frequency (the white line in Figure 70 indicates where the RF driving frequency matches the on axis lower hybrid frequency respectively), but in the eight coil configuration a distinct peak in the downstream perpendicular ion temperature appears around an RF frequency of 9.5 MHz and a magnetic field strength of 1200 Gauss.

In Figure 71 , the normalized perpendicular wave numbers, $k_{\perp} v_{t h i} / \omega$, calculated with the simple model described in Chapter 4 are plotted verses RF frequency and magnetic field strength. For comparison, the perpendicular ion temperatures at the $35 \mathrm{~cm}$ location shown in Figure 65 are replotted in Figure 71b. Both the broad trends and the location of the peaks in Figure 71a and Figure $71 \mathrm{~b}$ are quantitatively consistent. Where the model predicts the largest perpendicular wave numbers, the downstream ions are most strongly heated in the perpendicular direction. The model predicts peak values for the normalized wave numbers on the order of 0.2, i.e. phase speeds about 5 times the ion thermal speed. Landau damping of waves with comparable phase to thermal speed ratios has been observed in other experiments [Wong and Ono, 1981].

Note that the shift in the ion heating peak towards RF frequencies and magnetic field strengths such that the RF frequency is well below the on-axis lower hybrid frequency is easily explained in terms of damping of slow waves without resorting to any modeling arguments. If the plasma density is low enough, an additional term containing the ion plasma frequency becomes important in the calculation of the lower hybrid frequency and

$$
\frac{1}{\omega_{l h}^{2}}=\frac{1}{\omega_{c e} \omega_{c i}}+\frac{1}{\left(\omega_{p i}^{2}+\omega_{c i}^{2}\right)} .
$$

where $\omega_{p i}$ is the ion plasma frequency. On axis, the plasma density is much higher and the first term in Equation (5.1) dominates. Therefore, towards the edge of the plasma where the density decreases, the ion plasma frequency term serves to lower the actual lower hybrid frequency. The arrow in Figure 70 shows to what values of RF frequency 
and magnetic field strength the resonance condition will shift in the plasma edge. If the perpendicular ion heating is a result of damping of the slow wave at the lower hybrid resonance, the maximum ion heating should occur at RF frequencies and magnetic field strengths such that the RF frequency is below the on-axis lower hybrid frequency because the slow wave only exists in the plasma edge [Shamrai and Taranov, 1996; Arnush and Chen, 1997; Chen and Arnush, 1997]. Thus, the RF frequencies and magnetic field strengths at which the maximum perpendicular ion heating occurs directly support the conclusion that the slow wave plays a key role in downstream ion heating in helicon sources.
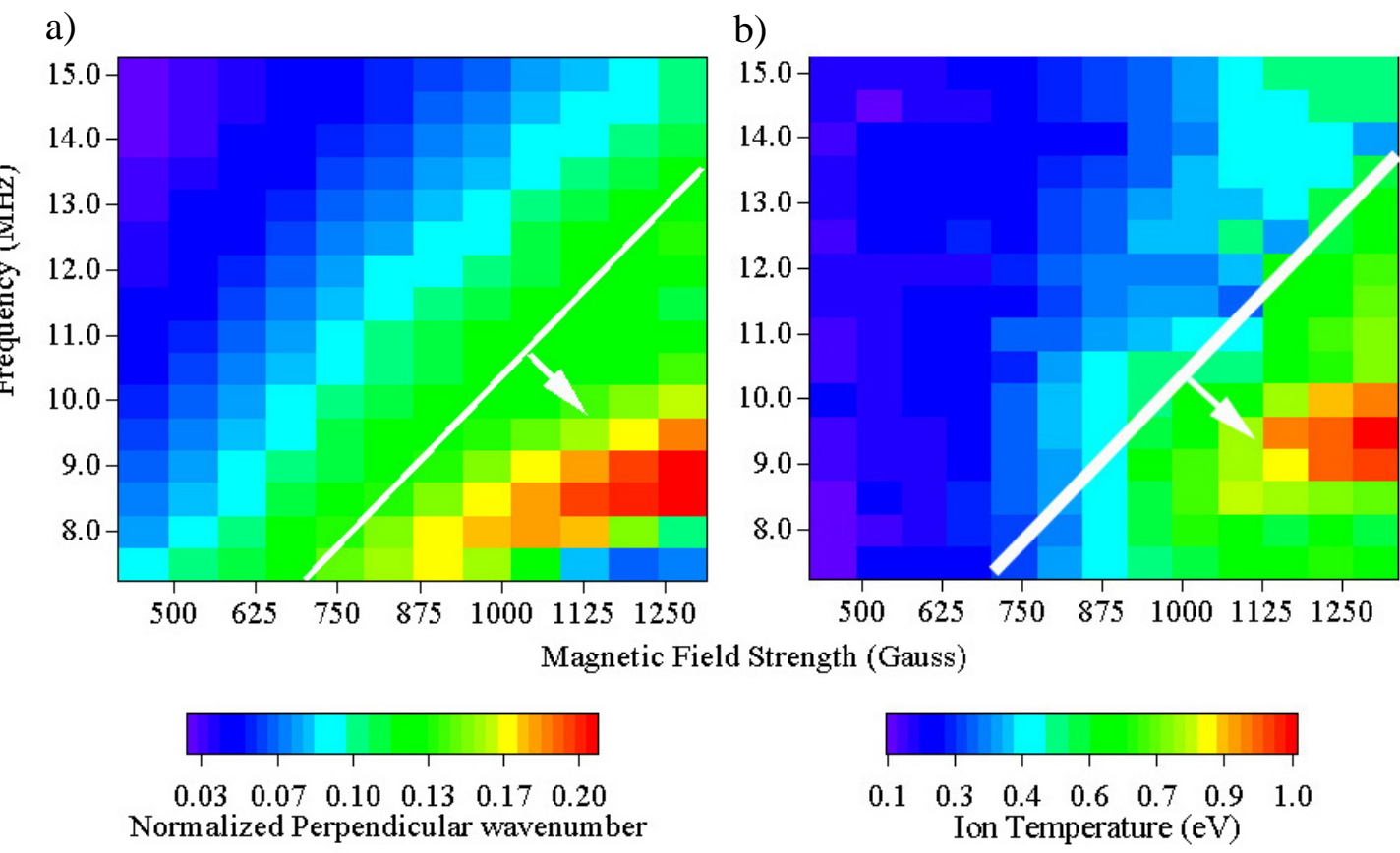

$\begin{array}{ccc}0.03 & 0.07 & 0.10 \\ \text { Normalized Perpendicular wavenumber }\end{array}$

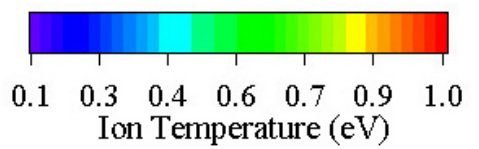

Figure 71: a) Normalized wave numbers, $k_{\perp} v_{t h i} / \omega$, from slow wave model. b) ion temperatures measured at the $35 \mathrm{~cm}$ location.

It could also be argued that the ion temperature data also provide direct evidence for the existence of the slow wave [Shamrai and Taranov, 1995; Cho, 1996; Chen and Arnush, 1997; Chen et al., 1997; Cho and Kwak, 1997; Borg and Boswell, 1998; Mouzouris and Scharer, 1998; Arnush, 2000; Shinohara and Yonekura, 2000; Blackwell et al., 2001; Schlüter et al., 2001; Shivarova and Tarnev, 2001]. The correlation of the ion heating with the lower hybrid resonance, the observations of perpendicular ion 
heating at the plasma edge, and the correspondence between the slow wave model and the ion temperature difference data all suggest that the slow wave is excited in helicon sources and propagates in one direction along the magnetic field at the plasma edge. 


\section{Chapter 5 References}

Arnush, D., The role of Trivelpiece-Gould waves in antenna coupling to helicon waves, Phys. Plasmas, 7, 3042 (2000).

Arnush, D., and Chen, F.F., Generalized theory of helicon waves. I. Normal modes., Phys. Plasmas, 4, 3411 (1997).

Balkey, M.M., Optimization of a Helicon Plasma Source for Maximum Density with Minimal Ion Heating, Ph. D. thesis, West Virginia University, Morgantown (2000).

Balkey, M.M., Boivin, R.F., Kline, J.L., and Scime, E.E., Ion heating and density production in helicon sources near the lower hybrid frequency, Plasma Sources Sci. Technol., 10, 284 (2001).

Blackwell, B.D., Madziwa, M.D., Arnush, D., and Chen, F.F., Direct Detection of Trivelpiece-Gould Modes in Helicon Discharges, Bulletin of the American Physical Society, 46, 63 (2001).

Borg, G.G., and Boswell, R.W., Power coupling to helicon and Trivelpiece-Gould modes in helicon sources, Phys. Plasmas, 5, 564 (1998).

Boswell, R.W., Perry, A.J., and Emami, M., Multipole confined diffusion plasma with RIPE source, J. Vac. Sci. Technol. A, 7, 3345 (1989).

Chen, F.F., Physics of helicon discharges, Phys. Plasmas, 3, 1783 (1996).

Chen, F.F., and Arnush, D., Generalized theory of helicon waves. I. Normal modes, Phys. Plasmas, 4, 3411 (1997).

Chen, F.F., and Boswell, R.W., Helicons-the past decade, IEEE Trans. Plasma Sci., 25, 1245 (1997).

Chen, F.F., Jiang, X., and Evans, J.D., Plasma injection with helicon sources, J. Vac. Sci. Technol. A, 18, 2108 (2000).

Chen, F.F., Jiang, X., Evans, J.D., Tynan, G., and Arnush, D., Low-field helicon discharges, Plasma Phys. Controlled Fusion, 39, 411 (1997).

Cho, S., The field and power absorption profiles in helicon plasma resonators, Phys. Plasmas, 3, 4268 (1996).

Cho, S., The role of the lower hybrid resonance in helicon plasmas, Phys. Plasmas, 7 , 417 (2000).

Cho, S., and Kwak, J.G., The effects of the density profile on the power absorption and the equilibrium density in helicon plasmas, Phys. Plasmas, 4, 4167 (1997).

Mouzouris, Y., and Scharer, J.E., Wave propagation and absorption simulations for helicon sources, Phys. Plasmas, 5, 4253 (1998).

Sakawa, Y., Takino, T., and Shoji, T., Contribution of slow waves on production of highdensity plasmas by $m=0$ helicon waves, Phys. Plasmas, 6, 4759 (1999).

Schlüter, H., Shivarova, A., and Tarnev, K., Effects of plasma-density inhomogeneity and collisions and their relation to maintenance of waveguide discharges by Trivelpiece-Gould modes: II. Numerical analysis, Plasma Sources Sci. Technol., 10, 267 (2001).

Shamrai, K.P., and Taranov, V.B., Resonances and anti-resonances of a plasma column in a helicon plasma source, Phys. Lett. A, 204, 139 (1995). 
Shamrai, K.P., and Taranov, V.B., Volume and surface RF power absorption in a helicon plasma source, Plasma Sources Sci. Technol., 5, 474 (1996).

Shinohara, S., and Yonekura, K., Discharge modes and wave structures using loop antennae in a helicon plasma source, Plasma Phys. Controlled Fusion, 42, 41 (2000).

Shivarova, A., and Tarnev, K., Effects of plasma-density inhomogeneity and collisions and their relation to maintenance of waveguide discharges by Trivelpiece-Gould modes: I. Resonance absorption, Plasma Sources Sci. Technol., 10, 260 (2001).

Skiff, F., Bachet, G., and Doveil, F., Ion dynamics in nonlinear electrostatic structures, Phys. Plasmas, 8, 3139 (2001).

Sudit, I.D., and Chen, F.F., Discharge equilibrium of a helicon plasma, Plasma Sources Sci. Technol., 5, 43 (1996).

Swanson, D.G., Plasma Waves, Academic Press, Inc., Boston, 1989.

Wong, K.L., and Ono, M., Parametric decay of lower-hybrid waves in the ACT-1 toroidal device, Phys. Rev. Lett., 47, 842 (1981).

Yun, S., Suwon, C., Tynan, G., and Hongyoung, C., Density enhancement near lower hybrid resonance layer in $m=0$ helicon wave plasmas, Phys. Plasmas, 8, 358 (2001).

Yun, S.M., and Chang, H.Y., Radial density profile change near lower hybrid frequency in $m=0$ helicon wave plasmas, Phys. Lett. A, 248, 400 (1998). 


\section{Chapter 6: Parametric Instabilities and Coupled Modes}

Whether it is the pure note of a tuning fork, the beat of a drum, or a propagating wave in a plasma, a normal mode of a physical system is an oscillatory motion at a single frequency that is associated with a particular displacement pattern of the medium. In many physical systems, coupling between different normal modes or between externally driven oscillations and normal modes can occur. If such coupling occurs as a result of the periodic variation in a physical parameter of the system, the coupling is called parametric coupling. Typically, parametric coupling in a plasma occurs when a plasma parameter contained in the dispersion relationship of a wave is varied. The modulation of the plasma parameter is often caused by another wave, producing a third beat wave. If the beat wave is unstable, it is called a parametric instability [Nishikawa and Wakatani, 2000].

Parametric coupling has been observed in a variety of laboratory plasmas. For example, during RF plasma heating in magnetic fusion experiments, parametric decay of the pump wave into normal modes of the plasma, e.g., lower hybrid and ion cyclotron waves, has been experimentally verified [Porkolab et al., 1972; Turner and Boyd, 1976; Porkolab, 1977; Imai et al., 1978; Albers et al., 1979; Wong and Ono, 1981]. Depending on the reason for externally driving an oscillation in a plasma, parametric decay that leads to the excitation of instabilities can either help or hinder attainment of the experimental objectives. In the case of RF heating, parametric decay instabilities can provide an additional mechanism for directly coupling pump wave energy into waves that damp on ions or electrons, thereby heating the plasma [Porkolab et al., 1972; Keen and Fletcher, 1973]. However, if the pump wave is intended to directly heat the plasma, parametric instabilities can reduce the pump wave power, thereby reducing the heating effectiveness of the pump wave [Wong and Ono, 1981]. Parametric instabilities can also cause anomalous particle transport, thereby reducing the confinement time and the heating effectiveness of the pump wave [McWilliams et al., 1983].

Not restricted to laboratory experiments, parametric decay processes play important roles in naturally occurring space phenomena and active space plasma experiments. During thunderstorms, lighting triggered whistler waves propagating in the ionosphere 
and magnetosphere have been observed to parametrically decay [Porkolab et al., 1973; Reeve and Boswell, 1976; Nenovski et al., 1979; Trakhtengerts and Rycroft, 1997]. During ionospheric modification experiments at the High Frequency Active Auroral Research Program (HAARP) facility in Alaska, parametric decay of the ground-baselaunched electromagnetic waves has also been observed [Wong, 1999]. In the early 1970's, Boswell, the inventor of the helicon source, investigated the parametric decay of large amplitude whistler waves in RF generated laboratory magnetoplasmas [Boswell, 1975b; Boswell, 1975a] to test models of parametric decay in space plasmas. Even though Boswell did not specifically use a helicon source for those experiments, the observations of parametric decay in HELIX plasmas reported in this work should have been expected given that helicon sources use an intense, whistler-like wave to generate the plasma.

In this Chapter, a review of the characteristics of the Mathieu equation provides a brief introduction to parametrically driven instabilities (Section 6.1). Parametric coupling and resonant decay instabilities arising from a pump wave and two normal modes in a plasma are described in Section 6.2. In Section 6.3, a short description of other types of parametrically driven plasma instabilities is presented.

\subsection{The Mathieu Equation}

One simple physical system that exhibits parametric instability is a simple pendulum with a variable length. Changing the length of the pendulum changes the frequency of oscillation of the pendulum, i.e. modifies the frequency of a normal, linear, mode of oscillation. Mathematically, the motion of a pendulum with a sinusoidally (in time) varying length is described by

$$
\frac{d^{2} X}{d t^{2}}+2 \gamma_{o} \frac{d X}{d t}+\left[\Omega_{o}^{2}\left(1+2 \varepsilon \cos \left(\omega_{o} t\right)\right)+\gamma_{o}^{2}\right] X=0
$$

where $\Omega_{o}$ is the natural frequency of the pendulum, $\omega_{o}$ is the frequency at which the length of the pendulum is varied, $\varepsilon$ is the amplitude of the variation in the pendulum length, and $\gamma_{o}$ is a damping term that represents any dissipation in the system. Equation (6.1) is the non-linear Mathieu equation, and has been the subject of considerable 
analysis [Swanson, 1989; Nishikawa and Wakatani, 2000]. The periodic solutions are the Mathieu functions [Blanch, 1970].

If the damping rate, $\gamma_{o}$, and the modulation amplitude, $\varepsilon$, are small $(|\varepsilon|<<1)$, the solutions to the Mathieu equations are unstable when $\Omega_{o} \simeq n \omega_{o} / 2(n=1,2,3, \ldots)$, the matching or resonant condition [Mima and Nishikawa, 1984]. Physically, when the beat frequencies at $\Omega_{o} \pm \omega_{o}$ equal another natural frequency of the system, e.g., $\Omega_{o} \pm \omega_{o}=-\Omega_{o}$, the modulation energy can be directly absorbed by the natural oscillation mode. If the growth rate of this driven natural oscillation exceeds the damping rate, an instability occurs. Thus, there is both a resonance condition and a threshold for exciting the instability [Swanson, 1989; Nishikawa and Wakatani, 2000]. In the weak modulation limit, the Mathieu equation can be solved using a perturbation method [Swanson, 1989]. The first step in obtaining the solution is to Fourier transform the equation, i.e., letting $X(t)=\int d \omega X(\omega) e^{-i \omega t} / 2 \pi$ gives,

$$
\left(-\omega^{2}-2 i \gamma_{o} \omega+\Omega_{o}^{2}+\gamma_{o}^{2}\right) X(\omega)+\varepsilon \Omega_{o}^{2}\left[X\left(\omega+\omega_{o}\right)+X\left(\omega-\omega_{o}\right)\right]=0 .
$$

or

$$
D(\omega) X(\omega)=-\varepsilon \Omega_{o}^{2}\left[X\left(\omega+\omega_{o}\right)+X\left(\omega-\omega_{o}\right)\right],
$$

where

$$
D(\omega)=\left(-\omega^{2}-2 i \gamma_{o} \omega+\Omega_{o}^{2}+\gamma_{o}^{2}\right) .
$$

$D(\omega)$ is the linear dispersion relationship whose solutions are given by $D(\omega)=0$. The right hand side of equation (6.3) represents the mode coupling of the natural frequency and the modulation frequency shifted by $\omega_{o}$ because of the modulation [Mima and Nishikawa, 1984]. Thus, solving for the behavior of the pendulum at some frequency $\omega$ requires the knowledge of the system behavior at $\omega \pm \omega_{o}$. Replacing $\omega$ by $\omega \pm m \omega_{o}$, where $m$ is again an integer, in Equation (6.3) gives the relationship between every mode and its nearest coupled modes:

$$
D\left(\omega \pm m \omega_{o}\right) X\left(\omega \pm m \omega_{o}\right)=-\varepsilon \Omega_{o}^{2}\left[X\left(\omega+[m \mp 1] \omega_{o}\right)+X\left(\omega-[m \pm 1] \omega_{o}\right)\right] .
$$


Equation (6.5) describes an infinite number of coupled mode equations that must be solved to obtain the behavior of the pendulum at a frequency of $\omega$. However, because the modulation amplitude is small, Equation (6.3) implies that the response of the system, $X(\omega)$, is small (on the order of $\varepsilon$ ) unless $\omega \pm \omega_{o}$ is close to $-\Omega_{o}$ or $\Omega_{o}$ (resonant excitation). Therefore, only those modes for which $\omega \pm \omega_{o}$ is close to $-\Omega_{o}$ or $\Omega_{o}$ are kept and the other modes neglected

As an example of mode coupling, consider the case of $\omega$ close to $\Omega_{o}$. To obtain a solution of the Mathieu equation at $\omega$, the effects of the nearest coupled modes $(m= \pm 1)$ must be included and therefore $\omega_{o} \simeq 2 \Omega_{o}$ or $\omega_{o} \simeq \Omega_{o}$. In this discussion, only the $\omega_{o} \simeq$ $2 \Omega_{o}$ case is considered as the solution provides a good example of a parametric instability. The solution for the $\omega_{o} \simeq \Omega_{o}$ case is reviewed in most texts [Mima and Nishikawa, 1984; Swanson, 1989; Nishikawa and Wakatani, 2000].

For $\omega_{o} \simeq 2 \Omega_{o}$ and $\omega \sim \Omega_{o}$, Equation (6.3) becomes

$$
D(\omega) X(\omega)=-\varepsilon \Omega_{o}^{2}\left[X\left(\omega-\omega_{o}\right)\right]
$$

As discussed above, the resonant term, $\omega-\omega_{o}=-\Omega_{o}$, dominates the response of the system and the non-resonant term, $\omega+\omega_{o}=3 \Omega_{o}$, can be neglected. To close the set of equations for $X(\omega)$ and $X\left(\omega-\omega_{o}\right)$, Equation (6.5) is evaluated at $m=-1$ to obtain $X\left(\omega-\omega_{o}\right)$ in terms of $X(\omega)$ :

$$
D\left(\omega-\omega_{o}\right) X\left(\omega-\omega_{o}\right)=-\varepsilon \Omega_{o}^{2}\left[X(\omega)+X\left(\omega-2 \omega_{o}\right)\right]
$$

Again, the non-resonant term is neglected and Equation (6.7) reduces to

$$
D\left(\omega-\omega_{o}\right) X\left(\omega-\omega_{o}\right)=-\varepsilon \Omega_{o}^{2} X(\omega)
$$

Multiplying both sides by $D(\omega)$ and substituting Equation (6.6) for $D(\omega) X(\omega)$ on the right hand side

$$
\begin{aligned}
D(\omega) D\left(\omega-\omega_{o}\right) X\left(\omega-\omega_{o}\right) & =-\varepsilon \Omega_{o}^{2} D(\omega) X(\omega) \\
& =\varepsilon^{2} \Omega_{o}^{4} X\left(\omega-\omega_{o}\right) .
\end{aligned}
$$


Dividing both sides by $X\left(\omega-\omega_{0}\right)$,

$$
D(\omega) D\left(\omega-\omega_{o}\right)=\varepsilon^{2} \Omega_{o}^{4} .
$$

From Equation (6.4), $D(\omega)$ is

$$
\begin{aligned}
D(\omega) & =\left(-\omega^{2}-2 i \gamma_{o} \omega+\Omega_{o}^{2}+\gamma_{o}^{2}\right) \\
& =-\left(\omega+\Omega_{o}-i \gamma_{o}\right)\left(\omega-\Omega_{o}+i \gamma_{o}\right)
\end{aligned}
$$

and $D\left(\omega-\omega_{o}\right)$ can be written as

$$
\begin{aligned}
& D\left(\omega-\omega_{o}\right)=\left(-\left[\omega-\omega_{o}\right]^{2}-2 i \gamma_{o} \omega+\Omega_{o}^{2}+\gamma_{o}^{2}\right) \\
& \quad=-\left(\omega-\omega_{o}+\Omega_{o}-i \gamma_{o}\right)\left(\omega+\omega_{o}-\Omega_{o}+i \gamma_{o}\right) .
\end{aligned}
$$

Since $\omega \simeq \Omega_{o}$ and $\omega-\omega_{o} \simeq-\Omega_{o}$, Equations (6.11) and (6.12) can be approximated as

$$
\begin{aligned}
& D(\omega) \approx-2 \Omega_{o}\left(\omega-\Omega_{o}+i \gamma_{o}\right) \\
& D(\omega) \approx 2 \Omega_{o}\left(\omega-\omega_{o}+\Omega_{o}+i \gamma_{o}\right)
\end{aligned}
$$

and Equation (6.10) becomes

$$
\left(\omega-\Omega_{o}+i \gamma_{o}\right)\left(\omega-\omega_{o}+\Omega_{o}+i \gamma_{o}\right)+\frac{1}{4} \varepsilon^{2} \Omega_{o}^{2}=0 .
$$

From the matching condition, $\omega_{o} \simeq 2 \Omega_{o}$, a frequency mismatch is defined as $\Delta \equiv \omega_{o}-2 \Omega_{o}$. For analysis purposes, it is convenient to write the frequency as $\omega \equiv \Omega_{o}+\delta$ $+i \gamma$. In this form, the frequency is centered around the natural frequency since $\omega \simeq \Omega_{o}$ and $\delta$ is the frequency shift from the natural frequency. The damping rate of the mode is given by the imaginary part of $\omega, \gamma$. With these definitions, Equation (6.15) can be separated into real and imaginary parts [Swanson, 1989]:

$$
\begin{gathered}
\delta(\delta-\Delta)-\left(\gamma-\gamma_{o}\right)^{2}+\frac{1}{4} \varepsilon^{2} \Omega_{o}^{2}=0 \\
(2 \delta-\Delta)\left(\gamma-\gamma_{o}\right)=0 .
\end{gathered}
$$

Equation (6.17) has two solutions, $\delta=\Delta / 2$ and $\gamma=-\gamma_{o}$. The first solution, $\delta=\Delta / 2$, is the "locked" solution [Swanson, 1989] and is characterized by an oscillation frequency that is independent of the natural frequency, i.e., $\operatorname{Re}(\omega)=\omega_{o} / 2$. According to Equation (6.16), the growth rate of the locked solution is 


$$
\gamma=-\gamma_{o} \pm \frac{1}{2} \sqrt{\varepsilon^{2} \Omega_{o}^{2}-\Delta^{2}}
$$

In the absence of dissipation, $\gamma_{o}=0$, finite growth of the frequency locked parametric instability $(\gamma>0)$ requires, $\varepsilon>\Delta^{2} / \Omega_{o}$, i.e., a minimum modulation amplitude is required. With dissipation $\left(\gamma_{o}>0\right)$, even larger modulation amplitudes are required:

$$
\varepsilon>\sqrt{\left(\Delta^{2}+4 \gamma_{o}^{2}\right)} / \Omega_{o}
$$

The minimum modulation amplitude threshold occurs at exact matching, $\Delta=0$ [Swanson, 1989], and is $\varepsilon_{\min }=2 \gamma_{o} / \Omega_{o}$. The growth rate of the frequency locked parametric instability at the minimum threshold is $\gamma=-\gamma_{o}+\varepsilon / 2 \Omega_{o}$.

The second solution to Equation (6.17), the $\gamma=-\gamma_{o}$ case, is known as the damped solution [Swanson, 1989]. Since $\gamma$ is always negative, the solutions are damped oscillations with a frequency shift. According to Equation (6.16), the frequency of the damped solution is given by

$$
\delta=\frac{1}{2}\left(\Delta \pm \sqrt{\Delta^{2}-\varepsilon^{2} \Omega_{o}^{2}}\right)
$$

As was seen in the frequency locked case, a minimum modulation amplitude of $\varepsilon>$ $\Delta^{2} / \Omega_{o}$ is required to excite damped, parametrically driven oscillations. As this brief discussion demonstrated, minor modifications to a relatively simple physical system, a pendulum, can result in parametric coupling between normal modes of the system and the

parametric excitation of instabilities. Note that the parametric instability was most easily excited when the frequency mismatch was zero, i.e., when the modulation frequency was a harmonic of the system's natural oscillation frequency.

\subsection{Parametric Coupling and Instabilities in Plasmas}

A technique known as coupled mode analysis [Mima and Nishikawa, 1984; Nishikawa and Wakatani, 2000] has been developed to investigate the nature of the interaction between normal modes and driven oscillations in plasmas. In the previous example, the physical system had a single frequency. In a plasma, a variety of normal modes, each 
with different oscillation frequencies, can co-exist. Consider a plasma with an externally driven oscillation, a pump wave, given by

$$
Z(\vec{r}, t)=2 Z_{o} \cos \left(\vec{k} \cdot \vec{r}-\omega_{o} t\right)
$$

In the plasma, there are two normal modes with eigenfrequencies $\omega_{1}(\mathbf{k})$ and $\omega_{2}(\mathbf{k})$, where $\omega_{1}(\mathbf{k})<\omega_{2}(\mathbf{k})$ and $\boldsymbol{k}$ is the wavevector. As in the pendulum example, the Fourier components of the responses for the modes are given by $X_{1}$ and $X_{2}$. The coupling equations are assumed to be of the form

$$
\begin{aligned}
& {\left[\frac{\partial}{\partial t^{2}}+2 \gamma_{1} \frac{\partial}{\partial t}+\omega_{1}^{2}(\vec{k})+\gamma_{1}^{2}\right] X_{1}(\vec{k}, t)=} \\
& Z_{o}\left[\lambda_{+} X_{2}\left(\vec{k}+\vec{k}_{o}, t\right) e^{-i \omega_{o} t}+\lambda_{-} X_{2}\left(\vec{k}-\vec{k}_{o}, t\right) e^{i \omega_{o} t}\right] \\
& {\left[\frac{\partial}{\partial t^{2}}+2 \gamma_{2} \frac{\partial}{\partial t}+\omega_{2}^{2}(\vec{k} \pm \vec{k})+\gamma_{2}^{2}\right] X_{2}\left(\vec{k} \pm \vec{k}_{o}, t\right)=} \\
& Z_{o}\left[\mu_{+} X_{1}(\vec{k}, t) e^{-i \omega_{o} t}+\mu_{-} X_{1}(\vec{k}, t) e^{+i \omega_{o} t}\right]
\end{aligned}
$$

where $Z_{o}$ is the pump amplitude, $\gamma_{1}$ and $\gamma_{2}$ are the linear damping rates of each normal mode and $\lambda_{ \pm}$and $\mu_{ \pm}$are the coupling coefficients [Mima and Nishikawa, 1984]. The coupling of the two modes depends on the coupling coefficients as well as the pump amplitude [Mima and Nishikawa, 1984]. Writing Equation (6.22) and Equation (6.23) in the Laplace transformed representation yields an infinite set of linked equations that describe the relationships between the normal modes and sidebands at the beat frequencies: $X_{1}\left(\boldsymbol{k} \pm n \boldsymbol{k}_{o}, \omega \pm n \omega_{o}\right)$ and $X_{2}\left(\boldsymbol{k} \pm m \boldsymbol{k}_{o}, \omega \pm m \omega_{o}\right)$ with $(n, m=0, \pm 1$, $\pm 2, \ldots)[$ Mima and Nishikawa, 1984]. As was done for the Mathieu equation, only three modes are kept, $n=0$ and $m= \pm 1$, i.e., $X_{1}(\boldsymbol{k}, \omega)$ and $X_{2}\left(\boldsymbol{k} \pm \boldsymbol{k}_{o}, \boldsymbol{\omega} \pm \omega_{o}\right)$ [Mima and Nishikawa, 1984]. In the weak coupling limit, the linear dispersion relations for the two modes,

$$
D_{1}(\vec{k}, \omega)=\left(\omega+i \gamma_{1}\right)^{2}-\omega_{1}^{2}(\vec{k})
$$

and

$$
D_{2}\left(\vec{k} \pm \vec{k}_{o}, \omega \pm \omega_{o}\right)=\left(\omega \pm \omega_{o}+i \gamma_{2}\right)^{2}-\omega_{2}^{2}\left(\vec{k} \pm \vec{k}_{o}\right)
$$


are nearly satisfied and can be used to rewrite the coupled mode equations:

$$
D_{1}(k, \omega) X_{1}(\vec{k}, \omega)=Z_{o}\left[\lambda_{+} X_{2}\left(\vec{k}+\vec{k}_{o}, \omega+\omega_{o}\right)+\lambda_{-} X_{2}\left(\vec{k}-\vec{k}_{o}, \omega-\omega_{o}\right)\right]
$$

and

$$
D_{2}\left(\vec{k} \pm \vec{k}_{o}, \omega \pm \omega_{o}\right) X_{2}\left(\vec{k} \pm \vec{k}_{o}, \omega \pm \omega_{o}\right)=Z_{o} \mu_{ \pm} X_{1}(\vec{k}, \omega) .
$$

Rearranging Equation (6.27)

$$
X_{2}\left(\vec{k} \pm \vec{k}_{o}, \omega \pm \omega_{o}\right)=\frac{Z_{o} \mu_{ \pm} X_{1}(\vec{k}, \omega)}{D_{2}\left(\vec{k} \pm \vec{k}_{o}, \omega \pm \omega_{o}\right)},
$$

and substituting the result into equation (6.26) for the different wavevector and frequency values yields

$$
D_{1}(k, \omega) X_{1}(\vec{k}, \omega)=Z_{o}^{2}\left[\frac{\lambda_{+} \mu_{+} X_{1}(\vec{k}, \omega)}{D_{2}\left(\vec{k}+\vec{k}_{o}, \omega+\omega_{o}\right)}+\frac{\lambda_{-} \mu_{-} X_{1}(\vec{k}, \omega)}{D_{2}\left(\vec{k}-\vec{k}_{o}, \omega-\omega_{o}\right)}\right]
$$

Dividing by the mode response, $X_{1}$, gives a compact form of the dispersion relation [Mima and Nishikawa, 1984]:

$$
1=\frac{Z_{o}^{2}}{D_{1}(k, \omega)}\left[\frac{\lambda_{+} \mu_{+}}{D_{2}\left(\vec{k}+\vec{k}_{o}, \omega+\omega_{o}\right)}+\frac{\lambda_{-} \mu_{-}}{D_{2}\left(\vec{k}-\vec{k}_{o}, \omega-\omega_{o}\right)}\right],
$$

where $\lambda_{ \pm}$and $\mu_{ \pm}$are now assumed to be real constants. In the limit of weak coupling, $\left|Z_{\mathrm{o}} \lambda_{ \pm} \mu_{ \pm}\right| \ll<1$, the numerators of both terms of Equation (6.30) are very small. Thus, the only possible solutions require some or all of the dispersion relations to be approximately satisfied. The two possible solutions are [Mima and Nishikawa, 1984; Nishikawa and Wakatani, 2000]:

a) $D_{1}(\boldsymbol{k}, \omega) \simeq 0$ and $D_{2}\left(\boldsymbol{k}+\boldsymbol{k}_{o}, \omega+\omega_{o}\right) \simeq 0$ or $D_{2}\left(\boldsymbol{k}-\boldsymbol{k}_{o}, \omega-\omega_{o}\right) \simeq 0$

b) $D_{2}\left(\boldsymbol{k}+\boldsymbol{k}_{o}, \omega+\omega_{o}\right) \simeq 0$ and $D_{2}\left(\boldsymbol{k}-\boldsymbol{k}_{o}, \omega-\omega_{o}\right) \simeq 0$.

Because the roots of the $D_{2}$ dispersion relation do not depend on $D_{1}(\boldsymbol{k}, \boldsymbol{\omega})$, i.e. there is no coupling between $D_{1}(\boldsymbol{k}, \omega)$ and $D_{2}\left(\boldsymbol{k} \pm \boldsymbol{k}_{o}, \omega \pm \omega_{o}\right)$, the second solution describes non- 
resonant coupling between the normal modes. Such non-resonant processes can lead to a number of instabilities including the frequency locked parametric instability seen in the Mathieu equation example, the oscillating two-stream instability [Nishikawa, 1968], and the quasi-reactive mode instability [Nishikawa, 1968]. In all cases, growth of the nonresonant instabilities does not require the sum of the normal mode frequencies to equal to the pump frequency. Since, as will be shown in Chapter 7, the experimental data suggests resonant mode coupling occurs in HELIX (the mode frequencies sum to the pump frequency), the non-resonant coupling case will not be explored further in this discussion. Instead, the characteristics of resonant mode coupling will be investigated by using the mode coupling equations to derive relationships between the normal mode characteristics and the pump wave.

To simplify the algebra, the linear damping rates of the two normal modes are set to zero, $\gamma_{1}=\gamma_{2}=0$, and the pump frequency is assumed to be close to the natural frequency of the higher frequency normal mode, $\omega_{0} \simeq \omega_{2} \gg \omega_{1}$. With these assumptions, the dispersion relation for the higher frequency mode, Equation (6.25), can be written as [Mima and Nishikawa, 1984; Nishikawa and Wakatani, 2000]

$$
D_{2}\left(\vec{k} \pm \vec{k}_{o}, \omega \pm \omega_{o}\right) \simeq \pm 2 \omega_{o}[(\omega-\alpha) \pm \delta],
$$

where

$$
\alpha=\frac{\omega_{2}\left(\vec{k}+\vec{k}_{o}\right)-\omega_{2}\left(\vec{k}-\vec{k}_{o}\right)}{2}
$$

and

$$
\delta=\omega_{o}-\frac{\left(\omega_{2}\left(\vec{k}+\vec{k}_{o}\right)+\omega_{2}\left(\vec{k}-\vec{k}_{o}\right)\right)}{2} .
$$

Rewriting Equation (6.30) as

$$
\begin{aligned}
& D_{1}(k, \omega) D_{2}\left(\vec{k}+\vec{k}_{o}, \omega+\omega_{o}\right) D_{2}\left(\vec{k}-\vec{k}_{o}, \omega-\omega_{o}\right)= \\
& Z_{o}^{2}\left[\lambda_{+} \mu_{+} D_{2}\left(\vec{k}-\vec{k}_{o}, \omega-\omega_{o}\right)+\lambda_{-} \mu_{-} D_{2}\left(\vec{k}+\vec{k}_{o}, \omega+\omega_{o}\right)\right]
\end{aligned}
$$

and then using Equation (6.31) yields 


$$
\begin{aligned}
-4 \omega_{o}^{2} D_{1}(k, \omega)[(\omega-\alpha) & +\delta][(\omega-\alpha)-\delta]= \\
& -2 \omega_{o} Z_{o}^{2}\left[\lambda_{+} \mu_{+}[(\omega-\alpha)-\delta]-\lambda_{-} \mu_{-}[(\omega-\alpha)+\delta]\right] .
\end{aligned}
$$

According to [Mima and Nishikawa, 1984; Nishikawa and Wakatani, 2000], for many cases of interest the coupling parameters $\lambda_{+} \mu_{+}$and $\lambda_{-} \mu_{-}$are real and identical. Under the constraint that $Z_{\mathrm{o}}{ }^{2} \lambda_{+} \mu_{+}=Z_{\mathrm{o}}{ }^{2} \lambda_{-} \mu_{-} \equiv \varepsilon^{2} \omega_{\mathrm{o}}{ }^{2} \omega_{1}{ }^{2}$ (where $\varepsilon$ is a small dimensionless parameter that represents the coupling strength), Equation (6.35) becomes

$$
\begin{aligned}
& -4 \omega_{o}^{2} D_{1}(k, \omega)[(\omega-\alpha)+\delta][(\omega-\alpha)-\delta]= \\
& 2 \varepsilon^{2} \omega_{1}^{2} \omega_{o}^{3}[[(\omega-\alpha)-\delta]-[(\omega-\alpha)+\delta]] .
\end{aligned}
$$

Substituting Equation (6.24) for the low frequency mode dispersion relation, Equation (6.36) reduces to

$$
\left[\omega^{2}-\omega_{1}^{2}\right]\left[(\omega-\alpha)^{2}-\delta^{2}\right]=-\varepsilon^{2} \omega_{o} \omega_{1}^{2} \delta
$$

This bi-quadratic equation has four different roots for $\omega$. At low pump power $(\varepsilon<<1)$, if $\omega \approx \pm \omega_{1}$ and $\omega \approx \alpha \pm \delta$, Equation (6.37) is satisfied. If the positive terms are used, $\omega \approx \omega_{1} \approx \alpha+\delta$, Equation (6.37) is equivalent to the resonance condition

$$
\omega_{o}\left(\vec{k}_{o}\right)=\omega_{1}(\vec{k})+\omega_{2}\left(\vec{k}-\vec{k}_{o}\right) .
$$

This well-known resonance, or frequency matching condition, for resonant decay coupling is essentially conservation of energy for the pump wave, the upper frequency normal mode, and the parametrically driven lower frequency normal mode. Since no damping of the normal modes was assumed in this analysis, the parametrically driven wave in this case is unstable for finite pump amplitudes.

Although a great many approximations were required to arrive at the simple resonance condition, a more complete treatment does not alter the fundamental characteristics of the parametric coupling process. For example, in the weak damping case $\left(0<\gamma_{1}<<\omega_{1}\right.$ and $0<$ $\left.\gamma_{2}<<\omega_{2}\right)$ the damping terms can be incorporated into Equation (6.37) by replacing $\omega$ with $\omega+i \gamma_{1}$ and $\omega+i \gamma_{2}$ giving [Mima and Nishikawa, 1984]

$$
\left[\left(\omega+i \gamma_{1}\right)^{2}-\omega_{1}^{2}\right]\left[\left(\omega+i \gamma_{2}-\alpha\right)^{2}-\delta^{2}\right]=-\varepsilon^{2} \omega_{o} \omega_{1}^{2} \delta
$$


If the frequency is divided into real and imaginary parts, $\omega=\omega_{r}+i \gamma$, Equation (6.39) can be solved for the real frequency and growth rate of the parametrically driven wave. Using the resonant decay condition, the maximum growth rate is [Mima and Nishikawa, 1984]

$$
\left(\gamma_{\max }+\gamma_{1}\right)\left(\gamma_{\max }+\gamma_{2}\right)=\varepsilon^{2} \omega_{o} \omega_{1}^{2} / 4
$$

and the threshold for instability is $\varepsilon_{\min }=2 \sqrt{\gamma_{1} \gamma_{2} / \omega_{0} \omega_{1}}$. The instability threshold condition is essentially the same result that was obtained for the Mathieu equation. More specific calculations require additional information about the wave number of the pump wave and the details of the linear dispersion relations for the plasma waves. For example, in the case of an electromagnetic pump wave, a high frequency electrostatic, electron plasma wave, and a low frequency electrostatic, ion acoustic wave, the wave numbers of the waves must also satisfy $\boldsymbol{k}_{\mathrm{o}}=\boldsymbol{k}_{1}+\boldsymbol{k}_{2}$ for resonant three mode coupling to occur.

\subsection{Other Parametrically Driven Instabilities}

Even though parametric coupling between a pump wave and two normal modes is common in plasmas, other parametrically driven phenomena occur. In helicon sources, one parameter that can be significantly affected by the propagation of the helicon wave is the transverse electron current. Since the helicon wave's electric field component, $\mathbf{E}_{\mathbf{1}}$, is perpendicular to the applied magnetic field, $\mathbf{B}_{\mathbf{0}}$, the electrons drift at the $\mathbf{E}_{\mathbf{1}} \times \mathbf{x} \mathbf{B}_{\mathbf{o}}$ velocity as long as the helicon wave frequency is much less than the electron cyclotron frequency. The ions cannot $\mathbf{E}_{\mathbf{1}} \times \mathbf{B}_{\mathbf{0}}$ drift because the wave frequency is much larger than the ion cyclotron frequency and the ions are effectively stationary. With the electrons drifting and the ions stationary, a net transverse current develops. The electron drift is oscillatory because the transverse electric field oscillates in time and an alternating transverse current can excite instabilities. There are three types of transverse current driven, parametric instabilities that could play important roles in helicon sources: lower hybrid [Sizonenko, 1976; Stepanov, 1996], ion cyclotron [Kitsenko et al., 1974b; Kitsenko et al., 1974a; Korzh et al., 1984; Korzh et al., 1989] [Stepanov, 1996], and ion acoustic [Akhiezer et al., 1997; Akhiezer et al., 1998; Akhiezer et al., 2000] instabilities. These 
instabilities are parametrically driven by changes in a key element of a particular dispersion relation, but do not necessarily involve a three-mode resonant decay process.

The first of these transverse current instabilities, the lower hybrid instability, occurs when the electron drift speed, $u \sim\left|E_{1} / B_{\mathrm{o}}\right|$, is between the ion and electron thermal speeds, $\mathrm{v}_{\text {thi }} \ll<u<\mathrm{v}_{\text {the }}[$ Stepanov, 1996]. The linear dispersion relationship is

$$
1+\frac{\omega_{p e}^{2}}{\omega_{c e}^{2}}-\frac{\omega_{p e}^{2}}{\left(\omega-k u_{e}\right)^{2}}\left(\frac{k_{\|}^{2}}{k^{2}}\right)-\frac{\omega_{p i}^{2}}{\left(\omega-k u_{i}\right)^{2}}=0,
$$

where $\omega_{p e}$ and $\omega_{p i}$ are the electron and ion plasma frequencies, $u_{e}$ and $u_{i}$ are the electron and ion drift velocities, and $\omega_{c e}$ is the electron cyclotron frequency [Stepanov, 1996]. The typical growth rates for the lower hybrid instabilities are on the order of the lower hybrid frequency, $\gamma \sim \omega_{l h}$. The lower hybrid instability can anisotropically heat ions and the heating rate is [Sizonenko, 1976; Stepanov, 1996]

$$
\frac{\partial T_{\perp i}}{\partial t}=\frac{\omega_{c i}^{2}}{\omega_{l h}} \cdot \frac{m_{i} u_{e}}{2}
$$

where $T_{\perp i}$ is the perpendicular ion temperature, and $\omega_{c i}$ is the ion cyclotron frequency, lower hybrid instabilities parametrically driven by the helicon wave could be important for ion heating in helicon sources.

Another parametric instability associated with electromagnetic waves is the beam-like kinetic ion cyclotron, the ion Bernstein, instability. The kinetic ion cyclotron wave is also parametrically coupled to the helicon wave through the transverse electron currents and can be excited when the electron drift velocity is less than the ion thermal speed, $\mathrm{v}_{t h i}$ $>>u$. In the short wavelength limit $\left(k_{\perp} \rho_{i}>1\right)$, the wave frequencies are close to the cyclotron harmonics [Stepanov, 1996]

$$
\omega=n \omega_{c i}+\delta \omega
$$

where $\delta \omega=\alpha n \omega_{c i} / k_{\perp} \rho_{i}, \alpha \sim 1, \rho_{i}$ is the ion gyroradius, and $\omega_{c i}$ is the ion cyclotron frequency. Excitation of the cyclotron waves is due to resonant electrons moving along the applied magnetic field with [Stepanov, 1996]

$$
\mathrm{v}_{e \|}=\frac{\omega+k \cdot u}{k_{\|}} \sim \mathrm{v}_{t h e},
$$


where $\mathrm{v}_{\mathrm{e} \|}$ is the electron's parallel velocity, and $u$ is the electron drift speed. Typical wave numbers for the instability are [Stepanov, 1996]

$$
k \sim \frac{\mathrm{v}_{t h i}}{\rho_{i} u}>>1 \quad \frac{k_{\|}}{k}=\frac{u}{\mathrm{v}_{\text {the }}}<<1 .
$$

This parametrically driven instability propagates primarily perpendicular to the magnetic field. Since the wave frequency is near the ion cyclotron frequency, the wave can supply energy to the ions that is subsequently thermalized by collisions or wave-particle scattering, thereby increasing the perpendicular ion temperature. If the cyclotron wave scattering by free ions is large enough, the kinetic ion cyclotron instability becomes nonlinear. The nonlinear stage has been examined by Mikhailenko [Mikhailenko and Stepanov, 1981; Mikhailenko and Stepanov, 1986].

The third parametric instability excited by transverse currents is the short wavelength, ion acoustic, parametric instability. Short wavelength ion acoustic waves at the RF pump frequency can be excited when the electron drift speed, $u$, is larger than the ion acoustic speed, $c_{s} \sim\left(\gamma Z k_{b} T_{e} / m_{i}\right)^{1 / 2}$ for $T_{e}>T_{i}$ where $c_{s}$ is the sound speed, $\gamma$ is the adiabatic constant, $Z$ is the ionization number, $m_{i}$ is the ion species mass, $k_{b}$ is Boltzmann's constant, $T_{e}$ is the electron temperature and $T_{i}$ is the ion temperature [Akhiezer et al., 1997; Akhiezer et al., 1998; Akhiezer et al., 2000]. Since typical helicon sources operate in the tens of megahertz range and the dispersion relationship for sound waves is $\omega=k c_{s}$ where $c_{s}$ is the sound speed, the wavelength of the sound waves must be very small, i.e. $k_{\perp} \rho_{i} \gg k_{\perp} \rho_{e} \gg 1$. The acoustic instability differs from the ion cyclotron instability because the instability wavelengths are so much smaller than the ion gyroradius that the ions are effectively unmagnetized. The short wavelength, ion acoustic instability also propagates nearly perpendicular to the applied magnetic field. When nonlinear saturation occurs due to scattering of the ion sound waves, perpendicular ion heating can occur [Akhiezer et al., 1998]. When electrons are scattered from the short wavelength waves, the effective collision frequency for the electrons increases. Such an increase in electron collisionality has been proposed as an explanation for the observed damping of the helicon wave and the efficient RF power absorption of helicon sources producing the 
high plasma densities of helicon sources [Akhiezer et al., 1998]. In both the ion and the electron case, energy from the RF pump wave is transformed directly into particle thermal energy.

All three instabilities are parametrically driven by transverse currents from the $\mathbf{E}_{\mathbf{1}} \times \mathbf{B}_{\mathbf{0}}$ drift of electrons in externally driven RF waves. All three are examples of parametric coupling in plasmas that is not a resonant parametric decay process. For helicon sources, these three instabilities could result in ion heating or enhanced plasma densities. 


\section{Chapter 6 References}

Akhiezer, A.I., Mikhailenko, V.S., Ol'shansky, V.V., and Stepanov, K.N., Parametric turbulence-sustained gas discharges, Plasma Physics Reports, 26, 575 (2000).

Akhiezer, A.I., Mikhailenko, V.S., and Stepanov, K.N., Ion sound plasma turbulence with a transverse current in a magnetic field, Ukrayins'kyi Fizychnyi Zhurnal, 42, 990 (1997).

Akhiezer, A.I., Mikhailenko, V.S., and Stepanov, K.N., Ion-sound parametric turbulence and anomalous electron heating with application to helicon plasma sources, Phys. Lett. A, 245, 117 (1998).

Albers, E., Krause, K., and Schluter, H., Parametric excitation of backward ion cyclotron wave with a pump in the lower hybrid range, Plasma Physics, 21, 193 (1979).

Blanch, G., Mathieu Functions, in Handbook of Mathematical Functions, edited by M. Abramowitz, and I.A. Stegun, pp. 721, Dover Publications, Inc., New York, 1970.

Boswell, R.W., Measurements of the far-field resonance cone for whistler mode waves in a magnetoplasma, Nature, 258, 58 (1975a).

Boswell, R.W., Non-convective parametric instability associated with whistler wave resonance cone, Phys. Lett. A, 55A, 93 (1975b).

Imai, T., Nagashima, T., Yamamoto, T., Uehara, K., Konoshima, S., Takeuchi, H., Yoshida, H., and Fujisawa, N., Parametric instabilities in lower hybrid frequency heating of a Tokamak, Phys. Rev. Lett., 43, 586 (1978).

Keen, B.E., and Fletcher, W.H.W., Plasma heating effects in the presence of a parametric decay instability, Journal of Physics A (Mathematical and General), 6, L24 (1973).

Kitsenko, A.B., Panchenko, V.I., and Stepanov, K.N., Electron-acoustic and ion cyclotron parametric instabilities in an alternating electric field. I, Soviet Physics Technical Physics, 18, 905 (1974a).

Kitsenko, A.B., Panchenko, V.I., and Stepanov, K.N., Electron-acoustic and ion cyclotron parametric instabilities of a plasma in an alternating electric field. II, Soviet Physics Technical Physics, 18, 911 (1974b).

Korzh, A.F., Lapshin, V.I., and Stepanov, K.N., Kinetic ion-cyclotron parametric instability, Soviet Journal of Plasma Physics, 10, 747 (1984).

Korzh, A.F., Mikhailenko, V.S., and Stepanov, K.N., Parametric ion cyclotron turbulence of plasma in nonuniform electric and magnetic fields of an MHD wave, Soviet Journal of Plasma Physics, 15, 413 (1989).

McWilliams, R., Hill, D.N., Wolf, N.S., and Rynn, N., Cross-field ion transport and heating due to parametric decay of lower hybrid waves, Phys. Rev. Lett., 50, 836 (1983).

Mikhailenko, V.S., and Stepanov, K.N., Saturation of the electron cyclotron instability and turbulent heating of a plasma with a transverse current, Fizika Plazmy, 7, 365 (1981).

Mikhailenko, V.S., and Stepanov, K.N., Comments on the nonlinear theory of ion cyclotron instability of a plasma with transverse current, Plasma Phys. Controlled Fusion, 28, 613 (1986). 
Mima, K., and Nishikawa, K., Parametric Instabilities and Wave Dissipation in Plasmas, in Handbook of Plasma Physics, edited by M.N. Rosenbluth, and R.Z. Sagdeev, pp. 451, North-Holland Physics Publishing, Amsterdam, 1984.

Nenovski, P., Shukla, P.K., Tagare, S.G., and Zhelyazkov, I., Parametric decay of nonducted whistler-mode signal, (1979).

Nishikawa, K., J. Phys. Soc. Jpn., 24, 916 (1968).

Nishikawa, K., and Wakatani, M., Plasma Physics: Basic Theory with Fusion Applications, Springer, Berlin, 2000.

Porkolab, M., Parametric instabilities due to lower-hybrid radio frequency heating of Tokamak plasmas, Phys. Fluids, 20, 2058 (1977).

Porkolab, M., Arunasalam, V., and Ellis, R.A., Jr., Parametric instability and anomalous heating due to electromagnetic waves in plasma, Phys. Rev. Lett., 29, 1438 (1972).

Porkolab, M., Arunasalam, V., and Grek, B., Parametric instabilities and anomalous absorption and heating in magnetoplasmas, (1973).

Reeve, C.D., and Boswell, R.W., Parametric decay of whistlers-a possible source of precursors, Geophysical Research Letters, 3, 405 (1976).

Sizonenko, V.L., Problems of Plasma Theory, in Procedures of the 2nd International Conference on Plasma Physics, pp. 188, Kiev, 1976.

Stepanov, K.N., Nonlinear parametric phenomena in plasma during radio frequency heating in the ion cyclotron frequency range, Plasma Phys. Controlled Fusion, 38, A13 (1996).

Swanson, D.G., Plasma Waves, Academic Press, Inc., Boston, 1989.

Trakhtengerts, V.Y., and Rycroft, M.J., A new parametric reflection mechanism for ducted whistlers and an explanation of precursors, Journal of Atmospheric and Solar Terrestrial Physics, 59, 1865 (1997).

Turner, J.G., and Boyd, T.J.M., Parametric decay of whistler waves, Physica Scripta, 14, 175 (1976).

Wong, A.Y., Nonlinear interactions of electromagnetic waves with the auroral ionosphere, AIP Conference Proceedings, 485, 18 (1999).

Wong, K.L., and Ono, M., Parametric decay of lower-hybrid waves in the ACT-1 toroidal device, Phys. Rev. Lett., 47, 842 (1981). 


\section{Chapter 7: Electrostatic and Electromagnetic Fluctuation Measurements}

There are many unanswered questions concerning density production, ion heating, and damping of the helicon wave in helicon sources. In Chapter 5, experimental evidence consistent with ion heating due to damping of slow waves downstream of the antenna was presented. However, the issues of ion heating near the antenna and the relationship between density production and the lower hybrid frequency were not addressed. The correlation between electron density and the on-axis lower hybrid frequency in helicon sources has been demonstrated in previous HELIX experiments [Balkey et al., 2001], by the data presented in Chapter 5 of this work, and by published data from other experiments [Boswell, 1984; Kwak et al., 1997; Yun et al., 1997; Kwak et al., 2000; Yun et al., 2001]. Yet in experiments at a single RF frequency, recent experiments using multiple ion species found no evidence of a correlation between density production and the lower hybrid frequency [Light, 2000]. Instead, Light [2000] suggests that the excitation of low frequency waves in helicon sources leads to anomalous particle transport and a constant or decreasing plasma density at high magnetic field strengths. The incompatibility of these observations illustrates the difficulty in developing a unified model of a plasma source for which a standard design or mode of operation is nonexistent.

In this chapter, experimental evidence of parametrically coupled electrostatic waves is presented for HELIX operating in the eight-coil configuration. The characteristics of the coupled electrostatic waves, e.g., wave number, dependence on RF power, radial variations, and dependence on RF frequency and magnetic field strength, are investigated. The electrostatic fluctuation measurements were made $35 \mathrm{~cm}$ from the end of the helicon antenna. Measurements of the electromagnetic wave spectrum are also reviewed. The electromagnetic wave measurements confirm that, except for RF frequencies well below the on axis lower hybrid frequency, the parametrically driven waves in HELIX are purely electrostatic. The electromagnetic wave measurements also confirm the $m=+1$ nature of the helicon waves propagating in the source. The magnetic 
fluctuation measurements were made at the same location as the electrostatic fluctuation measurements.

Parametrically driven waves and instabilities could provide a explanation for both the centrally peaked ion heating near the RF antenna and the correlation of plasma density and lower hybrid frequency. Since the plasma is generated with a large amplitude RF signal, the parametric excitation of waves in helicon sources should come as no surprise.

\subsection{Evidence of Parametrically Driven Electrostatic Waves}

The standard criteria for establishing that a set of waves is parametrically coupled are the two matching conditions, energy conservation and momentum conservation, described in the previous chapter: $\omega_{\mathrm{o}}=\omega_{1} \pm \omega_{2}$ and $k_{\mathrm{o}}=k_{1} \pm k_{2}$. A typical frequency spectrum from one tip of the electrostatic probe described in Chapter 3 is shown in Figure 72 for a HELIX plasma at a magnetic field strength of $840 \mathrm{G}$ and an RF frequency of 11 $\mathrm{MHz}$. Note that there is a clear electrostatic signature at the pump frequency. Tests confirm that the electrostatic signature of the pump wave is not due to inductive pickup from the RF antenna. The distinctly different electrostatic and electromagnetic fluctuation spectra (to be shown later) provide additional evidence that the observed electrostatic fluctuation spectra are not noise or RF pickup artifacts. The pump wave, $f_{o}=11 \mathrm{MHz}$, decays into a low frequency wave at $f_{1}=0.5 \mathrm{MHz}$ and side bands at $f_{o} \pm f_{1}(10.5$ and 11.5 $\mathrm{MHz}$ ). The low frequency side band (LSB) has more wave power than the upper side band (USB). Because the LSB wave requires less energy than the USB, $h\left(\omega_{o}-\omega_{1}\right)<h\left(\omega_{o}\right.$ $\left.+\omega_{1}\right)$ where $h$ is Planck's constant, a larger amplitude for the LSB than the USB is an expected feature of a parametric decay spectrum. The frequency spectrum shown in Figure 72 includes an additional electrostatic wave around $f \sim 9 \mathrm{MHz}$. The wave at 9 $\mathrm{MHz}$ does not change frequency with either magnetic field strength or RF driving frequency. The characteristics of this $9 \mathrm{MHz}$ feature will be reviewed later in this chapter. 


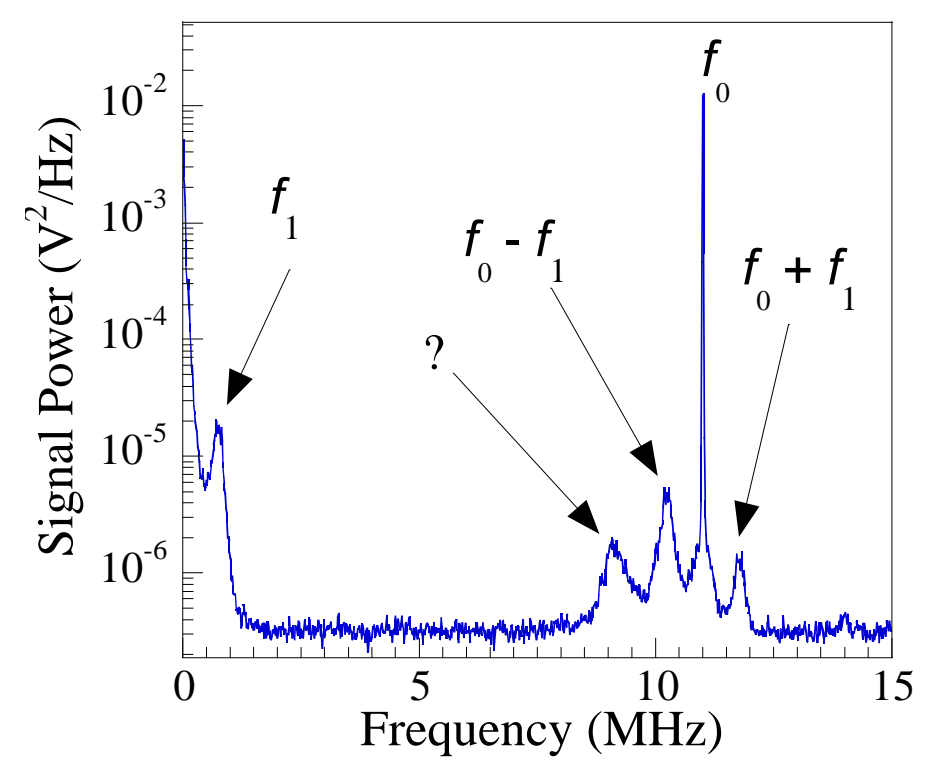

Figure 72: Parametric decay spectrum for an RF frequency of $11 \mathrm{MHz}$ and a magnetic field strength of 845 Gauss.

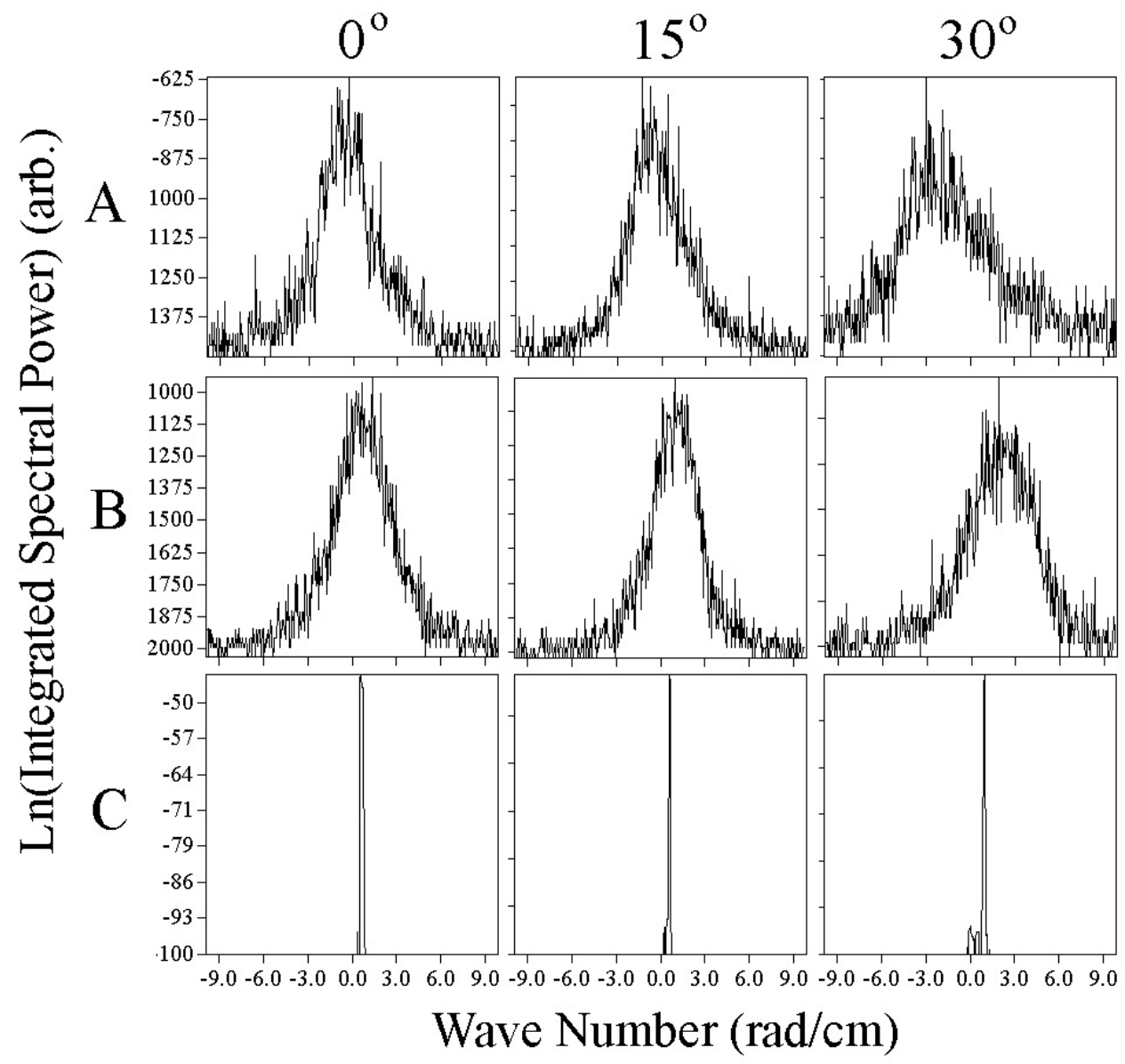

Figure 73: Natural logarithm of the spectral power integrated over $70 \mathrm{kHz}$ around the central wave frequency versus wave number for the (a) low frequency wave, (b) lower side band, and (c) pump wave at three different angles of the electrostatic probe tips relative to the axial magnetic field. 
The wave numbers for the low frequency wave, the LSB, and the pump wave shown in Figure 72 were measured using both tips of the electrostatic probe for three different angles with respect to the background magnetic field (Figure 73). Multiple measurement angles were used so that both the parallel and azimuthal wave numbers could be determined. The low frequency wave and the lower side band wave propagate in opposite directions (a positive wave number corresponds to propagation away from the antenna) while the pump wave has a small positive wave number. At all three measurement angles, the sum of the wave numbers for all three waves is zero to within the experimental error. For example, at $30^{\circ}$ the wave numbers are $k_{l f} \sim-3.0 \mathrm{rad} / \mathrm{cm}$ for the lower frequency component, $k_{l s b} \sim 2.4 \mathrm{rad} / \mathrm{cm}$ for the lower side band, and $k_{p} \sim 0.8$ $\mathrm{rad} / \mathrm{cm}$ for the pump. These wave number measurements demonstrate that the two sufficient conditions for parametric coupling, energy and momentum conservation, are met by electrostatic waves in HELIX.

\subsection{Characteristics of the Parametrically Coupled Electrostatic Waves in HELIX}

The wide range of operating frequencies, magnetic field strengths, and RF powers of HELIX provide a means of characterizing and identifying the parametrically coupled, electrostatic waves. In particular, as discussed in Chapter 6, parametrically driven instabilities should have a clearly defined threshold power for excitation.

\subsubsection{Variation of Parametrically Coupled Electrostatic Waves with RF Power}

The transfer of energy from the pump wave to the parametrically excited waves depends on the coupling strength (coupling coefficients) between the waves and the modulation amplitude (pump amplitude). Since the coupling strengths are constant for a given set of modes, the pump wave amplitude must exceed a threshold before parametric excitation can occur. Figure 74 shows the electrostatic power spectrum at four different $\mathrm{RF}$ input powers for a RF frequency of $11 \mathrm{MHz}$ and a magnetic field strength of $737 \mathrm{G}$. The power in the sidebands and the low frequency wave increases with increasing RF power. For these plasma parameters, parametric excitation occurs even for the lowest input power, 450 Watts, shown in Figure 74. For input powers less than 450 Watts, 
parametrically driven waves were not observed. However, at input powers less than 450 Watts HELIX drops out of the helicon mode into the inductive mode [Ellingboe and Boswell, 1996]. It appears that for these operating conditions the pump power required to operate the helicon source in the helicon mode exceeds the threshold for parametrically driving the additional electrostatic waves. Figure 75 shows the peak spectral power in the RF pump and lower side band waves as a function of RF input power. As expected for a parametrically driven wave, the peak power of the sideband wave increases linearly with increasing input power. The linear increase of the pump wave power with RF power provides a check of linearity of the probe response.

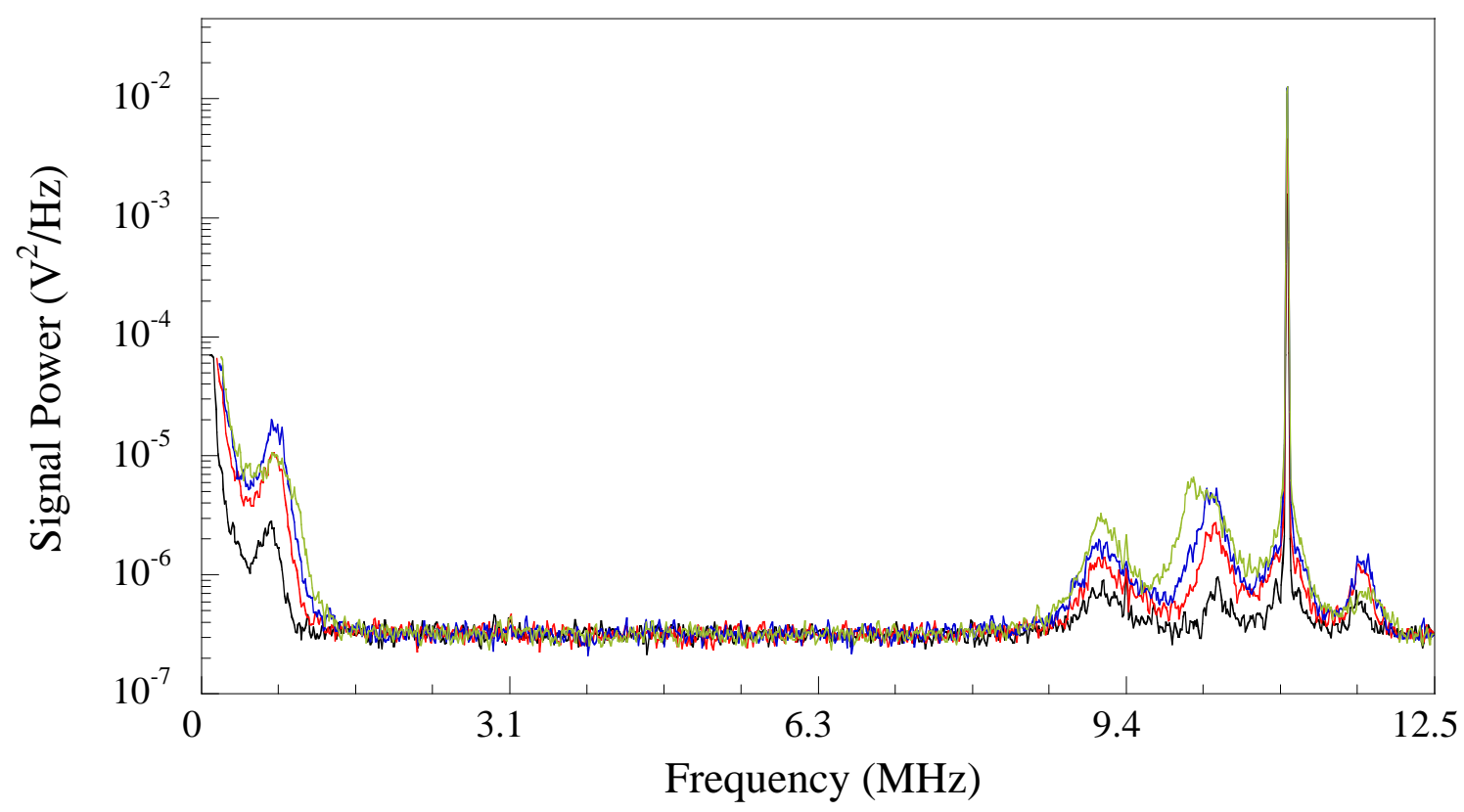

Figure 74: Electrostatic frequency spectra for a magnetic field strength of 845 Gauss, a RF frequency of 11 $\mathrm{MHz}$, and four different RF input powers: (-) 450 Watts, (-) 550 Watts, (-) 650Watts, and (-) 750 Watts. 


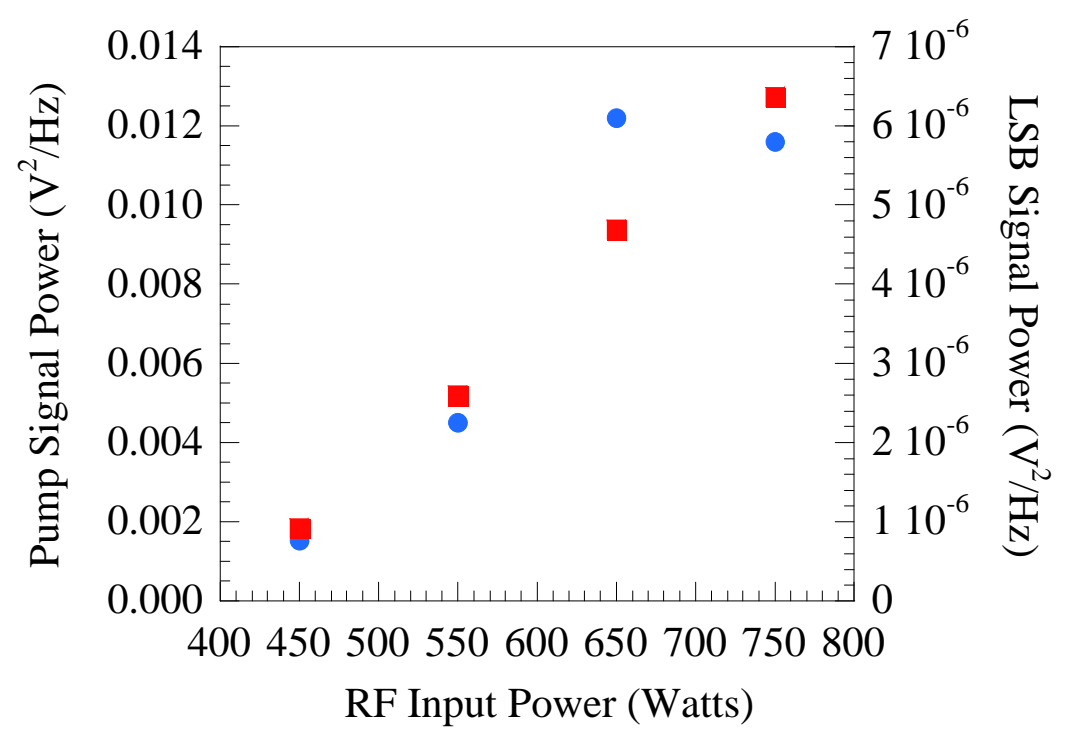

Figure 75: Peak spectral power of the (๑) pump and the ( $)$ lower side band waves versus RF power.

\subsubsection{Variation of Parametrically Coupled Electrostatic Waves with Radius}

To investigate the spatial dependence of the parametrically coupled waves, electrostatic spectra were measured at different radii for different RF frequencies and magnetic field strengths. Figure 76 shows typical electrostatic frequency spectra at different radial locations in the plasma. Spectra exhibiting parametrically coupled waves (a pump wave, upper and lower side bands, and a low frequency beat wave) occur only at radii less than $3 \mathrm{~cm}$. The reader is reminded that the feature at $9 \mathrm{MHz}$ is not evidence of parametric coupling. At radii beyond $3 \mathrm{~cm}$, there appears to be a low frequency wave, but it is not parametrically coupled to the pump wave because no side bands appear near the pump frequency. Therefore, the region of parametric coupling appears localized to the central, high density, region of the plasma.

Radial variations in electrostatic frequency spectra were also measured at different magnetic field strengths and driving frequencies. The peak spectral power in the pump, low frequency component, lower side band and upper side band waves for three different $\mathrm{RF}$ frequencies $(9,11$, and $13 \mathrm{MHz})$ and a magnetic field strength of 910 Gauss are shown in Figure 77 as a function of radial location. For all three RF frequencies, the parametrically coupled waves are localized to the center of the helicon source. The RF frequency is slightly below the on-axis lower hybrid frequency for the $9 \mathrm{MHz}$ case, 
slightly above for the $11 \mathrm{MHz}$ case, and well above for the $13 \mathrm{MHz}$ case. In all three cases, the electrostatic signature of the pump wave is largest on axis (Figure 77a). However, the on axis power for the $13 \mathrm{MHz}$ case is less than half the power observed for the two lower frequencies. At this magnetic field strength, higher plasma densities were obtained at 9 and $11 \mathrm{MHz}$ than at $13 \mathrm{MHz}$.

At $r=1 \mathrm{~cm}$, the power in at the pump frequency drops sharply for all three frequencies examined. At the same $r=1 \mathrm{~cm}$ radial location, the spectral power in the upper side band increases sharply for both the 9 and $11 \mathrm{MHz}$ cases (Figure 77c). Note that for the $13 \mathrm{MHz}$ case, very little lower side band power is measured at all radii. Across the entire inner region of the plasma, only the $9 \mathrm{MHz}$ case exhibits a significant level of upper side band power (Figure 77d). The $9 \mathrm{MHz}$ case corresponds to the conditions at which maximum ion heating was observed near the RF antenna (see Chapter 5). For all three frequencies, the spectral power in the low frequency wave peaked in the inner region of the plasma (Figure 77b). The on axis power of the low frequency mode was largest for the $11 \mathrm{MHz}$ case. For the two other magnetic field strengths investigated, 640 and 1185 Gauss, the amplitudes of the parametrically coupled waves were largest on axis and followed the same general trends as shown in Figure 77.

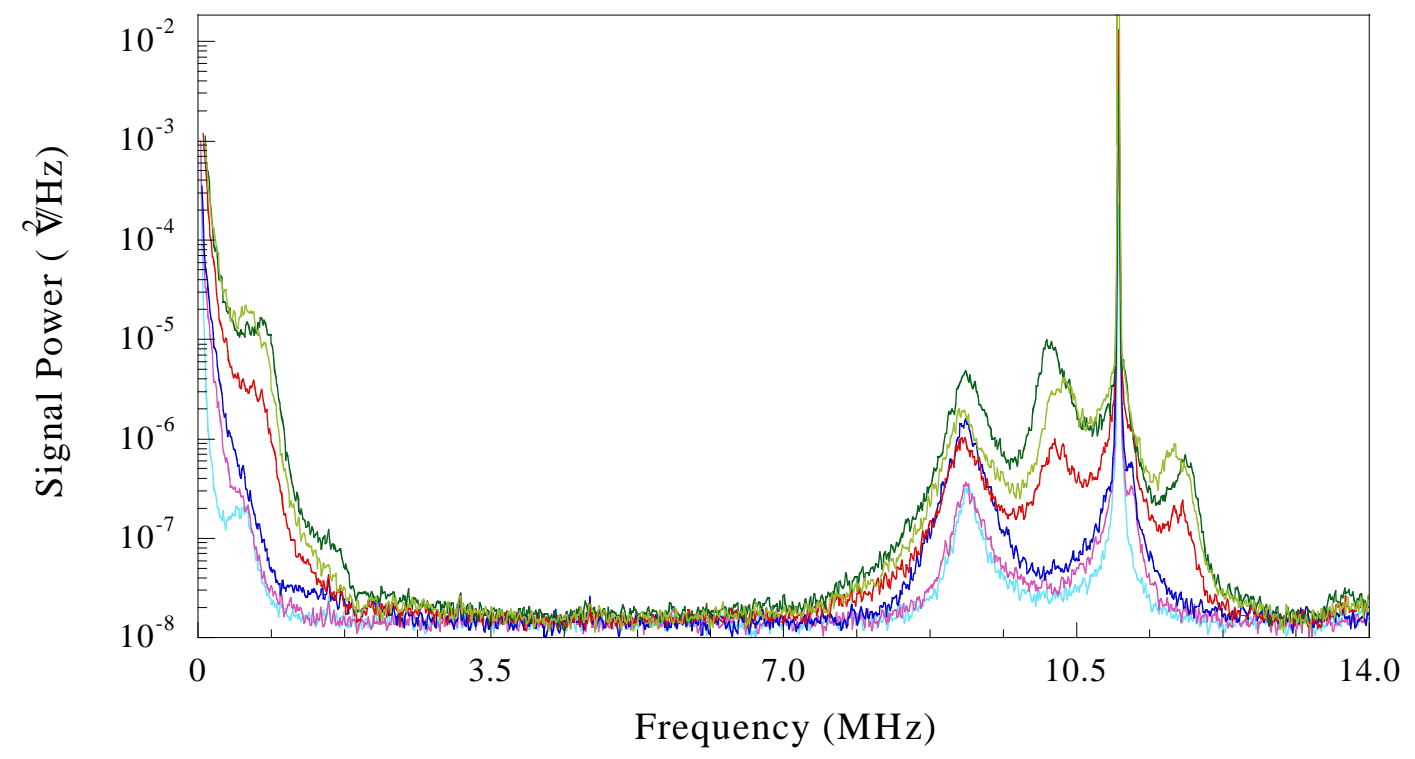

Figure 76: Electrostatic spectra for a magnetic field strength of $910 \mathrm{G}$, a RF frequency of $11 \mathrm{MHz}$, and six different plasma radii (-) $r=5 \mathrm{~cm},(-) r=4 \mathrm{~cm},(-) r=3 \mathrm{~cm},(-) r=2 \mathrm{~cm},(-) r=1 \mathrm{~cm}$, and $(-) r=$ $0 \mathrm{~cm}$. 

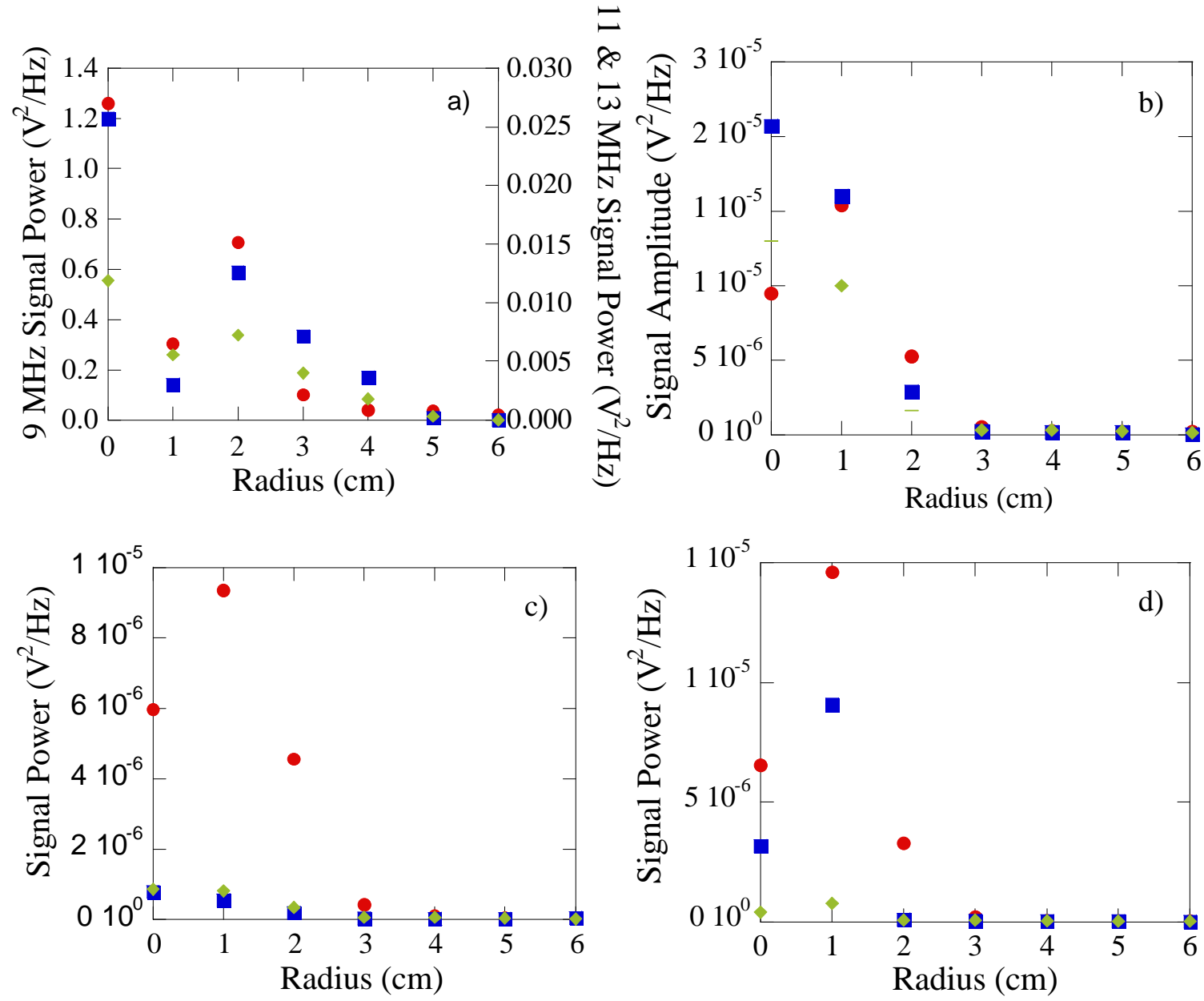

Figure 77: Peak spectral power at a magnetic field strength of $910 \mathrm{G}$ versus radius for RF frequencies of $(\bullet) 9 \mathrm{MHz},(\boldsymbol{\square}) 11 \mathrm{MHz}$, and $(\diamond) 13 \mathrm{MHz}$ for the a) pump b) low frequency c) lower side band and d) upper side band waves.

\subsubsection{Variation of Parametrically Coupled Electrostatic Waves with RF Frequency and Magnetic Field Strength}

As shown in Figure 77, the relative amplitudes of the parametrically coupled electrostatic waves are a strong function of the RF frequency of the pump wave. As was done for the ion temperatures presented in Chapter 5, the amplitudes of the various electrostatic waves were measured as a function of both RF frequency and magnetic field strength. Because the plots of electrostatic wave amplitude versus magnetic field strength and RF frequency are quite complicated, it is instructive to begin with comparisons of 
electrostatic spectra at different RF frequencies but at the same magnetic field strength. Electrostatic spectra for a magnetic field strength of 845 Gauss and an RF power of 750 Watts are shown in Figure 78 for seven different RF frequencies.
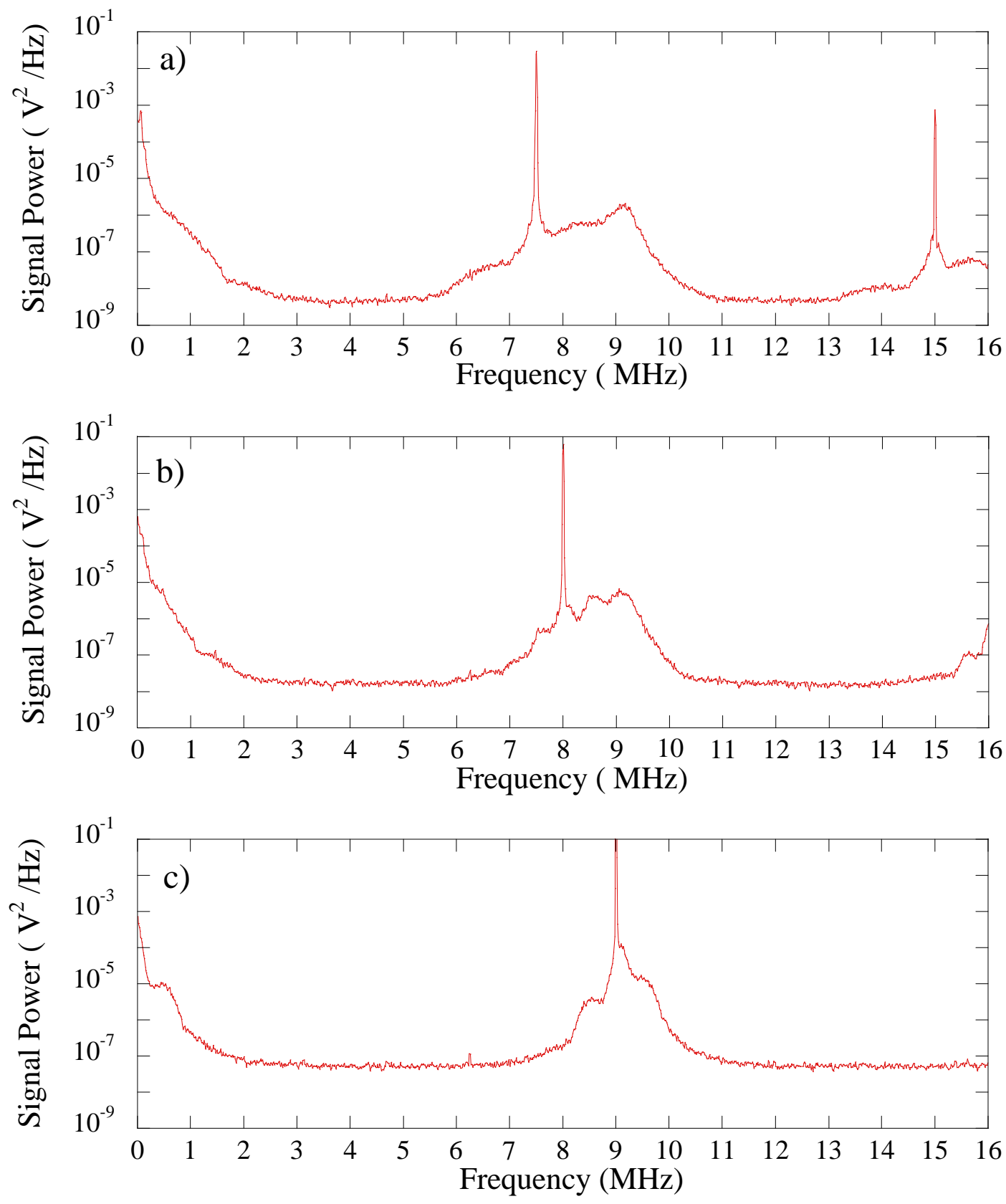

Figure 78: Electrostatic spectra for a magnetic field strength of 845 Gauss, a RF power of 750 Watts and for seven different RF frequencies. Shown here are a) $7.5 \mathrm{MHz}$, b) $8 \mathrm{MHz}$, and c) $9 \mathrm{MHz}$. 

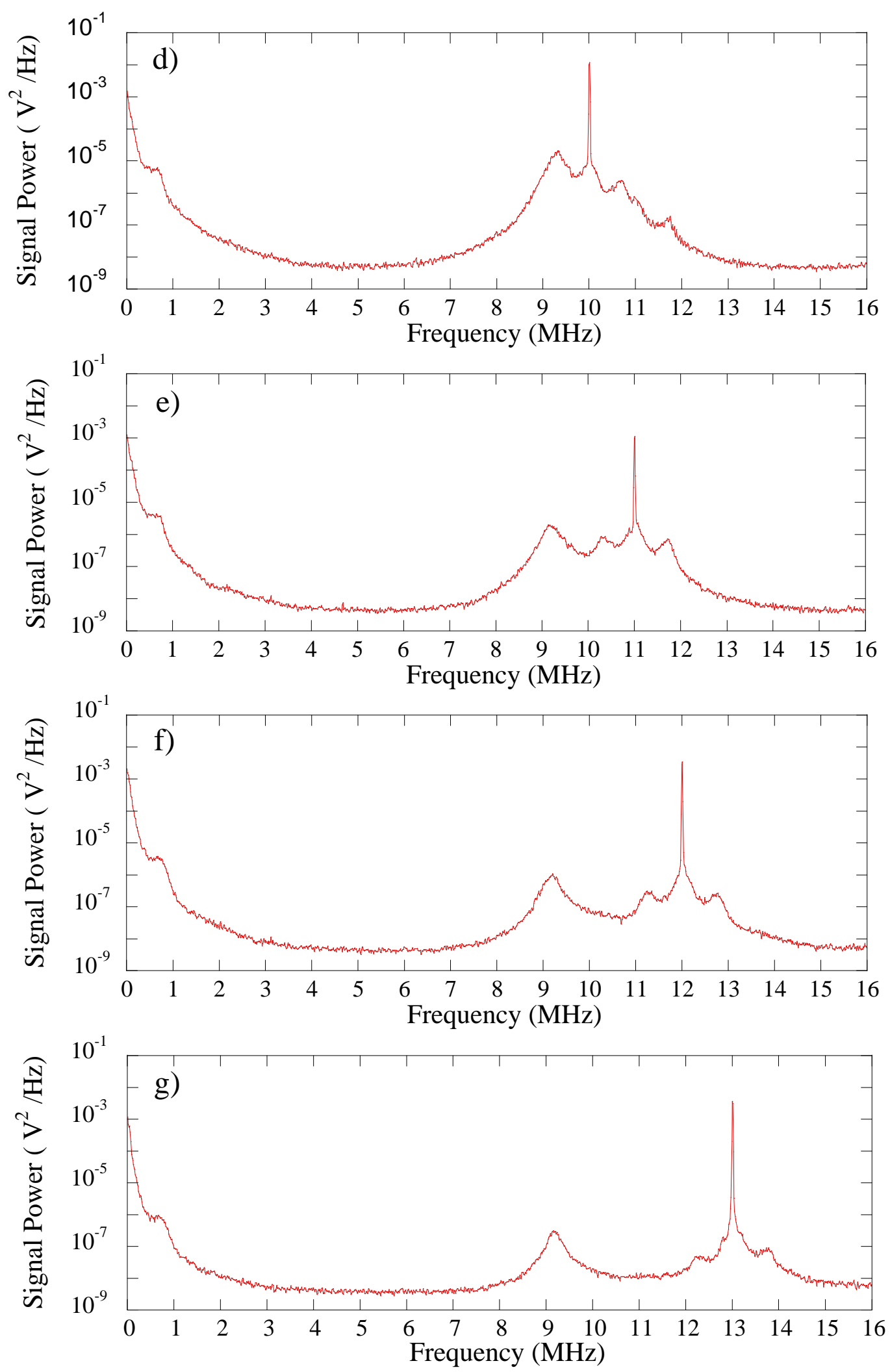

Figure 78: Electrostatic spectra for a magnetic field strength of 845 Gauss, a RF power of 750 Watts and for seven different RF frequencies. Shown here are d) $10 \mathrm{MHz}$, e) $11 \mathrm{MHz}$, f) $12 \mathrm{MHz}$, and g) $13 \mathrm{MHz}$. 
Keeping in mind that the $9 \mathrm{MHz}$ wave seen in every plot in Figure 78 is not parametrically coupled to the pump wave, the data shown in Figure 78 indicate that at low frequencies, 7.5 and $8 \mathrm{MHz}$, the upper side band wave contains more power than the lower side band wave. At higher frequencies, 10 and $11 \mathrm{MHz}$, the lower side band wave has more power than the upper side band wave. As mentioned previously, the lower frequency sidebands in a typical parametric decay spectrum have larger amplitudes than the higher frequency sidebands. These measurements suggest that the coupling coefficients may favor a parametric excitation of waves near the on-axis lower hybrid frequency, i.e., resonant coupling. For a magnetic field of 845 Gauss, the on-axis, lower hybrid frequency is approximately $8.7 \mathrm{MHz}$. For RF frequencies of 7.5 and $8 \mathrm{MHz}$, the lower hybrid frequency is above the RF frequency. For RF frequencies of 10 and 11 $\mathrm{MHz}$, the lower hybrid frequency is below the RF frequency. The change in relative amplitudes of upper and lower side bands could also reflect a change in the damping of those modes. In all cases, however, the energy and momentum conservation relations are still satisfied for the sidebands, the pump wave, and the low frequency wave.

The power in each electrostatic wave component as a function of RF frequency and magnetic field strength is shown in Figure 79. Perhaps most surprising is the drop in the pump wave power for those RF frequencies for which the RF frequency is below the onaxis, lower hybrid frequency (Figure 79d). For the same parameters, there is a peak in the amplitude of the low frequency wave $(\sim 500 \mathrm{kHz})$. These measurements suggest damping of the pump wave by excitation of the low frequency beat wave. Details of the wave number and other characteristics of the low frequency wave will be presented in the next section of this chapter. The power in the low frequency wave is a maximum for RF frequencies well below the on-axis lower hybrid frequency. For these same plasma parameters, the perpendicular ion heating near the antenna was a maximum (see Figure 65 in Chapter 5).

The spectral power in the pump and lower side band waves peaks for an RF frequency of $10 \mathrm{MHz}$ and magnetic field strengths around 1000 Gauss. As shown in Figure 65 in Chapter 5, these are nearly the same conditions that yielded the largest downstream perpendicular ion temperatures. With regard to the 


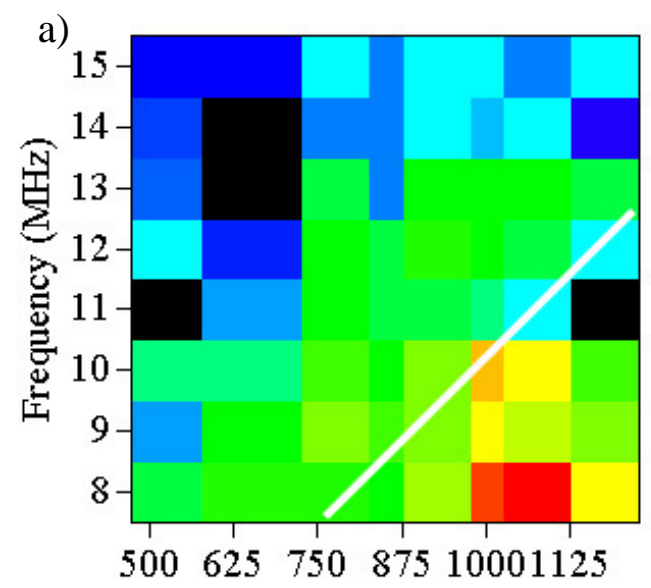

Magnetic Field Strength (Gauss)
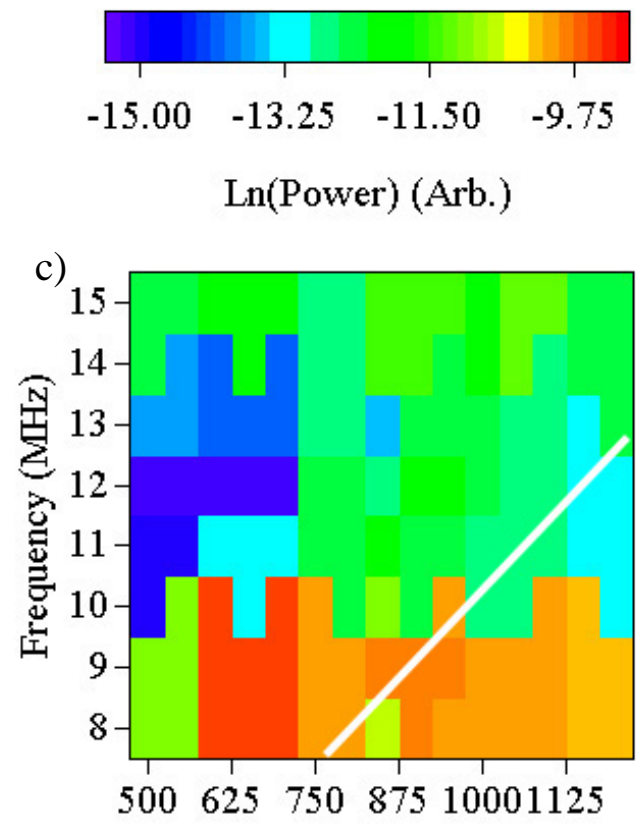

Magnetic Field Strength (Gauss)

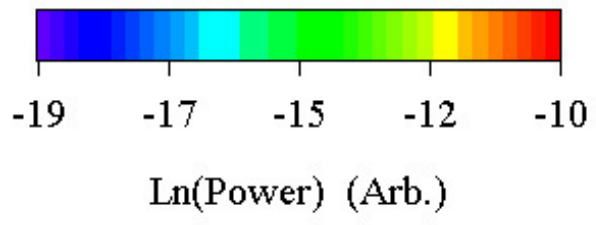

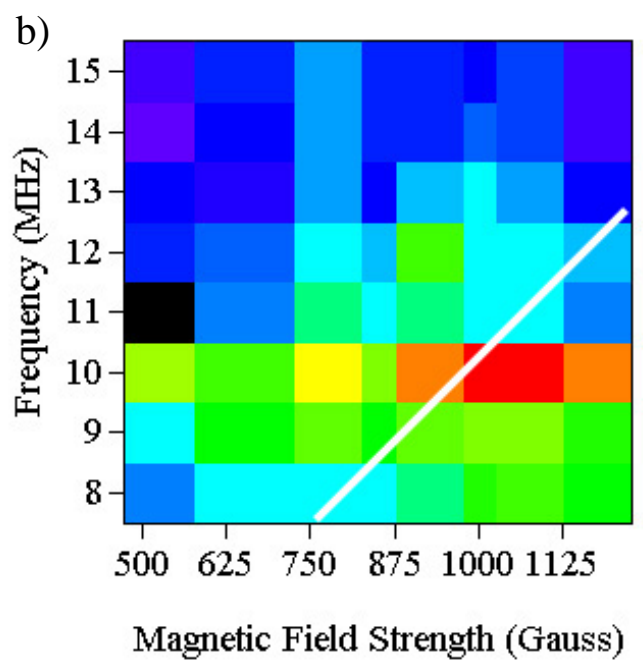

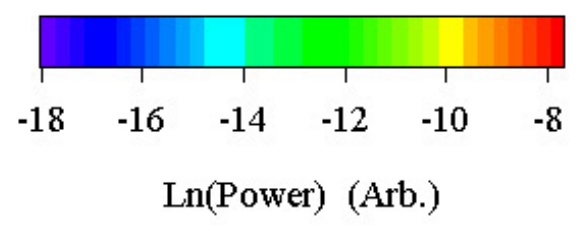

d)

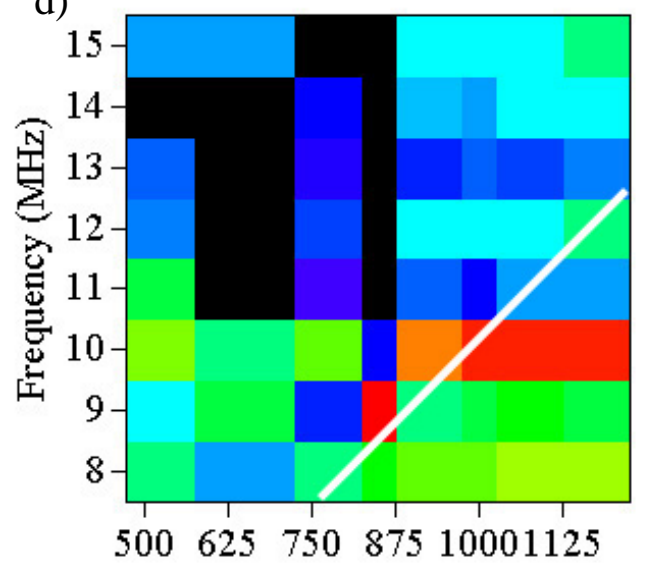

\section{Magnetic Field Strength (Gauss)}

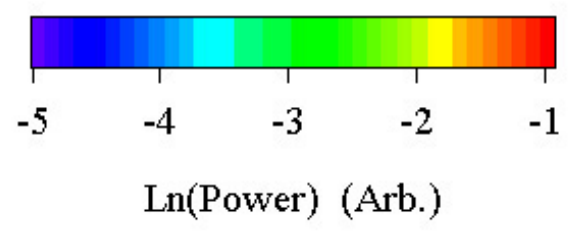

Figure 79: Peak electrostatic spectral power versus RF frequency and magnetic field strength of the a) low frequency, b) lower side band, c) upper side band and d) pump waves. 
perpendicular ion temperatures, the maximum ion temperatures occurred at an RF frequency of $9 \mathrm{MHz}$ and the same range of magnetic field strengths that the power in the electrostatic wave at pump frequency has a clear maximum at a particular value of magnetic field strength and RF frequency suggests that system dimensions and boundary conditions in the eight-coil configuration favor excitation of electrostatic waves around $10 \mathrm{MHz}$. At a magnetic field of roughly 1000 Gauss, the on-axis lower hybrid frequency is approximately $10 \mathrm{MHz}$. Thus, the electrostatic waves at the pump frequency and the lower side band are most strongly driven when the RF frequency matches the lower hybrid frequency. Electrostatic waves propagating at or very near to the lower hybrid frequency are typically called electrostatic lower hybrid waves [Stix, 1965; Swanson, 1989]. A key signature of electrostatic lower hybrid waves is backwards propagation, i.e., group and phase velocities that are opposite in sign [Swanson, 1989]. The power in the upper side band wave is greatest for the lowest RF frequencies examined and peaks at a magnetic field strength of approximately 650 Gauss. There appears to be little correlation with power in the upper side band and either plasma density production or ion heating in HELIX.

\subsubsection{Wave Number Measurements for the Parametrically Coupled Electrostatic Waves}

To understand how the parametrically coupled electrostatic waves could couple energy into the ions or electrons in HELIX, the waves must be identified. In a plasma, waves are best identified by their dispersion relation. By measuring the wave numbers of the pump, side band, and low frequency waves as a function of RF driving frequency, the dispersion relation for each of the waves can be obtained and used to identify the wave.

The parallel and azimuthal wave numbers verses RF frequency for the pump, upper side band, and low frequency waves at a radius of $2 \mathrm{~cm}$ and a magnetic field strength of 845 Gauss are shown in Figure 80. Except at 10 and $11 \mathrm{MHz}$, the parallel wave numbers for the pump wave are on the order of $k_{\|} \sim .4 \mathrm{rad} / \mathrm{cm}$, i.e., parallel wavelengths of approximately $16 \mathrm{~cm}$. The most obvious choice to excite an approximately $16 \mathrm{~cm}$ long electrostatic wave at the RF frequency is the $19 \mathrm{~cm}$ long RF antenna. Since the parallel 
wave numbers do not change significantly with RF frequency, the pump wave has a zero parallel group velocity, i.e., it is an externally imposed perturbation and not a naturally propagating electrostatic plasma wave. At 10 and $11 \mathrm{MHz}$, the parallel wavelength of the pump wave suddenly decreases to approximately 6 and $3 \mathrm{~cm}$, respectively. Since such short parallel wavelengths are clearly not externally imposed, the measurements indicate a fundamental change in the nature of the pump wave at these two frequencies. The finite parallel group velocity suggested by the frequency dependence of the wave numbers suggests the excitation of a convective instability in the plasma. With only two data points, determination of the dispersion relation was not attempted. It is worth noting that the maximum plasma density production was observed for similar RF frequencies (10 and $11 \mathrm{MHz}$ ) and magnetic field strengths (see Chapter 5) and this issue will be revisited later in this dissertation. The azimuthal wave numbers correspond to an $m=+1$ wave, $k_{\theta}$ $\sim 0.26$ or $\lambda \sim 24 \mathrm{~cm}$.

The azimuthal wave numbers for the upper side band wave (Figure 80b) start at $k_{\theta}=-7.5 \mathrm{rad} / \mathrm{cm}$ and decrease (in magnitude) to about $k_{\theta}=-5.0 \mathrm{rad} / \mathrm{cm}$ at an $\mathrm{RF}$ frequency of $9 \mathrm{MHz}$. Above $9 \mathrm{MHz}$ there is almost no change in the perpendicular wave number with RF frequency. As noted previously in Figure 78 and Figure 79, the power in the upper side band wave is largest at low frequencies, below $9 \mathrm{MHz}$, for a magnetic field strength of 845 Gauss. For 845 Gauss, the on axis lower hybrid frequency is $f_{l h} \sim 8.7$ $\mathrm{MHz}$ and therefore the frequency of the upper side band wave is close to the lower hybrid frequency until the RF frequency exceeds $9 \mathrm{MHz}$. The azimuthal wave number data between 7.5 and $9.0 \mathrm{MHz}$ have the unique signature of a backward propagating wave. Keeping in mind that the frequency of the upper side band wave for these plasma parameters was always a constant 400 to $800 \mathrm{kHz}$ above the RF frequency of the pump wave, the phase velocity, $\omega / k_{\theta}$, of the upper side band wave is negative while the group velocity, $d \omega / d k_{\theta}$ is positive, i.e. backwards propagation. Backward propagation is characteristic of electrostatic lower hybrid waves [Swanson, 1989]. Thus, for RF frequencies below $9 \mathrm{MHz}$, the upper side band wave can be identified as an electrostatic lower hybrid wave. Similar measurements of the lower side band indicate that it too is an electrostatic lower hybrid wave, but only when the RF frequency is above $9 \mathrm{MHz}$ for a magnetic field strength of 845 Gauss. 

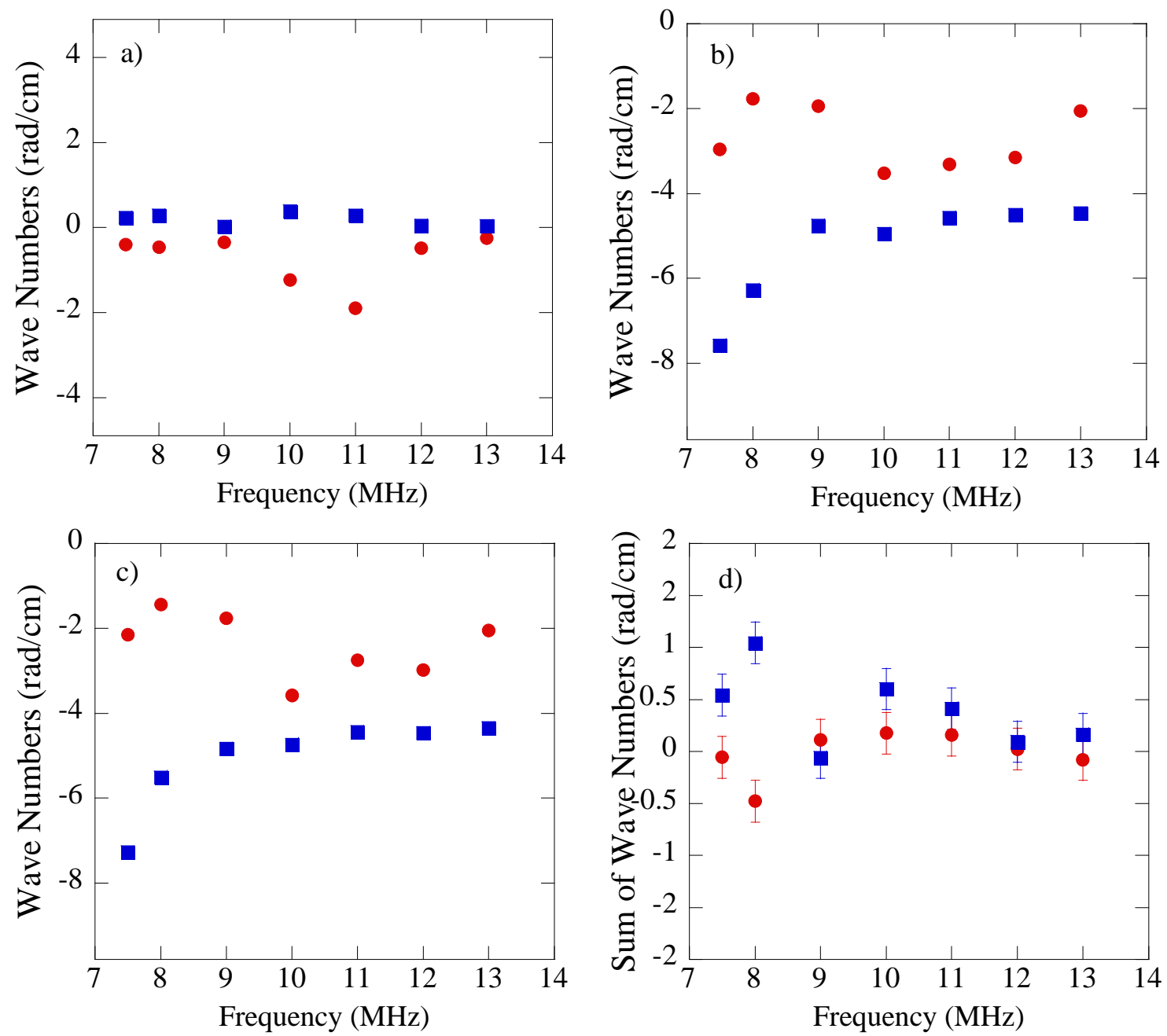

Figure 80: ( ) Azimuthal and (๑) parallel wave numbers for the a) pump, b) upper side band, c) low frequency waves versus RF frequency for a magnetic field strength of 845 Gauss. The wave number sum, $k_{r f}+k_{u s b}-k_{l f}$, versus RF frequency is shown in d). For comparison, all wave numbers are plotted verses the RF driving frequency and not the individual wave frequencies. The side bands were always a constant 400 to $800 \mathrm{kHz}$ away from the pump frequency and the low frequency wave was always in the range of 400 to $800 \mathrm{kHz}$.

Both the parallel and azimuthal wave numbers for the low frequency wave track the upper side band wave numbers as a function of RF frequency (Figure 80b and Figure 80c). The near one-to-one correspondence between the low frequency wave numbers and upper side band wave numbers is due to the momentum conservation parametric coupling condition and the small wave number magnitudes of the pump wave. The wave number sum, $k_{r f}+k_{u s b}-k_{l f}$, is plotted in Figure $80 \mathrm{~d}$ as a function of RF frequency (not the actual wave frequencies). The perpendicular wave numbers are conserved for all frequencies 
except at $8 \mathrm{MHz}$ and the parallel wave numbers are conserved for frequencies above 8 MHz. In general, the measured wave numbers are in agreement with the wave number matching condition.

For RF frequencies above $9 \mathrm{MHz}$, both the frequency and azimuthal wave number of the low frequency wave remained constant, $f \sim 500 \mathrm{kHz}$ and $k_{\theta} \sim-4.4 \mathrm{rad} / \mathrm{cm}$ (Figure 80c). If the low frequency wave is an azimuthally propagating ion acoustic wave and therefore the measured wave phase speed $(\omega / k)$ equals the ion sound speed, the wave measurements correspond to electron temperatures of approximately $10 \mathrm{eV}$. For RF frequencies below $9 \mathrm{MHz}$, the frequency and azimuthal wave number of the low frequency wave increase. The electron temperatures calculated from the measured wave phase speeds, assuming the low frequency wave is an ion acoustic wave, are shown in Figure 81 along with the electron temperatures measured on axis with the Langmuir probe as a function of RF frequency. Although higher than electron temperatures typical of other helicon source experiments, the plotted values are comparable to the Langmuir probe measurements of electron temperature.

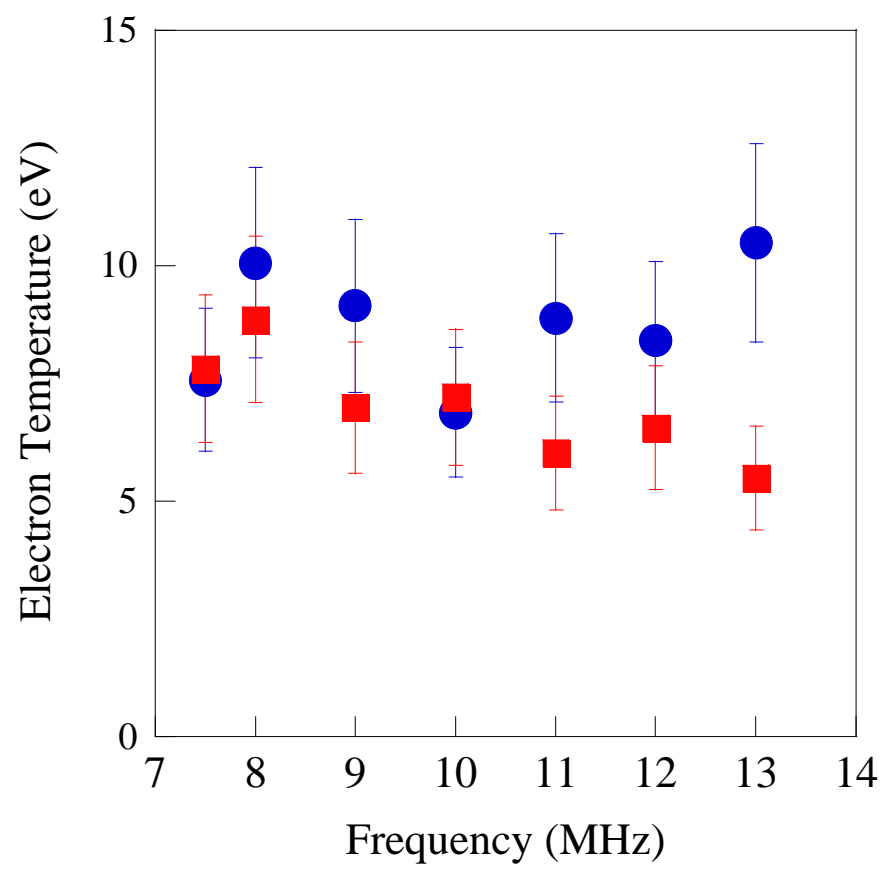

Figure 81: Electron temperature measured by the ( $\square)$ Langmuir probe and calculated for an (๑) ion acoustic wave using the measured wave frequency and wave number with $20 \%$ error bars. 


\subsection{Characteristics of Electromagnetic Waves in HELIX}

Although the wavelength and spatial structure of the electromagnetic, "helicon," wave has been examined by other groups as a function of RF frequency [Shoji et al., 1993], magnetic field strength [Jiwari et al., 1994; Chen et al., 1995; Sakawa et al., 1999], and density [Shinohara, 1997], Balkey's electromagnetic fluctuation measurements in the allglass version of HELIX were the first detailed, simultaneous measurements of the electromagnetic frequency and wave number spectra in a helicon source [Balkey, 2000]. Balkey's measurements were limited to high frequencies (above $500 \mathrm{kHz}$ ), had poor azimuthal spatial resolution, and were accomplished outside of the glass vacuum chamber [Balkey, 2000]. Using the electromagnetic fluctuation probe described in Chapter 3, the wave numbers and fluctuation spectra for electromagnetic waves in HELIX were measured as a function of RF frequency and magnetic field strength.

\subsubsection{Wave Number Measurements for the Primary Electromagnetic Wave}

The measured wave number of the primary RF as a function of probe angle relative is shown in Figure 82. The measurements were made at a radius of $2 \mathrm{~cm}$ for a magnetic field strength of 845 Gauss and an RF frequency of $9 \mathrm{MHz}$. The wave numbers smoothly vary from $k_{\|}=0.20 \mathrm{rad} / \mathrm{cm}$ parallel to the magnetic field ( $0^{\circ}$ probe angle) to $k_{\theta}=0.60$ $\mathrm{rad} / \mathrm{cm}$ in the azimuthal direction ( $90^{\circ}$ probe angle). The radial wave number, $k_{r}$, is fixed by the plasma chamber boundary [Blevin and Christiansen, 1966; Boswell, 1984] or at the plasma radius where the plasma density becomes too low to support a helicon wave [Keiter et al., 1997]. The perpendicular wave number is related to $k_{\mathrm{r}}$ and $k_{\theta}$ by $k_{\perp}=\left(k_{r}^{2}+\right.$ $\left.k_{\theta}^{2}\right)^{1 / 2}$; however, $k_{\mathrm{r}}$ was not measured in these experiments The corresponding wavelengths are $\lambda_{\|}=32 \mathrm{~cm}$ and $\lambda_{\theta}=10.5 \mathrm{~cm}$ respectively. If the wavelength of the helicon wave is imposed by the half wavelength, $19 \mathrm{~cm}$ long, RF antenna, the parallel wave number would be $k_{\|} \sim 0.17 \mathrm{rad} / \mathrm{cm}$, consistent with the experimentally measured value. At a radius of $\sim 2 \mathrm{~cm}$, an $m=+1$ azimuthal mode would have a wave number of $k_{\theta} \sim 0.50 \mathrm{rad} / \mathrm{cm}$, consistent with the experimentally measured value. Assuming the radial boundary condition determines the radial wave number, $k_{\mathrm{r}} \sim(2 \pi / 5 \mathrm{~cm})=1.3$ 
$\mathrm{rad} / \mathrm{cm}$ and therefore $k_{\perp} \sim 1.4 \mathrm{rad} / \mathrm{cm}$. Thus, the electromagnetic wave appears to be an $m=+1$ wave at a parallel wavelength equal to twice the antenna length.

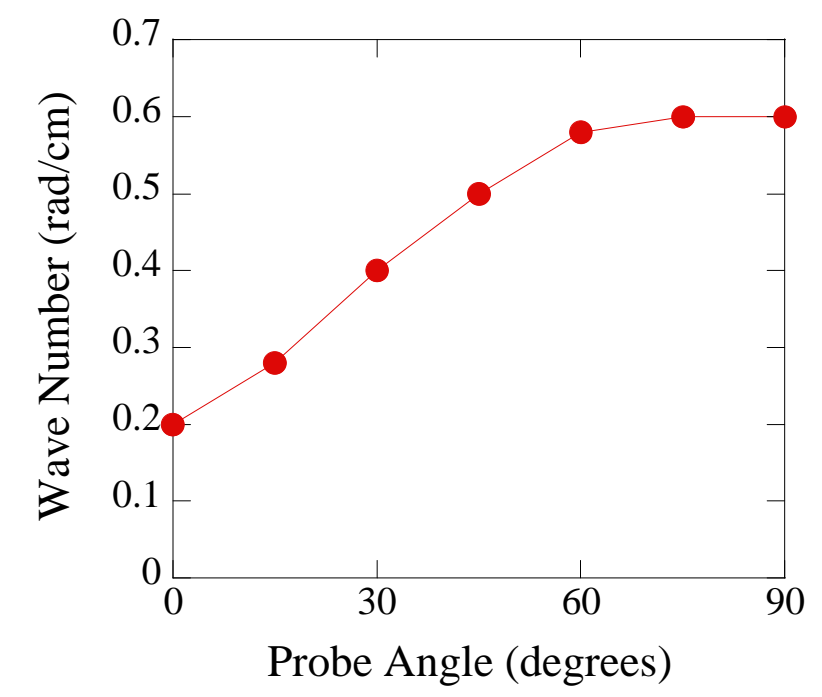

Figure 82: Measured wave number at the RF frequency versus angle of the magnetic probe relative to the background magnetic field direction. The magnetic field strength was 845 Gauss and the RF frequency was $9 \mathrm{MHz}$.

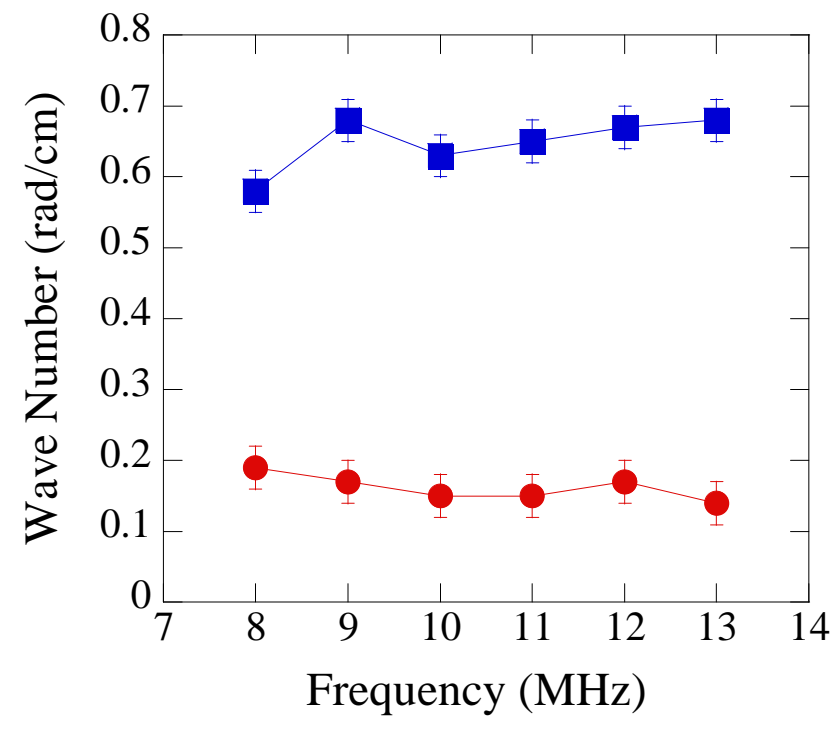

Figure 83: (-) Azimuthal and (๑) parallel wave numbers for the primary RF wave versus RF frequency at a magnetic field strength of 845 Gauss and a radial location of $2 \mathrm{~cm}$.

The azimuthal and parallel wave numbers for a magnetic field strength of 845 Gauss are plotted versus RF frequency in Figure 83. The parallel wave numbers decrease 
slightly with increasing RF frequency and the azimuthal wave numbers increase slightly with increasing RF frequency. Assuming the RF wave satisfies the simple helicon wave dispersion relation, the data of Figure 83 could also be interpreted as a measurement of density versus RF frequency. The simple helicon dispersion relationship can be written as

$$
n=\left(5.1 \times 10^{14}\right) k_{\|} \mid f \mathrm{~cm}^{-3},
$$

where $f$ is in MHz, $n$ is the density in units of $\mathrm{cm}^{-3}$, the magnetic field is 845 Gauss, and the small aspect ratio approximation $\left(k_{\perp}>k_{\|}\right)$[Chen, 1991; Keiter et al., 1997] has been used to calculate the perpendicular wave number. With these assumptions, the parallel wave numbers shown in Figure 83 correspond to a plasma density of $1.2 \times 10^{13} \mathrm{~cm}^{-3}$ at an RF frequency of $8 \mathrm{MHz}$ that decreases to $5 \times 10^{12} \mathrm{~cm}^{-3}$ at an RF frequency of $13 \mathrm{MHz}$, consistent with the measured decrease in plasma density with increasing RF frequency (see Chapter 5 and the measurements reported by Balkey [Balkey et al., 2001]). These measurements demonstrate that the primary RF wave satisfies the helicon wave dispersion relation. Previous measurements have shown that the wave number of the primary RF wave satisfies the helicon dispersion relation [Boswell, 1984; Chen et al., 1995], but these are the first such measurements as a function of RF frequency.

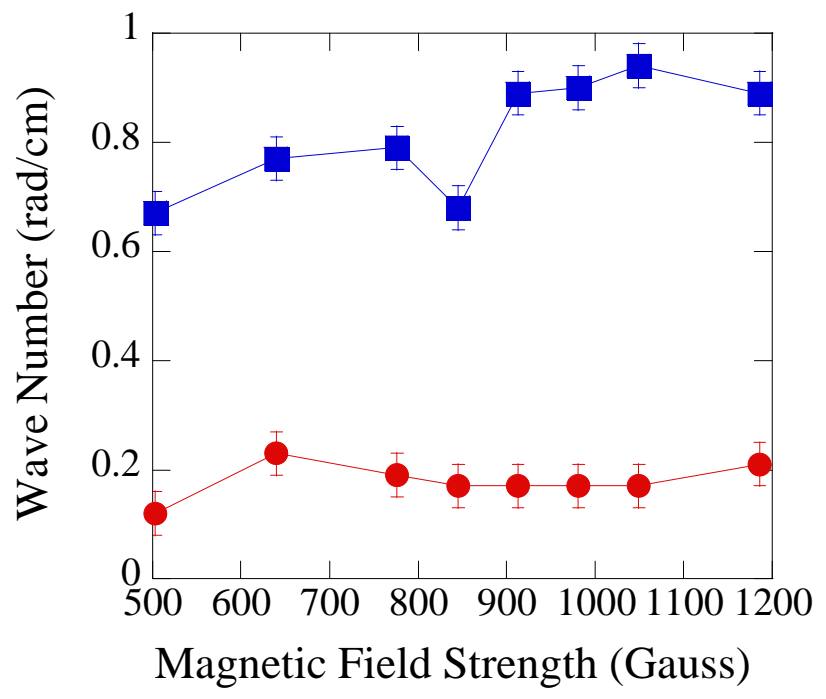

Figure 84: ( ) Azimuthal and ( $\bullet$ parallel wave numbers versus magnetic field strength at an RF frequency of $9 \mathrm{MHz}$ and a radial location of $2 \mathrm{~cm}$. 
In Figure 84, the azimuthal and parallel wave numbers are plotted as a function of magnetic field strength at $r=2 \mathrm{~cm}$ for an RF frequency of $9 \mathrm{MHz}$. As the magnetic field increases, around 850 Gauss the azimuthal mode makes a transitions from an $m \sim 1$ mode $\left(k_{\theta} \sim 0.50 \mathrm{rad} / \mathrm{cm}\right)$ to shorter wavelengths corresponding to an $m \sim 2$ mode $\left(k_{\theta} \sim 1.0\right.$ $\mathrm{rad} / \mathrm{cm})$. A similar transition in the azimuthal mode number at the edge of the plasma for magnetic field strengths around 850 Gauss was seen in previous HELIX experiments [Balkey, 2000]. Concomitant with the increase in azimuthal mode number, Balkey also observed a sharp increase in the parallel wave number [Balkey, 2000]. No transition in parallel wave number is evident in these internal measurements.

The parallel wave numbers are relatively constant above 700 Gauss and correspond to a parallel wavelength of roughly $35 \mathrm{~cm}$, about twice the antenna length. Rewriting the simple helicon dispersion relationship as a function of magnetic field,

$$
n=\left(6.2 \times 10^{13}\right) k_{\|} B \mathrm{~cm}^{-3},
$$

where $B$ is the magnetic field in kilogauss, $n$ is the density in units of $\mathrm{cm}^{-3}$, the wave frequency is $9 \mathrm{MHz}$, and the small aspect ratio approximation $\left(k_{\perp}>k_{\|}\right)$is made [Chen, 1991; Keiter et al., 1997]. Therefore, if the simple helicon wave dispersion relation is satisfied, the parallel wave number data of Figure 84 suggest that the plasma density increases with increasing magnetic field at an RF frequency of $9 \mathrm{MHz}$. At $1000 \mathrm{G}$, Equation (7.2) predicts a plasma density of $1.1 \times 10^{13} \mathrm{~cm}^{-3}$, consistent with previous experimental measurements at similar source parameters. If the details of actual perpendicular wave number were included in Equation (7.2), the jump in azimuthal mode number at higher magnetic fields would result in larger predicted plasma densities.

\subsubsection{Electromagnetic Fluctuation Spectra}

To determine if the parametrically coupled electrostatic waves were electromagnetic or purely electrostatic, the frequency spectrum of electromagnetic fluctuations was also measured. Figure 85 shows the electromagnetic spectrum for six different RF driving frequencies. The plotted frequency spectra were not corrected for frequency dependent coil area effects or intrinsic resonances in the coils. The numbers 1 and 2 mark the locations of the two strongest coil resonances (see Chapter 3, Figure 29b). Spectral features occurring at coil resonances are noise artifacts and should be ignored. 
The only spectrum that exhibits any features typical of parametric coupling is the 8 $\mathrm{MHz}$ case. Above $8 \mathrm{MHz}$, only the externally driven RF wave is observed. Therefore, above $9 \mathrm{MHz}$ and for a magnetic field of 845 Gauss, all of the side band and low frequency waves observed with the electrostatic probe are purely electrostatic. Although both probes detect waves at the RF frequency, the significantly different parallel wavelengths measured by the two probes suggest that both an electromagnetic and an electrostatic wave are being excited at the RF frequency. The $8 \mathrm{MHz}$ spectrum includes parametric-like side bands and a low frequency beat wave. The electromagnetic side bands of the pump wave are closer to the pump frequency, $\Delta f \sim 200 \mathrm{kHz}$, than the electrostatic side bands, $\Delta f \sim 600 \mathrm{kHz}$ (Figure 86). Note that the $8 \mathrm{MHz}$ case is the only one for which the RF driving frequency is below the lower hybrid frequency; the same conditions at which strong perpendicular ion heating attributed to damping of the slow wave was observed downstream of the antenna.

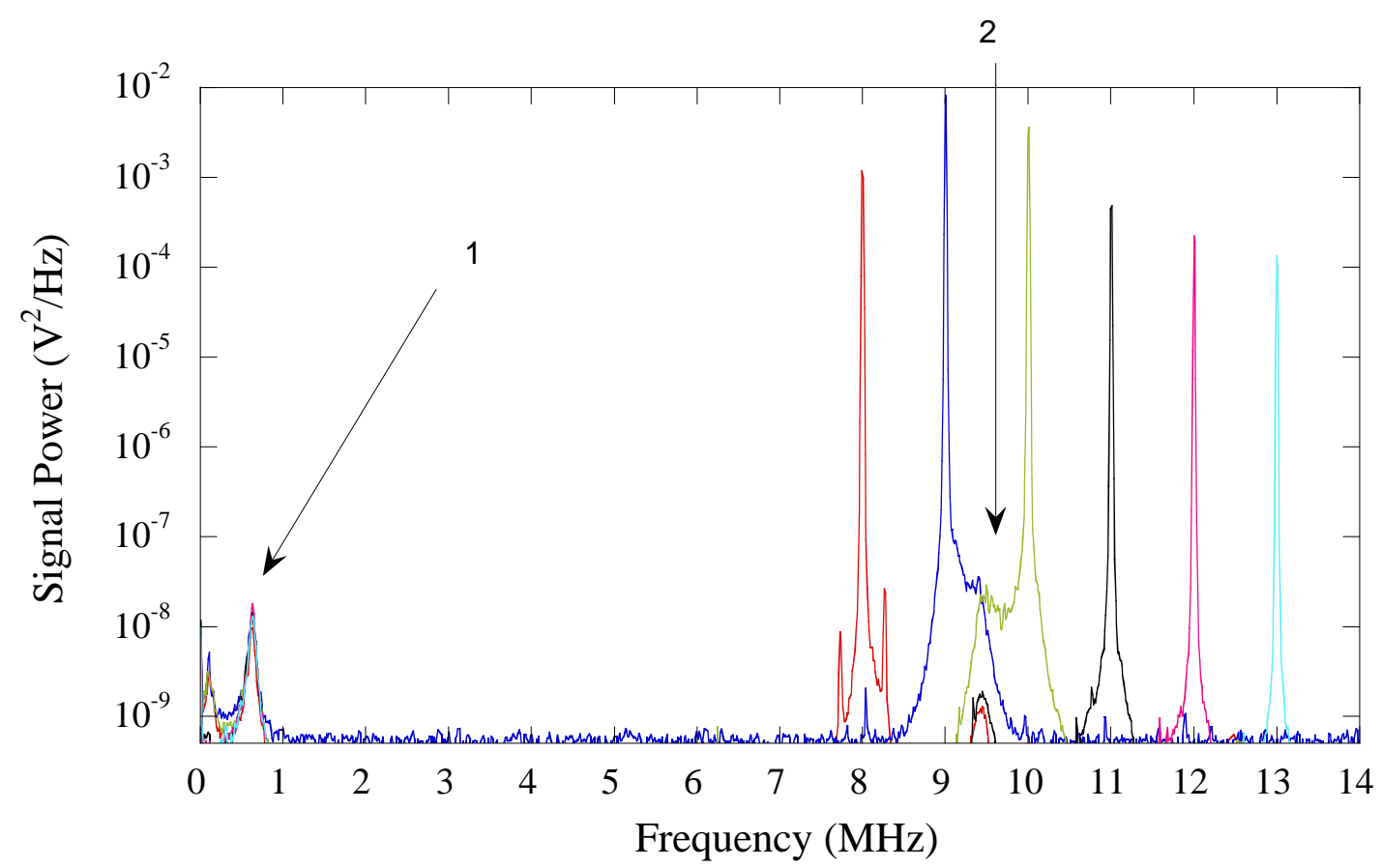

Figure 85: Frequency spectra of electromagnetic fluctuations for a magnetic field strength of 845 Gauss and for six different RF frequencies: (-) $f=8 \mathrm{MHz}$. (-) $f=9 \mathrm{MHz}$. (-) $f=10 \mathrm{MHz}$. (一) $f=11 \mathrm{MHz}$, $(-) f=12 \mathrm{MHz}$, and $(-) f=13 \mathrm{MHz}$. The numbers 1 and 2 indicate the location of intrinsic resonances in the sense coils and peaks at those frequencies are spurious. 


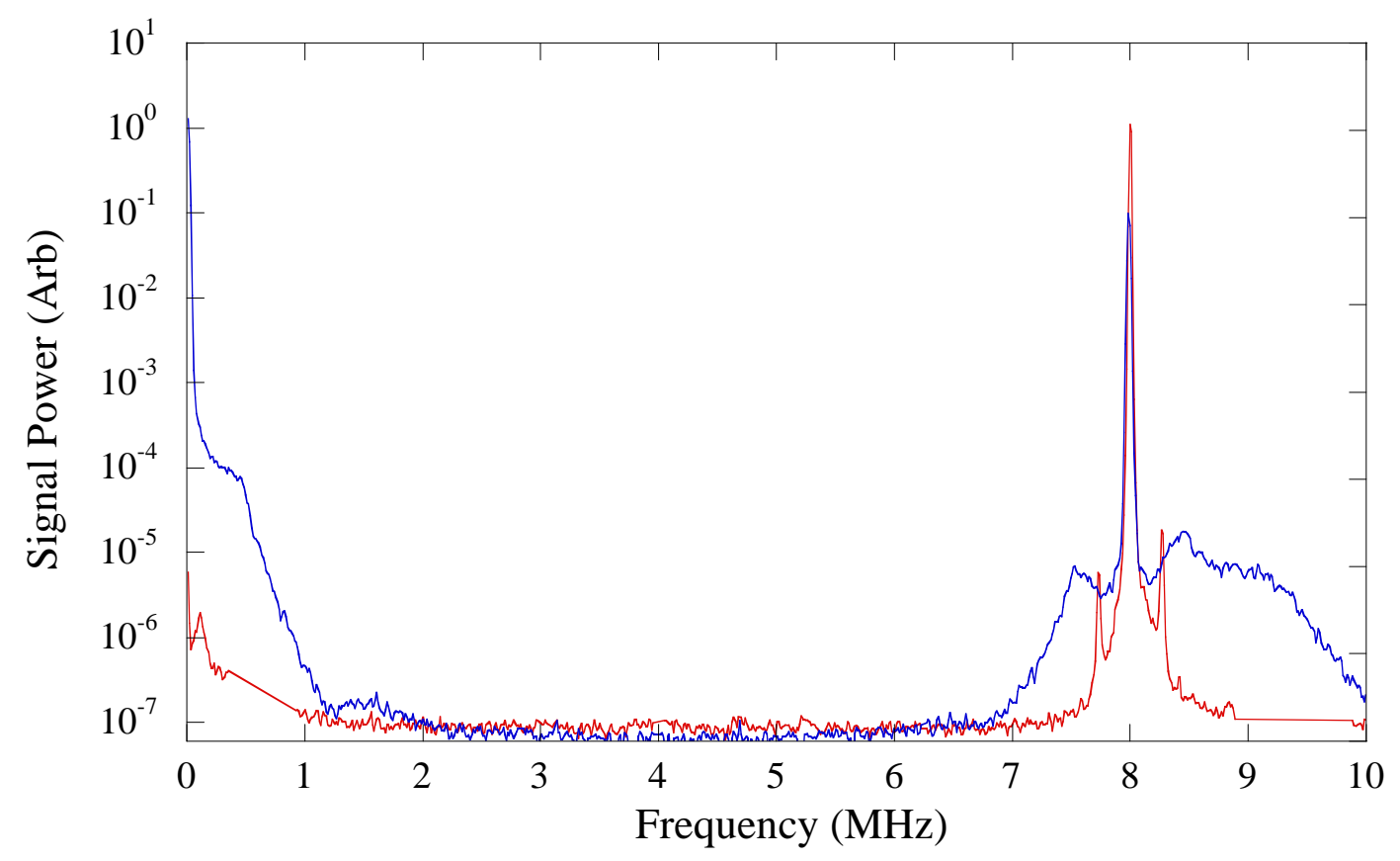

Figure 86: (-) Electromagnetic frequency spectrum and (-) electrostatic frequency spectrum at an RF frequency of $8 \mathrm{MHz}$ and a magnetic field strength of 845 Gauss. The electromagnetic spectrum has been modified to eliminate the spurious peaks at the sense coil resonance frequencies.

\subsection{Implications of the Electrostatic and Electromagnetic Fluctuation Measurements}

The data presented here demonstrate that parametrically coupled electrostatic waves exist in helicon sources and could play important roles in both plasma density production and perpendicular ion heating. Parametrically driven waves in helicon sources are not surprising given the number of previous experiments that have observed parametrically driven waves when similar plasmas are driven with comparable strength RF waves [Wong and Ono, 1981]. The electrostatic probe measurements indicate that the power in the parametrically driven waves is largest near the axis of the helicon source. The only electromagnetic wave that can propagate in this region of the helicon source is the helicon wave since the slow or "Trivelpiece-Gould" wave is strongly damped at the plasma edge [Chen and Arnush, 1997]. However, the significantly different parallel wave numbers measured by the electrostatic and electromagnetic probes suggests that a shorter wavelength electrostatic wave may also be excited on axis by the RF antenna. For RF frequencies just above the on-axis lower hybrid frequency, the parallel 
wavelength of the electrostatic wave at the RF frequency drops sharply while the parallel wavelength of the electromagnetic wave is unchanged. This behavior suggests that only the electrostatic wave experiences a resonance at the lower hybrid frequency. Akhiezer [1998] has suggested that damping of the helicon wave by the excitation of parametrically driven instabilities explains the measured helicon wave damping rates [Chen, 1996; Chen et al., 1996]. In that model, short wavelength ion sound turbulence is most efficiently driven for RF frequencies near the lower hybrid frequency. The ion sound turbulence scatters electrons, leading to electron heating and increased density production. Such a mechanism would explain the correlation between the lower hybrid frequency and plasma density production in HELIX and would be consistent with a peaked density profile. The predicted perpendicular wavelengths for the ion sound turbulence are too small to be measured with probes. However, recent microwave scattering experiments indicate very short wavelength, ion acoustic-like turbulence is generated in helicon sources [Kaganskaya et al., 2001].

Not only can parametric coupling of the helicon wave to electrostatic waves be responsible for the observed helicon wave damping rates, the parametrically driven side band and low frequency waves can also provide a mechanism to couple RF power directly into the plasma particles. To couple wave energy directly into the electrons, the phase velocity of the wave must be near the electron thermal velocity. For the frequency range of $8-11 \mathrm{MHz}$, the parallel phase speed, $\omega / k_{\|}$, of the electrostatic side band waves is on the order of $2.7 \times 10^{7}-3.8 \times 10^{7} \mathrm{~cm} / \mathrm{s}$, or $0.4-0.8 \mathrm{v}_{\text {the }}$. Given that it is possible for such waves to Landau damp on the electrons, it should be possible to see evidence of such interactions in the electron temperature data. The power in the lower side band and the measured electron temperature in the eight-coil configuration are shown in Figure 87 as a function of magnetic field strength and RF frequency. As mentioned previously, the extremely high electron temperatures are actually due to what appears to be a drift in the electron distribution. Nevertheless, there is a clear correlation between increased power in the lower side band wave and increased "energy" in the electrons.

To couple wave energy into the ions, the phase speed of the wave must be close to the ion thermal speed. For wave numbers less than $10 \mathrm{rad} / \mathrm{cm}$ (the maximum wave number resolvable with the electrostatic probe), the wave frequency must be below $900 \mathrm{kHz}$ to be 
less than five times the thermal speed of $0.5 \mathrm{eV}$ ions. Thus, only the low frequency electrostatic wave or the low frequency electromagnetic wave observed for RF frequencies below the lower hybrid frequency could directly couple energy into the ions. Figure 88 shows the perpendicular ion temperature $5 \mathrm{~cm}$ from the front of the antenna and the power in the low frequency electrostatic wave as a function of magnetic field strength and RF driving frequency. At high magnetic field strengths, above 900 Gauss, the peak in the power in the lower frequency electrostatic wave is well correlated with the peak in the perpendicular ion temperature. Both the perpendicular ion temperature and wave power plots also have a region of reduced intensity around an RF frequency of 11 $\mathrm{MHz}$ and a magnetic field strength of 1100 Gauss. These observations suggest that near the RF antenna, the parametrically driven low frequency electrostatic wave either directly heats the ions or is driven by the same mechanism responsible for the ion heating. A similar investigation of the low frequency electromagnetic wave was not performed but is clearly warranted based on these electrostatic wave measurements.

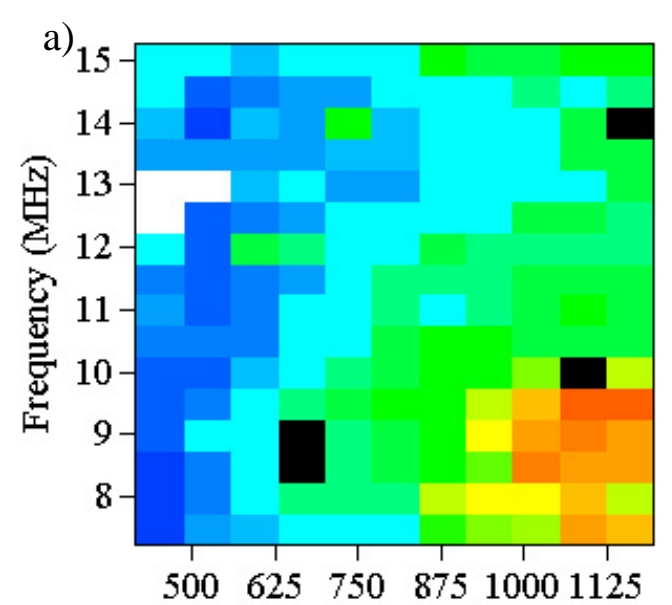

Magnetic Field Strength (Gauss)
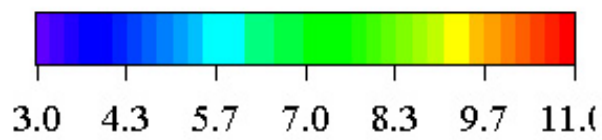

Electron Temperature (eV)

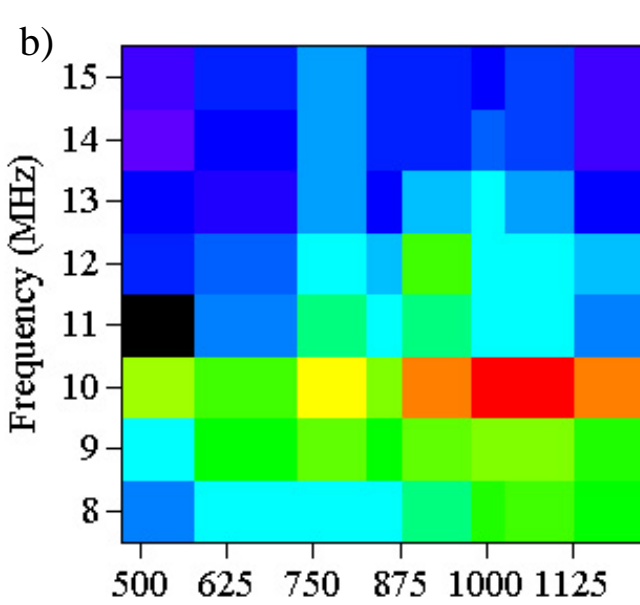

Magnetic Field Strength (Gauss)

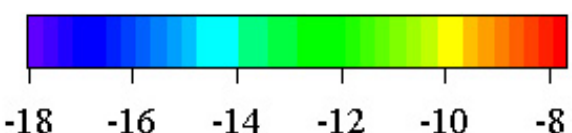

Ln(Power) (Arb.)

Figure 87: a) Electron temperature, b) power in lower side band wave as a function of magnetic field strength and RF driving frequency. 


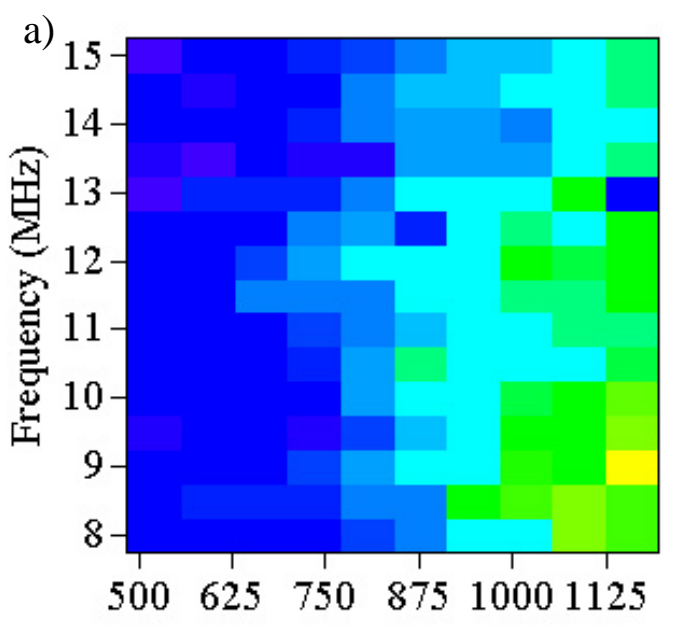

Magnetic Field Strength (Gauss)

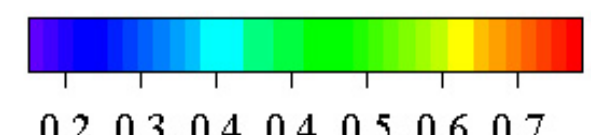

$\begin{array}{llllllll}0.2 & 0.3 & 0.4 & 0.4 & 0.5 & 0.6 & 0.7\end{array}$

Perpendicular Ion Temperature (eV)

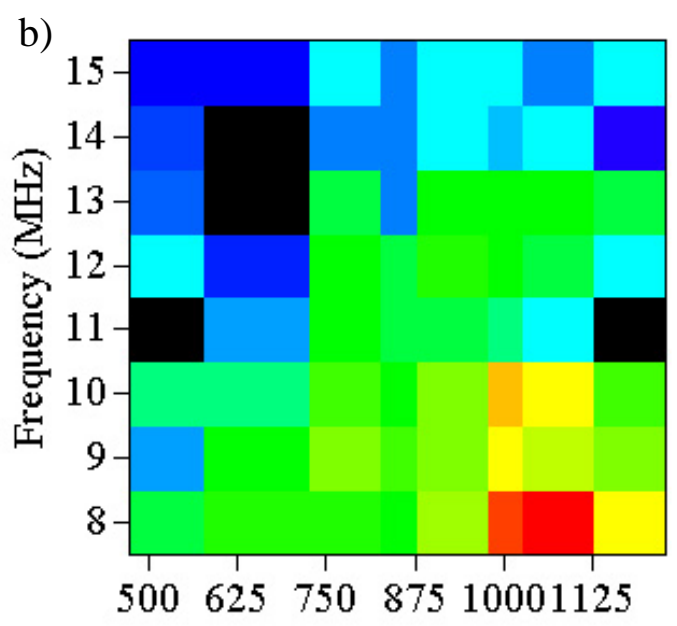

Magnetic Field Strength (Gauss)

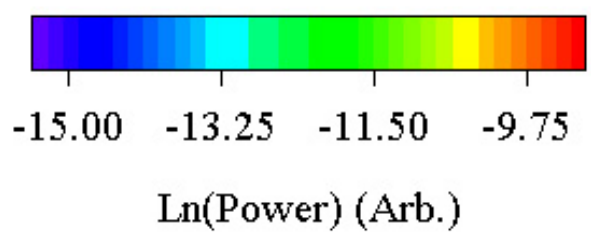

Figure 88: a) Perpendicular ion temperatures measured $5 \mathrm{~cm}$ in the front of the antenna and b) power in the low frequency electrostatic wave

\subsection{The Anomalous 9 MHz Wave}

Before concluding the discussion of the electrostatic and electromagnetic wave measurements, the anomalous wave at $9 \mathrm{MHz}$ must be addressed. As shown in Figure 89, the $9 \mathrm{MHz}$ wave observed in all of the electrostatic fluctuation measurements is purely electrostatic, i.e., there is not a similar wave in the electromagnetic fluctuation spectra (Figure 85). To check if the $9 \mathrm{MHz}$ was an instrumental artifact, electrostatic fluctuation spectra were obtained with two different digitizers and at two different digitization rates (Figure 89). Since the frequency spectra are the same in both cases, the $9 \mathrm{MHz}$ wave is not artificially created by temporal aliasing or internal noise in one of the digitizers. The $9 \mathrm{MHz}$ wave is also not caused by an ambient noise source in the laboratory environment. Figure 90 shows an electrostatic frequency spectrum obtained with all of the laboratory 
equipment turned on (including the RF amplifier) but without argon in the vacuum chamber. The frequency spectrum shows the expected $1 /$ frequency noise spectrum with two unidentified peaks at $\sim 150$ and $\sim 300 \mathrm{kHz}$, but no signal around $9 \mathrm{MHz}$. Phase difference measurements yield wave numbers on the order of $6 \mathrm{rad} / \mathrm{cm}$ for the $9 \mathrm{MHZ}$ wave in the perpendicular direction. The frequency of the wave changes by less than 100 $\mathrm{kHz}$ over the entire RF frequency and magnetic field strength operating range of HELIX and the wave amplitude does increase with increasing RF input power (Figure 74). All experimental tests indicate that the $9 \mathrm{MHz}$ wave is a real electrostatic wave. Because of the wave's fixed frequency, it appears to be a fundamental electrostatic mode of the plasma that is independent of magnetic field strength, RF frequency, and plasma density. At this time, no electrostatic wave meeting all of these constraints has been identified.
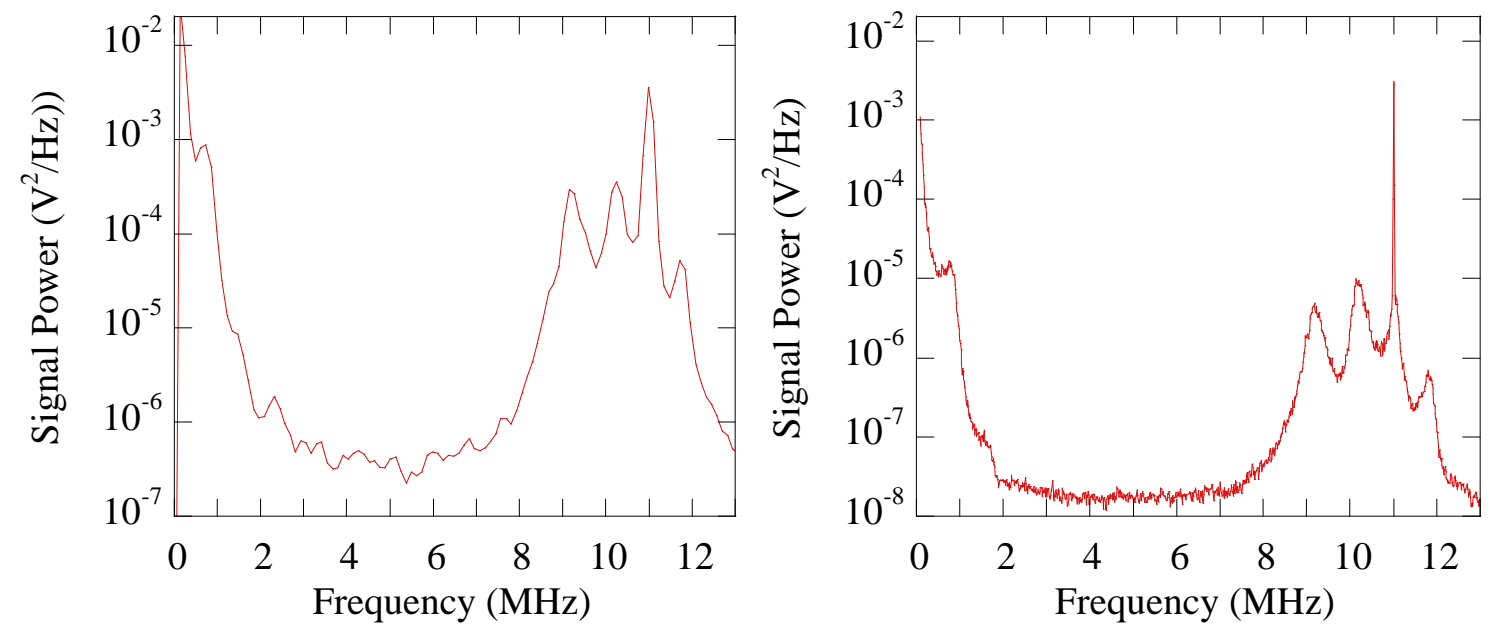

Figure 89: electrostatic frequency spectra showing the anomalous $9 \mathrm{MHz}$ wave obtained with two different instruments at two different digitization rates. a) Data from the TVS 641 digitizer at a sampling rate of 100 Gigasamples/s. b) Data from the TDS 430A oscilloscope at a sampling rate of 0.1 Gigasamples/s. 


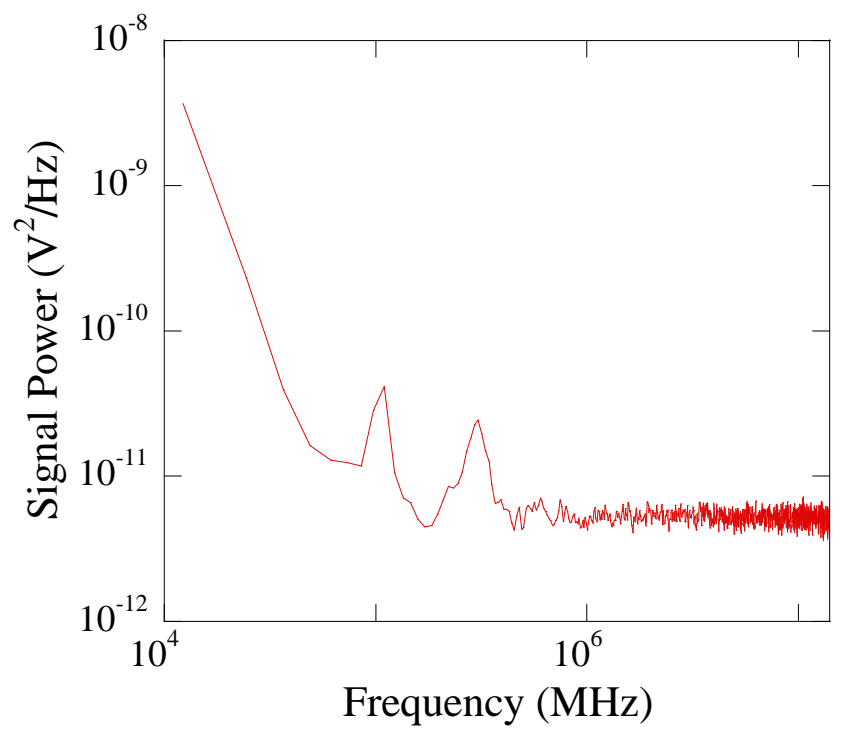

Figure 90: Electrostatic frequency spectrum obtained with all of the laboratory equipment turned on (including the RF amplifier) but without argon in the vacuum chamber. 


\section{Chapter 7 References}

Akhiezer, A.I., Mikhailenko, V.S., and Stepanov, K.N., Ion-sound parametric turbulence and anomalous electron heating with application to helicon plasma sources, Phys. Lett. A, 245, 117 (1998).

Balkey, M.M., Optimization of a Helicon Plasma Source for Maximum Density with Minimal Ion Heating, Ph. D. thesis, West Virginia University, Morgantown (2000).

Balkey, M.M., Boivin, R.F., Kline, J.L., and Scime, E.E., Ion heating and density production in helicon sources near the lower hybrid frequency, Plasma Sources Sci. Technol., 10, 284 (2001).

Blevin, H.A., and Christiansen, P.J., Austrailian Journal of Physics, 15, 501 (1966).

Boswell, R.W., Very efficient plasma generation by whistler waves near the lower hybrid frequency, Plasma Phys. Controlled Fusion, 26, 1147 (1984).

Chen, F.F., Plasma ionization by helicon waves, Plasma Phys. Controlled Fusion, 33, 339 (1991).

Chen, F.F., Physics of helicon discharges, Phys. Plasmas, 3, 1783 (1996).

Chen, F.F., and Arnush, D., Generalized theory of helicon waves. I. Normal modes, Phys. Plasmas, 4, 3411 (1997).

Chen, F.F., Sudit, I.D., and Light, M., Downstream physics of the helicon discharge, Plasma Sources Sci. Technol., 5, 173 (1996).

Chen, R.T.S., Breun, R.A., Gross, S., Hershkowitz, N., Hsieh, M.K., and Jacobs, J., Experimental studies of multimode helicon plasma waves, Plasma Sources Sci. Technol., 4, 337 (1995).

Ellingboe, A.R., and Boswell, R.W., Capacitive, inductive and helicon-wave modes of operation of a helicon plasma source, Phys. Plasmas, 3, 2797 (1996).

Jiwari, N., Fukasawa, T., Kawakami, H., Shindo, H., and Horiike, Y., Helicon wave plasma reactor employing single-loop antenna, J. Vac. Sci. Technol. A, 12, 1322 (1994).

Kaganskaya, N.M., Krämer, M., and Selenin, V.L., Enhanced-scattering experiments on a helicon discharge, Phys. Plasmas, 8, 4694 (2001).

Keiter, P.A., Scime, E.E., and Balkey, M.M., Frequency dependent effects in helicon plasmas, Phys. Plasmas, 4, 2741 (1997).

Kwak, J.G., Choi, H.D., Bak, H.I., Suwon, C., Bak, J.G., and Kim, S.K., Frequency dependence of the plasma density for helicon plasmas, Phys. Plasmas, 4, 1463 (1997).

Kwak, J.G., Kim, S.K., and Cho, S., Upper limit to the monotonic increasing dependence of the plasma density on the magnetic field in helicon discharges, Phys. Lett. A, 267, 384 (2000).

Light, M.E., A study of Low Frequency Instability and Transport in a Helicon Plasma, $\mathrm{Ph}$. D. thesis, University of California - Los Angeles, Los Angeles (2000).

Sakawa, Y., Takino, T., and Shoji, T., Contribution of slow waves on production of highdensity plasmas by $m=0$ helicon waves, Phys. Plasmas, 6, 4759 (1999). 
Shinohara, S., Propagating wave characteristics for plasma production in plasma processing field, Japanese Journal of Applied Physics, Part 1 (Regular Papers, Short Notes \& Review Papers), 36, 4695 (1997).

Shoji, T., Sakawa, Y., Nakazawa, S., Kadota, K., and Sato, T., Plasma production by helicon waves, Plasma Sources Sci. Technol., 2, 5 (1993).

Stix, T.H., Radiation and Absorption Via Mode Conversion in an Inhomogeneous Collision Free Plasma, Phys. Rev. Lett., 15, 878 (1965).

Swanson, D.G., Plasma Waves, Academic Press, Inc., Boston, 1989.

Wong, K.L., and Ono, M., Parametric decay of lower-hybrid waves in the ACT-1 toroidal device, Phys. Rev. Lett., 47, 842 (1981).

Yun, S., Kim, J.H., and Chang, H.Y., Frequency dependence of helicon wave plasmas near lower hybrid resonance frequency, J. Vac. Sci. Technol. A, 15, 673 (1997).

Yun, S., Suwon, C., Tynan, G., and Hongyoung, C., Density enhancement near lower hybrid resonance layer in $m=0$ helicon wave plasmas, Phys. Plasmas, 8, 358 (2001). 


\section{Chapter 8: Summary of Results}

The ion temperature and wave measurements presented in this dissertation provide compelling evidence for the excitation of slow, "Trivelpiece-Gould", waves and parametrically driven instabilities in helicon sources. As recently noted by Boswell [Boswell, 2001], the roles of slow waves and parametric instabilities in the operation of helicon sources are among the most important issues facing helicon source researchers.

Theoretical studies over the past decade have suggested that coupling to and subsequent damping of the slow wave could explain the large RF absorption efficiencies of helicon source [Cho, 1996; Shamrai and Taranov, 1996; Arnush, 2000]. To this date, no direct measurements of the slow wave have been accomplished. Recent unpublished experimental measurements of large axial currents at the RF frequency in the edge of a helicon source provide strong indirect evidence of a slow wave excitation [Blackwell et al., 2001], however, the wave fields of a slow wave have not been measured in any helicon source. The downstream perpendicular ion temperature measurements presented in this work are consistent with the excitation of slow waves at the edge of the plasma. The perpendicular ion temperature is a maximum when the RF frequency matches the lower hybrid frequency at the plasma edge; only the slow wave has a resonance at the local lower hybrid frequency. The perpendicular ion temperature is largest at the edge of the plasma; only the slow wave propagates at the plasma edge as the density is too low for helicon wave propagation. The ion temperatures are low enough that extremely low phase speed waves are required for direct wave-particle interactions that could result in ion heating; only the perpendicular wavelengths of the slow wave are small enough near the lower hybrid resonance to yield low very low phase speed waves. In fact, the perpendicular wavelengths of the slow wave near resonance are smaller than or equal to the ion mean free path. Thus, ions can exchange momentum with slow waves even though the plasma is highly collisional. The only definitive way to prove the existence of the slow wave would be to directly measure the short wavelength slow wave fluctuations. Laser scattering methods [Wurden, 1982] can measure the large perpendicular wave numbers, $k_{\perp} \geq 100 \mathrm{rad} / \mathrm{cm}$ or $\lambda \leq 1 \mathrm{~mm}$, undetectable with probes. As yet, no such measurements have been made in helicon sources. However, recent microwave scattering 
experiments in a helicon source have detected somewhat longer wavelength fluctuations, $\lambda \gtrsim 1 \mathrm{~mm}$, confirming that wave scattering experiments can be accomplished in helicon sources [Kaganskaya et al., 2001]. Until such measurements are made, these perpendicular ion temperature measurements provide the most conclusive evidence to date of the excitation of slow waves in helicon sources.

The perpendicular ion temperature measurements also provide insight into other aspects of helicon source dynamics. First, in conjunction with previous ion temperature measurements in HELIX [Scime et al., 1998; Balkey et al., 2001], these ion temperature measurements confirm that anomalous ion heating (temperatures in excess of ion-electron thermal equilibration) is characteristic of helicon sources operating at high magnetic field strengths and low RF frequencies regardless of the vacuum chamber geometry. Second, the variation in ion temperature along the source axis demonstrates that the helicon sources plasmas have a small parallel thermal conductivity. Third, the peak in the ion temperature downstream of the antenna confirms that wave energy is directed downstream of the antenna where it then couples RF power directly into the ions. Fourth, the ion heating mechanism downstream of the antenna is in addition to a general background ion heating that, near the RF antenna, is correlated with the appearance of parametrically coupled waves. These four conclusions are based entirely on the experimental data presented in this work.

The experimental measurements of electrostatic and electromagnetic fluctuations presented in this work also yield significant new insights into the physics of helicon plasma sources. First, parametric decay processes provide an important mechanism for dissipation and subsequent conversion of the RF driving wave energy into plasma thermal energy. Second, the parametric decay processes are localized to the center of the plasma column and are correlated with the on-axis lower hybrid frequency. Third, for RF frequencies above above $9 \mathrm{MHz}$ at a magnetic field strength of 845 Gauss, i.e., above the lower hybrid frequency, the parametrically excited waves are purely electrostatic. At the same magnetic field strength, the frequency spectra for an RF frequency of $8 \mathrm{MHz}$ exhibit two distinct sets of parametrically coupled waves, one electrostatic and one electromagnetic. The upper side band of the electrostatic parametrically coupled waves is a backwards propagating wave, i.e. the group velocity and phase velocity were in 
opposite directions. Backwards wave propagation is a characteristic of lower hybrid waves [Swanson, 1989]. Therefore, the upper side band is most likely an electrostatic lower hybrid wave. Fourth, electron temperatures calculated using the ion acoustic wave dispersion relation and the measured frequency and wave number of the parametrically coupled low frequency electrostatic wave are comparable to the electron temperatures measured with a Langmuir probe. Thus, the low frequency electrostatic wave is most likely a parametrically driven ion acoustic wave. Fifth, the perpendicular electromagnetic wave numbers for the pump wave are consistent with $m=+1$ helicon waves and the parallel wave numbers are consistent with the helicon wave dispersion relation as a function of RF frequency. Sixth, differences in the parallel electrostatic and electromagnetic wave numbers for the pump wave suggest that two different waves are propagating at the pump frequency. Seventh, correlations between the perpendicular ion temperatures near the antenna (at low RF driving frequencies and high magnetic field strengths) and the spectral power in the low frequency ion acoustic waves suggests that parametric decay processes might be responsible for perpendicular ion heating near the antenna. Scattering of ions by ion acoustic waves can result in significant levels of ion heating [Stepanov, 1996; Akhiezer et al., 1998]. These seven conclusions are based on the first frequency resolved, combined electrostatic and electromagnetic fluctuation measurements in a helicon plasma source.

Much remains to be learned about how helicon sources operate. Because of the wide range of operational and design parameters, a single theoretical model cannot tell the entire story. However, instead of regarding helicon sources as mysterious "black box," helicon sources can be used as an experimental platform for exploration of important plasma physics issues, i.e., ion heating and parametric decay processes. When helicon sources are used in plasma processing applications, these experiments demonstrate that the operational parameters of the helicon source must be considered carefully in light of the desired plasma parameters. It does matter at what RF frequencies and magnetic field strengths a helicon is operated and ignoring the ion dynamics, both theoretically and experimentally, is unjustified. For example, before the first detailed study of helicon source ion temperatures was completed by Scime in 1998 [Scime et al., 1998], the ion temperatures in helicon sources were routinely assumed to be at room temperature in the 
theoretical models. Since we now know that the ions can reach temperatures of $1.0 \mathrm{eV}$, the ions can contain $25 \%$ of the stored thermal energy in helicon plasmas with electron temperatures of $3 \mathrm{eV}$. Models of RF coupling in helicon sources clearly need to consider the energy content of the ions.

Finally, it is worth noting that these experiments essentially concern the parametric decay of whistler waves in a linear plasma source. In that sense, this research has come full circle. Boswell's original purpose in developing the helicon source was to study space-relevant magnetoplamsas [Boswell and Chen, 1997]. His research in the 1970's focused on whistler wave resonance cones and the parametric decay of whistler waves [Boswell, 1975b; Boswell, 1975a]. From the early 1980's until the mid 1990's the helicon source was primarily considered as plasma processing source [Perry et al., 1991; Chen, 1992; Hershkowitz et al., 1996]. In the late 1990's, the helicon source was reborn as a tool for space-relevant plasma physics experiments [Keiter et al., 2000; Scime et al., 2000] and now parametric decay of whistler waves is once again being studied in compact, linear devices. 


\section{Chapter 8 References}

Akhiezer, A.I., Mikhailenko, V.S., and Stepanov, K.N., Ion-sound parametric turbulence and anomalous electron heating with application to helicon plasma sources, Phys. Lett. A, 245, 117 (1998).

Arnush, D., The role of Trivelpiece-Gould waves in antenna coupling to helicon waves, Phys. Plasmas, 7, 3042 (2000).

Balkey, M.M., Boivin, R.F., Kline, J.L., and Scime, E.E., Ion heating and density production in helicon sources near the lower hybrid frequency, Plasma Sources Sci. Technol., 10, 284 (2001).

Blackwell, B.D., Madziwa, M.D., Arnush, D., and Chen, F.F., Direct Detection of Trivelpiece-Gould Modes in Helicon Discharges, Bulletin of the American Physical Society, 46, 63 (2001).

Boswell, R., Perspective on Current Helicon Source Science Issues, Bulletin of the American Physical Society, 46, 30 (2001).

Boswell, R.W., Measurements of the far-field resonance cone for whistler mode waves in a magnetoplasma, Nature, 258, 58 (1975a).

Boswell, R.W., Non-convective parametric instability associated with whistler wave resonance cone, Phys. Lett. A, 55A, 93 (1975b).

Boswell, R.W., and Chen, F.F., Helicons-the early years, IEEE Trans. Plasma Sci., 25, 1229 (1997).

Chen, F.F., Experiments on helicon plasma sources, J. Vac. Sci. Technol. A, 10, 1389 (1992).

Cho, S., The field and power absorption profiles in helicon plasma resonators, Phys. Plasmas, 3, 4268 (1996).

Hershkowitz, N., Ding, J., Breun, R.A., Chen, R.T.S., Meyer, J., and Quick, A.K., Does high density-low pressure etching depend on the type of plasma source?, Phys. Plasmas, 3, 2197 (1996).

Kaganskaya, N.M., Krämer, M., and Selenin, V.L., Enhanced-scattering experiments on a helicon discharge, Phys. Plasmas, 8, 4694 (2001).

Keiter, P.A., Scime, E.E., Balkey, M.M., Boivin, R., Kline, J.L., and Gary, S.P., Betadependent upper bound on ion temperature anisotropy in a laboratory plasma, Phys. Plasmas, 7, 779 (2000).

Perry, A.J., Vender, D., and Boswell, R.W., The application of the helicon source to plasma processing, Journal of Vacuum Science \& Technology B (Microelectronics Processing and Phenomena), 9, 310 (1991).

Scime, E.E., Keiter, P.A., Balkey, M.M., Bolvin, R.F., Kline, J.L., Blackburn, M., and Gary, S.P., Ion temperature anisotropy limitation in high beta plasmas, Phys. Plasmas, 7, 2157 (2000).

Scime, E.E., Keiter, P.A., Zintl, M.W., Balkey, M.M., Kline, J.L., and Koepke, M.E., Control of ion temperature anisotropy in a helicon plasma, Plasma Sources Sci. Technol., 7, 186 (1998).

Shamrai, K.P., and Taranov, V.B., Volume and surface RF power absorption in a helicon plasma source, Plasma Sources Sci. Technol., 5, 474 (1996). 
Stepanov, K.N., Nonlinear parametric phenomena in plasma during radio frequency heating in the ion cyclotron frequency range, Plasma Phys. Controlled Fusion, 38, A13 (1996).

Swanson, D.G., Plasma Waves, Academic Press, Inc., Boston, 1989.

Wurden, G.A., CO2 Laser Scattering on RF waves in ACT-1, Ph. D. thesis, Princeton University, Princeton (1982). 


\title{
Appendix A
}

\author{
\% This code calculates the perpendicular wave number for a \\ given parallel wave number as a function of magnetic field \\ and radius (for a given density profile). The result shows where \\ the perpendicular wave number is large. In an infinite plasma \\ these conditions would be where the power absorption is greatest. \\ What is lacking is the additional effect of eigenmode resonances \\ where the externally launched wave matches exactly a particular \\ plasma eigenmode. In that situation enhanced absorption can occur \\ because the wave field amplitudes are maximized. Once the k_perp \\ and k_para are obtained using this code, they can be used in the \\ Kline/Spangler code to calculate what the fl would look like. \\ NOTE: Given a typical ion temperate of $0.2 \mathrm{eV}$ and a wave \\ frequency of $9 \mathrm{MHz}$, a k_perp of $800 \mathrm{~cm}-1$ or so would be needed to \\ bring the wave into Landau resonance with the ions. Rotational \\ frame issues will modify this argument. \\ NOTE: At the lower hybrid frequency the sign of k_perp changes \\ for the slow wave and there is no change for the fast wave. Also, \\ the slow wave only has a significant k_perp near the edge of the \\ discharge. Thus, if there is plasma rotation in the edge and the \\ wave fields become large, we could have direct stochastic heating \\ of the flowing ions as they jump around through large electric \\ field. \\ NOTE: Since K-perp increases at smaller radii with increasing \\ magnetic field for the slow wave, the poynting flux should be \\ non-zero deeper into the plasma with increasing magnetic field \\ right up to the lower hybrid transition.

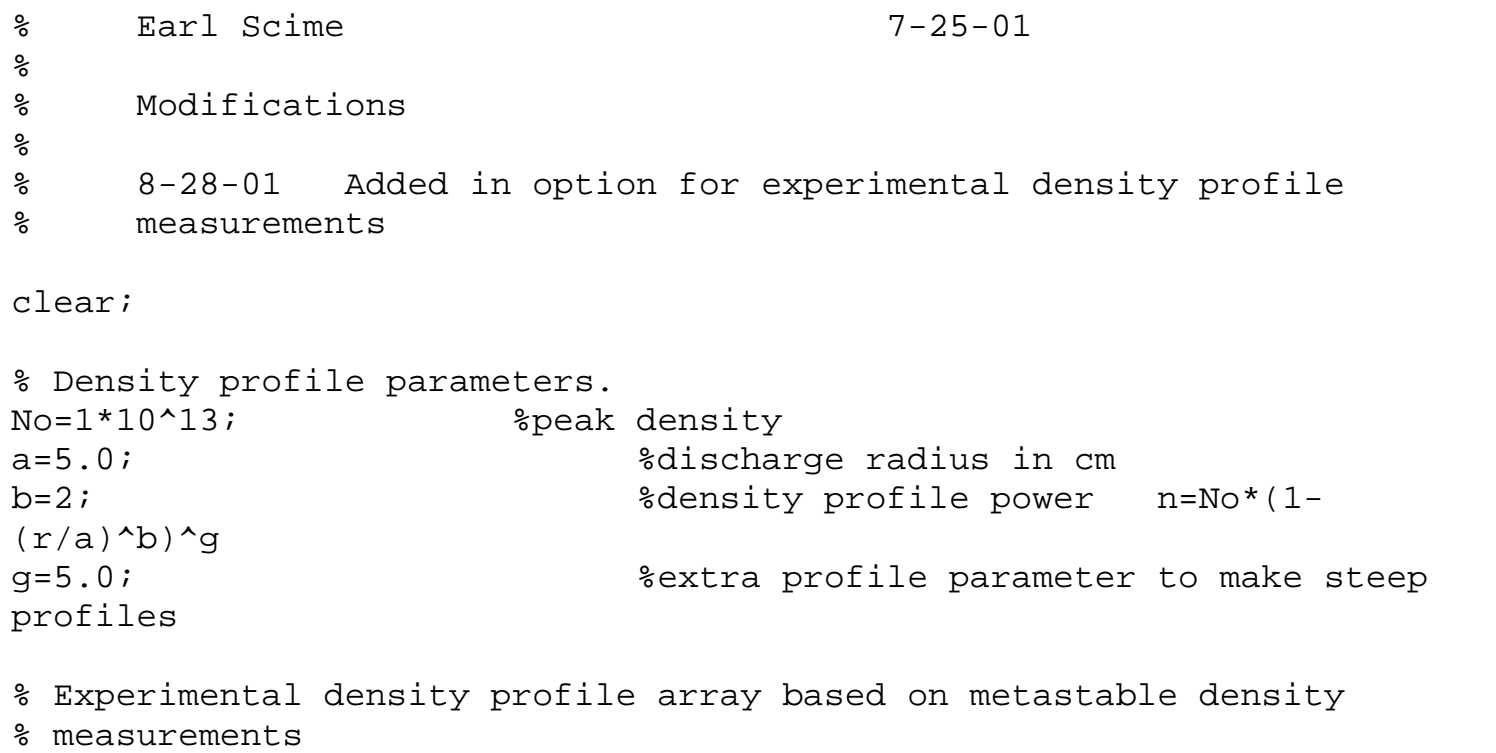


- Note, Ti is pretty constant versus $\mathrm{R}$ so that effect in interpreting \% the area under the curve has been ignored. This data taken at $9 \mathrm{MHz}$ \% so the change as a function of RF frequency are not included. Also, \% PMT voltage was not fixed so profile is more correct than absolute \% value.

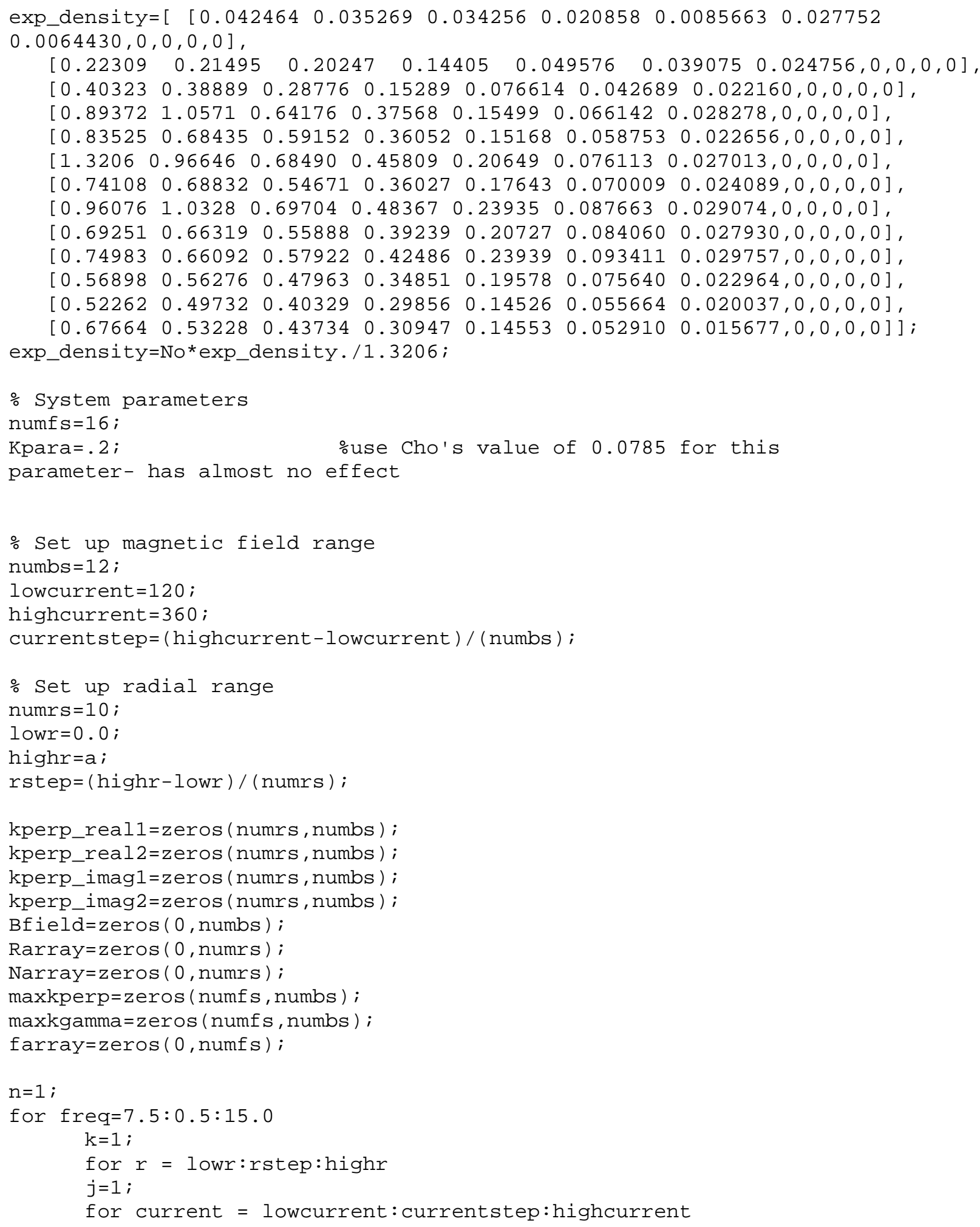




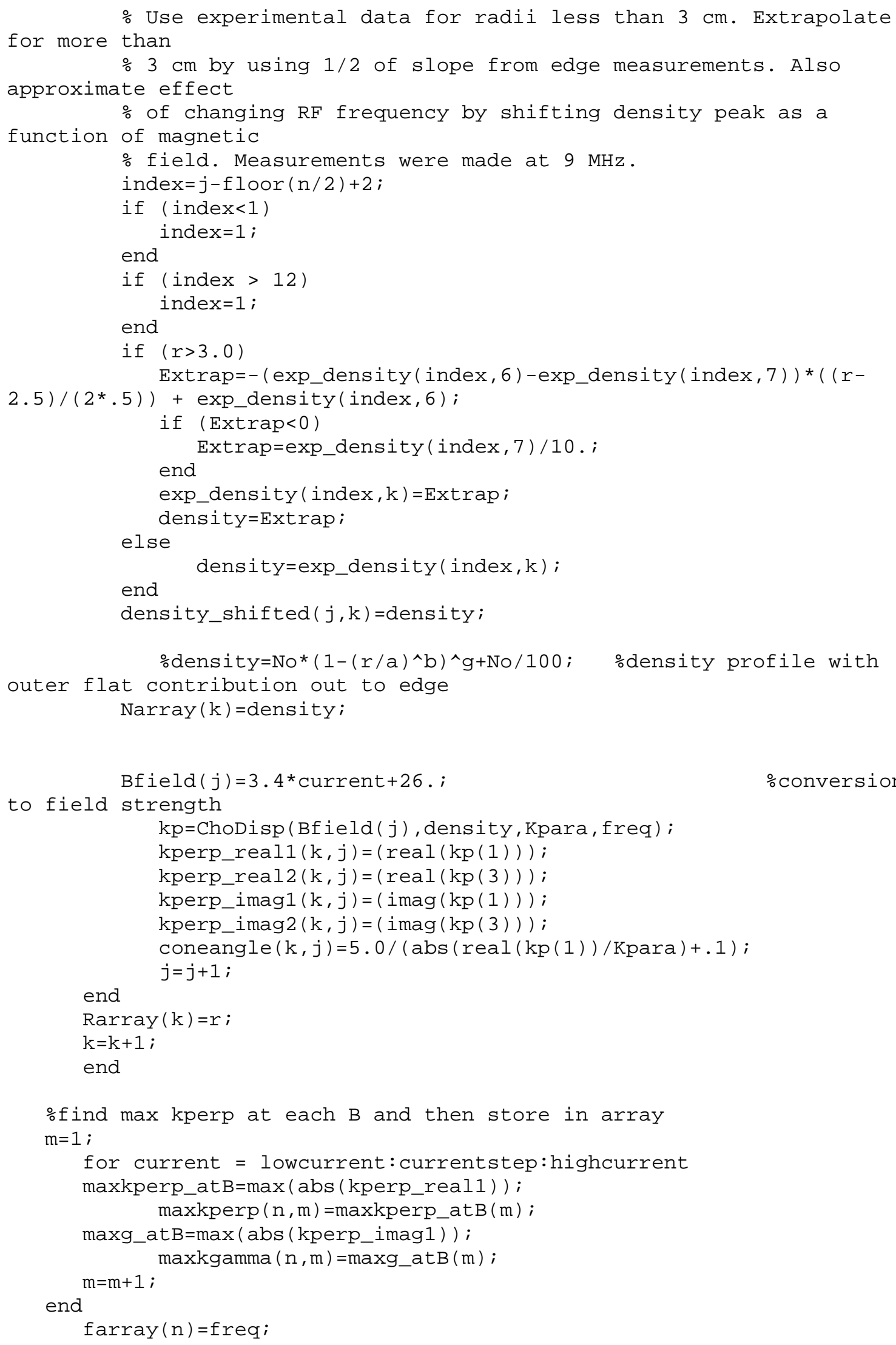




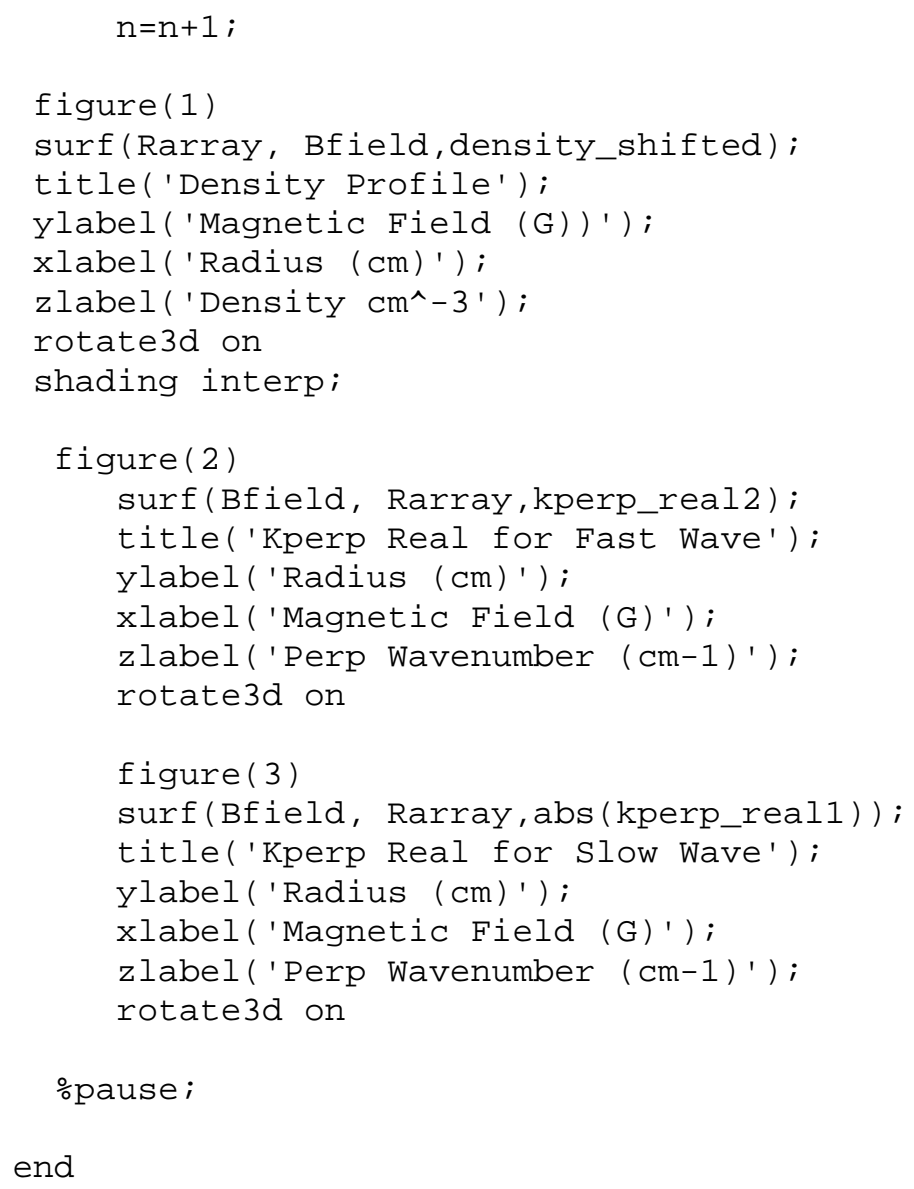




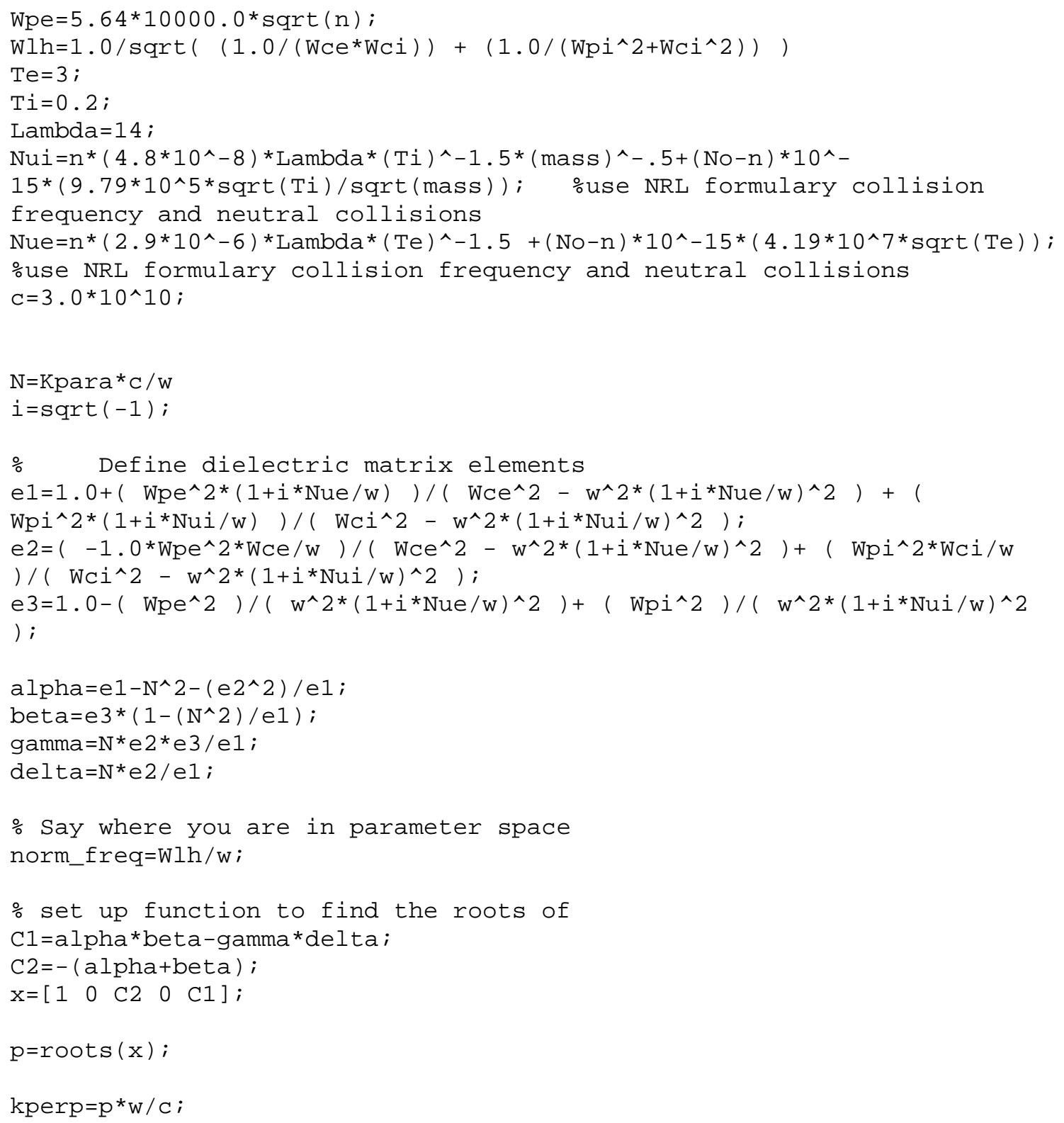




\section{Vitae}

\section{John L. Kline}

\section{Education}

Professional experience

Publications and presentations
Doctor of Philosophy in Physics

2002 West Virginia University

Morgantown, WV

Master of Science in Physics

1998 West Virginia University Morgantown, WV

Bachelor of Science in Physics

1995 University of South Florida Tampa, FL

Graduate Research Assistant

5/1997 - Present West Virginia University Morgantown, WV

Graduate Teaching Assistant

8/1996 - 5/1997 West Virginia University Morgantown, WV

Computer Operator

9/1994-7/1996 The Bradenton Herald Bradenton, FL

Electronic Mechanic

10/1991 - 8/1994 Washington Suburban Sanitary Commission

Laurel, MD

Security Systems Technician

4/1991 - 10/1991 Electronic Services Int. Beltsville, MD

Security Systems Technician

1/1990 - 2/1991 Westec Security Leesburg, VA

Refereed Publications:

J. L. Kline, E. E. Scime, R. B. Boivin, A. M. Keesee, X. Sun, and V. Mikhailenko, 'RF Absorption and Ion Heating in Helicon Sources,' to appear in Phys. Rev. Lett. (2002).

E. Scime, C. Pollock, j.-M. Jahn, J. Kline, and A. Keesee, 'Ion Heating in the Terrestrial Magnetosphere During Substorms and Storm-time: MENA Observations,' to appear in Geophys. Res. Lett. (2002).

E. Scime, J. Kline, D. Mathess, and C. Weber, 'High vacuum rotatable feedthrough,' to appear in Rev. Sci. Instrum. (2002). 
Robert Boivin, John Kline, and Earl Scime, 'Electron Temperature Measurement by a Helium Line Intensity Ratio Method in Helicon plasmas,' Phys. Plasmas, 8, 5303 (2001).

Scott Robertson, Joe Espejo, John Kline, Qudsia Quraishi, Matt Triplett, and Bob Walch, 'Neoclassical effects in the annular Penning Trap,' Phys. Plasmas, 8, 1863 (2001).

Matthew Balkey, Robert Boivin, John Kline, and Earl Scime, 'Ion heating and density production in a helicon source near the lower hybrid frequency,' Plasma Sources Sci. and Tech., 10, 284(2001)

Earl Scime, Robert Boivin, John Kline, and Matthew Balkey, 'Microwave Interferometer for Steady-State Plasmas,' Rev. Sci. Instrum., 72, 1672 (2000)

John Kline, Scott Robertson, Matt Tripplett, Bob Walch, 'Experimental observation of the trapped particle pinch effect,' Physical Review E, 63, 056406-1(2000)

Earl E. Scime, Paul A. Keiter, Matthew M. Balkey, Robert Boivin, John L. Kline, and Melanie Blackburn, 'lon Temperature Anisotropy Limitation in High Beta Plasmas,' Phys. Plasmas, 7, 2157 (2000)

Paul A. Keiter, Earl E. Scime, Matthew M. Balkey, Robert Boivin, John L. Kline and S. Peter Gary 'Beta-Dependent Upper Bound on Ion Temperature Anisotropy in a Laboratory Plasma,' Phys. Plasmas, 7, 779 (2000).

John Kline, Scott Robertson, and Bob Walch, 'An Annular Penning Trap for Studies of Plasma Confinement,' AIP Conference Proceedings, 498, 290 (1999).

J. L. Kline, E. E. Scime, P. A. Keiter, M. M. Balkey and R. F. Boivin, ' Ion Heating in the HELIX Helicon Plasma Source,' Physics of Plasma, 6, 4767 (1999).

Earl E. Scime, Paul A. Keiter, Michael W. Zintl, Matthew M. Balkey, John L. Kline, Mark E. Koepke, 'Control of Ion Temperature Anisotropy in a Helicon Plasma,' Plasma Sources Sci. Technol., 7, 186 (1998).

Invited Talks:

"Ion Heating and Density Production in the HELIX Helicon Source," Los Alamos National Laboratory, Los Alamos, N. M., January 10, 2002.

"Ion Heating and Density Production in the HELIX Helicon Source," Naval Research Laboratory, Washington D. C., November 29, 2001.

"Space Relevant Heat Flux Instabilities Investigated in the Laboratory," 2001 National Radio Science Meeting Boulder, Colorado, January 8, 2001. 
John L. Kline, Earl E. Scime, Matthew M. Balkey, and Robert Boivin, 'Ion Heating and Density Production in the HELIX Helicon Source,' Plasma, Bulletin of the American Physical Society, 46, 131(2001).

John L. Kline, Earl E. Scime, Matthew M. Balkey, Robert Boivin 'Heat Flux Instabilities in Space and Laboratory Plasmas,' Plasma, Bulletin of the American Physical Society, 45, 131(2000).

John L. Kline, Earl E. Scime, Paul A. Keiter, Matthew M. Balkey, Robert Boivin 'Ion Heating in the HELIX Helicon Plasma Source,' Plasma, Bulletin of the American Physical Society, 44, 234(1999).

John L. Kline, Earl E. Scime, Paul A. Keiter, Matthew M. Balkey, Robert Boivin 'Ion Bernstein Wave Heating in a Helicon Plasma, Bulletin of the American Physical Society, 43, 1789(1998).

John L. Kline, Earl E. Scime, Paul A. Keiter, Robert F. Boivin, and Matthew M. Balkey, 'Resonant lon Heating in a Helicon Plasma,' Bulletin of the American Physical Society, 43, 1438, (1998).

John L. Kline, Earl E. Scime, Paul A. Keiter, Matthew M. Balkey, 'Ion Cyclotron Resonant Heating in a Helicon Plasma Source,' IEEE Conference Record - Abstracts, 161(1998).

John L. Kline, Earl E. Scime, Paul A. Keiter, Matthew M. Balkey, 'Ion Cyclotron Resonant Heating in a Helicon Plasma,' Bulletin of the American Physical Society, 42, 1934, (1997).

- More than 30 coauthored poster presentations

\section{Additional professional activities \\ Professional memberships}

Referee: Phys. Plasmas

American Physical Society American Geophysical Union 